

\title{
Computational Chemistry of Compounds with Donor-Acceptor Interactions
}

\section{Victoria \\ UNIVERSITY OF WELLINGTON \\ Te Whare Wānanga \\ o te Ūpoko o te Ika a Māui}

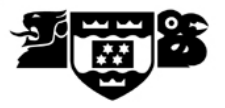

\begin{abstract}
A thesis
submitted to the Victoria University of Wellington in fulfilment of the requirements for the degree of

Doctor of Philosophy

in Chemistry
\end{abstract}

Victoria University of Wellington

(2019) 


\section{Abstract}

Compounds with donor-acceptor interactions find important applications in catalysis, $\mathrm{C}-\mathrm{H}$ activation, phosphorus activation, selective oxidation and cyclization. Moreover, they are potential candidates for use in the synthesis of materials, polymers and lightharvesting systems. The efficient use of a chemical entity is possible when we know its structural and bonding properties. This computational study is intended for the same by studying in detail the structure and bonding properties of donor-acceptor complexes of heavier main-group metals with cyclophane ligands and some heterobimetallic complexes. Additionally, we explored the fluorescence characteristics of benzanthrone dyes.

The first part (i.e. main group metal complexes) involves the exploration of structural features and thermal properties through DFT optimization and then calculating the change in enthalpy of formation for all the possibilities under consideration. For this purpose we selected the last three elements from each of Groups 13, 14 and 15 to explore their different coordination modes with two cyclophane ligands; [2.2.2]paracyclophane and deltaphane. We opted for chlorides of each metal to allow them to coordinate from outside the phenyl rings of the cyclophane cavity and from the top of the cavity. To see the coordination of the metals with the inner core of the selected cyclophanes, we put metal cations in the centre of the cavity and optimized. Subsequently, the bonding properties of these inclusion complexes have been analysed in detail on the basis of Morokuma-Ziegler energy decomposition analysis.

Secondly, we investigated the structure and bonding properties of some indium-zinc heterobimetallic compounds through geometry optimization, NBO analysis and quantum theory of atoms in molecules (QTAIM) analysis-also known as Bader's analysis. We propose that the heterobimetallic reactant involves donor-acceptor bond that cleaves as a result of the addition of mesityl azide. The newly formed complex has In-N and Zn-N bonds.

In the final part benzanthrone dyes containing intramolecular donor-acceptor interactions, (and hence, undergoing intramolecular charge transfer) were subject to the computational investigation of the mechanism of fluorescence taking place in them. Electronic excitations and the structure of first excited state in each case has been discussed thoroughly based on the time-dependent density functional theory. To check for the non-radiative loss of energy, we also performed calculations for the vertical excitations of the triplet states of all the molecules under study. To get a deeper insight into the intramolecular charge transfer, we performed NTO analysis that gives us information based on different colours in regions of charge accumulation and charge depletion. 


\section{Dedicated}

To my best teacher and leader Prophet Muhammad (peace be upon him)

To my teacher Hafiz Muhammad Zahid Sultani

To my parents and grandparents who contributed a lot during my whole study period

To my sisters, maternal uncles and aunts and their families

To my wife and kids

To the whole humanity and those who love it 


\section{Acknowledgements}

All praise and thanks to Allah the Almighty who made me able to reach this stage of my student-carrier to carry out this whole research while enjoying the awe-inspiring beauty of New Zealand and love of the Kiwis.

I am thankful to my supervisor Dr. Matthias Lein and co-supervisor Professor Martyn Coles for all the guidance through which I have been able to complete my research during $\mathrm{PhD}$. They both are a new addition to my list of mentors-for-life. Thanks are also due to Professor John Spencer, Dr. Robin Fulton, Dr. Rob Keyzers and Professor Coles' group for useful discussions. I am grateful to the examiners; Joanne Harvey, Ralf Tonner and Tilo Söhnel too for their time and efforts to give a critical feedback that helped me improve this thesis. Thanks to my senior group-fellows Dr. Muhammad Ali Hashmi and Dr. Julia Schaht for all the guidance and support they have provided. Ali has been more than just a group-fellow; we have been familiar with each other since my MSc and he is always very much caring.

I am grateful to Victoria University of Wellington for awarding me with Victoria Doctoral Scholarship. The orientation workshops, prompt response to the queries and other technical and moral help provided at all the stages especially by Barry Lewis and Patricia Stein is appreciable. Moreover, School of Chemical and Physical Sciences is kind enough to provide the doctoral candidates with work space, computer, stationery, access to the shared facilities such as computing clusters-Heisenberg and Raapoi and opportunities to get experience as tutors and lab demonstrators. Another facility to mention separate is the tea-room with beautiful scenery from the windows that shares work-load of the students by providing them with different drinks. Thanks to Kara Eaton and the rest of administrative staff for all the support you people provided. I am thankful to VUWSA and the organizations under its flagship especially Victoria Clubs to give me a chance to be the part of many co-curricular activities for which I have been awarded Victoria Gold Award 2018. Thanks to the Faculty Strategic Research Grants in addition to my supervisor and the school management who sponsored me for attending a conference at University of Warwick, UK.

The support and prayers which I have been getting through all these years from my parents, grandparents, sisters, wife, maternal uncles and aunts and all the children in the family is unforgettable. Thanks to our elders who have made an environment back at home that never lets me feel alone and far away. It is worth-mentioning that I might have never been able to start my $\mathrm{PhD}$ without the prior support of my family throughout 
my academic career. Even a stage of financial crisis came but they never let me down and kept bearing all the difficulties to pay for my education and other necessities. My mother and paternal grandparents struggled really hard through that period. Also, my father paid for my university studies at a later stage. This was the period when my maternal grandparents, uncles and aunts also kept boosting my courage.

My teachers, previous educational institutes, friends and former work-places contributed to groom my personality. Especially, my mentors Shaikh Hafiz Muhammad Zahid Sultani, Muhammad Aslam khan (Late), Malik Muhammad Akram, Shakeel Ahmed, Rashid Mahmood, Dr. Amjad Ali, Saeed Malik, Qaiser Imtiaz, Zaheer-ud-Din, Professor (Retd.) Gulzar Ahmed Khan, Malik Ghulam Ahmed, Malik Muhammad Sarwar (Late), Malik Sajjad Ahmed, Muhammad Shahpal, Abdur Rehman (Late), Professor Raja Habib ur Rehman (SI), Dr. Khawaja Ansar Yasin, Dr. Tahseen Ghous, Dr. Raja Amjad Waheed, Dr. Zain Ul Abdin, Dr. Muhammad Aziz Chaudhary, Dr. Naeem Ahmed, Dr. Farhan Ahmad Khan, Dr. Aneela Maalik, Dr. Tariq Mehmood, Dr. Khurshid Ayub, Dr. Afsar Khan, Dr. Umar Farooq and Mussarat Hussain Shah played an important role in this regard. I am indebted to Greenland Public Secondary School and Girls Inter College Chowki Kalah for the training and experience that I received there.

I want to extend my gratitude to my friend-like-cousin Muhammad Zahad and friends Muhammad Shoaib Amjad, Raja Rehan Gulzar, Atif Kiani, Afaq Qayyum, Raziq Nawaz, Yasir Bashir, Nasir Riaz, Umar Khan, Tayyab Bashir, Attiq-ur-Rehman, Mohammed Nofal, Abubakar Siddique, Hassan Tariq, Mohammed Alshaboti, Baligh Alhilali and Sami Aljohani for all their time, company and moral support. Special thanks to Muhammad Shabbir Abbasi and Junaid Haseeb for their every kind of support especially during the final year of my PhD. I want to mention hundreds of my students who continue to express their well wishes; thanks to all of them.

To summarize, there might be still many names left out (including the rest of my family and friends) who have increased my strength and courage through their prayers and well wishes to reach here. I want to extend my gratitude to all such people. 


\section{Contents}

$\begin{array}{ll}\text { Abstract } & \text { ii }\end{array}$

$\begin{array}{lll}\text { Dedications } & \text { iii }\end{array}$

Acknowledgements

Table of Contents vi

List of Figures $\quad x$

$\begin{array}{ll}\text { List of Schemes } & \text { Xv }\end{array}$

$\begin{array}{ll}\text { List of Tables } & \text { xvii }\end{array}$

$\begin{array}{lll}\text { Glossary } & \text { xxvi }\end{array}$

1 Introduction 1

1.1 Heavier Main-Group Metal Complexes . . . . . . . . . . . . . . 2

1.1.1 Transition Metal-like Behaviour . . . . . . . . . . . . . 3

1.1.1.1 Group 13 Compounds . . . . . . . . . . . . 4

1.1.1.2 Group 14 Complexes ............. 6

1.1.1.3 Group 15 Complexes . . . . . . . . . . 8

1.1.2 Metal-Cyclophane Host-Guest Complexes . . . . . . . . . . . . 11 
1.1.3 Bonding Properties . . . . . . . . . . . . . . 18

1.1.4 Reactivity ................... 20

1.2 Benzanthrone-Based Fluorescent Dyes . . . . . . . . . . . . . 23

1.3 Aim of the Thesis . . . . . . . . . . . . . . . 28

2 Computational Details $\quad 30$

2.1 Basic Concepts of Density Functional Theory . . . . . . . . . . . . . . 32

2.1.1 DFT Methods Dealing with Exchange-Correlation Energy . . . 34

2.1.1.1 Local density approximation (LDA) . . . . . . 35

2.1.1.2 Generalized Gradient Approximation (GGA) . . . . . 35

2.1.1.3 Meta-GGA and Hyper-GGA Methods . . . . . . 36

2.1 .2 Basis Sets . . . . . . . . . . . . . . . . 37

2.1.2.1 Types of Basis Sets . . . . . . . . . . . 39

2.1.2.2 Commonly Used Basis Sets . . . . . . . . . . . . 40

2.2 Size of a System and Accuracy . . . . . . . . . . . . . . . . 41

2.3 Methodology in the Current Study . . . . . . . . . . . . . . . . 41

2.3.1 Geometry Optimization . . . . . . . . . . . . 42

2.3.2 Morokuma-Ziegler Energy Decomposition Analysis (MZEDA) 43

2.3.3 Excited States . . . . . . . . . . . . . . . . . . . . 44

2.3.4 Technical Details of Our Methodology . . . . . . . . . . . 46

3 Coordination of Cyclophanes with Heavier Main-Group Metals 47

3.1 Metal-[2.2.2]paracyclophane Complexes . . . . . . . . . . . . . 49

3.1 .1 Structural Features $\ldots \ldots . \ldots . \ldots 49$

3.1.1.1 [2.2.2]paracyclophane $(p \mathrm{Cp}) \ldots \ldots . \ldots$

3.1.1.2 Exclusion Complexes . . . . . . . . . . . 52 
3.1.1.3 Inclusion Complexes _............ 61

3.1.2 Thermodynamic Parameters .............. 71

3.1.3 MZEDA of the $p \mathrm{Cp}-\mathrm{M}^{\mathrm{n}+}$ Complexes $\ldots \ldots \ldots \ldots 74$

3.2 Metal-Deltaphane Complexes . . . . . . . . . . . . . . . 78

3.2.1 Structural Properties . . . . . . . . . . . . 78

3.2.1.1 Free Deltaphane . . . . . . . . . . . 78

3.2.1.2 Exclusion Complexes . . . . . . . . . . . 79

3.2.1.3 Inclusion Complexes . . . . . . . . . . . 84

3.2.2 Thermodynamic Properties . . . . . . . . . . . . . 89

3.2.3 MZEDA of the Dp- $\mathrm{M}^{\mathrm{n}+}$ Complexes . . . . . . . . . . . 90

3.3 Comparison of $p \mathrm{Cp}$ and $\mathrm{Dp}$ Complexes $\ldots \ldots$. . . . . . . . . 93

3.4 Conclusion . . . . . . . . . . . . . . . . . . 94

4 A Computational Study of Structure and Bonding Properties of a Heterobimetallic Indium-Zinc Compound with Mesityl Azide 96

4.1 Investigation of Structural and Bonding Properties of Heterobimetallic Compounds: Indium-Zinc bond cleavage . . . . . . . . . . . . 97

$4.1 .1 \quad$ Structural Features . . . . . . . . . . . . . . 97

4.1.2 Bonding Properties . . . . . . . . . . . . . . . 98

4.1 .3 Conclusion ...................... 101

5 Fluorescence Characteristics of Aminobenzanthrone Dyes 102

5.1 Structural Properties and Energetics of the Ground-state Structures . . . 104

5.1.1 Stability of $\mathrm{C}=\mathrm{O}$ in Benzanthrone Framework . . . . . . . . . 106

5.1 .2 NBO Analysis . . . . . . . . . . . . . 107

5.1 .3 FMO Analysis . . . . . . . . . . . . . . 108

5.2 Excited States . . . . . . . . . . . . . . . . . . . . 109 
5.2.1 Vertical Excitations . . . . . . . . . . . . . . 111

5.2.1.1 NTO Analysis . . . . . . . . . . . . 113

5.2.2 Excited State Optimization . . . . . . . . . . . . . . 115

5.2.3 Comparison of Solvated Ground State and Excited State Structures . . . . . . . . . . . . . . . 117

5.2.4 Triplet State Vertical Excitations . . . . . . . . . . . . . 118

5.3 Conclusion . . . . . . . . . . . . . . . . . . 119

6 Summary and Outlook 122

$\begin{array}{lr}\text { Bibliography } & 125\end{array}$ 


\section{List of Figures}

1.1 A stable digermavinylidene . . . . . . . . . . . . . . 7

1.2 Lewis acid-base type complexes of Group 15 metals with that of Group 13 in which M15 acts as donor and M13 behaves as acceptor . . . . . 9

1.3 Cyclophane ligands of prism-shaped cavity capable of hosting electrondeficient metals. These are also the ligands of our interest in the study of coordination of main-group cyclophane complexes. $p C p$ represents [2.2.2] paracyclophane and $\mathrm{Dp}$ denotes deltaphane. . . . . . . . . 11

1.4 Crystal structure of $p$ Cp. $\mathrm{C}_{6} \mathrm{~N}_{4}\left(\mathrm{CSD} 1129300^{114}\right) \ldots \ldots \ldots$

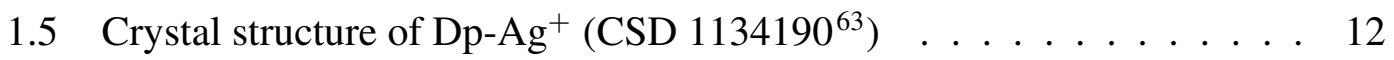

1.6 Results of some pioneering studies regarding cyclophane complexes with main-group metals . . . . . . . . . . . . . . . 13

1.7 Crystal structure of $p \mathrm{Cp}-\mathrm{Ga} \cdot \mathrm{GaBr}_{4}\left(\mathrm{CSD} 1156091^{116}\right) \ldots \ldots \ldots$

1.8 Crystal structure of $p \mathrm{Cp}-\mathrm{GeCl}^{+} .\left(\mathrm{Al}_{4} \mathrm{Cl}_{10} \mathrm{O}_{2}\right)^{2-} .2 \mathrm{C}_{6} \mathrm{H}_{6}\left(\mathrm{CSD} 1196799^{117}\right) 14$

1.9 Crystal structure of $p \mathrm{Cp}-\mathrm{Sn} .2 \mathrm{AlCl}_{4} \cdot 2 \mathrm{C}_{6} \mathrm{H}_{6}\left(\mathrm{CSD} 1196798^{117}\right) \ldots \ldots$

1.10 Crystal structure of (20) $p \mathrm{Cp}_{\mathrm{As}}\left(\mathrm{CSD} 1197586^{110}\right) \ldots \ldots \ldots$

1.11 Crystal structure of (21) $p \mathrm{Cp} 2_{\mathrm{Sb}}\left(\mathrm{CSD} 1197587^{110}\right) \ldots \ldots \ldots$

1.12 Crystal structure of (22) $p \mathrm{Cp}_{\mathrm{Bi}}\left(\mathrm{CSD} 1197588^{110}\right) \ldots \ldots \ldots$

1.13 Trigonal bipyramidal coordination of $\mathrm{Ga}^{+} \ldots \ldots \ldots$. . . . . . . 17

1.14 Canonical forms for alkyne analogues where $\mathrm{E}=\mathrm{Ge}, \mathrm{Sn}$ and $\mathrm{Pb} \ldots 19$

1.15 Heterobimetllic complexes of $\mathrm{Ga}$ and In containing Ni-M interactions (where $\mathrm{M}=\mathrm{Ga}$ and $\mathrm{In}) \ldots \ldots \ldots 21$

1.16 Benzanthrone ......................... 24 
1.17 Crystal structure of a 3-aminobenzanthrone dye (38) reported by Kirilova et al. ${ }^{166}$ Hydrogen atoms omitted for clarity. Reprinted with permission under the copyright licence number: 4694951097894 from the publisher. Copyright (2009) Elsevier B.V. . . . . . . . . . . . . . . 27

1.18 Synthesis of an enol at $\mathrm{C}=\mathrm{O}$ in benzanthrone framework along with the substitution of a diphenyl at postion- 6 of benzanthrone framework. . . .

2.1 Mean absolute deviation (MAD) of different functionals from the reference CCSD(T) in the benchmark study by Grimme et al. ${ }^{193}$ Copyright (2013) Marc Steinmetz and Stefan Grimme. ChemistryOpen published by WILEY-VCH Verlag GmbH \& Co. KGaA, Weinheim. . . . . . . . . 37

2.2 Mechanism of a molecule going to the first excited state from ground state ......................... . . . . . 45

3.1 Structures of exclusion complexes: $p \mathrm{Cp}\left(\mathrm{MCl}_{\mathrm{n}}\right)_{3}\left(p \mathrm{Cp} 3_{\mathrm{M}}\right), p \mathrm{Cp}\left(\mathrm{MCl}_{\mathrm{n}}\right)_{2}$ $\left(p \mathrm{Cp} 2_{\mathrm{M}}\right), \mathrm{Dp}\left(\mathrm{MCl}_{\mathrm{n}}\right)_{3}\left(\mathrm{Dp} 3_{\mathrm{M}}\right), \mathrm{Dp}\left(\mathrm{MCl}_{\mathrm{n}}\right)_{2}\left(\mathrm{Dp} 2_{\mathrm{M}}\right)$ and inclusion complexes: $p \mathrm{CpMCl}_{\mathrm{n}}\left(p \mathrm{Cp} 1_{\mathrm{M}}\right), \mathrm{DpMCl}_{\mathrm{n}}\left(\mathrm{Dp} 1_{\mathrm{M}}\right), p \mathrm{Cp}-\mathrm{M}^{\mathrm{n}+}$ and $\mathrm{Dp}-\mathrm{M}^{\mathrm{n}+}$. In all the exclusion complexes $p \mathrm{Cp} 3_{\mathrm{M}}, p \mathrm{Cp} 2_{\mathrm{M}}, \mathrm{Dp} 3_{\mathrm{M}}$ and $\mathrm{Dp} 2_{\mathrm{M}}$ metal chlorides approach the cyclophane cavity from the outer surfaces of the aryl rings. Metal chlorides in inclusion complexes $\left(p \mathrm{Cp} 1_{\mathrm{M}}\right)$ and $\left(\mathrm{Dp} 1_{\mathrm{M}}\right)$ coordinate with the top of the cavity. Metal ions $\left(\mathrm{M}^{\mathrm{n}+}\right)$ in inclusion complexes $p \mathrm{Cp}-\mathrm{M}^{\mathrm{n}+}$ and $\mathrm{Dp}-\mathrm{M}^{\mathrm{n}+}$ are incorporated inside the cyclophane cavity. . . . . . . . . . . . . . . 50

3.2 Structure of $\mathrm{D}_{3}$ symmetric $p$ Cp optimized at PBE0-D3/def2TZVP level of theory. All the atom numbering corresponds to the numbering used in discussion. Hydrogen atoms are omitted for clarity. . . . . . . . . . .

3.3 Structures of exclusion complexes of [2.2.2]paracyclophane ( $p \mathrm{Cp}$ ) with metal chlorides forming $p \mathrm{Cp}\left(\mathrm{MCl}_{n}\right)_{3}\left(p \mathrm{Cp} 3_{\mathrm{M}}\right)$ optimized at PBE0-D3BJ/def2TZVP level. 'M' represents Ga (for Goup 13), Ge (for Group 14) and As (for Group 15). Metal chlorides in these complexes coordinate with the outer surfaces of phenyl rings of $p \mathrm{Cp}$. It is to be noted that metal-carbon bonds have been drawn to indicate distances. For clarity, attachment of metal is shown with different carbon atoms on each phenyl ring to observe the overall position and the mode of interaction of the metal chloride. Some atom labels have been magnified as they are used in discussion in conjunction with Figure $3.2 . \ldots . . . . . .53$ 
3.4 Structures of exclusion complexes of [2.2.2]paracyclophane ( $p \mathrm{Cp})$ with metal chlorides forming $p \mathrm{Cp}\left(\mathrm{MCl}_{n}\right)_{2}\left(p \mathrm{Cp} 2_{\mathrm{M}}\right)$ optimized at PBE0-D3BJ/def2TZVP level. 'M' represents Ga (for Goup 13), Ge (for Group 14) and As (for Group 15). Metal chlorides in these complexes coordinate with the outer surfaces of phenyl rings of $p \mathrm{Cp}$. It is to be noted that metal-carbon bonds have been drawn to indicate distances. For clarity, attachment of metal is shown with different carbon atoms on each phenyl ring to observe the overall range of metal-carbon distances and the mode of interaction of the metal chloride.

3.5 Structure of $p \mathrm{Cp} 2_{\mathrm{Sb}}$ optimized at PBE0-D3/def2TZVP level of theory showing the range of metal-carbon distances on each phenyl ring. . . . 58

3.6 Structure of $\mathrm{C}_{3}$ symmetric $p \mathrm{Cp} 3_{\mathrm{Bi}}$ optimized at PBE0-D3/def2TZVP level of theory showing the range of metal-carbon distances on each phenyl ring. . . . . . . . . . . . . . .

3.7 Structures of the inclusion complexes of [2.2.2]paracyclophane ( $p \mathrm{Cp})$ with metal chlorides forming $p \mathrm{Cp}\left(\mathrm{MCl}_{\mathrm{n}}\right)$ denoted as $p \mathrm{Cp} 1_{\mathrm{M}}$ optimized at PBE0-D3BJ/def2TZVP level. "M" represents $\mathrm{Ga}$, In and Tl, Ge (for Group 14) and As (for Group 15). In $p \mathrm{Cp}_{\mathrm{M}}$, metal chlorides coordinate with the top of the cavity. It is to be noted that metal-carbon bonds have been drawn to indicate distances with the top two carbon atoms of each phenyl ring. All distances in $\AA$. . . . . . . . . . . . . . . . . . . .

3.8 Structures of the inclusion complexes of [2.2.2]paracyclophane ( $p \mathrm{Cp})$ with metal cations embedded in the cavity $\left(p \mathrm{Cp}-\mathrm{M}^{\mathrm{n}+}\right)$ optimized at PBE0-D3BJ/def2TZVP level. "M" represents Ga (for Group 13), Ge (for Group 14) and As (for Group 15). The $p \mathrm{Cp}-\mathrm{M}^{\mathrm{n}+}$ complexes have identical metal-carbon distances with each phenyl ring. The $p \mathrm{Cp}-\mathrm{M}^{+}$ complexes of Group 13 and $p \mathrm{Cp}-\mathrm{As}^{3+}$ are $\mathrm{C}_{3}$ symmetric in their ground state. Group 14 and all other Group $15 p \mathrm{Cp}-\mathrm{M}^{\mathrm{n}+}$ are $\mathrm{D}_{3}$ symmetric in their ground state . . . . . . . . . . . . . . . . .

3.9 Structures of $\mathrm{D}_{3}$ symmetric Dp optimized at PBE0-D3/def2TZVP level of theory. All the atom numbering corresponds to the numbering used in discussion. Hydrogen atoms are omitted for clarity. . . . . . . . . . .

3.10 Structures of exclusion complexes of deltaphane (Dp) with metal chlorides forming $\mathrm{Dp}\left(\mathrm{MCl}_{\mathrm{n}}\right)_{3}\left(\mathrm{Dp} 3_{\mathrm{M}}\right)$. "M" represents $\mathrm{Ga}$ (for Goup 13), Ge (for Group 14) and As (for Group 15). Metal chlorides in these complexes coordinate with the outer surfaces of phenyl rings of Dp. It is to be noted that metal-carbon bonds have been drawn to indicate distances. For clarity, attachment of metal is shown with different carbon atoms on each phenyl ring to observe the overall position and the mode of interaction of the metal chloride. . . . . . . . . . . . . 81 
3.11 Structures of exclusion complexes of deltaphane (Dp) with metal chlorides forming $\mathrm{Dp}\left(\mathrm{MCl}_{\mathrm{n}}\right)_{2}\left(\mathrm{Dp} 2_{\mathrm{M}}\right)$. "M" represents $\mathrm{Ga}$ (for Goup 13), Ge (for Group 14) and As (for Group 15). Metal chlorides in these complexes coordinate with the outer surfaces of phenyl rings of Dp. It is to be noted that metal-carbon bonds have been drawn to indicate distances. For clarity, attachment of metal is shown with different carbon atoms on each phenyl ring to observe the overall position and the mode of interaction of the metal chloride. . . . . . . . . . . . . . . 82

3.12 Structures of the inclusion complexes of deltaphane (Dp) with metal chlorides forming $\mathrm{Dp}\left(\mathrm{MCl}_{\mathrm{n}}\right)$ denoted as $\mathrm{Dp} 1_{\mathrm{M}}$ optimized at PBE0-D3BJ/def2TZVP level. $M$ represents Ga (for Group 13), Ge (for Group 14) and As (for Group 15). In Dp1 $1_{\mathrm{M}}$, metal chlorides coordinate with the outer peripheries of the phenyl rings of $\mathrm{Dp}$ from above the cavity. It is to be noted that metal-carbon bonds have been drawn to indicate distances.

3.13 Structures of the inclusion complexes of deltaphane (Dp) with metal cations embedded in the cavity $\left(\mathrm{Dp}-\mathrm{M}^{\mathrm{n}+}\right)$ optimized at PBE0-D3BJ/def2TZVP level. $M$ represents Ga (for Group 13), Ge (for Group 14) and As (for Group 15). . . . . . . . . . . . . . . . . . 88

4.1 Ground state structures of the main reactant, intermediate and product in Scheme 4.1 optimized at PBE0-D3BJ/def2SVP level. Some important bond lengths including those on the reaction site have also been indicated.

5.1 Proposed mechanism of intramolecular charge transfer in benzanthrone

5.2 Experimentally known fluorescence spectra of the dyes under current study. ${ }^{172}$ Reprinted with permission from the copyright owner under licence number: 4694530175556. Copyright (2012) Springer Science Business Media, LLC.

5.3 Selected 3-aminobenzanthrone dyes involved in the current study . . . 105

5.4 Elaboration of charge transfer in ethanol phase from occupied to unoccupied orbitals as observed in selected benzanthrone dyes through second order perturbation theory (employed in NBO analysis). Since the mechanism is same (with small difference in the intensity of each transfer) in benzanthrone framework of all the molecules, only the substituents are given for $\mathbf{2}_{\mathrm{AB}}, \mathbf{3}_{\mathrm{AB}}$ and $\mathbf{4}_{\mathrm{AB}}$. Varying colours are intended for clarity. All energies are in $\mathrm{kcal} / \mathrm{mol}$. . . . . . . . . . . . 108

5.5 HOMOs and LUMOs of the gas phase benzanthrone dyes under discussion . . . . . . . . . . . . . . . . . 110 
5.6 Flow of charge density from HOTO to LUTO of the molecules visualized as a result of NTO analysis. Dark-blue colour indicates HOTO (charge depletion) while Light-blue is for LUTO (charge accumulation) . $\mathbf{N}_{\mathbf{A B}}$ and $\mathbf{N}_{\mathbf{A B} \text { (ethanol) }}$ represent gas phase and ethanol phase structures of the NTOs respectively where $N$ indicates $1,2,3$ and 4 for the corresponding structure. . . . . . . . . . . . . . . . . 114 


\section{List of Schemes}

1.1 Sandwich complexes of Group 13 lower $\mathrm{M}^{+}$cations with TEF. Reprinted with permission from reference ${ }^{66}$. Copyright (1999) American Chemical Society. . . . . . . . . . . . . . . . 5 5

1.2 Synthesis of Group 13 formazanido complexes. Reprinted with permission from reference ${ }^{69}$ - Published by The Royal Society of Chemistry under Creative Commons Attribution-NonCommercial 3.0 Unported Licence. . . . . . . . . . . . . . . . . 6

1.3 Synthesis of $\mathrm{GeH}_{2}$ and $\mathrm{SnH}_{2}$ analogues. NHC moiety acts as a donor liginad while pentacarbonyl tungsten $(0)$ is acceptor ligand. Reprinted with permission from reference ${ }^{89}$. Copyright (2010) American Chemical Society. . . . . . . . . . . . . . . . . . 8

1.4 Synthesis of a $\mathrm{Au} \rightarrow \mathrm{Bi}$ complex. Reprinted with permission (Licence number: 4694270339676) from reference ${ }^{139}$ Copyright (2012) John Wiley and Sons. . . . . . . . . . . . . . . . . . 21

1.5 Coordination of a chlorobismuthine with a phenylphosphinopalladium chloride. Reprinted with permission (Licence number: 44694270721888) from reference ${ }^{139}$ Copyright (2012) John Wiley and Sons . . . . . . . . 22

1.6 Heterobimetallic compounds with Ga-M bonds. Reprinted with permission (Licence number: 1000314-1) from reference ${ }^{141}$. Copyright (2012) Royal Society of Chemistry. . . . . . . . . . . . . . . . . . . . 23

1.7 Fluorescent amidobenzanthrone dyes. Reprinted with permission (Licence number: 4702151245259) from reference ${ }^{149}$. Copyright (2012) Elsevier B. V.

1.8 Mechanism of electropolymerization of benzanthrone. Reprinted with permission from reference ${ }^{162}$. Copyright (2009) American Chemical Society. . . . . . . . . . . . . . . . . . 26 
4.1 Reaction involving the indium zinc bond in InZn-1 to add mesityl azide which results in intermediate (Int-InZn) containing 4-membered cyclic and subsequent elimination of $\mathrm{N}_{2}$ to form InZn-2. Hydrogen atoms are omitted for clarity. Reprinted from reference ${ }^{217}$ with permission (Licence ID: 1001280-1) from the publisher. Copyright (2019) Royal Society of Chemistry. . . . . . . . . . . . . . . . . . . . . . 98 


\section{List of Tables}

3.1 Selected experimental bond lengths and dihedral angles vs their corresponding calculated values in [2.2.2]paracyclophane $(p \mathrm{Cp})$. ' $\mathrm{r}$ ' is for bond length $(\AA)$ and $\phi$ reflects a dihedral angle (degrees). It is to be noted that the optimized $\mathrm{D}_{3}$-symmetric geometry has the three bridging bond lengths identical, all the six carbon bridgeheads equivalent, equal $\mathrm{C}-\mathrm{C}$ bond lengths in the aryl rings and the alternative repetition of dihedral angles on all the six bridgeheads. Hence, all these values are written once and not repeated. The experimental data from the crystal structure of $p C p \cdot C_{6} \mathrm{~N}_{4}$ is given in square brackets. . . . . . . . . . . . 51

3.2 Metal-carbon distances in $p \mathrm{Cp}$-gallium exclusion complexes. Bold text indicates the presence of coordination based on the sum of van der Waal $\operatorname{radii}^{213}$ that is $\mathrm{Ga}(1.87)+\mathrm{C}(1.70)=3.57 \AA . \ldots 54$

3.3 Metal-carbon distances in $p \mathrm{Cp}$-indium exclusion complexes. Bold text indicates the presence of coordination based on the sum of van der Waal radii that is $\operatorname{In}\left(1.98^{214}\right)+\mathrm{C}\left(1.70^{215}\right)=3.68 \AA . \ldots \ldots 5$

3.4 Metal-carbon distances in $p \mathrm{Cp}$-thalium exclusion complexes. Bold text indicates the presence of coordination based on the sum of van der Waal radii that is $\mathrm{Tl}\left(2.08^{216}\right)+\mathrm{C}\left(1.70^{215}\right)=3.78 \AA . . . . . . . . .555$

3.5 Metal-carbon distances in $p \mathrm{Cp}$-germanium exclusion complexes. Bold text indicates the presence of coordination. The sum of van der Waal radii that is $\mathrm{Ge}\left(2.11^{216}\right)+\mathrm{C}\left(1.70^{215}\right)=3.81 \AA$ shows that all the M-C distances are classified as coordination but the visual representation of the complexes shows otherwise. Hence, the classification of coordination is based on three-dimensional view. . . . . . . . . . . . . .

3.6 Metal-carbon distances in $p \mathrm{Cp}$-tin exclusion complexes. Based on the sum of van der Waal radii that is $\operatorname{Sn}\left(2.17^{213}\right)+C\left(1.70^{215}\right)=3.87$ $\AA$ shows that all the M-C distances are classified as coordination but the visual representation of the complexes shows otherwise. Hence, the classification of coordination is based on three-dimensional view. . . . . 56 
3.7 Metal-carbon distances in $p$ Cp-lead exclusion complexes. Based on the sum of van der Waal radii that is $\mathrm{Pb}\left(2.02^{213}\right)+\mathrm{C}\left(1.70^{215}\right)=3.72 \AA$, shows that all the M-C distances are classified as coordination but the visual representation of the complexes shows otherwise. Hence, the classification of coordination is based on three-dimensional view. . . . .

3.8 Metal-carbon distances in $p \mathrm{Cp}$-arsenic exclusion complexes. Bold text indicates the presence of coordination based on the sum of van der Waal radii that is As $\left(1.85^{213}\right)+\mathrm{C}\left(1.70^{215}\right)=3.55 \AA$. . . . . . . . . . . 56

3.9 Metal-carbon distances in $p \mathrm{Cp}$-antimony exclusion complexes. Bold text indicates the presence of coordination based on the sum of van der Waal radii that is $\mathrm{Sb}\left(2.06^{213}\right)+\mathrm{C}\left(1.70^{215}\right)=3.76 \AA$. $p \mathrm{Cp} 2{ }_{\mathrm{Sb}} \cdot \mathrm{C}_{6} \mathrm{H}_{6}$ is known experimentally ${ }^{110}$ and its values are given in parentheses for comparison. . . . . . . . . . . . . . . . 58

3.10 Selected experimental bond lengths and dihedral angles vs their corresponding calculated values in $p \mathrm{Cp} 2_{\mathrm{Sb}}$. ' $\mathrm{r}$ ' is for bond length $(\AA)$ and $\phi$ reflects a dihedral angle (degrees). . . . . . . . . . . 58

3.11 Metal-carbon distances in $p$ Cp-bismuth complexes. Bold text indicates the presence of coordination based on the sum of van der Waal radii that is $\mathrm{Bi}\left(2.07^{213}\right)+\mathrm{C}\left(1.70^{215}\right)=3.77 \AA . p \mathrm{Cp} 3_{\mathrm{Bi}}$ is known experimentally, therefore its structural data has also been incorporated in parentheses.

3.12 Selected experimental bond lengths and dihedral angles vs their corresponding calculated values in $p \mathrm{Cp} 3_{\mathrm{Bi}}$. ' $\mathrm{r}$ ' is for bond length $(\AA)$ and $\phi$ reflects a dihedral angle (degrees). . . . . . . . . . . . .

3.13 Metal-carbon distances in $p \mathrm{Cp}$-gallium complexes. In $p \mathrm{Cp} 1_{\mathrm{Ga}}$, the $\mathrm{M}-\mathrm{C}$ bond distances are shown for two top of the cavity carbon atoms of each of the three arene rings. $\mathrm{Ga}^{+}$in $p \mathrm{Cp}-\mathrm{Ga}^{+}$has similar interaction with the three phenyl rings, so bond distances written just for one phenyl ring. Bold text indicates the presence of coordination based on the sum of van der Waal radii $^{213}$ that is $\mathrm{Ga}(1.87)+\mathrm{C}(1.70)=3.57 \AA . . . .$. .

3.14 Selected experimental bond lengths and dihedral angles vs their corresponding calculated values in $p \mathrm{Cp}-\mathrm{Ga}^{+}$. ' $\mathrm{r}$ ' is for bond length $(\AA)$ and $\phi$ reflects a dihedral angle (degrees) . . . . . . . . . . . . . .

3.15 Metal-carbon distances in $p \mathrm{Cp}$-indium complexes. In $p \mathrm{Cp} 1_{\mathrm{In}}$, the $\mathrm{M}$ $\mathrm{C}$ bond distances are shown for two top of the cavity carbon atoms of each of the three arene rings. $\mathrm{In}^{+}$has similar interaction with the three phenyl rings, so bond distances written for only one phenyl ring. Bold text indicates the presence of coordination based on the sum of van der Waal radii that is $\operatorname{In}\left(1.98^{214}\right)+C\left(1.70^{215}\right)=3.68 \AA . . . . . . .664$ 
3.16 Metal-carbon distances in $p \mathrm{Cp}$-thalium complexes inclusion complexes. In $p C p 1_{\mathrm{Tl}}$, the $\mathrm{M}-\mathrm{C}$ bond distances are shown for two top of the cavity carbon atoms of each of the three arene rings. $\mathrm{Tl}^{+}$has similar interaction with the three phenyl rings, so bond distances written just for one phenyl ring. Bold text indicates the presence of coordination based on the sum of van der Waal radii that is Tl $\left(2.08^{216}\right)+\mathrm{C}\left(1.70^{215}\right)=3.78 \AA$.

3.17 Metal-carbon distances in $p \mathrm{Cp}$-tin complexes. In $p \mathrm{Cp} 1_{\mathrm{Ge}}$, the $\mathrm{M}-\mathrm{C}$ bond distances are shown for two top of the cavity carbon atoms of each of the three arene rings. The data for the experimentally known $\mathrm{GeCl}^{+}$ (Figure $1.8^{117}$ ) is also given for comparison. The Ge-C distances in this experimentally known complex vary over the top (T), bottom (B) and bridgehead $(\mathrm{BH})$ phenyl carbon atoms of the cavity in a relatively broader range compared to the identical Ge-C distances in $\mathrm{D}_{3}$ symmetric optimized $p \mathrm{Cp}-\mathrm{Ge}^{2+}$ geometry. In the calculated $p \mathrm{Cp}-\mathrm{Ge}^{2+}$, the metal ion has similar interaction with the three phenyl rings, so bond distances are not repeated. Bold text indicates the presence of coordination. Based on the sum of van der Waal radii that is $\mathrm{Ge}\left(2.11^{213}\right)$ $+\mathrm{C}\left(1.70^{215}\right)=3.81 \AA$ shows that all the M-C distances are classified as coordination but the visual representation of the Group $14 p \mathrm{Cp} 1_{\mathrm{M}}$ complexes shows otherwise. Hence, the classification of coordination in $p \mathrm{Cp} 1_{\mathrm{Ge}}$ based on three-dimensional view. . . . . . . . . .

3.18 Selected experimental bond lengths and dihedral angles in $p \mathrm{Cp}-\mathrm{GeCl}^{+}$.$\mathrm{Al}_{4} \mathrm{Cl}_{10} \mathrm{O}_{2}{ }^{2}-\mathrm{vs}$ their corresponding calculated values in $p \mathrm{Cp}-\mathrm{Ge}^{2+}$. ' $\mathrm{r}$ ' is for bond length $(\AA)$ and $\phi$ reflects a dihedral angle (degrees). . . . . .

3.19 Metal-carbon distances in $p$ Cp-tin complexes. In $p C p 1_{\mathrm{Sn}}$, the M-C bond distances are shown for two top of the cavity carbon atoms of each of the three arene rings. The data for the experimentally known $\mathrm{GeCl}^{+}$(Figure 1.8 ${ }^{117}$ ) is also given for comparison. The Ge-C distances in this experimentally known complex vary over the top (T), bottom (B) and bridgehead (BH) phenyl carbon atoms of the cavity in a relatively broader range compared to the identical Ge-C distances in $\mathrm{D}_{3}$ symmetric optimized $p \mathrm{Cp}-\mathrm{Sn}^{2+}$ geometry. In the calculated $\mathrm{Sn}^{2+}$ has similar interaction with the three phenyl rings, so bond distances have been noted down once. Bold text indicates the presence of coordination. Based on the sum of van der Waal radii that is Sn $\left(2.17^{213}\right)+\mathrm{C}$ $\left(1.70^{215}\right)=3.87 \AA$ shows that all the M-C distances are classified as coordination but the visual representation of $p C p 1_{\mathrm{Sn}}$ shows otherwise. Hence, the classification of coordination is based on three-dimensional view. . . . . . . . . . . . . . . . . . .

3.20 Selected experimental bond lengths and dihedral angles in $p \mathrm{Cp}-\mathrm{Sn}^{2+}$.$2 \mathrm{AlCl}_{4}{ }^{-} .2 \mathrm{C}_{6} \mathrm{H}_{6}$ vs their corresponding calculated values in $p \mathrm{Cp}-\mathrm{Sn}^{2+}$. ' $\mathrm{r}$ ' is for bond length $(\AA)$ and $\phi$ reflects a dihedral angle (degrees). . . . . 
3.21 Metal-carbon distances in $p \mathrm{Cp}$-lead inclusion complexes. In $p \mathrm{Cp} 2 \mathrm{~Pb}$, the M-C bond distances are shown for two top of the cavity carbon atoms of each of the three arene rings. $\mathrm{Pb}^{2+}$ has similar interaction with the three phenyl rings, so bond distances written just for one phenyl ring. Bold text indicates the presence of coordination. Based on the sum of van der Waal radii that is $\mathrm{Pb}\left(2.02^{213}\right)+\mathrm{C}\left(1.70^{215}\right)=3.72 \AA$, shows that all the M-C distances are classified as coordination but the visual representation of the complexes shows otherwise. Hence, the classification of coordination is based on three-dimensional view. . . . .

3.22 Metal-carbon distances in $p \mathrm{Cp}$-arsenic inclusion complexes. In $p \mathrm{Cp} 3_{\mathrm{As}}$, the M-C bond distances are shown for two top of the cavity carbon atoms of each of the three arene rings. $\mathrm{As}^{3+}$ has similar interaction with the three phenyl rings, so bond distances written just for one phenyl ring. Bold text indicates the presence of coordination based on the sum of van der Waal radii that is As $\left(1.85^{213}\right)+\mathrm{C}\left(1.70^{215}\right)=3.55 \AA . p \mathrm{Cp} 1_{\mathrm{As}}$ is known experimentally, ${ }^{110}$ so its values are also given in parentheses. .

3.23 Selected experimental bond lengths and dihedral angles vs their corresponding calculated $\left(\mathrm{C}_{3}\right)$ values in $p \mathrm{Cp} 1_{\mathrm{As}}$. ' $\mathrm{r}$ ' is for bond length $(\AA)$ and $\phi$ reflects a dihedral angle (degrees) . . . . . . . . . . . .

3.24 Metal-carbon distances in $p \mathrm{Cp}$-antimony complexes. In $p \mathrm{Cp} 3_{\mathrm{Sb}}$, the $\mathrm{M}$ $\mathrm{C}$ bond distances are shown for two top of the cavity carbon atoms of each of the three arene rings. $\mathrm{Sb}^{3+}$ has similar interaction with the three phenyl rings, so bond distances written just for one phenyl ring. Bold text indicates the presence of coordination based on the sum of van der Waal radii that is $\mathrm{Sb}\left(2.06^{213}\right)+\mathrm{C}\left(1.70^{215}\right)=3.76 \AA . p \mathrm{Cp} 2_{\mathrm{Sb}}$ is known experimentally ${ }^{110}$ and its values given in parentheses for comparison which shows that the difference in $\mathrm{M}-\mathrm{C}$ distances in both experimental and computational structures is within the range of van der Waal coordination threshold and both exhibit similar coordination. . . . . . .

3.25 Metal-carbon distances in $p \mathrm{Cp}$-bismuth complexes. In $p \mathrm{Cp} 1_{\mathrm{Bi}}$, the $\mathrm{M}$ $\mathrm{C}$ bond distances are shown for two top of the cavity carbon atoms of each of the three arene rings. $\mathrm{Bi}^{3+}$ has similar interaction with the three phenyl rings, so bond distances written just for one phenyl ring. Bold text indicates the presence of coordination based on the sum of van der Waal radii that is $\mathrm{Bi}\left(2.07^{213}\right)+\mathrm{C}\left(1.70^{215}\right)=3.77 \AA . p \mathrm{Cp} 3_{\mathrm{Bi}}$ is known experimentally, therefore its structural data has also been incorporated in parentheses. All the experimental versus computational $\mathrm{M}-\mathrm{C}$ distances are within the range of sum of van der Waal radii, so both have similar coordination mode. . . . . . . . . . . . . . . . 
3.26 Enthalpies $(\mathrm{kcal} / \mathrm{mol})$ of formation of metal complexes with [2.2.2]paracyclophane $(p C p)$ in the reaction $p \mathrm{Cp}+\mathrm{NMCl}_{\mathrm{n}} \rightarrow p \mathrm{Cp}\left(\mathrm{MCl}_{\mathrm{n}}\right)_{\mathrm{N}}$. Each exclusion complex is represented by $p \mathrm{CpN}_{M}$ where $\mathrm{N}=1,2$ or 3 for $p \mathrm{Cp}\left(\mathrm{MCl}_{\mathrm{n}}\right), p \mathrm{Cp}\left(\mathrm{MCl}_{\mathrm{n}}\right)_{2}, p \mathrm{Cp}\left(\mathrm{MCl}_{\mathrm{n}}\right)_{3}$ respectively and $\mathrm{M}=\mathrm{Ga}$, In, $\mathrm{Tl}, \mathrm{Ge}, \mathrm{Sn}, \mathrm{Pb}, \mathrm{As}, \mathrm{Sb}$ and $\mathrm{Bi}$. 'n' represents the number of chlorides attached to the metal in each case and its value is 1, 2 and 3 for the metals of Group 13, 14 and 15 respectively. The inclusion complexes $p \mathrm{Cp}-\mathrm{M}^{\mathrm{n}+}$ contain $\mathrm{M}^{\mathrm{n}+}$ inside the cavity of $p \mathrm{Cp}$. They are formed as $p \mathrm{Cp}+\mathrm{MCl}_{\mathrm{n}} \rightarrow p \mathrm{Cp}-\mathrm{M}^{\mathrm{n}+}+\mathrm{nCl}^{-}$where ' $\mathrm{n}$ ' denotes 1,2 , and 3 for the metals of Group 13,14 and 15 respectively. . . . . . . . . . . . . 72

3.27 $\Delta \mathrm{H}_{\text {dissoc. }}$ of metal chlorides to yield the metal cation $\left(\mathrm{M}^{\mathrm{n}+}\right)$. It decreases with the increase in covalent radii down the group. All values in $\mathrm{kcal} / \mathrm{mol}$.

3.28 Results of the MZEDA analysis for M-Cyclophane $\left(\mathrm{M}=\mathrm{Ga}^{+}, \mathrm{In}^{+}, \mathrm{Tl}^{+}\right)$ complexes in $\mathrm{C}_{3}$ symmetry (results for the $\mathrm{D}_{3}$ transition structures in parentheses) at the PBE0/TZ2P level. The percentage shows the contribution of an energy term in the total attraction energy which is the sum of $\Delta E^{\text {ele }}$ and $\Delta E^{\text {orb }} . \Delta E^{\text {hybrid }}$ is the component of $\Delta E^{\text {orb }}$ expressed separately in the MZEDA results. All values in $\mathrm{kcal} \mathrm{mol}^{-1} \ldots \ldots$. . . . 76

3.29 Results of the MZEDA analysis for M-Cyclophane $\left(\mathrm{M}=\mathrm{Ge}^{2+}, \mathrm{Sn}^{2+}\right.$, $\mathrm{Pb}^{2+}$ ) complexes in $\mathrm{D}_{3}$ symmetry at the PBE0/TZ2P level. The percentage in parentheses shows the contribution of an energy term in the total attraction energy which is the sum of $\Delta E^{\text {ele }}$ and $\Delta E^{\text {orb }} . \Delta E^{\text {hybrid }}$ is the component of $\Delta E^{\text {orb }}$ expressed separately in the MZEDA results. $A_{1}$, $A_{2}$ and $E_{1}$ are the non-reducible terms in which $\Delta E^{\text {orb }}$ of a $\mathrm{D}_{3}$ structure can be decomposed. All values in $\mathrm{kcal} \mathrm{mol}^{-1}$. . . . . . . . . . . 76

3.30 Results of the MZEDA analysis for M-Cyclophane $\left(\mathrm{M}=\mathrm{As}^{3+}, \mathrm{Sb}^{3+}\right.$, $\mathrm{Bi}^{3+}$ ) complexes in $\mathrm{D}_{3}$ symmetry (Note that the $\mathrm{D}_{3}$ symmetric As complex is a transition structure. The results for the $\mathrm{C}_{3}$ symmetric minimum are given in parentheses) at the PBE0/TZ2P level. The percentage shows the contribution of an energy term in the total attraction energy which is the sum of $\Delta E^{\text {ele }}$ and $\Delta E^{\text {orb }} . \Delta E^{\text {hybrid }}$ is the component of $\Delta E^{\text {orb }}$ expressed separately in the MZEDA results. $A_{1}, A_{2}$ and $E_{1}$ are the non-reducible terms in which $\Delta E^{\text {orb }}$ of a $\mathrm{D}_{3}$ structure can be decomposed. All values in $\mathrm{kcal} \mathrm{mol}^{-1}$. . . . . . . . . . . . . . .

3.31 Key structural parameters of deltaphane. Data from Dp1 Ag is given in square brackets. ' $r$ ' is for bond-length $(\AA)$ and $\phi$ shows the dihedral

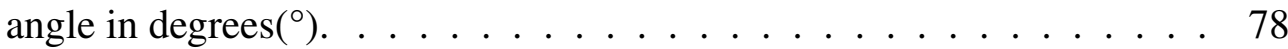


3.32 Metal-carbon distances in Dp-gallium exclusion complexes. In Dp1 $1_{\mathrm{Ga}}$, the M-C bond distances are shown for one top of the cavity carbon atom of each of the three arene rings. $\mathrm{Ga}^{+}$in $\mathrm{Dp}-\mathrm{Ga}^{+}$has similar interaction with the three phenyl rings, so bond distances written just for one phenyl ring. Bold text indicates the presence of coordination based on the sum of van der Waal radii ${ }^{213}$ that is $\mathrm{Ga}(1.87)+\mathrm{C}(1.70)=3.57 \AA .80$

3.33 Metal-carbon distances in Dp-indium exclusion complexes. In $\mathrm{Dp} 1_{\mathrm{In}}$, the M-C bond distances are shown for one top of the cavity carbon atom of each of the three arene rings. $\mathrm{In}^{+}$in $\mathrm{Dp}-\mathrm{In}^{+}$has similar interaction with the three phenyl rings, so bond distances written just for one phenyl ring. Bold text indicates the presence of coordination based on the sum of van der Waal radii that is In $\left(1.98^{214}\right)+\mathrm{C}\left(1.70^{215}\right)=3.68 \AA . \quad 83$

3.34 Metal-carbon distances in Dp-thalium exclusion complexes. In $\mathrm{Dp} 1_{\mathrm{Tl}}$, the $\mathrm{M}-\mathrm{C}$ bond distances are shown for one top of the cavity carbon atom of each of the three arene rings. $\mathrm{Tl}^{+}$in $\mathrm{Dp}-\mathrm{Tl}^{+}$has similar interaction with the three phenyl rings, so bond distances written just for one phenyl ring. Bold text indicates the presence of coordination based on the sum of van der Waal radii that is $\mathrm{Tl}\left(2.08^{216}\right)+\mathrm{C}\left(1.70^{215}\right)=3.78 \AA$.

3.35 Metal-carbon distances in Dp-germanium exclusion complexes. In Dp $1_{\mathrm{Ge}}$, the $\mathrm{M}-\mathrm{C}$ bond distances are shown for one top of the cavity carbon atom of each of the three arene rings. $\mathrm{Ge}^{2+}$ in $\mathrm{Dp}-\mathrm{Ge}^{2+}$ has similar interaction with the three phenyl rings, so bond distances written just for one phenyl ring. Bold text indicates the presence of coordination. The sum of van der Waal radii that is $\mathrm{Ge}\left(2.11^{216}\right)+\mathrm{C}\left(1.70^{215}\right)=3.81 \AA$ shows that all the M-C distances are classified as coordination but the visual representation of the complexes shows otherwise. Hence, the classification of coordination is based on three-dimensional view. . . . . . . . 84

3.36 Metal-carbon distances in Dp-tin exclusion complexes. In $\mathrm{Dp} 1_{\mathrm{Sb}}$, the $\mathrm{M}-\mathrm{C}$ bond distances are shown for one top of the cavity carbon atom of each of the three arene rings. $\mathrm{Sn}^{2+}$ in $\mathrm{Dp}-\mathrm{Sn}^{2+}$ has similar interaction with the three phenyl rings, so bond distances written just for one phenyl ring. Based on the sum of van der Waal radii that is $\mathrm{Sn}$ $\left(2.17^{213}\right)+\mathrm{C}\left(1.70^{215}\right)=3.87 \AA$ shows that all the M-C distances are classified as coordination but the visual representation of the complexes shows otherwise. Hence, the classification of coordination is based on three-dimensional view. . . . . . . . . . . . . . . . . . 84 
3.37 Metal-carbon distances in Dp-lead exclusion complexes. In $\mathrm{Dp} 1_{\mathrm{Pb}}$, the $\mathrm{M}-\mathrm{C}$ bond distances are shown for one top of the cavity carbon atom of each of the three arene rings. $\mathrm{Pb}^{2+}$ in $\mathrm{Dp}-\mathrm{Pb}^{2+}$ has similar interaction with the three phenyl rings, so bond distances written just for one phenyl ring. Based on the sum of van der Waal radii that is $\mathrm{Pb}$ $\left(2.02^{213}\right)+\mathrm{C}\left(1.70^{215}\right)=3.72 \AA$, shows that all the M-C distances are classified as coordination but the visual representation of the complexes shows otherwise. Hence, the classification of coordination is based on three-dimensional view. . . . . . . . . . . . . . .

3.38 Metal-carbon distances in Dp-arsenic exclusion complexes. In Dp $1_{\mathrm{As}}$, the M-C bond distances are shown for one top of the cavity carbon atom of each of the three arene rings. $\mathrm{As}^{3+}$ in $\mathrm{Dp}-\mathrm{As}^{3+}$ has similar interaction with the three phenyl rings, so bond distances written just for one phenyl ring. Bold text indicates the presence of coordination based on the sum of van der Waal radii that is As $\left(1.85^{213}\right)+C\left(1.70^{215}\right)$ $=3.55 \AA$.

3.39 Metal-carbon distances in Dp-antimony exclusion complexes. In Dp $1_{\mathrm{Sb}}$, the $\mathrm{M}-\mathrm{C}$ bond distances are shown for one top of the cavity carbon atom of each of the three arene rings. $\mathrm{Sb}^{3+}$ in $\mathrm{Dp}-\mathrm{Sb}^{3+}$ has similar interaction with the three phenyl rings, so bond distances written just for one phenyl ring. Bold text indicates the presence of coordination based on the sum of van der Waal radii that is $\mathrm{Sb}\left(2.06^{213}\right)+\mathrm{C}\left(1.70^{215}\right)=3.76 \AA .86$

3.40 Metal-carbon distances in Dp-bismuth complexes. In Dp1 $1_{\mathrm{Bi}}$, the M-C bond distances are shown for one top of the cavity carbon atom of each of the three arene rings. $\mathrm{Bi}^{3+}$ in $\mathrm{Dp}-\mathrm{Bi}^{3+}$ has similar interaction with the three phenyl rings, so bond distances written just for one phenyl ring. Bold text indicates the presence of coordination based on the sum of van der Waal radii that is $\operatorname{Bi}\left(2.07^{213}\right)+\mathrm{C}\left(1.70^{215}\right)=3.77 \AA$. . . . 86

3.41 Enthalpies $(\mathrm{kcal} / \mathrm{mol})$ of formation of metal complexes with deltaphane $(\mathrm{Dp})$ in the reaction $\mathrm{Dp}+\mathrm{NMCl}_{n} \rightarrow \mathrm{Dp}\left(\mathrm{MCl}_{\mathrm{n}}\right) \mathrm{N}$. Each exclusion complex is represented by $\mathrm{DpN}_{\mathrm{M}}$ where $\mathrm{N}=1,2$ or 3 for $\mathrm{Dp}\left(\mathrm{MCl}_{\mathrm{n}}\right), \mathrm{Dp}\left(\mathrm{MCl}_{\mathrm{n}}\right)_{2}$, $\mathrm{Dp}\left(\mathrm{MCl}_{\mathrm{n}}\right)_{3}$ respectively and $\mathrm{M}=\mathrm{Ga}, \mathrm{In}, \mathrm{Tl}, \mathrm{Ge}, \mathrm{Sn}, \mathrm{Pb}, \mathrm{As}, \mathrm{Sb}$ and $\mathrm{Bi}$. $n$ represents the number of chlorides attached to the metal in each case and its value is 1, 2 and 3 for the metals of Group 13, 14 and 15 respectively. The inclusion complexes Dp- $\mathrm{M}_{\mathrm{n}}{ }^{+}$contain $\mathrm{M}_{\mathrm{n}}{ }^{+}$in the centre of the cavity of Dp. They are formed as $\mathrm{Dp}+\mathrm{MCl}_{\mathrm{n}} \rightarrow \mathrm{Dp}-\mathrm{M}_{\mathrm{n}}^{+}+\mathrm{nCl}^{-}$ where $n$ denotes 1,2 , and 3 for the metals of Group 13, 14 and 15 respectively. . . . . . . . . . . . . . . 
3.42 Results of the MZEDA analysis for M-Deltaphane $\left(\mathrm{M}=\mathrm{Ga}^{+}, \mathrm{In}^{+}, \mathrm{Tl}^{+}\right)$ complexes in $\mathrm{D}_{3}$ symmetry (results for the $\mathrm{C}_{3}$ minimum structures in parentheses) at the PBE0/TZ2P level. The percentage shows the contribution of an energy term in the total attraction energy which is the sum of $\Delta E^{\text {ele }}$ and $\Delta E^{\text {orb }} . \Delta E^{\text {hybrid }}$ is the component of $\Delta E^{\text {orb }}$ expressed separately in the MZEDA results. $A_{1}, A_{2}$ and $E_{1}$ are the non-reducible terms in which $\Delta E^{\text {orb }}$ of a $\mathrm{D}_{3}$ structure can be decomposed. All values in $\mathrm{kcal} \mathrm{mol}^{-1}$.

3.43 Results of the MZ-EDA analysis for M-Deltaphane $\left(\mathrm{M}=\mathrm{Ge}^{2+}, \mathrm{Sn}^{2+}\right.$, $\mathrm{Pb}^{2+}$ ) complexes in $\mathrm{D}_{3}$ symmetry (results for the $\mathrm{C}_{3}$ minimum structures in parentheses) at the PBE0/TZ2P level. The percentage shows the contribution of an energy term in the total attraction energy which is the sum of $\Delta E^{\text {ele }}$ and $\Delta E^{\text {orb }} . \Delta E^{\text {hybrid }}$ is the component of $\Delta E^{\text {orb }}$ expressed separately in the MZEDA results. $A_{1}, A_{2}$ and $E_{1}$ are the nonreducible terms in which $\Delta E^{\text {orb }}$ of a $\mathrm{D}_{3}$ structure can be decomposed.

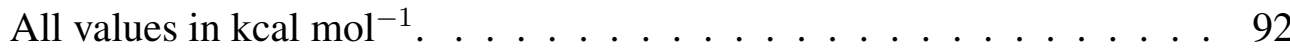

3.44 Results of the MZEDA analysis for M-Deltaphane $\left(\mathrm{M}=\mathrm{As}^{3+}, \mathrm{Sb}^{3+}\right.$, $\mathrm{Bi}^{3+}$ ) complexes in $\mathrm{D}_{3}$ symmetry (The results for the $\mathrm{C}_{3}$ symmetric minimum are given in parentheses) at the PBE0/TZ2P level. The percentage shows the contribution of an energy term in the total attraction energy which is the sum of $\Delta E^{\text {ele }}$ and $\Delta E^{\text {orb }} . \Delta E^{\text {hybrid }}$ is the component of $\Delta E^{\text {orb }}$ expressed separately in the MZEDA results. $A_{1}, A_{2}$ and $E_{1}$ are the non-reducible terms in which $\Delta E^{\text {orb }}$ of a $\mathrm{D}_{3}$ structure can be decomposed. All values in $\mathrm{kcal} \mathrm{mol}^{-1} \ldots \ldots \ldots \ldots$. . . . . . .

4.1 QTAIM analysis of some important bonds in InZn-1 and InZn-2. $\rho\left(\mathrm{r}_{\mathrm{BCP}}\right)$ denotes electron density along a bond critical path (BCP), $\nabla^{2} \rho\left(\mathrm{r}_{\mathrm{BCP}}\right)$ represents Laplacian of the electron density, $\mathrm{G}\left(\mathrm{r}_{\mathrm{BCP}}\right) / \rho\left(\mathrm{r}_{\mathrm{BCP}}\right)$ shows the ratio of kinetic energy to the electron density and $\mathrm{H}\left(\mathrm{r}_{\mathrm{BCP}}\right)$ shows electronic local energy density. Reprinted from reference ${ }^{217}$ with permission (Licence ID: 1001280-1) from the publisher. Copyright (2019) Royal Society of Chemistry. . . . . . . . . . . . . . . . . . 100

5.1 Key parameters from the ground state structures both in gas phase and in solvent calculated at PBE0-D3BJ/def2TZVP level in the current study. $\mathrm{C} 7=\mathrm{O} 12$ is the carbonyl of benzanthrone, C3-N13 corresponds to the bond linking the amino substituents to the benzanthrone nucleus, $\phi$ reflects the dihedral angle (degrees) for the plane C2-C3-N13-C14. $\mu$ shows dipole moment (in debyes). The crystal structure data of Compound (38) ${ }^{166}$ is given for comparison as its geometry resembles that of $1_{\mathrm{AB}}$ with slight modifications on the substituent. . . . . . . . . . . 104

5.2 NBO charges of some significant atoms . . . . . . . . . . 108 
5.3 Band gap calculated as a change in SCF energy between the gas and ethanol phase optimized frontier molecular orbitals. All energies in eV. . 109

5.4 Results of Vertical excitations highlighting absorbed energy $\left(\Delta \mathrm{E}_{\text {abs }}\right)$ in $\mathrm{eV}$, oscillator strength $(f)$ and dipole moment $(\mu)$ in debye for the first excited state. . . . . . . . . . . . . . . . 111

5.5 Dipole moment of ethanol-solvated dyes in their ground state $\mu_{\mathrm{GS}}$ and in excited state $\mu_{\mathrm{ES}} \ldots \ldots \ldots \ldots \ldots \ldots$

5.6 Results of excited state optimization highlighting the energy emitted $\left(\Delta \mathrm{E}_{\text {ems }}\right)$ in $\mathrm{eV}$, oscillator strength $(f)$ and dipole moment $(\mu)$ in debye for the first excited state. $\phi$ reflects the dihedral angle (degrees) for the plane C2-C3-N13-C14 . . . . . . . . . . . . . . . . . 115

5.7 Dipole moment of ethanol-solvated dyes on their absorption maxima $\mu_{\text {abs }}$ and after relaxation of the excited states to their minima $\mu_{\mathrm{ems}}$. Stokes shift (S.S.) can be calculated as the difference of the absorbed energy as a result of vertical excitation and emission energy resulting from relaxation of the excited state minimum back to the ground state .117

5.8 Comparison of key parameters from the solvated phase ground state and excited state structures of the dyes under discussiom. $\mathrm{C} 7=\mathrm{O} 12$ is the carbonyl of benzanthrone, C3-N13 corresponds to the bond linking the amino substituents to the benzanthrone nucleus, $\phi$ reflects the dihedral angle (degrees) for the plane C2-C3-N13-C14. $\mu$ shows dipole moment (in debyes) . . . . . . . . . . . . . . . . . . 118

5.9 A comparison of the excitation energies $\left(\Delta \mathrm{E}_{\mathrm{abs}}\right)$ in $\mathrm{eV}$ for the first excited singlet and triplet states. . . . . . . . . . . . . . . . 119 


\section{Glossary}

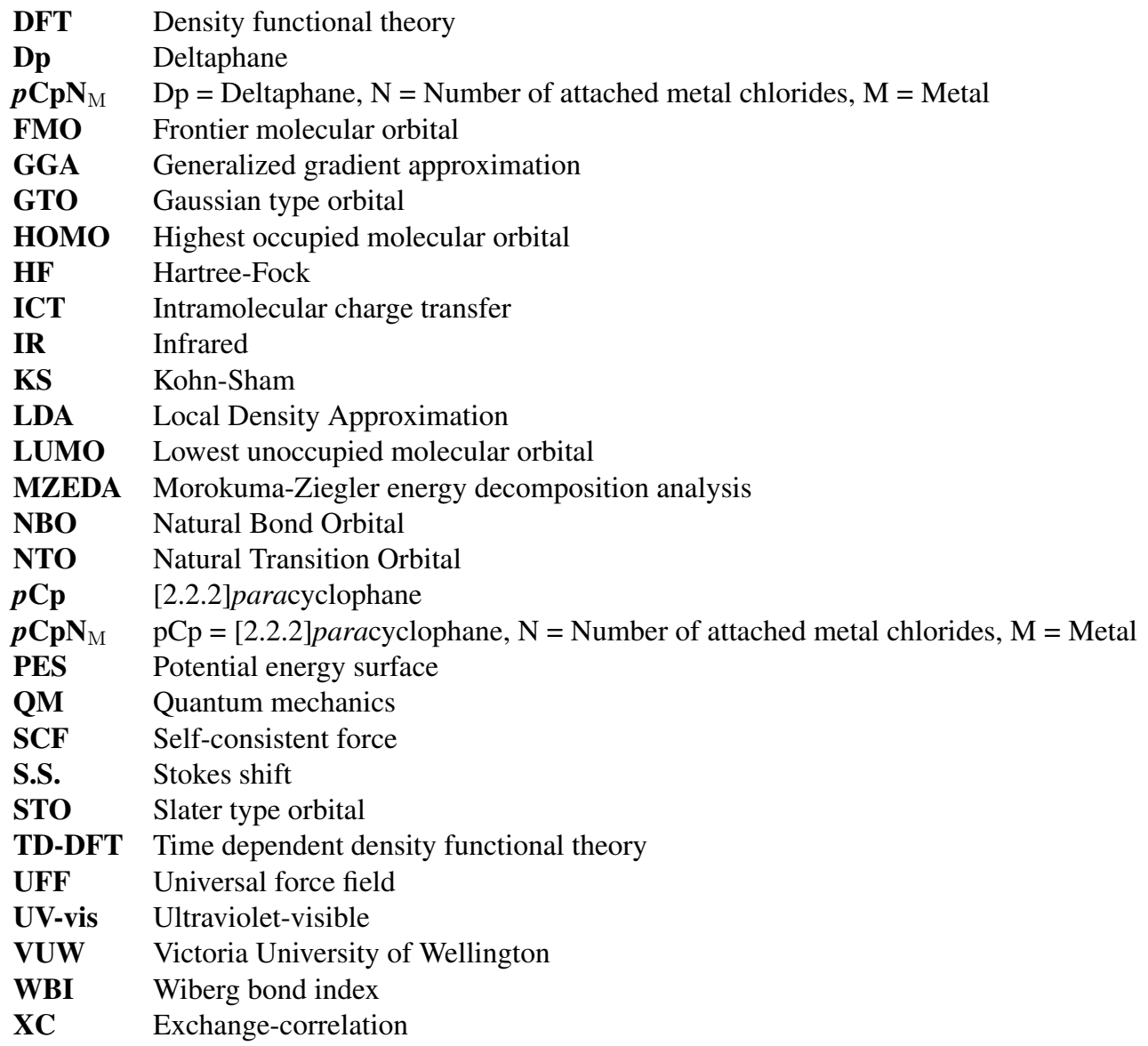




\section{Chapter 1}

\section{Introduction}

Donor-acceptor interactions are the attractive forces between two components of a chemical system that involve donation of charge or a lone pair of electrons from one component to the other. ${ }^{1,2}$ These attractive forces play an important role in metal-ligand coordination complexes ${ }^{3,4}$ and intermolecular ${ }^{5,6}$ as well as intramolecular systems. ${ }^{7-9}$ The mode of bonding in coordination complexes is a well-known phenomenon. As a general rule, one or more ligands donate their $\pi$-electrons to an electropositive center i.e. a metal atom or a metallic cation thus giving rise to donor-acceptor bonding. The transition metals in turn may release the excess negative charge by donating electrons from their $d$-orbitals to the ligand empty $\pi$ orbitals of the same symmetry. This latter phenomenon is called $\pi$-backbonding. However, this rule is not followed always; metallic complexes may exist where a metal-guest ( $\pi$-acceptor) is hosted by a $\pi$-rich system thus giving rise to host-guest coordination. Intermolecular interactions have been known and studied for a long time in different forms such as dipole-dipole forces, London dispersion forces ${ }^{10}$ and hydrogen bonding. ${ }^{11,12}$ In the so called intramolecular donor-acceptor systems, electronic charge flows from comparatively electropositive (donor) poles towards electronegative (acceptor) regions as a result of electronic excitation. ${ }^{9}$ Such molecules with intramolecular charge transfer bear excellent photophysical properties such as fluorescence. ${ }^{13}$ The current computational study deals with 
the donor-acceptor compounds of two types: (1) coordination complexes of heavier main-group metals and (2) benzanthrone dyes with intramolecular donor-acceptor interactions.

The advent of supercomputers and their involvement in the study of chemical processes gave rise to a new field of chemistry called computational chemistry. It is now possible to model structures and reactions to investigate their experimental feasibility rather than passing through unsuccessful exhaustive laboratory exercises. Computational chemistry also confirms already predicted structures and reaction mechanisms and in this way, it can help point out erroneous assumptions made in old works.

There has been a notable decline in computational financial cost with the passage of time. According to Moore's Law, the number of transistors on an integrated circuit gets doubled biennially ${ }^{14}$ which shows how fast computational power is increasing with the passing of time. However, it is more interesting to know that the financial value of a 1 gigaflops computation in 1960 was 7.9 trillion US dollars whereas in today's currency it is less than 20 cents. ${ }^{15}$ Depending on available computational facilities and resources, now-a-days, it is possible to obtain successful simulation results with any desired accuracy. However, it is a general observation that energies for smaller systems can be calculated more accurately compared to the larger ones (with higher number of electrons and in turn the atoms).

\subsection{Heavier Main-Group Metal Complexes}

The heavier main group metals of Groups 13 (Ga, In, Tl), 14 (Ge, Sn, Pb) and 15 (As, $\mathrm{Sb}, \mathrm{Bi}$ ) have gained considerable attention over the late twentieth century. ${ }^{16}$ Transition metal-like behaviour of these main-group elements provoked insights into the properties ${ }^{17-19}$ of their compounds which, in many cases, helped synthesize new main-groupmetal compounds that have interesting features. ${ }^{20}$ Since the 1970 s, there has been an 
increased interest in studying the synthesis, bonding and physical properties of such complexes. $^{21}$

Many of the complexes containing heavier main-group metals are catalytically active. ${ }^{22}$ Especially the compounds of bismuth have roles as promoters in selective oxidation, ${ }^{23,24}$ oxidation/ammoxidation, ${ }^{25}$ cyclization ${ }^{26}$ reactions, and many more. Small-molecule activation has been one of the key interests of chemists for the last few decades. Compounds containing heavier main-group metals provide an alternative to expensive catalysts for activation. ${ }^{27}$ Complexes of the lower main-group metals are also known for their applications in synthesis of polymers ${ }^{28}$ and materials. ${ }^{29}$ Group 14 lower metals are well-known to form analogues of alkenes ${ }^{30,31}$ and alkynes. ${ }^{32}$ Due to their relatively low toxicity compared to transition metals, compounds of bismuth are also used in biomedicine. ${ }^{33-35}$ There is a growing interest in the host-guest interactions of late maingroup elements ${ }^{36-38}$ due to the potential applications of such interactions in metal chelation $^{39}$ and ion-selective electrodes. ${ }^{40}$

Metals of Groups 1, 2, 13, 14 and 15 are called main-group metals. The last half century has witnessed lot of development in the chemistry of these metals and currently a large number of main-group metal complexes have been shown to exist. ${ }^{41-49}$ These main-group elements have also been found to be useful for synthetic work; for example, they can be an alternative to transition metals in catalysis. ${ }^{50,51}$ There is a search for aromaticity in main-group metal clusters with interesting properties and applications. ${ }^{52,53}$ Scientists are always interested to know about binding modes ${ }^{54,55}$ of metals and the factors that affect the bonding. ${ }^{16,56-58}$

\subsubsection{Transition Metal-like Behaviour}

It is undoubtedly the fact that transition metals have a richer chemistry in terms of their complex formation and reactivity when compared to main-group metals. However, the last 50 years have witnessed significant developments featuring the formation of heav- 
ier main-group-metal compounds that resemble transition metal compounds in their behavior. Some important classes of such compounds are ethene analogues, ${ }^{59} \mathrm{com}-$ plexes with pincer ligands, ${ }^{60}$ compounds having both main-group metals and transition metals ${ }^{61}$ and very interestingly, the ones with donor-acceptor (including host-guest) interactions. ${ }^{4}$ Host-guest complexes include the examples where a $\pi$-efficient component of a system donates $\pi$-electrons to the acceptor component. ${ }^{62-65}$. In the next few subsections, we shall briefly focus on the complexes of Groups 13, 14 and 15 late metals particularly keeping in view their donor-acceptor perspective.

\subsubsection{Group 13 Compounds}

Sandwich complexes containing $\mathrm{Ga}^{+}, \mathrm{In}^{+}$and $\mathrm{Tl}^{+}$in coordination with $\mathrm{P}_{\mathrm{n}}$ and $\mathrm{As}_{\mathrm{n}}$ ligands are also known to undergo coordination polymerization. It is already established that the pattern of reactivity of $\mathrm{P}_{\mathrm{n}}$ ligand complexes is different to their $\mathrm{As}_{\mathrm{n}}$ analogues. ${ }^{66}$ However, in one of their studies, Fleischmann and co-workers reported similar behaviour of cyclo- $\mathrm{P}_{5}$ and cyclo- $\mathrm{As}_{5}$ ligands by synthesizing their complexes with group 13 metal cations. They explained the coordination behaviour in these complexes through various means including DFT. ${ }^{67}$ The overall reaction is a simple one, resulting in the coordination polymerization product as shown in Scheme 1.1.

The product from the reaction in Scheme 1.1 shows that there are three $\mathrm{P}_{5}$ or $\mathrm{As}_{5} \pi$ bonded rings around a metal cation, each with $\eta^{5}$ coordination to the corresponding cation. All these compounds were found to be fairly soluble in methylene chloride having a dynamic coordination in the solution while they decomposed in donor solvents such as MeCN. DFT calculations were employed to further investigate energy changes during the reaction and the coordination behaviour in these complexes. Unlike other $\eta^{5}$ complexes successfully isolated in the solid state, solid coordination polymers containing $\mathrm{Ga}^{+}$ions with cyclo- $\mathrm{As}_{5}$ ligands could not be obtained and this may be attributed to the fact that the ionic radius of $\mathrm{Ga}^{+}$is smaller compared to its heavier group members. ${ }^{68}$ Computationally optimized geometries in this study suggest that the smaller ionic ra- 

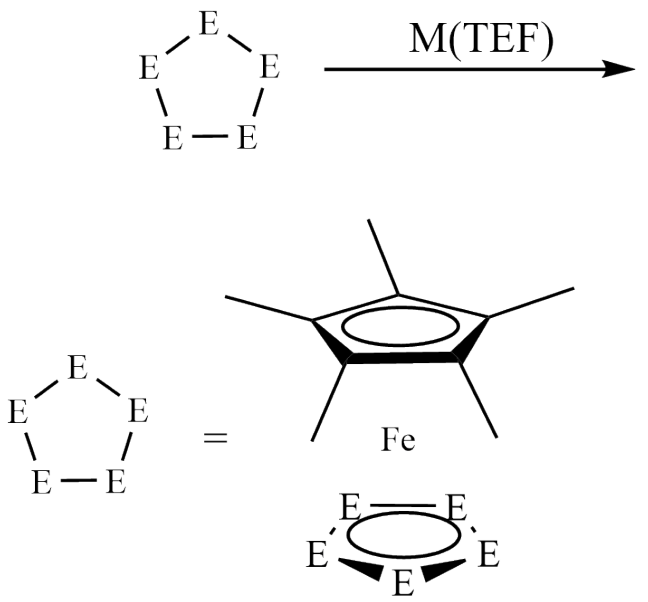

$\left[\mathrm{Cp}^{*} \mathrm{FeE}_{5}\right]$ where $\mathrm{Cp}^{*}=\mathrm{C}_{5} \mathrm{Me}_{5}$

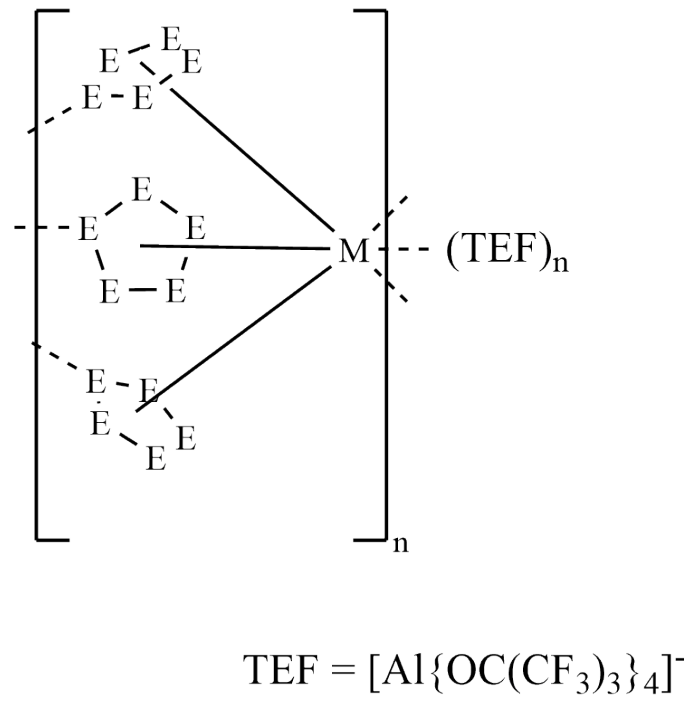

1 (a): $\mathrm{E}=\mathrm{P}$

1 (b): $\mathrm{E}=\mathrm{As}$
i) $\mathrm{M}=\mathrm{Ga}$
i) $\mathrm{M}=\mathrm{In}$
ii) $\mathrm{M}=\mathrm{In}$
ii) $\mathrm{M}=\mathrm{Tl}$
iii) $\mathrm{M}=\mathrm{Tl}$

Scheme 1.1: Sandwich complexes of Group 13 lower $\mathrm{M}^{+}$cations with TEF. Reprinted with permission from reference ${ }^{66}$. Copyright (1999) American Chemical Society.

dius renders $\pi$-interactions shorter in length which in turn cause steric crowding and it is not possible for three cyclic ligands to form $\eta^{5}$ coordination to $\mathrm{Ga}^{+}$.

Formazanido ligands have recently been found capable of interacting with some of the Group 13 metals. ${ }^{69}$ Derivatives of formazans are known for their role in synthesizing dyes and as indicators for detection of metals ions. ${ }^{70,71}$ Most of these complexes, however, were known with transition metals and, to some extent, with alkali metals until recently when their first synthesis involving aluminium, gallium and indium was reported. ${ }^{69}$ The formation of $\mathrm{M}(1,3,5$-triphenylformazan $) \mathrm{Me}_{2}$ resulted when the trimethyl metal derivatives $\left(\mathrm{MMe}_{3}\right)$ were allowed to interact with 1,3,5-triphenylformazan (Scheme 1.2). The reactions for all the three metals were reported to be carried out separately in toluene at room temperature. These complexes show excellent photophysical properties. Their UV-visible spectra show a bathochromic shift compared to the 1,3,5triphenylformazan starting ligand. This might be due to the negative charge on the ligand in complexes as opposed to its starting neutral form. The red shift is increas- 
ingly pronounced in this case compared to the boron analogue. ${ }^{72}$ Moreover, a significant HOMO-LUMO gap was found in these complexes. This makes them interesting candidates to be studied further for their photophysical characteristics.

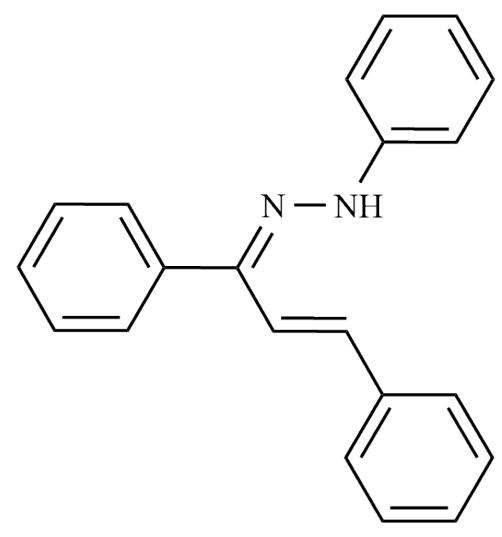

1,3,5-triphenylformazan

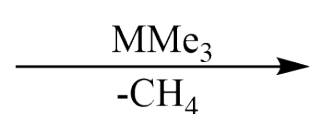

(3) $\mathrm{M}=\mathrm{Ga}$

(4) $\mathrm{M}=$ In

Scheme 1.2: Synthesis of Group 13 formazanido complexes. Reprinted with permission from reference ${ }^{69}$ - Published by The Royal Society of Chemistry under Creative Commons Attribution-NonCommercial 3.0 Unported Licence.

\subsubsection{Group 14 Complexes}

These main-group metals complexes have been being studied for a long time due to their potential to form heterobimetallic complexes with transition metals. ${ }^{73}$ They also have interesting photo-catalytic ${ }^{74}$ and physical properties. ${ }^{75}$ Due to the ability to change their oxidation state in a variety of complexes, ${ }^{76,77}$ people are also interested in studying the binding modes of Group 14 metals. ${ }^{78-80}$ These metals are especially known for their unsaturated hydrocarbon analogues. ${ }^{81,82}$ A stable digermavinylidene (Figure 1.1 has been reported recently. ${ }^{83}$ This development rejected the general concept about methylidene carbine $\left(\mathrm{H}_{2} \mathrm{C}=\mathrm{C}\right.$ : $)$ and its analogues that were once known to be just short-lived intermediates at high temperature and pressure. ${ }^{84,85}$ Crystallographic data reported for this new compound (5) shows 1,1-disubstituted $\mathrm{Ge}_{2}$ in which two germanium atoms are covalently bonded (as the bond length between these two is short enough to indicate a double bond). Both Ge atoms and the B atoms are coplanar. The B-Ge (1) bond lengths 
were reported to be consistent with a tri-coordinate geometry. DFT investigations were also reported. It was found that digermavinylidene, that is cis in terms of orientation of the substituents around $\mathrm{Ge}=\mathrm{Ge}$, is more stable compared to its trans isomer. ${ }^{83}$ However, the reverse is true for bulky aromatic substituents and the trans isomer in that case is much more stable. ${ }^{86}$

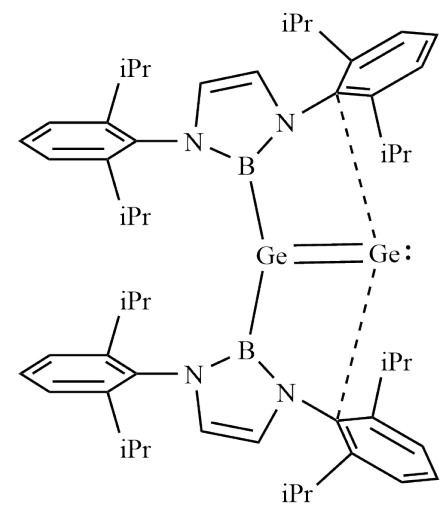

(5)

Figure 1.1: A stable digermavinylidene

The stabilization of Group 14 metals, in various oxidation states, has also gained attraction due to their potential applications such as the synthesis of semiconductors. ${ }^{87,88}$ The use of transition metal ligands as acceptors and NHC ligands as donors for this purpose has been an interesting choice. ${ }^{89}$ Rivard's group extended the idea from their previous studies $^{90}$ and synthesized methylene analogues of the hydrides of Ge and Sn (Scheme 1.3). ${ }^{89}$ These complexes were further characterized with different spectroscopic techniques. The Group 14 metal-tungsten $\left(\mathrm{M}_{14}-\mathrm{W}\right)$ bond bond was considerably longer which shows the absence of $\pi$-bonding. It can be inferred that this interaction is best described as a single bond. Both complexes were found to be thermally stable. DFT studies of model complexes assisted in analyzing the nature of different interactions within (6) and (7). The $\mathrm{M}_{14}-\mathrm{W}$ bond was reported to be due to lone pair donation from $\mathrm{M}$ to the empty $\sigma^{*}$ orbitals of $\mathrm{W}-\mathrm{C}$ in both the cases. Accordingly, the $\mathrm{M}-\mathrm{W}-\mathrm{C}_{\text {trans }}$ is shown linear in their structures. 


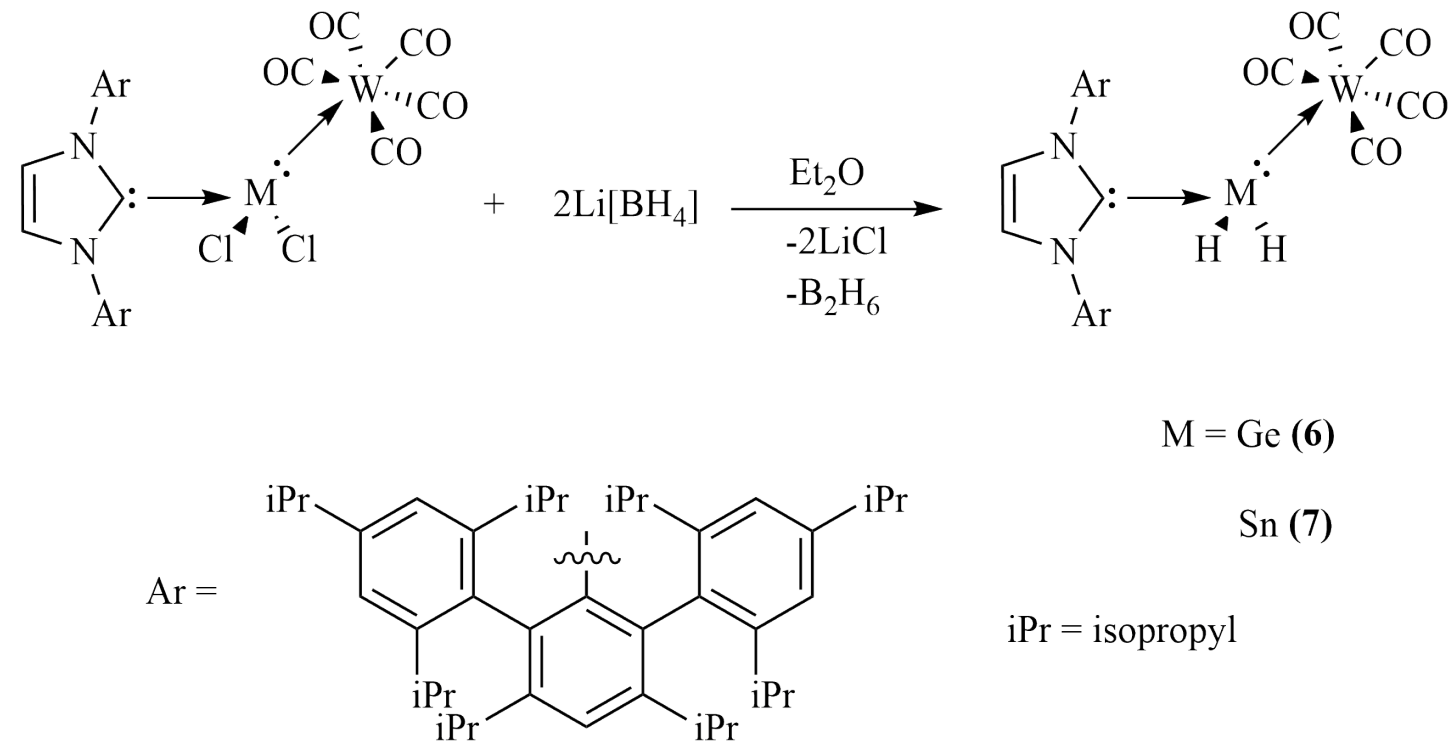

Scheme 1.3: Synthesis of $\mathrm{GeH}_{2}$ and $\mathrm{SnH}_{2}$ analogues. NHC moiety acts as a donor liginad while pentacarbonyl tungsten $(0)$ is acceptor ligand. Reprinted with permission from reference ${ }^{89}$. Copyright (2010) American Chemical Society.

\subsubsection{Group 15 Complexes}

Arsenic, antimony and bismuth have a rich coordination chemistry. Their complexes find applications in ionic liquids, ${ }^{91}$ catalysis, ${ }^{25}$ material science, ${ }^{92} \mathrm{P}_{4}$ activation, ${ }^{27,93}$ organic synthesis ${ }^{94}$ and biomedicine. ${ }^{95,96}$ The donor-acceptor complexes of these Group 15 heavy metals have been known for a long time. ${ }^{97}$ This is also evident from the synthesis of some trialkyl aluminium adducts and study of their structure and stability. ${ }^{98}$ These were $\left(\mathrm{C}_{2} \mathrm{H}_{5}\right)_{3} \mathrm{AlAs}\left(\mathrm{SiMe}_{3}\right)_{3} \mathbf{( 8 )}$ and $\left(\left(\mathrm{CH}_{3}\right)_{3} \mathrm{C}\right)_{3} \mathrm{AlAs}\left(\mathrm{C}\left(\mathrm{CH}_{3}\right)_{2}\right)_{3}$ (9) complexes (Figure 1.2) that were prepared and their structures were further analyzed by X-ray studies. It was reported that the As-Al bond was longer than expected. This indicates dative bond character in the first case while in the latter, an even longer bond distance was observed. This was reported as the first ever distant interaction between As and Al. (8) and (9) are Lewis acid-base adduct type complexes where $\mathrm{AsR}_{3}$ exhibits basicity and the alane part is acidic in nature. The coordination properties of these complexes and the model analogues of $\mathrm{Sb}$ and $\mathrm{Bi}$ were further studied through computational means.

The thermal stability values calculated computationally were reported to follow the 


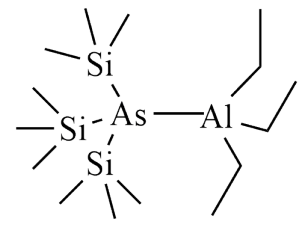

(8)

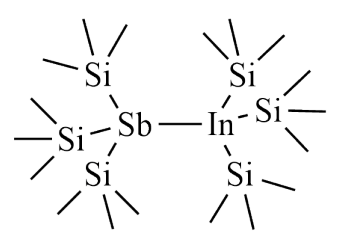

(11)

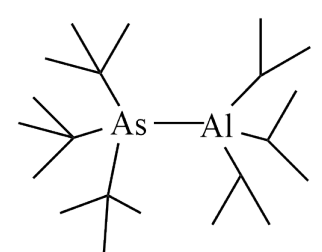

(9)

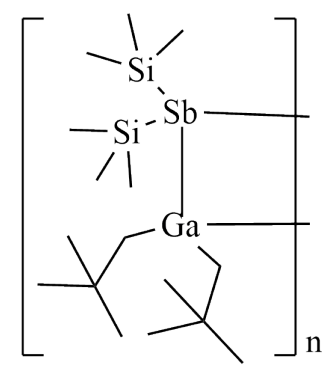

(12)

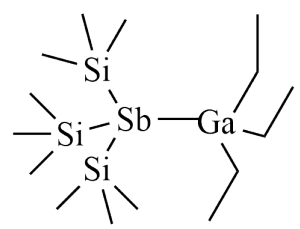

(10)

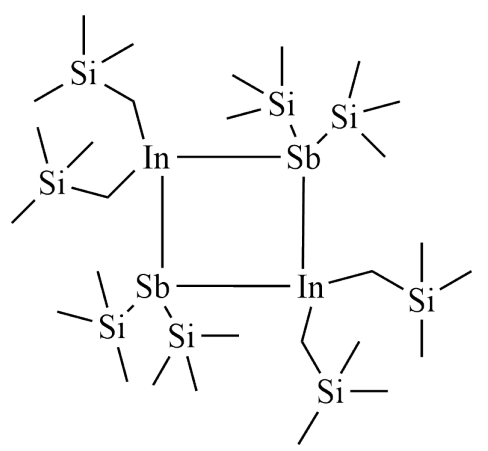

(13)

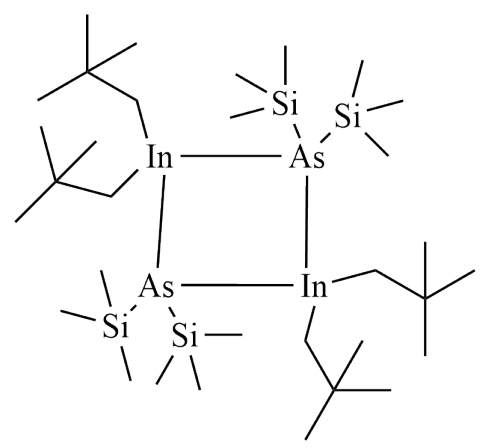

(14)

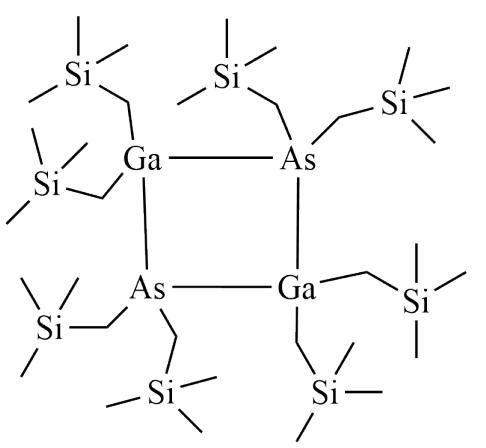

(15)

Figure 1.2: Lewis acid-base type complexes of Group 15 metals with that of Group 13 in which M15 acts as donor and M13 behaves as acceptor

Haaland model of thermodynamic stability, which states that the greater the $\mathrm{C}-\mathrm{Al}$ bond length in an alane, the more stable it will be. ${ }^{99}$ Dissociation energies were underestimated while overestimations in the bond lengths and bond angles were encountered. Although it was a notable achievement in connection to one of their previous works, ${ }^{100}$ the authors themselves admit the limitations of their computational methodology. Hence despite a good reflection of the trends of thermal stability found in these complexes, there is room for improved study of the computational part in this work.

Kuckzowski et al. ${ }^{98}$ took their inspiration from elsewhere ${ }^{101}$ to go through a range of experiments including those mentioned above. Although the idea to investigate the 
possibility of M13-M15 interactions was published previously, ${ }^{102}$ it was Wells' group who pioneered isolation and characterization of such complexes in search of the potential applications of these compounds in semiconductor materials. ${ }^{103-105}$ Antimonides of Group 13 are an extension to this series ${ }^{101}$ of the type $\mathrm{R}_{n} \mathrm{M} 13-\mathrm{SbR}_{n}$ with different $\mathrm{R}$ and R' substituents as shown in Figure 1.2. All four complexes, (10) to (13) were reported to be prepared at room temperature and were successfully crystallized at $-30^{\circ} \mathrm{C}$. However, the crystals of (12) were not able to be analyzed through X-ray crystallography due to their thermo-photosensitivity. So, its structure was determined with the help of mass spectrometry and NMR. Compounds (10) and (11) were reported as the first ever examples of a dative bond of antimony with gallium or indium. The crystallographic data for dimeric (35) confirmed a planar four membered ring of alternating In and $\mathrm{Sb}$ atoms. The $\mathrm{Sb}-\mathrm{Ga}$ bond length was found to be less than that in Sb-In which can be attributed to the smaller atomic size of Ga. Sb-In in (13) is $3.0 \AA$ compared to 2.8 ̊ bond length of Sb-Ga bond.

Other complexes containing M15-E13 interactions include, for example, compounds (14) ${ }^{104}$ and (15). ${ }^{106}$ Both were reported in dimeric forms in which (14) has a planar, four-membered ring of alternating As and In atoms. The AsInAs and InAsIn bond angles were reported to be $83.46^{\circ}$ and $96.54^{\circ}$ respectively. This is in accordance with the general assumption that the endocyclic bond angle on a Group 13 metal is smaller than that on a metal of Group 15. ${ }^{107}$ Complex (15) has also been reported in dimeric form. However, it is different from (14) in the sense that the four-membered ring of alternating As and Ga atoms is clearly non-planar as evident from its X-ray structure. The very small dihedral angles in the ring and small As-Ga bond distances show that (15) is a strained molecule. The existence of the dimer in solution was confirmed through cryoscopic molecular mass determination. The dimerization was, however, reported to be fluxional on increase of the temperature. This was evident from the broadening and again sharpening of the ${ }^{13} \mathrm{C}$ NMR peaks. Compound (15) was thermally unstable at ambient temperature and started decomposing to $\left(\mathrm{Me}_{3} \mathrm{Si}\right)_{2} \mathrm{CH}_{2} \mathrm{As}$ and some other unknown products ${ }^{106}$. 


\subsubsection{Metal-Cyclophane Host-Guest Complexes}

Cyclophanes are molecules comprising two or more aromatic rings bridged by aliphatic chains. ${ }^{108}$ The aliphatic components cause a strain in the geometry which renders cyclophanes non-planar. ${ }^{109}$ In smaller cyclophanes the strain is even more pronounced, which may cause electronic cloud repulsion toward the outer surfaces of aromatic rings Therefore, metal cations can coordinate with the lighter cyclophanes to form exclusion complexes, ${ }^{64}$ but comparatively larger cyclophanes with bigger cavities form both exclu$\operatorname{sion}^{110}$ and inclusion ${ }^{111}$ complexes. Such donor-acceptor complexes of cyclophanes are receiving attention due to various applications including metal-ion chelation, ${ }^{39}$ catalysis ${ }^{40}$ and the development of ion-selective electrodes. ${ }^{112}$ Among these cyclophanes are the $\pi$-prismands [2.2.2]paracyclophane $(p \mathrm{Cp})^{113}$ and deltaphane systematically known as [2.2.2.2.2.2] $(1,2,4,5)$ cyclophane $(D p)^{63}$ both shown in Figure 1.3 whose first ever syntheses were reported in two separate studies. Later on, a high resolution crystal structure of $p \mathrm{Cp}$ was reported as $p \mathrm{Cp} . \mathrm{C}_{6} \mathrm{~N}_{4}$ (Figure 1.4). ${ }^{114}$ Both $p \mathrm{Cp}$ and Dp consists of three aromatic rings but differ in the number of ethyl bridges connecting the rings. In $p \mathrm{Cp}$, each aromatic ring is connected to two ethyl chains from its para positions whereas Dp contains four ethyl chains connected to each phenyl ring.
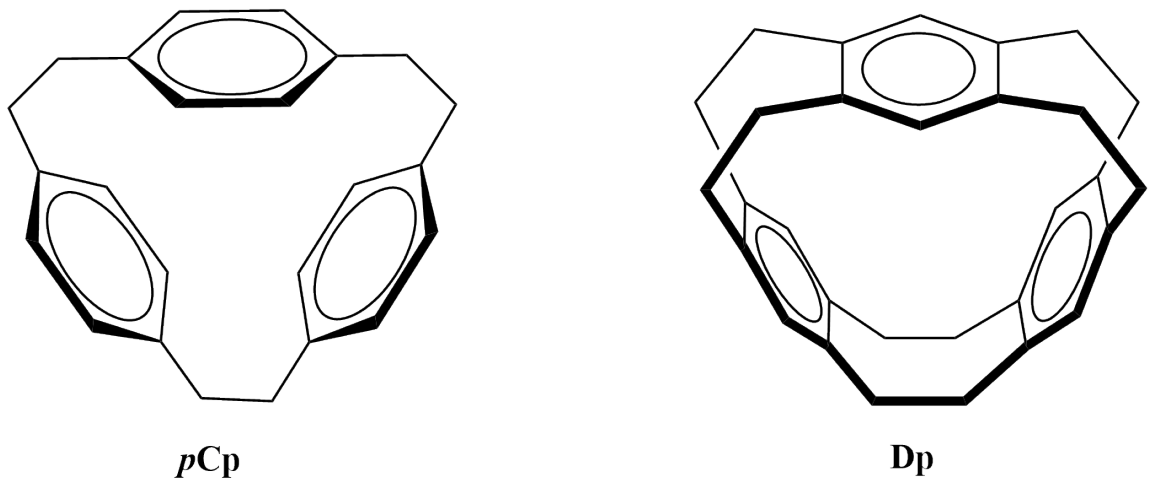

Figure 1.3: Cyclophane ligands of prism-shaped cavity capable of hosting electrondeficient metals. These are also the ligands of our interest in the study of coordination of main-group cyclophane complexes. $p \mathrm{Cp}$ represents [2.2.2]paracyclophane and Dp denotes deltaphane.

The complexes of cyclophanes with transition metals have been known for a long time. ${ }^{62,63,115}$ Kang et al. reported a coordination complex of silver triflate with deltaphane. ${ }^{63}$ 

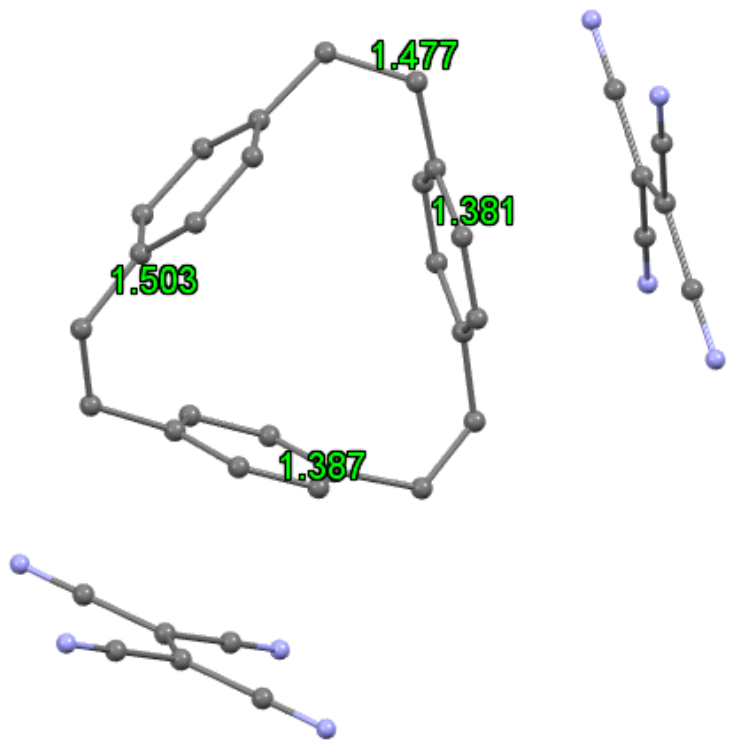

Figure 1.4: Crystal structure of $p$ Cp. $\mathrm{C}_{6} \mathrm{~N}_{4}\left(\mathrm{CSD} 1129300^{114}\right)$

Here, the silver atom is located over the top of the deltaphane cavity as shown in its crystal structure in Figure 1.5.

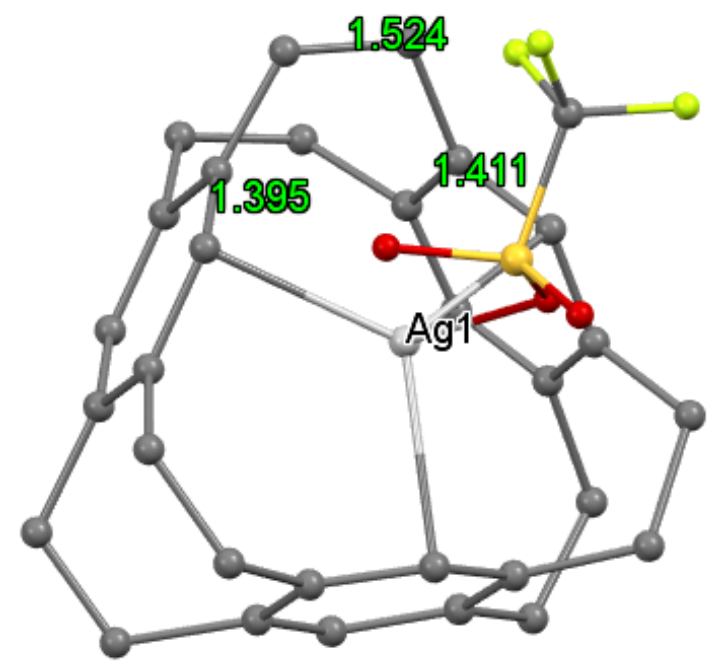

Figure 1.5: Crystal structure of Dp-Ag ${ }^{+}\left(\mathrm{CSD} 1134190^{63}\right)$

Schmidbaur et al. first started exploring heavier main group metal-complexes of cyclophanes. ${ }^{65}$ They allowed [2.2]paracyclophane to react with $\mathrm{Ga}\left[\mathrm{GaBr}_{4}\right]$ and obtained (16) which is an exclusion complex i.e. $\mathrm{Ga}^{+}$makes $\eta^{6} \cdot \eta^{6}$ coordination with the external surface of the rings (Figure 1.6). Complex (16) was reported as dimeric in solution. 
However, $\mathrm{In}^{+}$and $\mathrm{Tl}^{+}$were reluctant to form such a complex. Very little precipitation was reported as a result of the attempt to synthesize the $\mathrm{In}^{+}$-complex while $\mathrm{Tl}^{+}$ remained completely unaffected in the corresponding reaction.

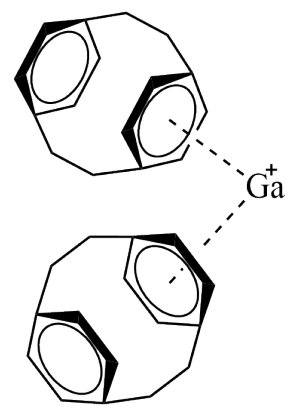

(16)

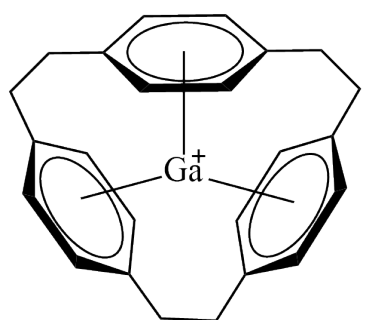

(17) $=p \mathrm{Cp}-\mathrm{Ga}^{+}$

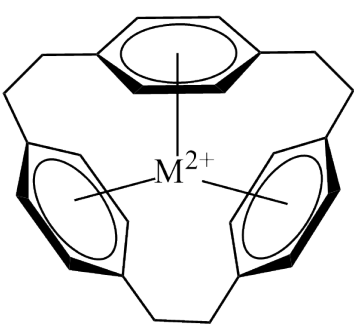

(18) $=p \mathrm{Cp}-\mathrm{Ge}^{2+}(\mathrm{M}=\mathrm{Ge})$

(19) $=p \mathrm{Cp}-\mathrm{Sn}^{2+}(\mathrm{M}=\mathrm{Sn})$

Figure 1.6: Results of some pioneering studies regarding cyclophane complexes with main-group metals

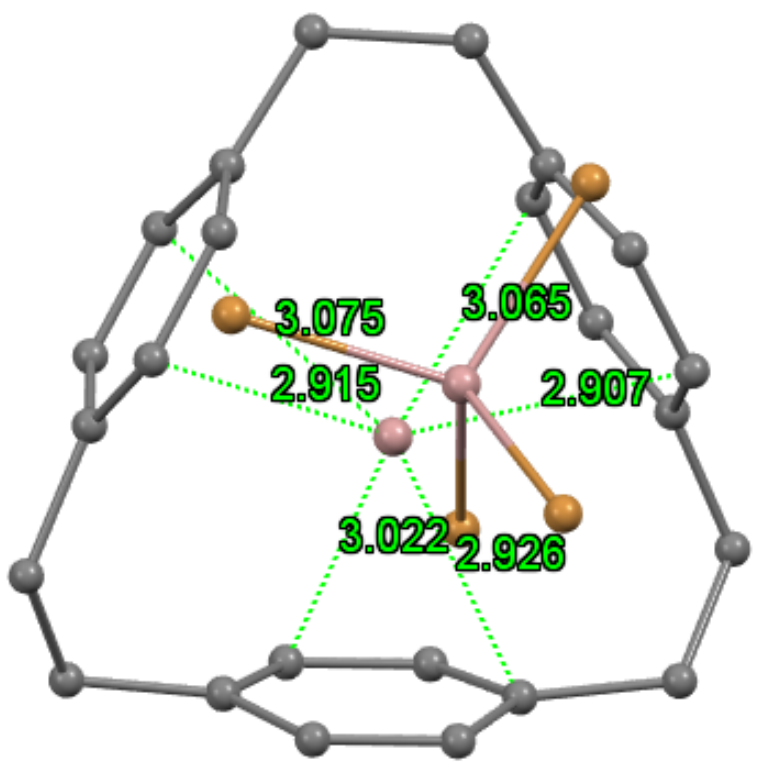

Figure 1.7: Crystal structure of $p \mathrm{Cp}-\mathrm{Ga}_{\mathrm{GaBr}}\left(\mathrm{CSD} 1156091^{116}\right)$

Later on, in a similar reaction they used $p \mathrm{Cp}$ as a ligand instead of [2.2]paracyclophane and obtained an inclusion complex (17) denoted as $p \mathrm{Cp}-\mathrm{Ga}^{+}$where $\mathrm{Ga}$ was $\eta^{6} \cdot \eta^{6} \cdot \eta^{6}$ coordinated with the three benzene rings. ${ }^{116}$ The crystal structure of (17) given in Figure 1.7 shows that $\left[\mathrm{GaBr}_{4}\right]^{-}$is a counter-anion that is in coordination with Ga present inside 
the $p \mathrm{Cp}$ cavity. The range of metal-carbon (M-C) distances given in Figure 1.7 shows that the gallium atom is equidistant from all the 18 carbon atoms of the $p \mathrm{Cp}$ cavity. Complex (17) was reported as the first in which a metal cation is located right in the center of the cyclophane cavity.

Complexes (18) denoted as $p \mathrm{Cp}-\mathrm{Ge}^{2+}$ and (19) represented as $p \mathrm{Cp}-\mathrm{Sn}^{2+}$ were also reported ${ }^{117}$ by the same group. The crystal structure of (18) in Figure 1.8 is in the form of $p \mathrm{Cp}-\mathrm{GeCl}^{+} .\left(\mathrm{Al}_{4} \mathrm{Cl}_{10} \mathrm{O}_{2}\right)^{2-} .2 \mathrm{C}_{6} \mathrm{H}_{6}$ and shows that $\mathrm{Ge}$ is bound to one chloride that forces the germanium atom tending to come toward the top of the cavity as shown by the range of M-C distances while still in coordination with all the 18 carbon atoms of the $p \mathrm{Cp}$ cavity. However, (19) was reported to crystallize as $p \mathrm{Cp}-\mathrm{Sn} .2 \mathrm{AlCl}_{4} \cdot 2 \mathrm{C}_{6} \mathrm{H}_{6}$ (Figure 1.9 where the tin atom is more strongly coordinated with the $p \mathrm{Cp}$ cavity compared to its germanium analogue, that is suggested by the range of M-C distances too.
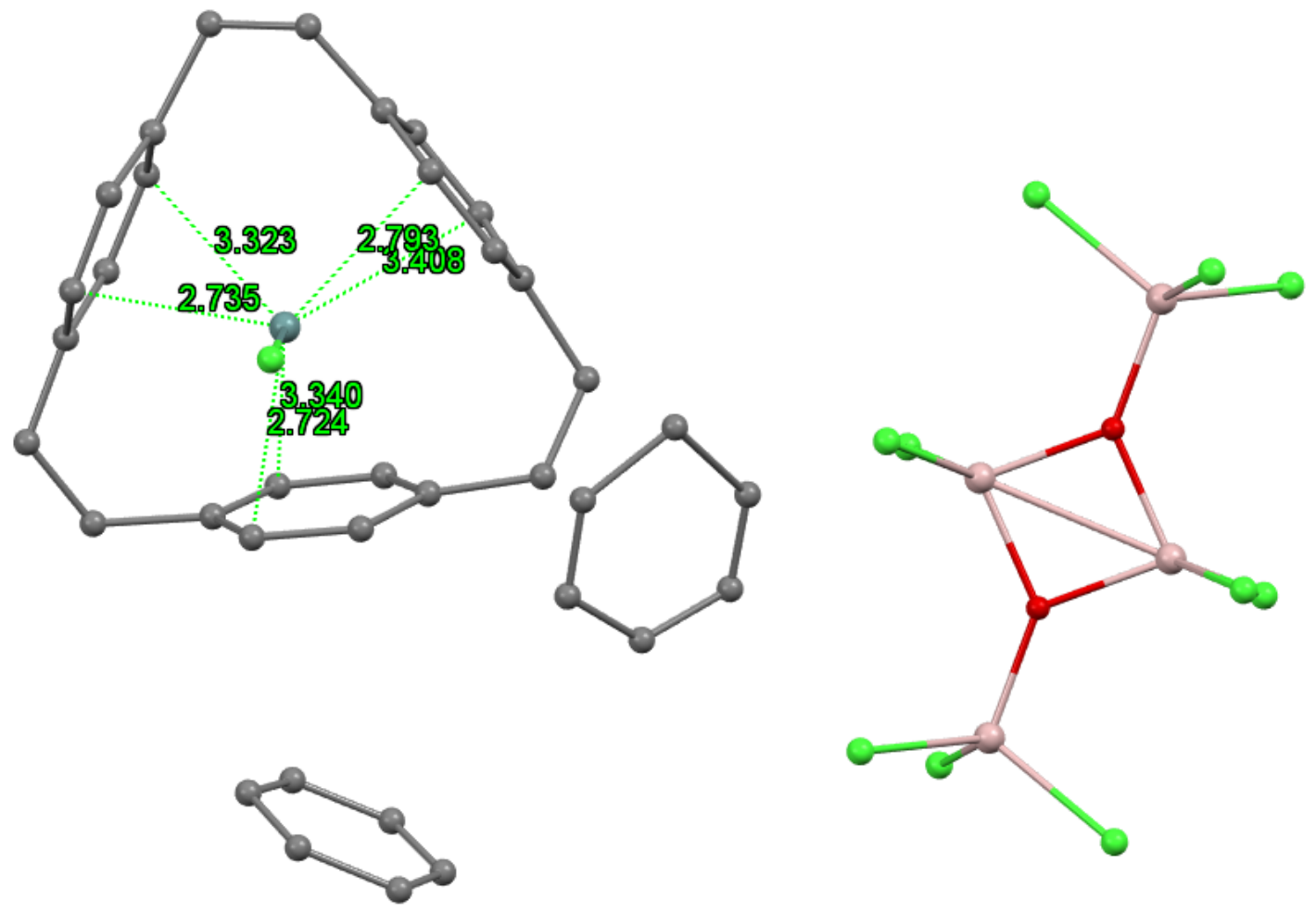

Figure 1.8: Crystal structure of $p \mathrm{Cp}-\mathrm{GeCl}^{+} .\left(\mathrm{Al}_{4} \mathrm{Cl}_{10} \mathrm{O}_{2}\right)^{2-} .2 \mathrm{C}_{6} \mathrm{H}_{6}\left(\mathrm{CSD} 1196799^{117}\right)$

Schmidbaur et al. kept searching for main-group metal complexes with $p \mathrm{Cp}$ and reported the synthesis of three Group 15 metal chloride complexes. ${ }^{110}$ One of them was 

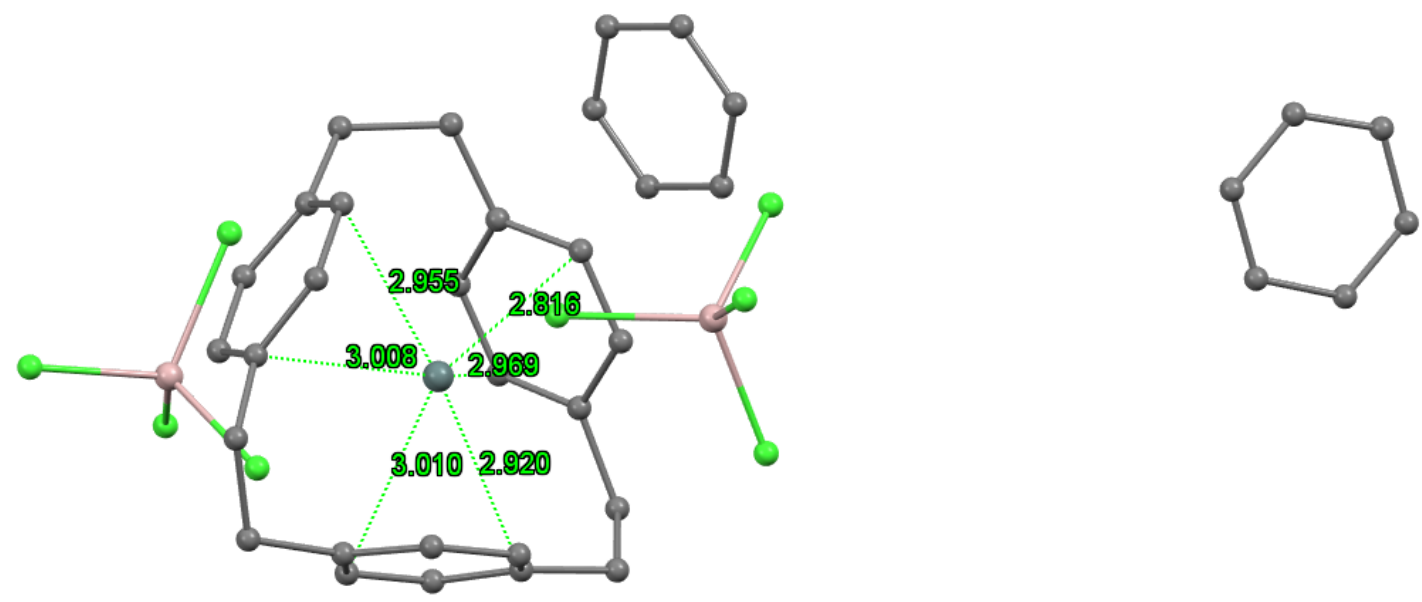

Figure 1.9: Crystal structure of $p \mathrm{Cp}-\mathrm{Sn} .2 \mathrm{AlCl}_{4} \cdot 2 \mathrm{C}_{6} \mathrm{H}_{6}\left(\mathrm{CSD} 1196798^{117}\right)$

the inclusion complex, $p \mathrm{Cp} 1_{\mathrm{As}}$ where $\mathrm{AsCl}_{3}$ approaches the $p \mathrm{Cp}$ from top of the cavity (Figure 1.10) interacting with two carbon atoms of each aromatic ring. The other two were the exclusion complexes of $\mathrm{SbCl}_{3}$ (Figure 1.11) and $\mathrm{BiCl}_{3}$ (Figure 1.12). For our convenience, we name them as $p \mathrm{Cp} 2_{\mathrm{Sb}}$ and $p \mathrm{Cp} 3_{\mathrm{Bi}}$ respectively because of the fact that two phenyl rings of $p \mathrm{Cp}$ are occupied by the two molecules of $\mathrm{SbCl}_{3}$ from outside in the former case while in the latter three $\mathrm{BiCl}_{3}$ molecules occupy all three phenyl rings. Although, the range of $\mathrm{M}-\mathrm{C}$ distances in all these complexes is quite large, they all are still within the sum of metal-carbon van der Waal radii to be considered as coordination.

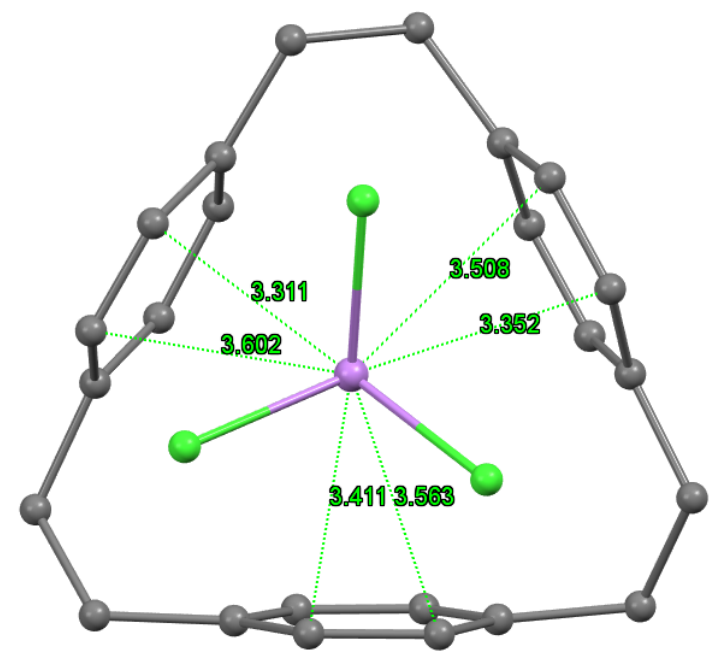

Figure 1.10: Crystal structure of (20) $p \mathrm{Cp}_{\mathrm{As}}\left(\mathrm{CSD} 1197586^{110}\right)$

Complexes of main-group metals are also known with modified cyclophanes. In a study 

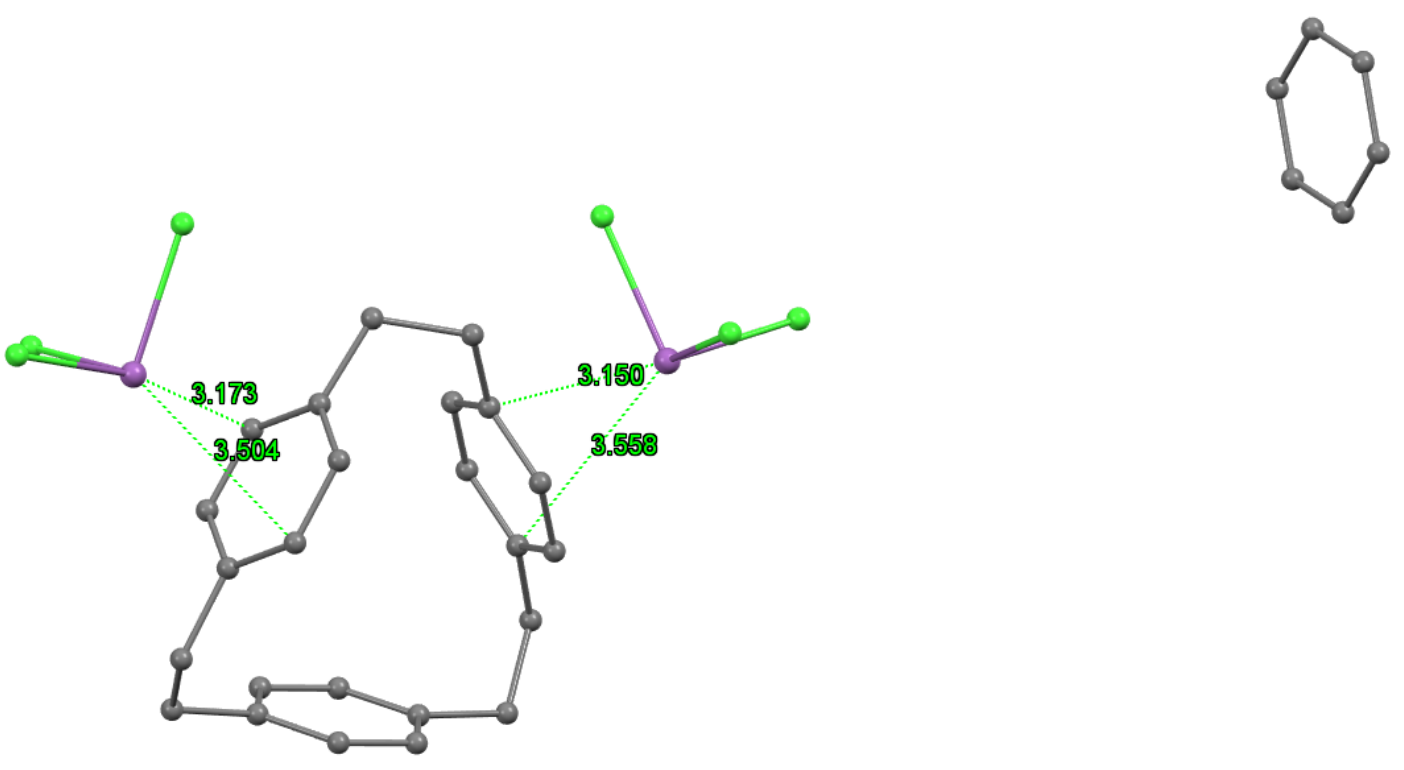

Figure 1.11: Crystal structure of (21) $p \mathrm{Cp} 2_{\mathrm{Sb}}\left(\mathrm{CSD} 1197587^{110}\right)$
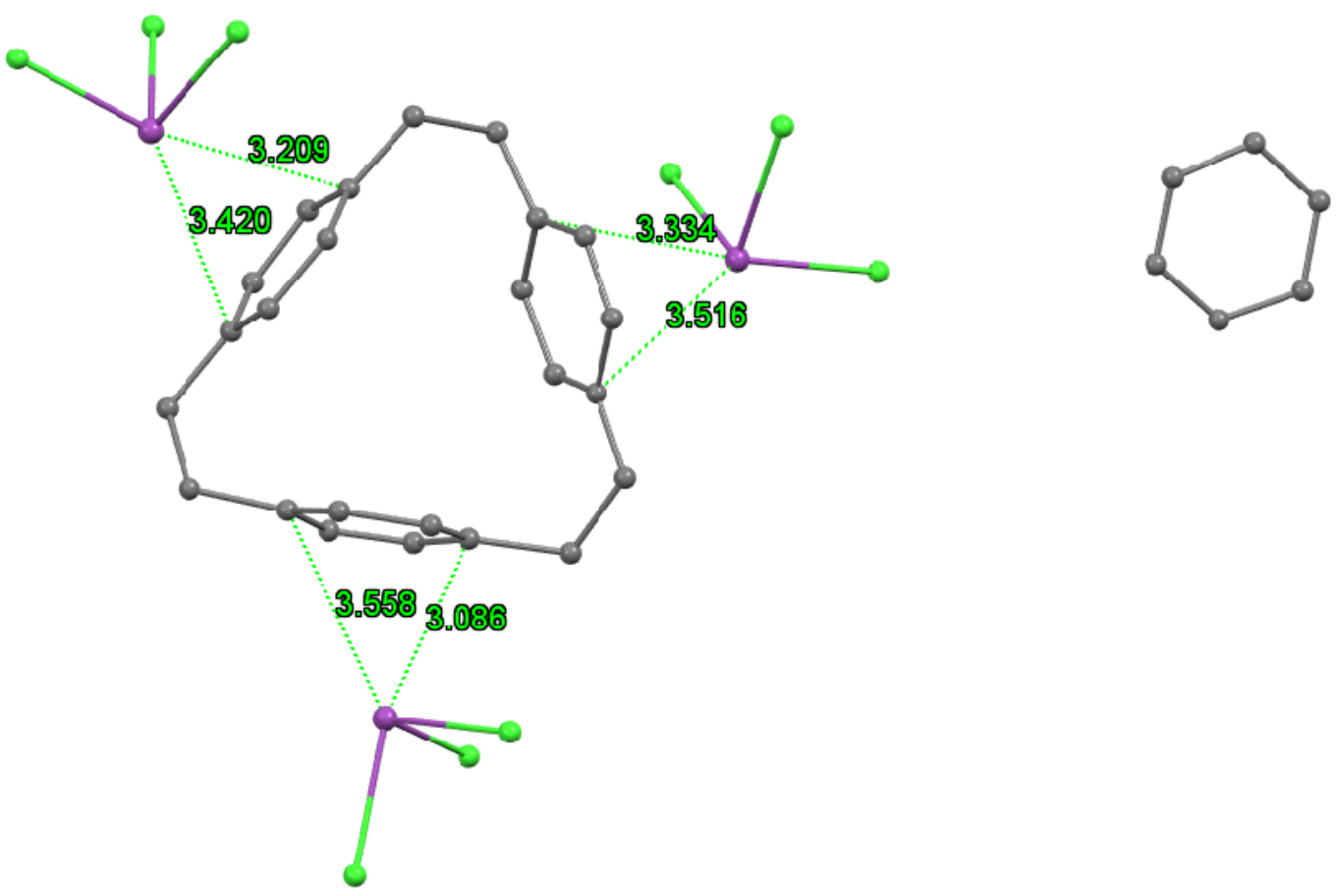

Figure 1.12: Crystal structure of (22) $p \mathrm{Cp} 3_{\mathrm{Bi}}\left(\mathrm{CSD} 1197588^{110}\right)$

conducted by Kunze et al., ${ }^{118} \mathrm{Ga}^{+}$and $\mathrm{Tl}^{+}$were found to form stable complexes with a nitrogen containing cyclophane (Figure 1.13). The strong coordination of $\mathrm{M}^{+}$with three arene rings and with two $\mathrm{N}$ atoms was reported to enhance the stability of (23). This five-way coordination in a trigonal bipyramid was thought responsible for the fact that the complex did not react with water, oxygen or polar solvents. DFT calculations 
helped understand the molecular orbital picture of (23). The optimized geometries were in agreement with the experimental data. NBO analysis further clarified the electronic structure. It was reported that the interaction between $\mathrm{M}^{+}$and the aromatic rings was high compared to that between $\mathrm{M}^{+}$and the $\mathrm{N}$ atoms. The HOMO was shown to be the result of a linear combination between the metal $s$ orbital and the two nitrogen lone pairs. The LUMO and the LUMO-1 were mainly $\pi$ in character with some contribution from the metal $p$ orbitals.

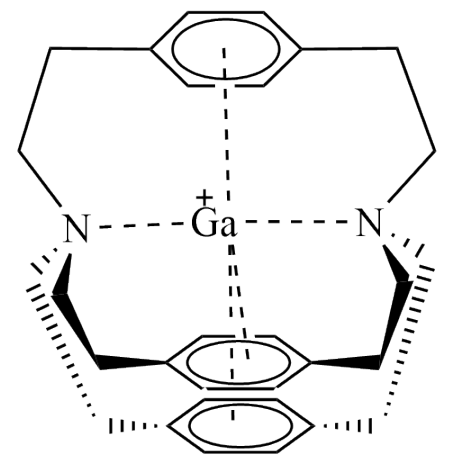

(23)

Figure 1.13: Trigonal bipyramidal coordination of $\mathrm{Ga}^{+}$

The above discussion shows that there are two major types of main-group metal cyclophane complexes. Those having the coordination of a metal chloride with the outer surfaces of the aromatic rings are called exclusion complexes as seen in the case of $p \mathrm{Cp} 2_{S b}$ and $p \mathrm{Cp} 3_{B i}$. The complexes where the core of the cyclophane cavity is involved may be referred to as inclusion complexes such as $p C p 1_{A s}$ and $p C p-M^{\mathrm{n}+}$. This means that inclusion complexes are of further two types. In one, the cation interacts with the three arene rings to occupy the center of the cavity and all the 18 carbon atoms of the 3 arene rings may interact equally with it. ${ }^{117}$ This is called $\eta^{6} \cdot \eta^{6} \cdot \eta^{6}$ or as a whole $\eta^{18}$ interaction. The second type has the metal chloride residing on top of the cavity and only two (in $p \mathrm{Cp}$ ) or one (in $\mathrm{Dp}$ ) carbon atoms from each ring contribute to the coordination with it forming an $\eta^{2} \cdot \eta^{2} \cdot \eta^{2}$ or $\eta^{1} \cdot \eta^{1} \cdot \eta^{1}$ binding mode in case of $p \mathrm{Cp}$ and Dp respectively. Recently, [2.2.2] paracyclophane ( $p \mathrm{Cp}$ ) host-guest complexes of $\mathrm{Ag}^{+}$ with $\eta^{2} \cdot \eta^{2} \cdot \eta^{2}$ coordination and $\mathrm{Sn}^{2+}$ with a $\eta^{6} \cdot \eta^{6} \cdot \eta^{6}$ interaction mode were compared 
computationally to explore the differences between the two modes of coordination. ${ }^{119}$ The reported computationally optimized geometries were in agreement with the experimental results. ${ }^{113,117}$ A smaller energy difference between $5 s$ orbital of $\mathrm{Ag}+$ and frontier orbitals of $p \mathrm{Cp}$ was shown to be the reason for exclusion complex formation between the two. In contrast, the $5 p$ orbital of $\mathrm{Sn}^{2+}$ was suggested to have major contribution to the interaction with the $\pi$ system of $p \mathrm{Cp}$ which facilitated the central mode of coordination in $p \mathrm{Cp}-\mathrm{Sn}^{2+}$ complex.

As evident from the above discussion, some $\eta^{18}$ coordinated heavier main group metals with $p \mathrm{Cp}$ are reported in the literature. However, to the best of our findings, $p \mathrm{Cp}$ complexes with cations of all the metals under current study have not been characterized yet. It would, therefore, be very useful to do a comparative study of $p \mathrm{Cp}-\mathrm{M}^{\mathrm{n}+}$ complexes where $\mathrm{M}^{\mathrm{n}+}$ means the cations of heavier metals of Group 13, 14 and 15. Moreover, the complexes of these metals with a cyclophane having more ethano bridges linking the benzene rings than $p \mathrm{Cp}$ such as deltaphane (Dp) are missing. Some Dp-transition metal interactions have already been established experimentally ${ }^{63}$ and computationally. ${ }^{111}$ Hence, it will be interesting to extend this idea to the main group metals. To the best of our knowledge, the current study is first ever computational insight into $p \mathrm{Cp}$ and Dp guest-host complexes with these nine metals viz; Ga, In, Tl, Ge, Sn, Pb, As, Sb and Bi. Different possibilities will be the subject of our study to explore the stability of hypothetical inclusion and exclusion complexes in this regard. Both the ligands of our interest are shown in Figure 1.3.

\subsubsection{Bonding Properties}

Due to their role in complex formation like transition metals, heavier main-group metals have been extensively studied for their binding patterns and modes of interaction. ${ }^{99,120,121}$ One basic reason for their transition-metal like peculiar nature are their large covalent radii in contrast to their lighter $(\mathrm{B}, \mathrm{C}, \mathrm{N})$ homologues. ${ }^{16,120}$ Takagi et al. contributed in 
the search for alkyne analogues of Group 14 metals by suggesting the stability of some hypothetical digermynes and distannynes based on computational studies. ${ }^{86}$ Later, it was argued that a $\mathrm{Sn}-\mathrm{Sn}$ triple bond is only possible in a trans bent geometry, ${ }^{122,123}$ hence, the complexes of this type are called distannynes. The same is the case with many of the alkene analogues of Group 14 heavier metals where the existence of a double bond was reported. Structural investigations for such compounds suggested the presence of a dimeric metal-metal interaction. ${ }^{20,124}$ However, some people are of the view that such compounds are misleadingly called alkene and alkyne analogues because they decompose to their respective carbene analogues in solution. ${ }^{124}$ Even in some cases, they are called so and the idea is supported by presenting canonical forms (Figure 1.14). ${ }^{20,122,123}$ But other evidence like quantum chemical calculations suggest the presence of a single bond. ${ }^{123}$ All these discussions suggest that such interactions are non-classical double or triple bonds where the $\pi$-electrons are converted to non-bonding lone pairs and tend to localize at the metal atoms. ${ }^{121}$

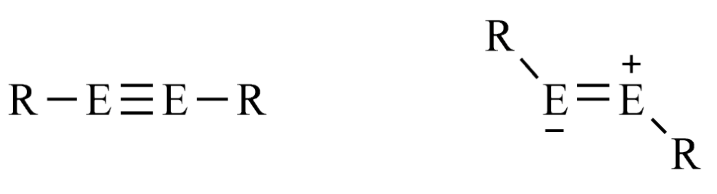

(A)
(B)<smiles>[R][P-]P[Fe]</smiles>

(C)

Figure 1.14: Canonical forms for alkyne analogues where $\mathrm{E}=\mathrm{Ge}, \mathrm{Sn}$ and $\mathrm{Pb}$

Similarly, the Group 13 and 15 heavier metal complexes can be considered to analyze the $\mathrm{M}-\mathrm{M}$ interactions in them. The highly controversial Robinson's red salt ${ }^{125}$ was suggested to contain a Ga-Ga triple bond based on experimental bond length of 2.32 $\AA$ which is well below the double of its covalent radius i.e. $2.44 \AA .{ }^{126}$ The argument was supported by different theoretical population analysis methods, ${ }^{127}$ while Bader's atoms-in-molecules method suggested a Ga-Ga single bond. ${ }^{128}$ Group 15 metals have conventional doubly bonded $\mathrm{M}=\mathrm{M}$ interactions ${ }^{129}$ in complexes due to the overlap of $p$ orbitals and the presence of lone pairs enriched in $s$ character. This discussion proves the diversity of bonding properties of main-group metal complexes where computational or theoretical power also helps a lot to validate the results. 
In addition, the nature of a metal-metal bond based on the amount of electron density can also be decided whether a close-shell (including donor-acceptor and ionic) or an open-shell (shared electrons such as in covalent bonds) through quantum mechanics. ${ }^{130}$ Furthermore, the energy of an interaction can be decomposed into various energy terms that can help to analyze the different types of repulsive and attractive interactions such as Pauli's repulsion, electrostatic and orbital attractions. ${ }^{131}$ We are interested in using different computational techniques to study the nature of bonds and coordination in main-group metal compounds.

\subsubsection{Reactivity}

Main-group metal compounds are well-known to undergo different types of reactions. ${ }^{132-135}$ As evident from an overview in the previous sections, these compounds have interesting structural features and bonding properties which force towards further exploration of their reactivity. Hetero-bimetallic compounds of main-group metals also possess a versatile reactivity with potential applications in catalysis, materials, and the synthesis of new reagents. ${ }^{136,137}$ Some complexes of gallium and indium with nickel are known to exist. Recently, Cammarota et al. reported the synthesis of some catalytically efficient main-group metal complexes with nickel (Figure 1.15). ${ }^{138}$ The heterobimetallic part was prepared through a two-step metalation process where the trihydride $\left(\mathrm{LH}_{3}\right)$ ligand is eventually converted to $\mathrm{Ni}-\mathrm{M}-\mathrm{L}$. $\mathrm{M}^{3+}$ imposes significant changes on the geometry on the structures of the complexes. Larger ions prefer to reside above the $\mathrm{N}_{3}$-plane and nickel moves higher above the $\mathrm{P}_{3}$-plane with the increase in size of these ions down the group even though normally $\mathrm{Ni}$ tends to be coplanar with - $\mathrm{PiPr}_{2}$ electron-donating moieties.

The synthesis of a complex with a chlorobismuthine ligand in coordination with [AuCl(tht)] (tht $=$ tetrahydrothiophene) was reported ${ }^{139}$ as shown in Scheme 1.4 where Au is a donor and $\mathrm{Bi}$ is acceptor. One of the products i.e. (27) is a dinuclear complex that has been 


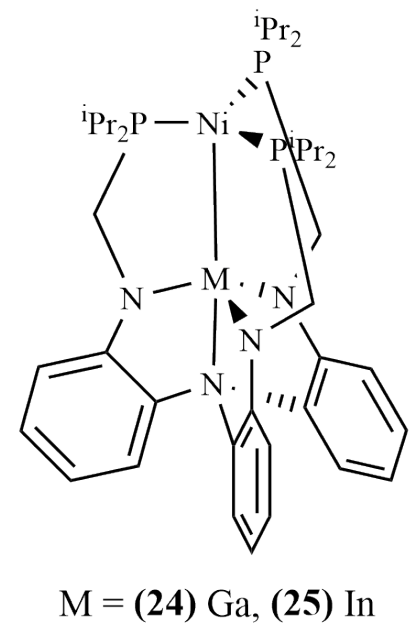

Figure 1.15: Heterobimetllic complexes of $\mathrm{Ga}$ and $\mathrm{In}$ containing Ni-M interactions (where $\mathrm{M}=\mathrm{Ga}$ and $\mathrm{In}$ )

afforded by the displacement reaction of (26) with $\mathrm{AuCl}$, in which $\mathrm{Cl}^{-}$was replaced by a phenylphosphino group. Hence, (26) may be regarded as a reagent in organometallic synthesis. The coordination pattern in (28) can be best analyzed based on its reported X-ray crystallographic data. Au exhibits a distorted square planar geometry. This is the characteristic of its trivalent state while $\mathrm{Bi}$ is in sphenoid form instead of a tetrahedral geometry. These factors collectively suggest the presence of a $\mathrm{Au} \rightarrow \mathrm{Bi}$ Lewis acid-base pair coordination. However, the Group 15 metal in this case and some other related cases were reported as trivalent centers and not pentavalent ones. ${ }^{140}$<smiles>Pc1ccccc1-c1ccccc1[BH-](c1ccccc1)c1ccccc1</smiles>

(26)

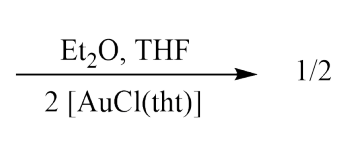

$1 / 2$

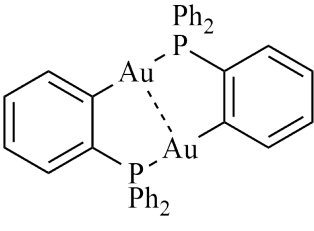

(27)<smiles>Cl[Te]1(Cl)[Pb]2Pc3ccccc3[Pb]21c1ccccc1</smiles>

(28)<smiles>[PbH2]=Cc1ccccc1</smiles>

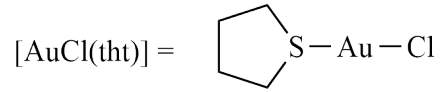

tht $=$ tetrahydrothiophene

Scheme 1.4: Synthesis of a $\mathrm{Au} \rightarrow \mathrm{Bi}$ complex. Reprinted with permission (Licence number: 4694270339676) from reference ${ }^{139}$ Copyright (2012) John Wiley and Sons.

The coordination of chlorobismuthine was also investigated (Scheme 1.5) with phenylphosphinopalladium and the former again showed the $\sigma$ accepting properties. ${ }^{139} \mathrm{Com}-$ 


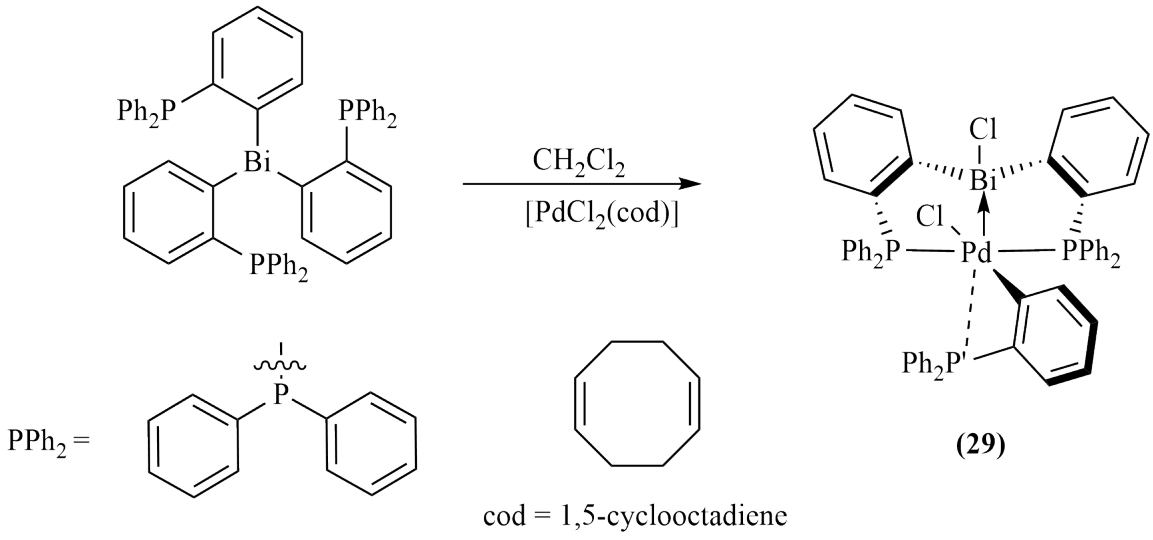

Scheme 1.5: Coordination of a chlorobismuthine with a phenylphosphinopalladium chloride. Reprinted with permission (Licence number: 44694270721888) from reference ${ }^{139}$ Copyright (2012) John Wiley and Sons .

plex (29) shows that $\mathrm{Bi}$ lies directly over $\mathrm{Pd}$ and an additional $\mathrm{Pd}-\mathrm{PPh}_{2}$ interaction was suggested as compared to (28). The coordination geometry of palladium in this case can be suitably regarded as a distorted octahedron. Further electronic information about (28) and (29) was gained from computational means. ${ }^{139}$ The optimized geometries closely resembled the experimentally determined crystal structures. However, the $\mathrm{Cl}$ interaction with $\mathrm{Bi}$ in both cases was suggested to be a dative bond where the lone pair of $\mathrm{Cl}$ interacts with an empty $p$ orbital of $\mathrm{Bi}$. The same orbital of $\mathrm{Bi}$ was shown to be responsible for the interaction with one of the filled $d$ orbitals of the Au and Pd atoms.

Previously, Jones et al. reported heterobimetallic compounds containing Ga-M ( $\mathrm{M}=$ $\mathrm{Na}, \mathrm{Zn}, \mathrm{Cd}$ ) bonds ${ }^{141}$ as shown in Scheme 1.6. The NHC analogue of $\mathrm{Ga}$ in (30), (31), (32), (33) and (34) behaves as donor to the attached metals.

Extending the above concept, the Coles' group at Victoria University of Wellington is investigating the synthesis and reactivity of indium-containing heterobimetallic compounds. We are interested in the nature of bonding in these compounds that will also be the subject of our investigations. 

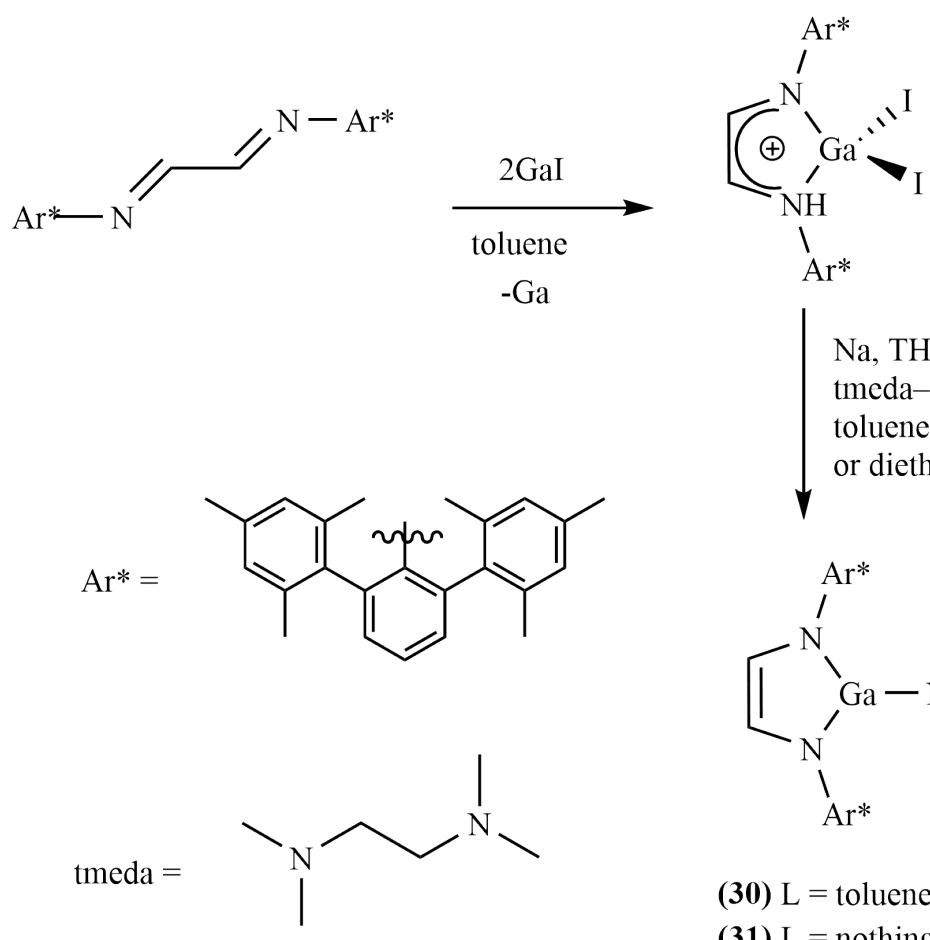

$$
\begin{aligned}
& \mathrm{Na}, \mathrm{THF},-\mathrm{NaI} \text {; } \\
& \text { tmeda-diethyl ether; } \\
& \text { toluene, toluene--hexane } \\
& \text { or diethyl ether }
\end{aligned}
$$<smiles>[Al+2]N1C=CN([Al+2])[GeH2]1</smiles>

(30) $\mathrm{L}=$ toluene

(31) $\mathrm{L}=$ nothing (32) $\mathrm{L}=\mathrm{OEt}_{2}$

$$
\text { (30) } \underset{\left[\mathrm{CdI}_{2} \text { (tmeda) }\right],-\mathrm{NaX}}{\stackrel{\left[\mathrm{ZnBr}_{2} \text { (tmeda) }\right] \text { or }}{\longrightarrow}}
$$

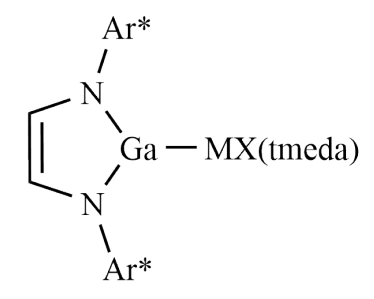

(33) $\mathrm{M}=\mathrm{Zn}, \mathrm{X}=\mathrm{Br}$

(34) $\mathrm{M}=\mathrm{Cd}, \mathrm{X}=\mathrm{I}$

Scheme 1.6: Heterobimetallic compounds with Ga-M bonds. Reprinted with permission (Licence number: 1000314-1) from reference ${ }^{141}$. Copyright (2012) Royal Society of Chemistry.

\subsection{Benzanthrone-Based Fluorescent Dyes}

Benzanthrone (Figure 1.16) is a polyaromatic ketone forming dyes that are organic luminophores. They fluoresce anywhere in the green to red region of the electromagnetic spectrum. ${ }^{142}$ Intramolecular donor-acceptor interactions have long been known to exist in molecules with fluorescence characteristics. ${ }^{9}$ Moreover, extended $\pi$ conjugation such as the one present in dyes inserts photophysical and photochemical properties ${ }^{143-145}$ which are subsequently responsible for the luminescence ${ }^{146,147}$ and fluorescence $^{148-150}$ characteristics of these compounds. 


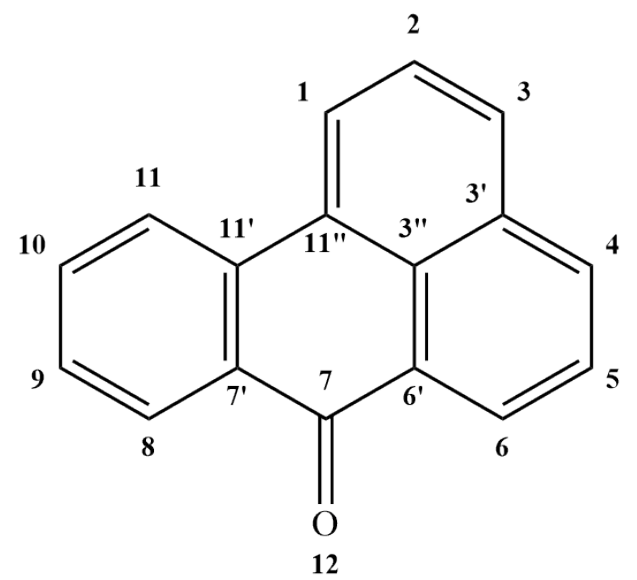

Figure 1.16: Benzanthrone

The recent increased interest in the synthesis of 3-aminobenzanthrone dyes is attributed to the fact that they are potential candidates in manufacturing liquid-crystalline material and opto-electronic devices. ${ }^{151-156}$ Compounds of benzanthrone other than 3-N-based dyes are also known such as 3-oxy derivatives, ${ }^{155,157}$ however, the most notable are the former. ${ }^{150,158-160}$ Gonta et al. reported a series of 3-amidobenzanthrone derivatives (Scheme 1.7) with absorption in the range 410-490 $\mathrm{nm}$ and emission at 505-665 $\mathrm{nm}$ which is significantly high Stokes' shifted fluorescence. ${ }^{149}$ Computational investigation was reported to suggest that ' $R$ ' groups do not have a significant impact on the charge of the neighbouring carbon. However, the dipole moment is affected significantly with changing $\mathrm{R}$ substituents.<smiles>Nc1ccc2c3c(cccc13)C(=O)c1ccccc1-2</smiles>

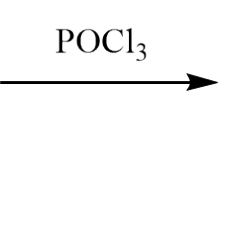<smiles>[R]N([R2])c1ccc2c3c(cccc13)C(=O)c1ccccc1-2</smiles>

$$
\begin{aligned}
& \text { (35) } R=M e, R_{1}=H, R_{2}=P h \\
& \text { (36) } R=H, R_{1}=E t, R_{2}=E t \\
& \text { (37) } R=M e, R_{1}=H, R_{2}=M e C_{6} H_{4}
\end{aligned}
$$

Scheme 1.7: Fluorescent amidobenzanthrone dyes. Reprinted with permission (Licence number: 4702151245259) from reference ${ }^{149}$. Copyright (2012) Elsevier B. V. 
A number of benzanthrone dyes undergo polymerization. ${ }^{161-163} \mathrm{Xu}$ et al. utilized the process of electrochemical polymerization and reported the synthesis of a semi-conducting polybenzanthrone ${ }^{162}$ with interesting green-light fluorescence. They proposed the mechanism of this electropolymerization as shown in Scheme 1.8. Cyclic voltametry in $\mathrm{CH}_{3} \mathrm{CN}$ was employed to monitor this reaction and it was reported that benzanthrone electropolymerization was made possible by enhancing redox wave current. Computationally-calculated atomic electron density populations ${ }^{162}$ were reported to show that $\mathrm{C} 1, \mathrm{C} 3, \mathrm{C} 4, \mathrm{C} 6, \mathrm{C} 8$ and $\mathrm{C} 11$ bear negative charges that can trigger the formation of radical cations to undergo electrochemical polymerization.

In another study by Konstantinova et al., co-polymerization of benzanthrone dyes with methylmethacrylate was reported. ${ }^{164}$ It was reported that over $50 \%$ of the concentration of the benzanthrone dyes co-polymerized and the copolymers thus produced had an enhanced photostability compared to the corresponding dyes.

Being efficient fluorophores, benzanthrone dyes are also potential candidates to form fluorescent probes for biomolecules ${ }^{152}$ and membranes. ${ }^{151}$ Ryzhova and co-workers reported the synthesis of such interesting dyes with potential applications in fluorescencebased membrane and protein studies. ${ }^{165}$ In another study, Kirilova and co-workers reported the synthesis of 3-aminobenzanthrone dyes and were also able to crystallize one of them (38) shown in Figure $1.17^{166}$ Although the substitution at position-3 of benzanthrone is more frequently reported, there are examples with different substitution sites on benzanthrone nucleus. ${ }^{167,168}$ Debeaux et al. reported the synthesis of an enol at position-7 along with the substitution of a diphenyl at position- 6 of the benzanthrone framework (Figure 1.18). ${ }^{167}$ Earlier, displacement of different substituents on position6 of the benzanthrone framework (the then called meso-benzanthrone) was proposed by Bradley. ${ }^{169}$ The nucleophilic substitution results in the electron-flow from the electrondonating substituents through the benzanthrone framework to the carbonyl group gives rise to the donor-acceptor interactions, thus imparting intramolecular charge transfer which can be spectroscopically exploited. 


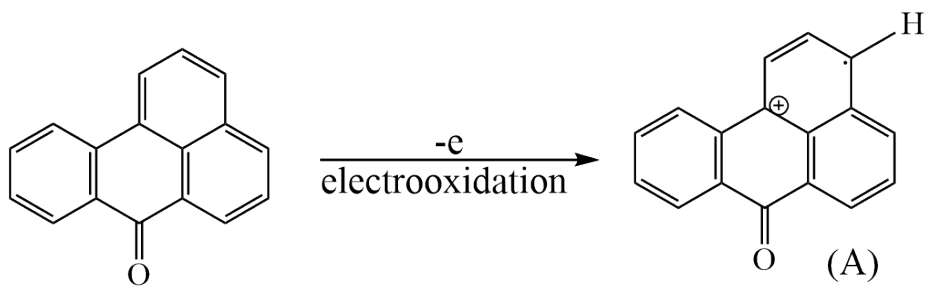

or<smiles>O=C1c2ccccc2-c2cccc3cccc1c23</smiles><smiles>CC1C=CC2c3ccccc3C(=O)c3cccc(c32)C1</smiles>

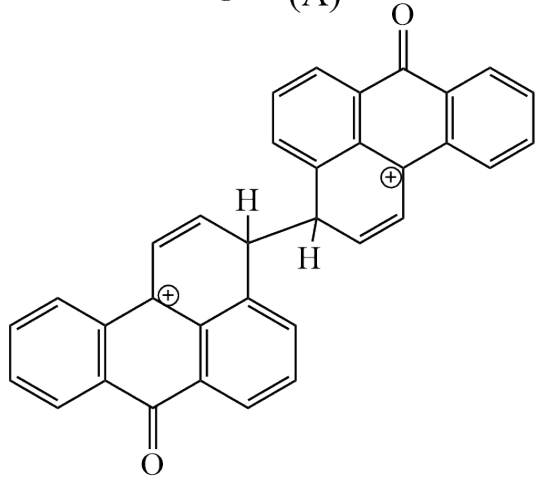<smiles></smiles><smiles></smiles>
coupling with B<smiles>C=C1c2ccccc2C(=O)c2cccc(C)c21</smiles>

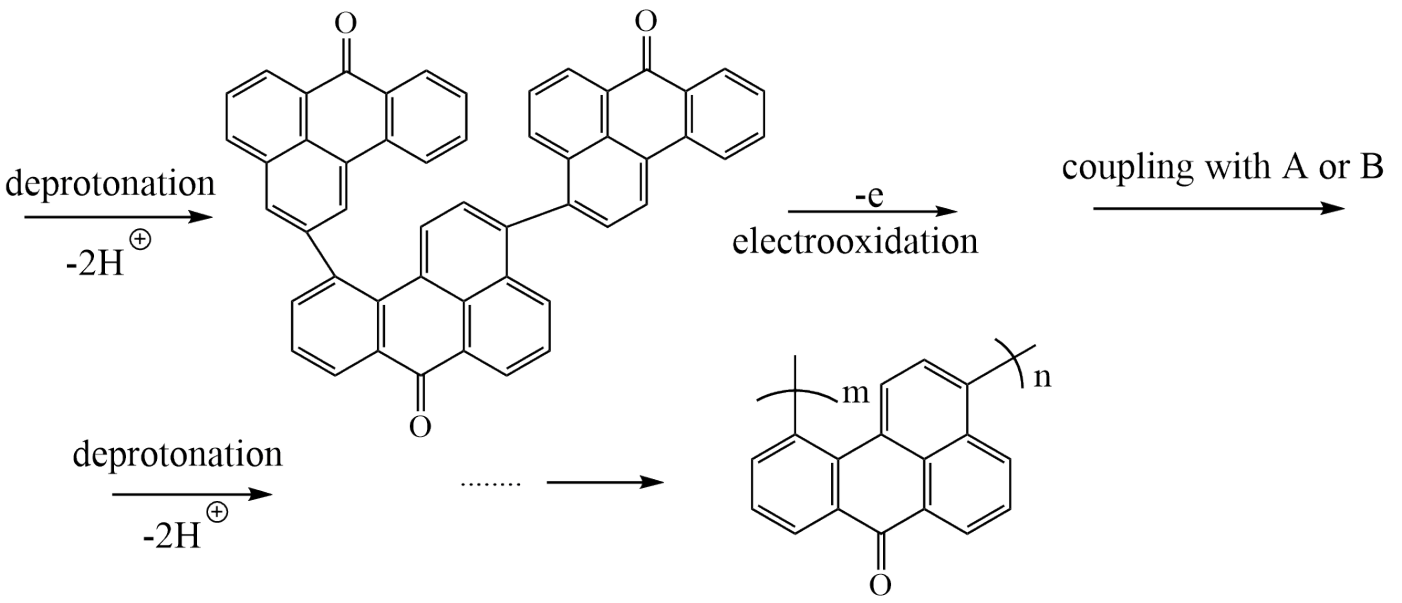

Scheme 1.8: Mechanism of electropolymerization of benzanthrone. Reprinted with permission from reference ${ }^{162}$. Copyright (2009) American Chemical Society.

To further enhance their applications in materials requiring fluorescent precursors, photophysical and photochemical parameters of the benzantheone dyes must be explored. Few efforts have already been made in this regard. For instance, Kapusta et al. investigated the effect of changing solvent on intersystem crossing. ${ }^{170}$ With the increas- 


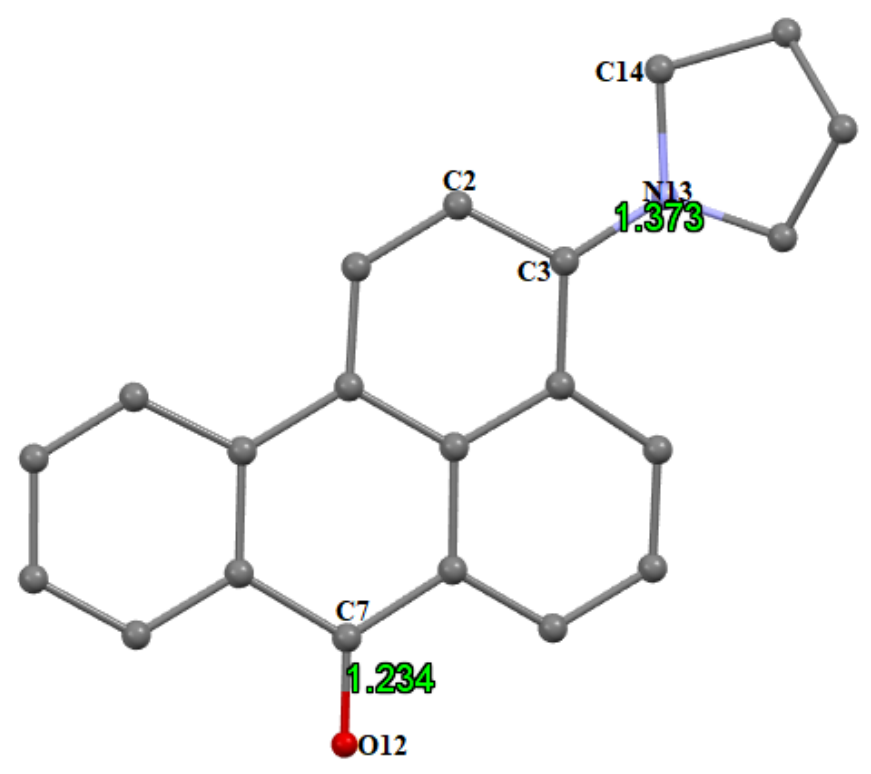

(38)

Figure 1.17: Crystal structure of a 3-aminobenzanthrone dye (38) reported by Kirilova et al. ${ }^{166}$ Hydrogen atoms omitted for clarity. Reprinted with permission under the copyright licence number: 4694951097894 from the publisher. Copyright (2009) Elsevier B.V.

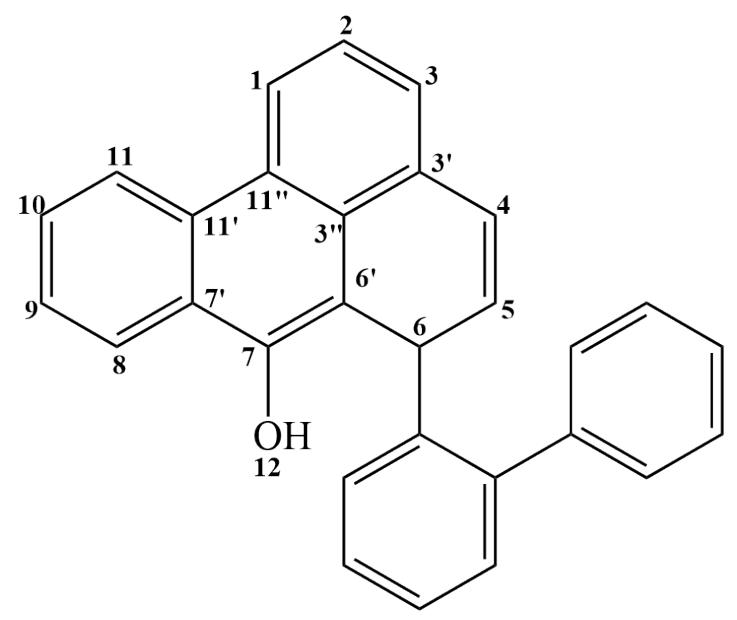

Figure 1.18: Synthesis of an enol at $\mathrm{C}=\mathrm{O}$ in benzanthrone framework along with the substitution of a diphenyl at postion- 6 of benzanthrone framework.

ing polarity of the solvents, the singlet to the corresponding triplet state energy gaps increase significantly. This shows that non-radiative emission is not facilitated in increasingly polar solvents. Photophysics of benzanthrone dyes was also explored in another study on the basis of HOMO and LUMO energies and the dipole moments. ${ }^{160}$ However, conclusive predictions in this case remained impossible likely because of the 
over-estimation of dipole moments due to low computational effort.

A detailed insight is deemed necessary to get an enhanced understanding of the photophysics involved in the fluorescence behaviour of 3-aminobenzanthrone dyes. For this purpose, the properties of the excited states of these compounds are needed to explore which has already been possible by the use of computational chemistry time-dependentDFT (TD-DFT) for other systems. ${ }^{171}$ To the best of our knowledge any detailed analysis of the excited states of the dyes under discussion is missing from the literature. The current contribution is an effort to this point to gain a deep understanding of the absorption and emission mechanism of 3-aminobenzanthrone dyes. For this purpose, the previously synthesized dyes ${ }^{172}$ reportedly possessing excellent fluorescence spectra were chosen.

\subsection{Aim of the Thesis}

As discussed in detail, compounds with donor-acceptor interactions find potential applications for metal chelation, ${ }^{39}$ catalysis ${ }^{22,173}$ and various types of chemical transformations. ${ }^{23-25}$ In the case of main-group metal cyclophane complexes, their applications are mainly based on their capacity for $\pi$-donation to metals. Heterobimetallic compounds of main-group metals having M-M' bonds may be tested for their reactivity. The applications of benzanthrone dyes are also based on donor-acceptor interactions that are different to the interactions in host-guest complexes. These dyes have always been suspected to undergo intramolecular charge transfer in their excited states that imparts fluorescence characteristics to these molecules. Such behaviour further makes them potentially useful for light-harvesting systems. However, despite the expectations based on their structures, benzanthrone dyes lack the experimental or computational efforts on an extensive scale to make effective use of these chemical species possible.

The current compuational study is dedicated to three different types of donor-acceptor 
systems with the following aims and objectives:

1. Insight into the structural and bonding properties of host-guest complexes of main-group metals with cyclophane ligands.

2. Investigation of the nature of indium-zinc bond in a heterobimetallic complex and the In-N and $\mathrm{Zn}-\mathrm{N}$ bonds in the product of that heterobimetallic compound as a result of mesityl insertion across the In-Zn bond.

3. Understanding the fluorescence mechanism in benzanthrone dyes through the study of electronic excitations. 


\section{Chapter 2}

\section{Computational Details}

Computational chemistry refers to the solution of chemical problems through computers using a mathematical approach in combination with the physical laws. It can provide useful information about chemical systems that may contain thousands of particles depending on the required level of accuracy. ${ }^{174}$ Computational chemistry may be based on classical mechanics or quantum mechanics depending on the mass and velocity of the system of interest. Classical mechanics is designated to obtain the exact solution to the problems related to macro systems such as atoms and molecules. The physical observables related to the sub-atomic particles such as electrons, on the other hand, require probabilistic interpretation that can be performed in quantum mechanics by solving time-independent Schrödinger wave equation (Eq. 2.1) for a system.

$$
\hat{H} \Psi=E \Psi
$$

where $\hat{H}$ denotes the Hamiltonian operator, $\Psi$ shows the wavefunction attached to the system and $E$ is the energy of that system. $\hat{H}$, as the sum of kinetic and potential operators, is given by 


$$
\hat{H}_{\text {Schrödinger }}=\hat{T}+\hat{V}
$$

The solution of the Schrödinger wave equation involves the Born-Oppenheimer approximation that treats nuclear and electronic motions as separate. ${ }^{175}$ In this way, the positions of the nuclei are considered as parameters while solving the electronic component. The potential energy surface obtained as a result of this solution provides the basis to further solve the nuclear part. ${ }^{174}$ However, the exact solution using computational methods is limited to only smaller systems (involving one or two particles). When it comes to more complicated systems, the solution can only be approximated using these methods which can be further refined to higher accuracy at higher computational cost.

The solution of chemical systems with more than two electrons requires a relatively complex computational approach such as the Hartree-Fock approximation which takes into account an approximated average electronic interaction of the whole system rather than individual interaction of an electron. The motion of each electron differs slightly from the others with respect to the overall potential field of the system. ${ }^{176}$ There is an orbital associated with each electron and the antisymmetrized and normalized product of all the orbitals yields the total wavefunction associated with the system. ${ }^{174}$ The probability of finding an electron is determined from the shape of the corresponding molecular orbital based on the attraction to the complete nuclear framework and average repulsion from all the electrons. Each orbital needs a separate solution, giving rise to the individual Hartree-Fock equations which in turn require an iterative solution. These equations may lead to the derivation of semi-empirical methods-through additional approximations not taking into account the integrals with more than two nuclei involved in the formation of the Fock matrix-or to convergence towards the exact solution of the Schrödinger wave equation by adding additional determinants. ${ }^{174,177}$

The accuracy of the Hartree-Fock approach is limited because the electron-electron correlations are neglected and only an average electronic interaction is taken into account. 
The difference between the energy thus calculated and the lowest possible computable energy is called the electron correlation energy of the system any given basis set. In most cases, this difference is significant to explain the chemical systems as this small fraction of neglected energy may cause deviations large deviations from the actual results. ${ }^{174}$ Electron correlation can be calculated through various methods such as coupled cluster, many-body perturbation theory and configuration interaction (CI). However, for the bigger systems, these methods incur high computational cost. A different approach to solving the computational problems, called density functional theory (DFT), was then proposed based on electron density. Since the current computational study has been carried out using DFT, some details will be discussed in this chapter.

\subsection{Basic Concepts of Density Functional Theory}

DFT is based on the Hohenberg-Kohn variational theorem which states that the electronic ground state density can completely define the associated potential. ${ }^{178}$ Mathematically, this can be expressed as

$$
E_{v(r)}\left[n^{\prime}(r)\right]=\int v(r) n^{\prime}(r) d r+F\left[n^{\prime}\right]
$$

Here, all the ground state non-degenerate densities $n^{\prime}(r)$ have a functional $F\left[n^{\prime}(r)\right]$ and $E$ shows the minimum value of ground-state energy associated with external potential $v(r) . F\left[n^{\prime}(r)\right]$ is further given by

$$
F\left[n^{\prime}(r)\right]=\left(\Psi_{n \prime(r)},(U+T) \Psi_{n^{\prime}(r)}\right)
$$

where $\Psi_{n \prime(r)}$ denotes the ground state with the density $n^{\prime}(r)$. The operators $\mathrm{T}$ and $\mathrm{U}$ belong to the Coulomb repulsion and kinetic energy respectively.

This treatment of the electronic part of a chemical system has preference over the 
wavefunction-based treatment. There are one spin and three spatial coordinates (variables) associated with each electron in the wavefunction approach and hence the complexity increases with the increasing number of electrons. In calculating the electronic energy as a function of electron density, the number of variables is lowered as only three spatial coordinates are required to explain each spin density. However, the limitation still remains that the functional connecting the energy and the electron density is not known. These functionals are an integral part of DFT. It is noteworthy at this point that a functional is the way to generate a number from a function which is itself the way to obtain a value from a set of coordinates. ${ }^{174}$ Energy in this case as a functional depends on the functions viz the electron density and the wave function. Designing earlier DFT functionals were based on taking into account a uniform electron gas. The Thomas-Fermi model divides the energy functional $\left(E_{T F}[\rho]\right)$ as

$$
E_{T F}[\rho]=T_{T F}[\rho]+E_{n e}[\rho]+J[\rho]
$$

where $T_{T F}[\rho]$ represents the kinetic energy, $E_{n e}[\rho]$ shows the attraction between the nucleus and electrons and $J[\rho]$ denotes the classical Coulomb repulsion. Bloch and Dirac added the exchange term $K[\rho]$ in equation 2.5. Hence, the Thomas-Fermi-Dirac equation is given as

$$
E_{T F}[\rho]=T_{T F}[\rho]+E_{n e}[\rho]+K[\rho]+J[\rho]
$$

However, the Thomas-Fermi model that in fact uses the uniform electron gas model can only explain a few systems such as metals but since it does not consider bonding, it fails in atoms and molecules. ${ }^{174}$

An improved model of DFT was presented by Kohn and Sham putting forward an indirect approach toward the kinetic energy functional $T[\rho] .{ }^{179}$ According to this approach, the kinetic energy can be solved to a good accuracy while the residual correction is 
treated separately. ${ }^{180}$ For a system with a more generalized potential i.e. the one with a usual scalar potential accompanied by a magnetic field, the spin-electron density also needs to be taken into account in addition to the total electron density. In this way, the theory may be referred to as spin-density-functional-theory. The exact energy $\left(T_{s}\right)$ is calculated, by considering that electrons are non-interacting. However, electrons are interacting in reality giving rise to the smaller fragment of kinetic energy called the exchange-correlation term $E_{x c}$. The energy functional $\left(E_{D F T}[\rho]\right)$ according to this model can thus be given as

$$
E_{D F T}[\rho]=T_{s}[\rho]+E_{n e}[\rho]+E_{x c}[\rho]+J[\rho]
$$

The exchange-correlation energy functional in the above equation $\left(E_{x c}[\rho]\right)$ can be further split as

$$
E_{x c}[\rho]=\left(T[\rho]-T_{s}[\rho]\right)+\left(E_{e e}[\rho]-J[\rho]\right.
$$

Here, $T[\rho]-T_{s}[\rho]$ explains the kinetic correlation term and the latter parentheses contain potential exchange and potential correlation energy respectively. Below is a brief account of various methods to calculate the exchange-correlation energy.

\subsubsection{DFT Methods Dealing with Exchange-Correlation Energy}

In early attempts toward the solution to the exchange-correlation energy, both the exchange and correlation functionals were preferably treated separately and they were combined later. However, current emphasis within the computational science community is to study the two fragments in a combined manner because only the combined correlation and exchange terms provide physical meaning. Below is a brief introduction to some of the significant methods dealing the exchange-correlation energy. 


\subsubsection{Local density approximation (LDA)}

Classification of the electronic regions with respect to the electron density in the KS model as mentioned above shows that the density near the nucleus tends to remain uniform and next to it starts the region with slowly varying density. Practically following this assumption, LDA locally treats the electron density as a uniform electron gas or otherwise as a slowly varying function. Going back to the exchange-correlation energy, it is the sum of the correlation between the electrons of the same spin $(\alpha)$ and the correlation between the electrons of opposite spin $(\beta)$. Now, if the $\alpha$ and $\beta$ spin densities are different, a modified local spin density approximation (LSDA) applies. LDA and LSDA are equivalent for a closed shell. Regarding its efficiency to fully explain a system, LSDA proves successful where the density slowly varies such as metals. However, this method performs poor for molecular systems as it underestimates the exchange energy by approximately $10 \%$ thereby producing large errors. ${ }^{174}$ Bond energy may be overestimated by about $100 \mathrm{~kJ} / \mathrm{mol}$.

\subsubsection{Generalized Gradient Approximation (GGA)}

LSDA may be made to work better by considering the electron density as a non-uniform gas. This is possible by considering the exchange and correlation energies as functions of electron density as well as its first derivative. This gives rise to the GGA approach in which the first-order corrections of exchange and correlation energies are added to LSDA. Additionally in this method, the Fermi (exchange) and Coulomb (correlation) probabilities of electrons integrate to -1 and 0 respectively to make the model successful otherwise the simple addition of the above mentioned corrections would produce even worse results. A number of groups devised the GGA functionals including B88 ${ }^{181}$ (proposed by Becke for exchange energy correction), LYP ${ }^{182}$ (designed by Lee, Yang and Parr), PW86 ${ }^{183}$ (proposed by Perdew and Wang in 1986) and PW91 ${ }^{184}$ (proposed by Perdew and Wang in 1986). The GGA version by Perdew's group continued to 
improve and they contributed to give a new functional $\mathrm{PBE}^{185}$ (after the names Perdew, Burke and Ernzerhof).

\subsubsection{Meta-GGA and Hyper-GGA Methods}

Meta-GGA methods are an improvement on GGA wherein the exchange and correlation functionals are the functions of higher order derivatives of electron density such as the second order term Laplacian $\left(\nabla^{2} \rho\right)$ associated with the density. The orbital kinetic energy density and the Laplacian are similar with respect to the information they contain. Hence, either of these may be added to GGA to give rise to meta-GGA. Even more improvement is possible by combining gradient correction and LSDA along with the addition of an exact exchange energy term. This gives rise to the hyper-GGA, also called the hybrid methods. For instance, the meta-GGA functional PBE was improved to the hybrid PBE1PBE functional (usually known with the common name PBE0) by adding an exact exchange. ${ }^{186}$ Others include B3LYP (Becke, Lee, Yang and Parr) hybrid functional where the B88 ${ }^{181}$ exchange functional is combined with the LYP ${ }^{182,187}$ correlation functional. Improved results have been reported by including a suitable fraction of exact Hartree-Fock exchange, which is now a standard feature. ${ }^{174}$

DFT methods have some limitations as well. For instance, they do not count for the van der Waals dispersion forces arising in wave function models as a result of electron correlation. This results in the poor performance of the DFT functionals because of the incorrect $\mathrm{R}^{-6}$ limiting factor. To cope with this Grimme introduced a series of dispersion corrections ${ }^{188-190}$ with the latest being the D3 ${ }^{190}$ correction. Adding the D3 correction with Becke-Johnson (BJ) damping ${ }^{191}$ improves results as this is a model to make corrections regarding the weak dipersion forces. Hybrid functionals usually perform better than the GGA-type functionals. Kang et al. ${ }^{192}$ carried out a study to assess various DFT methods whereby keeping high quality ab initio coupled cluster CCSD(T) as reference. This was accompanied by a limit extrapolation of the complete basis set to assess various DFT methods. They analyzed molecular properties such as structural fea- 
tures and bond energies to draw a comparison of the DFT methods. It was reported that a single-hybrid PBE0 and the two double-hybrid functionals B2PLYP and B2GPLYP performed the best. Interestingly, adding the D3 correction improve the results obtained from B2PLYP to a greater extent while the B2GPLYP double-hybrid was inferior. However, a small but positive effect on PBE0 was noted when the D3-BJ correction was added. In another study reported by Grimme et al., PBE0-D3 functional performed even better than the double-hybrid functionals such as PWPB95-D3. ${ }^{193}$ It can be seen in the chart in Figure 2.1 that the mean absolute deviation against a $\operatorname{CCSD}(\mathrm{T})$ reference for this hybrid functional is the minimum i.e. $1.1 \mathrm{kcal} \mathrm{mol}^{-1}$ out of all the 24 functionals under the-then consideration of the authors.

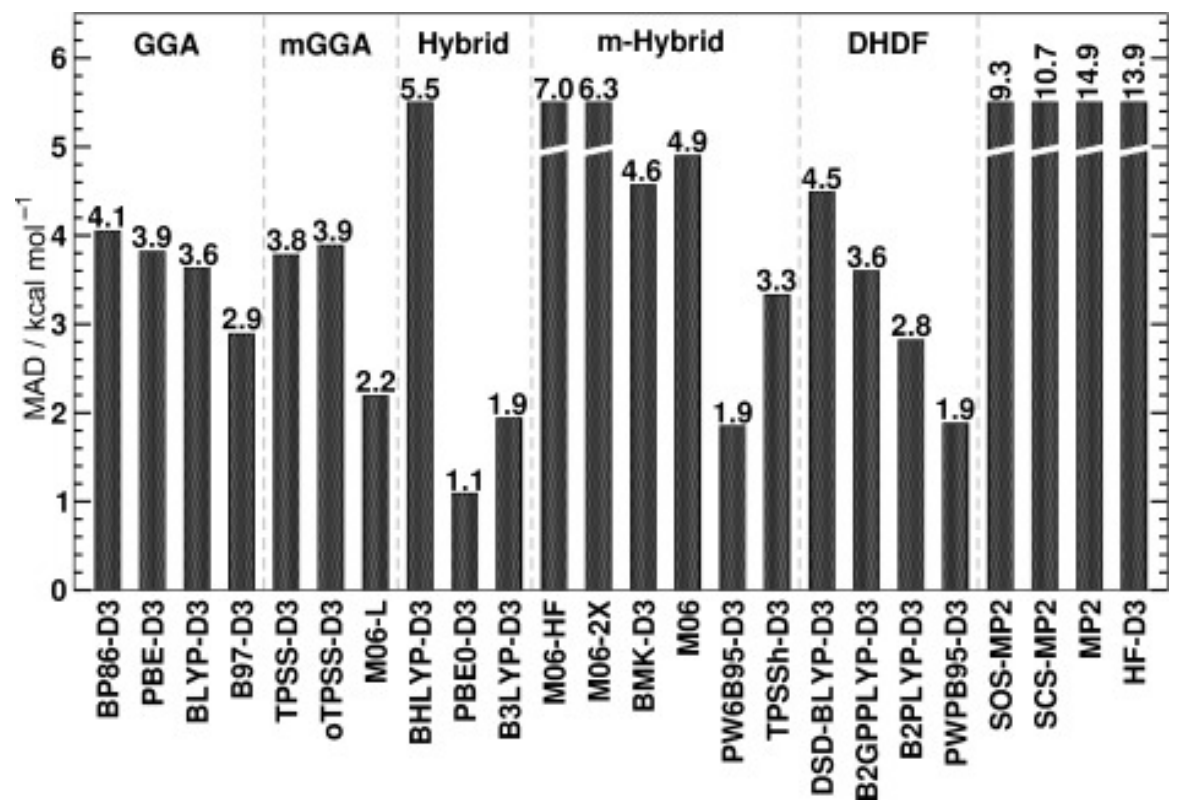

Figure 2.1: Mean absolute deviation (MAD) of different functionals from the reference CCSD(T) in the benchmark study by Grimme et al. ${ }^{193}$ Copyright (2013) Marc Steinmetz and Stefan Grimme. ChemistryOpen published by WILEY-VCH Verlag GmbH \& Co. KGaA, Weinheim.

\subsubsection{Basis Sets}

A basis set is a set of functions that can be used to generate algebraic equations from the partial differential equation in order to solve the unknown functions. In chemistryrelated problems, basis sets are employed in ab initio as well as DFT methods. Ideally 
an unknown function can be expanded over a set of already known functions. This, however, requires a complete basis set that in turn utilizes an infinite number of known functions and this is impossible in reality. In an infinite coordinate system, a molecular orbital may be referred to as an unknown function. Calculation using a finite basis will only calculate the part of the orbital corresponding to the selected basis set. Hence, the accuracy of the result depends on the quality of the basis functions present in the basis set used. This quality is represented in terms of the ability of a basis function to explain the unknown function. A higher number of these will be required if the basis functions are low-quality. However, this may increase the computational cost to a great extent as in $a b$ initio methods. Therefore, the selected basis set needs to be efficient in accuracy as well as computational cost.

Basis functions are of two types; Slater type orbitals (STOs) and Gaussian type orbitals (GTOs). ${ }^{174}$ STOs find application in atomic and diatomic calculations and are intended for high accuracy. These can also accompany the DFT methods that do not consider exact exchange. The functional expression of STOs given below:

$$
\chi_{\zeta, n, l, m}(r, \theta, \varphi)=N Y_{l, m}(\theta, \varphi) r^{n-1} e^{-\zeta r}
$$

In the above equation, $Y_{l m}$ is for spherical harmonic functions and $N$ denotes normalization constant. GTOs, on the other hand, are inferior to STOs with respect to their behaviour near the nucleus: the former has a zero slope on the nucleus while STOs make a cusp. Moreover, GTOs fall far from the nucleus and they are unable to represent the tail of the wavefunction efficiently. These limitations show that more GTOs are required than STOs to make a basis set. However, in terms of efficiency in calculating the integrals, GTOs work better and hence these are the basis functions that are now universally used for electronic calculations.

$$
\chi_{\zeta, l_{x}, l_{y}, l_{z}}(x, y, z)=N x^{l x} y^{l y} z^{l z} e^{-\zeta r^{2}}
$$


Here, $x, y, z$ shows the coordinates while the type of orbital is determined from the sum of $l_{x}, l_{y}$ and $l_{z}$ corresponding to the orbital angular momentum.

\subsubsection{Types of Basis Sets}

Basis sets can be classified according to the number of basis functions they have. The basis set with the smallest possible number of basis functions is called the minimum basis set, where there are only enough functions to count for all the electrons. This means for hydrogen only one $s$-function is needed while for sodium three $s$-functions and two $p$-functions are required. The second type basis set arises when basis functions double and is called double zeta basis set. The word zeta finds its origin analogous to the explanation of the exponent of STO basis that is denoted by the Greek letter zeta $(\zeta)$. The next improvement in the basis set gives rise to the triple $\zeta$ basis set wherein the number of basis functions becomes three times that in the minimum basis set. If some of the core orbitals are again saved by splitting the valence, the modified basis will be triple zeta split valence. In a similar fashion, the basis functions may be increased and the name will change accordingly to quadruple zeta, quintuple zeta and so on.

Polarization functions are also added in cases where higher angular momentum needs to be included. For instance, the $p$ orbital is added to account for the polarization of $s$ orbitals. Similarly $d$ and $f$ orbitals may be included to introduce polarization of $p$ and $d$ orbitals respectively. Electron-correlation methods essentially need polarization to be added. Correlation is the lowering of energy when electrons tend to keep far away from each other. It means the angular correlation arises when one of the two electrons is on either side of the nucleus, which is explained by polarization functions in a basis set. Adding a single set of polarization functions to a double zeta basis set gives rise to the modified double zeta polarization (DZP) basis set and doing the same to a split valence triple zeta basis set will change it to triple zeta valence polarization (TZVP) basis. Similarly, more than one set of polarization functions may be added. For example, addition of the two sets to a triple zeta basis set constitutes TZ2P basis. 
Based on the method of basis set optimization, basis sets are of two types; the ones with all-energy optimization (further divided in two types i.e. even and well-tempered basis sets) and the others with contracted number of functions (contracted basis sets). ${ }^{174}$

\subsubsection{Commonly Used Basis Sets}

Different research groups have designed basis sets to meet the needs of computational chemists. The focus is on contracted basis sets as contraction results in the reduction of computational cost in spite of the increase in energy. Few significant groups of basis sets are outlined below.

2.1.2.2.1 Pople Basis Sets One of the earliest group of basis sets with different qualities was developed by Pople and coworkers. ${ }^{194}$ The minimum basis set in this group is known as STO-3G. These slater type orbitals consist of three primitive GTOs. Although STO-4G to STO-6G are also known, such a basis set with the number of GTOs higher than three exhibits no significant improvement. Hence, STO-3G is widely used for various elements. Moving to a higher level with split valence is the basis set 3-21G which is a contraction of three primitive GTOs representing the core orbitals. The valence orbital is represented by two and one contracted orbitals for inner and outer part (of the orbital), respectively. In a similar fashion, higher split valence Pople basis sets include 6-31G and 6-311G where the first digit shows the number of contracted primitive GTOs representing core orbitals while the next two or three digits explain the splitting of valence orbital. Diffuse functions can also be added indicated by + for $s$-functions and ++ for $p$-function and are written before $\mathrm{G}$. If the polarization functions are to be added, they are denoted usually in parentheses after G. For example, a 6-31G after adding diffuse $p$-functions and a $d$ type polarization will be represented as $6-31++\mathrm{G}(\mathrm{d})$.

2.1.2.2.2 Ahlrichs Basis Sets Ahlrichs and co-workers have also designed the basis sets of varying qualities such as double zeta ${ }^{195}$ and triple zeta. ${ }^{196}$ These are the default 
basis sets used in TURBOMOLE software so they got the names starting with "def". However, it was observed that the accuracy is not consistent for many elements from

one system to the other. Therefore the Alrichs' group proposed the corrections ${ }^{197}$ to induce consistency and the new names start from "def2" such as def2SVP, def2TZVP, def2QZVPP and so on. These basis sets have been tested and found efficient for all the elements from $\mathrm{H}$ to $\mathrm{Rn}$.

\subsection{Size of a System and Accuracy}

The measurement of a system, i.e. small or large, is a relative term that changes based on the computational scheme used. The more the computational power used, the higher the accuracy will be at the cost of an increased computational time. ${ }^{174}$ For example, for a hybrid functional such as PBE0 (that is used throughout this study) along with a basis set of triple zeta quality in electronic structure methods, diatomic molecules such as $\mathrm{CO}$ and $\mathrm{N}_{2}$ are small systems; a compound with thirty to forty heavy atoms will be considered large and macromolecules such as proteins are too huge to handle using the electronic structure methods. In any particular computational method, an increase in the electron correlation and the number of subsequent basis functions to be calculated limits the size of the system that can be handled in that system. ${ }^{174}$ Hence, a chemical system that is very small for one method may be considerably large for another.

\subsection{Methodology in the Current Study}

The current study utilizes the functional PBE0 with D3-BJ dispersion correction. The usual representation PBE0-D3BJ/basis-set will be used to show the level of DFT at which a study was carried out, which exhibits the functional used along with the correction and the type of basis set written after the slash. 
The study of the coordination of cyclophanes with heavier main-group metals consists of two parts: (1) geometry optimization and vibrational analysis to analyze the structural properties and thermodynamics and (2) Morokuma-Ziegler energy decomposition analysis (MZEDA) to predict the strength of different bonding interactions in the complexes optimized in part (1). The first part was carried out in Gaussian 09 suit of programs at PBE0-D3BJ/def2TZVP level while Amsterdam Density Functional (ADF) package was used to perform MZEDA at PBE0-D3BJ/TZ2P level.

Fluorescence characteristics of benzanthrone dyes were studied in Gaussian 09 at PBE0D3BJ/def2TZVP level. Geometry optimization was followed by the calculations for vertical excitation and excited state optimization. Natural transition orbitals analysis was carried out on the vertically excited molecules to compute the map of difference of density that arises upon vertical excitation from the ground state. All the calculations were carried out both in the gas phase and the solvent phase to observe the behaviour of the dyes upon dissolution in a polar solvent such as ethanol. The SMD solvent model was added to count for the solvent effects.

The structural features and bonding properties examined through NBO analysis of main-group organometallic compounds as a part of collaborative work was performed in Gaussian 09 at PBE0-D3BJ/def2SVP level. The optimized geometries were further subject to calculate natural bond orbitals (NBOs).

Molecular graphics in all the cases were visualized in GaussView 5.0.9.

Below is a brief overview of some important terms used in methodology.

\subsubsection{Geometry Optimization}

Changes in the geometry of a compound induce changes in its energy which can be explained on the basis of potential energy surface (PES). PES is a relationship that joins the changes in the structure with the resultant changes. ${ }^{198}$ The dimensions of the plot of 
PES for a molecule increase with an increase in degree of freedom. The points on the PES may either be maxima or minima with respect to one another, thus creating valleys of different energy. The minimum is the lowest point in a valley. If this valley is the lowest in energy compared to the rest of the PES then the minimum would be called global minimum. However, if the minimum is only the lowest point with respect to a particular region then it is local minimum. A minimum is an equilibrium geometry in the case of multicomponent systems such as those having different conformations. Maxima are the peaks and ridges in a valley and these too may be local or global. A point between two maxima which is itself a maximum in the plane perpendicular to those maxima is called saddle point. The saddle point is in fact a transition structure between two equilibrium geometries. There is a preset criterion by Gaussian to which a structure must converge to be considered as a stationary point. Such a converged structure will have the value for forces and their root mean-square, and the maximum displacement from one step to the other and the root mean-square of the maximum displacement less than 0.00045 hartrees/bohr, 0.0003 hartrees/radian, 0.0018 and 0.0012 respectively. ${ }^{199}$ To confirm whether the obtained stationary point is an equilibrium structure or not, vibrational analysis is performed. Presence of any negative frequency, known as imaginary frequency, renders the structure a transition structure while if all the frequencies are positive then it is equilibrium geometry.

\subsubsection{Morokuma-Ziegler Energy Decomposition Analysis (MZEDA)}

MZEDA is a method to explain the chemical bonds in terms of different energy terms. ${ }^{131,200}$ It provides an estimation of the strength of interaction between two fragments of a molecule. ${ }^{201}$ It involves the decomposition of the bond dissociation energy $(\Delta \mathrm{E})$ of a compound $\mathrm{AB}$ as:

$$
\Delta E=\Delta E^{\mathrm{prep}}+\Delta E^{\mathrm{int}}
$$


Here, $\Delta E^{\text {prep }}$ is the energy required to prepare the two fragments $\mathrm{A}$ and $\mathrm{B}$ of the molecule $\mathrm{AB}$ to change their electronic state and structure from their equilibrium ground state geometry to the one in their parent molecule $\mathrm{AB} . \Delta E^{\mathrm{int}}$ is the instantaneous interaction energy between the two fragments in the molecule that can be further decomposed an in Equation 2.12:

$$
\Delta E^{\mathrm{int}}=\Delta E^{\mathrm{rep}}+\Delta E^{\mathrm{ele}}+E^{\mathrm{orb}}
$$

$\Delta E^{\text {rep }}$ is due to the repulsion arising among the same-spin electrons. $\Delta E^{\text {orb }}$ is the energy due to covalent interaction and can be further decomposed to the interaction of different orbitals with respect to the point group of the molecule. It is always an attractive energy term. $\Delta E^{\text {ele }}$ belongs to the electrostatic interaction between $\mathrm{A}$ and $\mathrm{B}$. $\Delta E^{\text {ele }}$ and $\Delta E^{\text {rep }}$ may cancel out each other which can lead to the incorrect assumption that only orbital interactions are present. Moreover, the sum of both $\left(\Delta E^{\mathrm{ele}}\right.$ and $\left.\Delta E^{\mathrm{rep}}\right)$ may result in an attractive term through which one can think that the Pauli repulsion is absent. To avoid these misconceptions, separate explanation of the terms is advised. ${ }^{202}$

\subsubsection{Excited States}

Any specific probability distribution explaining the electron density is referred to as an electronic state. However, the laws of physics suggest that only few of these can exist for a particular system. ${ }^{199}$ Of these allowed states, the one with the lowest energy is the ground state and all the other states are called excited states. A molecule can go into one of these states by absorbing energy where it usually stays for a short period of time and then relaxes back to its ground state as a result of de-excitation. One way to relax to the ground state is by emitting a longer wavelength of energy than the one absorbed.

Figure 2.2 shows the excitation and emission phenomenon of a molecule. It can be seen that there are various possible vibrational levels on the potential energy surface 


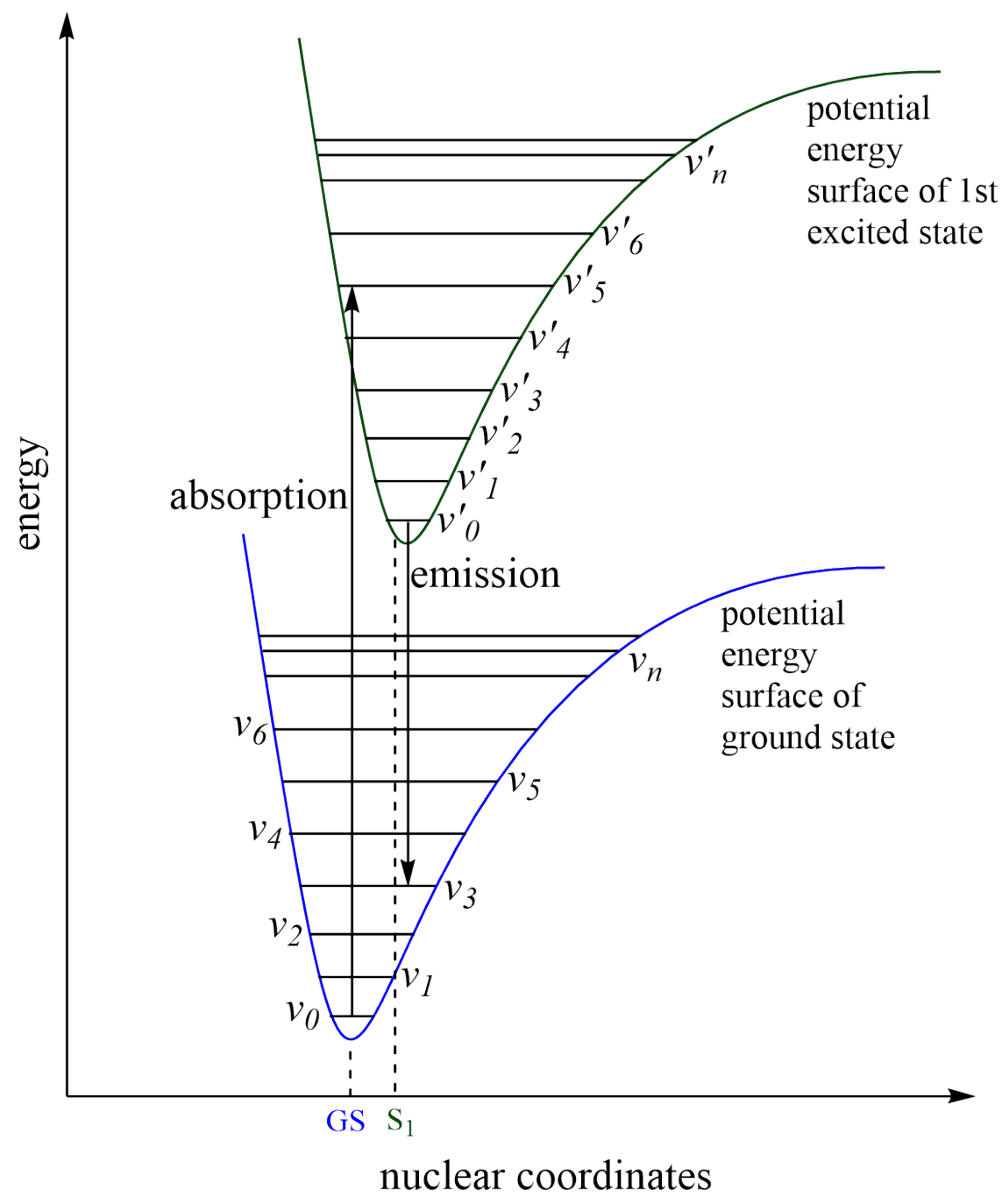

Figure 2.2: Mechanism of a molecule going to the first excited state from ground state

of an energy state. The absorption by the molecule causes it to reach the fifth vibrational level of the first excited state from vibrational ground state. Also, when it emits energy, it goes into the third vibrational level of of the ground state. This mechanism of electronic transitions (both absorption and emission) is explained by Franck-Condon principle. ${ }^{203,204}$ It dictates that the transition to a particular vibrational level depends on the extent to which the initial and final vibrational levels resemble one another. Upon absorption, the time scale in which electronic transition occurs is many times smaller than that in which nuclei move. Hence the structure of the molecule remains unchanged during initial excitation $\left(v_{0}\right.$ of $\mathrm{GS}_{0} \rightarrow v^{\prime}{ }_{5}$ of $\left.\mathrm{S}_{1}\right)$. We call these transitions vertical excitations which are calculated to analyze absorption phenomenon. The geometry of the molecule will then relax to (level $v_{0}^{\prime}$ of $S_{1}$ according to Figure 2.2 which is) a minimum 
on the potential energy surface of the same excited state. The geometries of the excited states at these minima are optimized to study fluorescence.

\subsubsection{Technical Details of Our Methodology}

In the interest of making detailed information of the methodology readily available, some more technical details are introduced here. The SCF convergence criteria for optimization was used as it is default-implemented in Gaussian 09 and its values have been discussed in the Geometry Optimization subsection. The relativistic effects due to heavier metals such as bismuth have been treated with effective core potential (ECP) as implemented in the used basis sets def2TZVP (in case of metal cyclophane complexes) and def2SVP (in case of indium-zinc heterobimetallic complexes). In MZEDA, these effects have been treated using zeroth-order relativistic approximation (ZORA). The Hessian analysis has also been carried out for the ground state optimizations to confirm whether all the eigenvalues are positive. The presence of any single negative eigenvalue shows that the optimized structure is not a ground state structure, rather it is a transition structure (and is interconvertible to two equilibrium structures). In MZEDA, our fragmentation mode is heterolytic where one fragment consists of a single metal cation and the other is the whole cyclophane ligand. 


\section{Chapter 3}

\section{Coordination of Cyclophanes with}

\section{Heavier Main-Group Metals}

As discussed in the introduction, molecular rings such as cyclophanes have proven to be efficient hosts for metal ions in their cavities. ${ }^{116,205,206}$ They make excellent host-guest interactions commonly known as $\pi$-prismands where the cavity adopts a prism-shaped geometry to accommodate the guest metal atom. ${ }^{62,111}$ Previously, the reactivity and sensitivity of such host-guest complexes has been reported to change by replacing the aromatic ring with other different $\pi$-efficient hosts such as imidazolium. ${ }^{207}$ Moreover, cyclophanes are potential candidates as metal extractors or metal chelators from the environment perspective. ${ }^{112}$ The metal-capturing ability of cyclophanes also makes them potential candidates as raw material for ion-selective electrodes as reported in the case of [2. 2.2]paracyclophane- $\mathrm{Ag}^{+}$complex. ${ }^{112,208}$ These applications of cyclophanes revolve around the fact that they have efficient $\pi$-donation capacity and they can attract $\pi$-acceptors such as metals.

Based on these potential applications, Schmidbaur and co-workers reported several complexes of [2.2.2]paracyclophane $(p \mathrm{Cp})$ with main-group metals. ${ }^{110,116,117}$ They reported four types of complexes. One of these instances is of the type $p \mathrm{Cp} 3_{\mathrm{M}}$ where 
all the three aromatic rings of $p \mathrm{Cp}$ are occupied each from outside by a metal choride such as $\mathrm{BiCl}_{3}$ (Figure 1.12) ${ }^{110}$ or in another case $p \mathrm{Cp} 2_{\mathrm{M}}$, two phenyl rings of $p \mathrm{Cp}$ are coordinating each with a metal chloride such as $\mathrm{SbCl}_{3}$ (Figure 1.11). ${ }^{110}$ The third type $p \mathrm{Cp} 1_{\mathrm{M}}$ is characterized by the coordination of a metal chloride such as $\mathrm{AsCl}_{3}$ with the top of the cavity (Figure 1.10). ${ }^{110}$ Finally a metal cation such as $\mathrm{Ga}^{+}, \mathrm{GeCl}^{+}$ and $\mathrm{Sn}^{2+}$ has been incorporated inside the $p \mathrm{Cp}$ cavity in the type $p \mathrm{Cp}-\mathrm{M}^{\mathrm{n}+}$ as can be seen in Figures $1.7^{116}, 1.8^{117}$ and $1.9^{117}$ respectively. There is also a known example of the coordination of silver triflate with the top of the deltaphane (Dp) cavity as in Figure 1.5. ${ }^{63}$ However, the main-group metal complexes of Dp are not known to date. We based our study on these four types to computationally investigate the coordination patterns of these known and many other unknown complexes of their types with two cyclophane ligands i.e. [2.2.2]paracyclophane ( $p \mathrm{Cp})$ and deltaphane (Dp). The study includes heavier main-group metals from Groups 13 (Ga, In, Tl), 14 (Ge, Sn, Pb) and 15 (As, Sb, Bi). Hence, the current study covers a total of eight types coordination complexes (Figure 3.1) for all nine metals of interest; four with each of $p \mathrm{Cp}\left(p \mathrm{Cp} 3_{\mathrm{M}}\right.$, $\left.p C p 2_{\mathrm{M}}, p C p 1_{\mathrm{M}}, p C p-\mathrm{M}^{\mathrm{n}+}\right)$ and $\mathrm{Dp}\left(\mathrm{Dp} 3_{\mathrm{M}}, \mathrm{Dp} 2_{\mathrm{M}}, \mathrm{Dp} 1_{\mathrm{M}}, \mathrm{Dp}-\mathrm{M}^{\mathrm{n}+}\right)$. All the metals have been considered in their relatively low oxidation states i.e. 1+ for Group 13, 2+ for Group 14 and 3+ for Group 15. Although other oxidation states are known for all these metals, our study derived motivation from the scarce experimental examples where only the above-mentioned oxidation states have been reported. All the metals of our interest contain a filled $s$ orbital in the above-mentioned oxidation states.. We are also interested in the enthalpy of formation to determine the trends of thermodynamic feasibility of the complexes of interest. Moreover, Morokuma-Ziegler energy decomposition analysis (MZEDA) will be employed to discuss the bonding patterns of the inclusion complexes. The corresponding metal chlorides for all the metals used are $\mathrm{GaCl}, \mathrm{InCl}, \mathrm{TlCl}, \mathrm{GeCl}_{2}, \mathrm{SnCl}_{2}, \mathrm{PbCl}_{2}, \mathrm{AsCl}_{3}, \mathrm{SbCl}_{3}$ and $\mathrm{BiCl}_{3}$.

The calculated $\Delta \mathrm{H}_{f}$ may be further exploited in conjugation with the free energy to predict the change in entropy $(\Delta S)$ governing the formation of these complexes. The overall entropy for a chemical process is a useful indicator to check for spontaneity 
of a reaction. It is the sum of entropy of a system (Equation 3.1) and entropy of the surroundings (3.2).

$$
\begin{gathered}
\Delta S=-(\Delta G+\Delta H) / T \\
\Delta S=-\Delta H / T
\end{gathered}
$$

This means increasing exothermic enthalpy change will increase overall $\Delta \mathrm{S}$ value and vice versa. The more the positive $\Delta \mathrm{S}$, the more feasible the formation of a complex will be. On the other hand increasing endothermic enthalpy change will have a negative effect on the total entropy due to the process and this in turn makes the chemical pocess thermodynamically less feasible.

\subsection{Metal-[2.2.2]paracyclophane Complexes}

\subsubsection{Structural Features}

\subsubsection{1 [2.2.2]paracyclophane $(p \mathrm{Cp})$}

$p \mathrm{Cp}$ is one of the two cyclophanes involved in the current study, which consists of three arene rings attached in a cyclic pattern bridged through ethyl groups on their para positions. $p \mathrm{Cp}$ has a rigid structure ${ }^{119}$ and the diameter of the cavity is approximately $2.50 \AA .{ }^{113}$ The $\mathrm{D}_{3}$ symmetric ground state structure optimized at PBE0-D3/def2TZVP level of density functional theory is shown in Figure 3.2. The $\mathrm{D}_{3 h}$ structure optimized at a reasonable level of theory has also been reported previously but as a transition structure. ${ }^{111}$ 

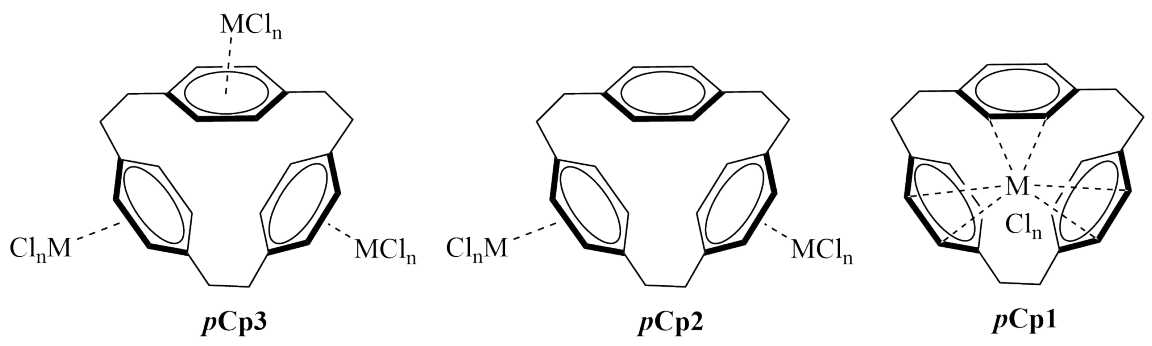

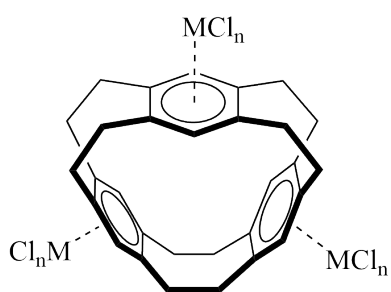

Dp3

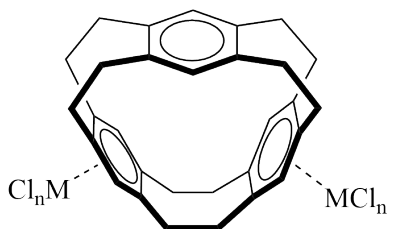

Dp2

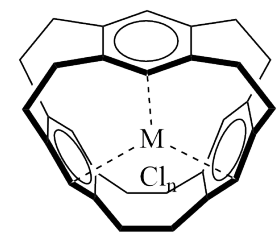

Dp1
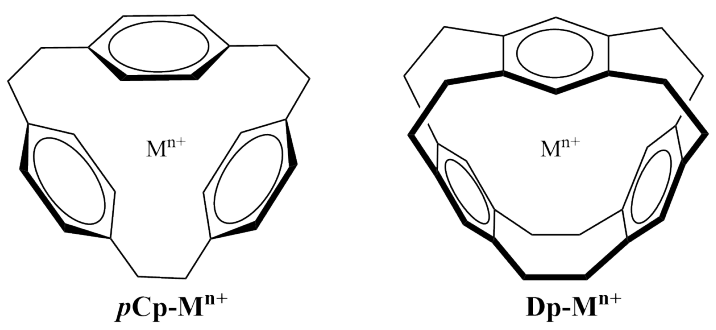

Figure 3.1: Structures of exclusion complexes: $p \mathrm{Cp}\left(\mathrm{MCl}_{\mathrm{n}}\right)_{3}\left(p \mathrm{Cp} 3_{\mathrm{M}}\right), p \mathrm{Cp}\left(\mathrm{MCl}_{\mathrm{n}}\right)_{2}$ $\left(p C p 2_{\mathrm{M}}\right), \mathrm{Dp}\left(\mathrm{MCl}_{\mathrm{n}}\right)_{3}\left(\mathrm{Dp} 3_{\mathrm{M}}\right), \mathrm{Dp}\left(\mathrm{MCl}_{\mathrm{n}}\right)_{2}\left(\mathrm{Dp} 2_{\mathrm{M}}\right)$ and inclusion complexes: $p \mathrm{CpMCl}_{\mathrm{n}}$ $\left(p C p 1_{M}\right), \mathrm{DpMCl}_{\mathrm{n}}\left(\mathrm{Dp} 1_{\mathrm{M}}\right), p \mathrm{Cp}-\mathrm{M}^{\mathrm{n}+}$ and $\mathrm{Dp}-\mathrm{M}^{\mathrm{n}+}$. In all the exclusion complexes $p \mathrm{Cp} 3_{\mathrm{M}}, p \mathrm{Cp} 2_{\mathrm{M}}, \mathrm{Dp} 3_{\mathrm{M}}$ and $\mathrm{Dp} 2_{\mathrm{M}}$ metal chlorides approach the cyclophane cavity from the outer surfaces of the aryl rings. Metal chlorides in inclusion complexes $\left(p \mathrm{Cp} 1_{\mathrm{M}}\right)$ and $\left(\mathrm{Dp} 1_{\mathrm{M}}\right)$ coordinate with the top of the cavity. Metal ions $\left(\mathrm{M}^{\mathrm{n}+}\right)$ in inclusion complexes $p C p-\mathrm{M}^{\mathrm{n}+}$ and $\mathrm{Dp}-\mathrm{M}^{\mathrm{n}+}$ are incorporated inside the cyclophane cavity.

$p \mathrm{Cp}$ bears a twisted geometry, that is evident from a 3D-image, but is still thermodynamically stable. ${ }^{62,113}$ The strain is compensated by the distortion of the ethyl fragments from their eclipsed conformation and aryl rings from their planarity. ${ }^{206}$

The available experimental data ${ }^{113}$ on the crystal structure of isolated $p \mathrm{Cp}$ is of very low resolution. However, another crystal structure $p \mathrm{Cp} . \mathrm{C}_{6} \mathrm{~N}_{4}$ (Figure 1.4 in Appendix) was reported ${ }^{114}$ in a higher resolution that can be used for comparison of both the crystals with the computational results. The experimental data in Table 3.1 shows that both the crystal structures differ from each other to a small extent. The $\mathrm{D}_{3}$ structure of $p \mathrm{Cp}$ was optimized previously ${ }^{111}$ at the same level of theory as used in the current study and no difference in the re-optimized geometry was observed. However, it is important to 
Table 3.1: Selected experimental bond lengths and dihedral angles vs their corresponding calculated values in [2.2.2]paracyclophane $(p \mathrm{Cp})$. ' $\mathrm{r}$ ' is for bond length $(\AA)$ and $\phi$ reflects a dihedral angle (degrees). It is to be noted that the optimized $\mathrm{D}_{3}$-symmetric geometry has the three bridging bond lengths identical, all the six carbon bridgeheads equivalent, equal $\mathrm{C}-\mathrm{C}$ bond lengths in the aryl rings and the alternative repetition of dihedral angles on all the six bridgeheads. Hence, all these values are written once and not repeated. The experimental data from the crystal structure of $p C p \cdot \mathrm{C}_{6} \mathrm{~N}_{4}$ is given in square brackets.

\begin{tabular}{|c|c|c|c|c|}
\hline & \multicolumn{2}{|l|}{ Experimental } & \multicolumn{2}{|c|}{ Calculated } \\
\hline & $\mathrm{r}$ & $\phi$ & $\mathrm{r}$ & $\phi$ \\
\hline (C39-C40)phenyl & $1.371[1.381]$ & & 1.391 & \\
\hline (C1-C33)bridgehead & $1.514[1.503]$ & & 1.505 & \\
\hline (C11-C14)bridging & $1.432[1.477]$ & & 1.537 & \\
\hline C33-C36-C39-C40 & & $52[66]$ & & 53 \\
\hline C6-C1-C33-C36 & & $-125[-116]$ & & -124 \\
\hline C1-C33-C36-C39 & & $39[43]$ & & 50 \\
\hline
\end{tabular}

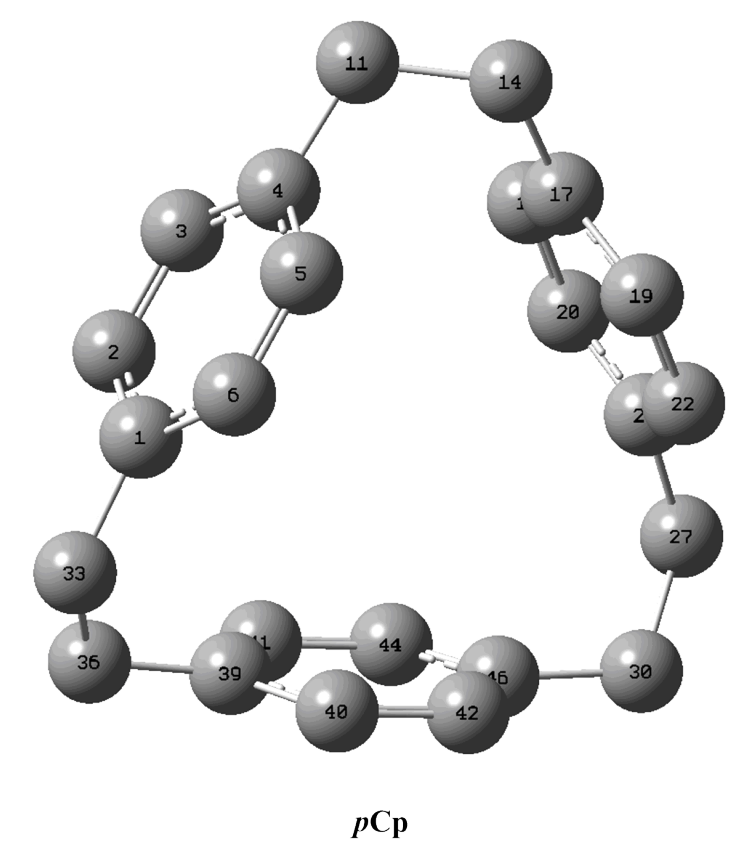

Figure 3.2: Structure of $\mathrm{D}_{3}$ symmetric $p$ Cp optimized at PBE0-D3/def2TZVP level of theory. All the atom numbering corresponds to the numbering used in discussion. Hydrogen atoms are omitted for clarity.

note down the computational data again for further comparison with the optimized complexes. Nonetheless, our calculated values of the bond distances are somewhat longer 
than the experimental ones which is consistent with the fact that gas phase (calculated) bond lengths may be longer than those in a solid-state crystal structure because of crystal packing. For instance, the calculated C-C bond length of two ethano-bridging carbon atoms is $1.537 \AA$ compared to the experimentally found $1.432 \AA$ (1.477 $\AA$ in case of $p C p . \mathrm{C}_{6} \mathrm{~N}_{4}$ crystal). Also, the calculated C-C bond length in the arene rings is all equal at $1.391 \AA$; a little longer than the average corresponding, experimentally determined bond distance of $1.371 \AA$. The $\mathrm{C}-\mathrm{C}$ bond length calculated for carbon bridgeheads is also consistent with the experimental results. Likewise, the calculated dihedral angles align with the experimental values. A comparison of calculated and experimental values for some of the bond lengths and dihedral angles is given in Table 3.1.

\subsubsection{Exclusion Complexes}

In earlier studies, the complexes of main-group metals have been reported where the coordination is with the individual phenyl cavity. ${ }^{110,209-212}$ These include two complexes involved in this study i.e. $p \mathrm{Cp} 22_{\mathrm{Sb}}$ and $p \mathrm{Cp} 3_{\mathrm{Bi}}$, therefore, their experimental bond lengths have also been given in Tables 3.9 and 3.11 respectively. We started with optimizing the exclusion complexes of all the metals where a metal chloride coordinates with the outer surface of each phenyl ring $\left(p \mathrm{Cp} 3_{\mathrm{M}}\right)$. We also optimized the ground state geometries of exclusion complexes with two metal chlorides each coordinated to an aryl ring from outer surface $\left(p \mathrm{Cp} 22_{\mathrm{M}}\right)$. Since the structural features for a group of metals $(13,14$ and 15$)$ are almost identical, the optimized geometries of only representative complexes from each group of metals are given in Figures $3.3\left(p \mathrm{Cp} 3_{\mathrm{M}}\right)$ and 3.4 $\left(p \mathrm{Cp} 22_{\mathrm{M}}\right)$.

The metal chloride in all cases prefers to remain closer to the carbon atoms of a phenyl ring that are tilted toward the center of the cavity. The metal-carbon distances in $p C \mathrm{Cp} 3_{\mathrm{M}}$ and $p \mathrm{Cp} 22_{\mathrm{M}}$ are similar for the same metal chloride. Also, there are no major differences in $\mathrm{CC}$ bond lengths in phenyl rings, bridging atoms and the carbon bridgeheads. Although the complexes of the metals belonging to the same group have similar structure 

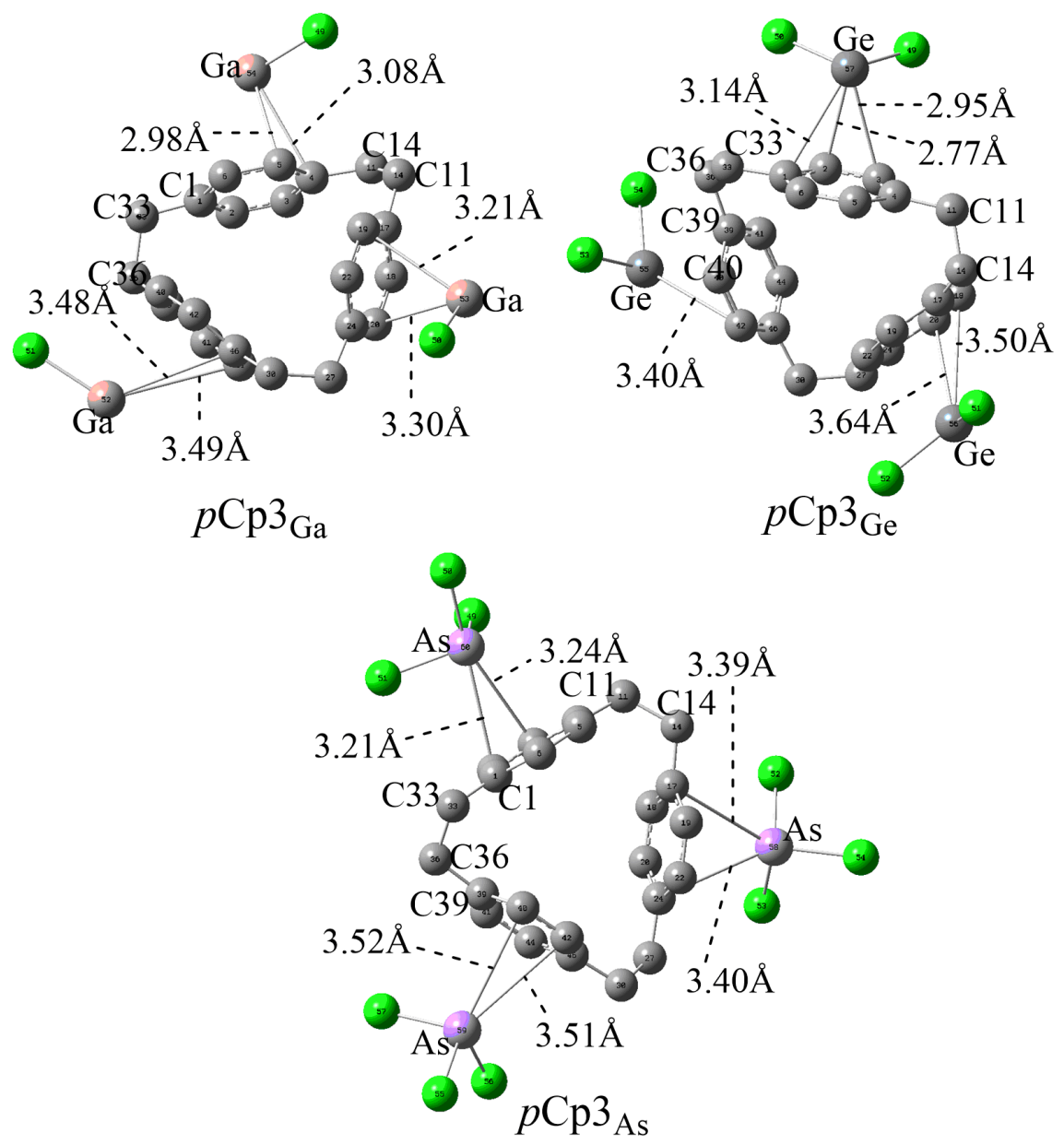

Figure 3.3: Structures of exclusion complexes of [2.2.2]paracyclophane ( $p \mathrm{Cp})$ with metal chlorides forming $p \mathrm{Cp}\left(\mathrm{MCl}_{n}\right)_{3}\left(p \mathrm{Cp} 3_{\mathrm{M}}\right)$ optimized at PBE0-D3BJ/def2TZVP level. 'M' represents Ga (for Goup 13), Ge (for Group 14) and As (for Group 15). Metal chlorides in these complexes coordinate with the outer surfaces of phenyl rings of $p \mathrm{Cp}$. It is to be noted that metal-carbon bonds have been drawn to indicate distances. For clarity, attachment of metal is shown with different carbon atoms on each phenyl ring to observe the overall position and the mode of interaction of the metal chloride. Some atom labels have been magnified as they are used in discussion in conjunction with Figure 3.2.

and mode of interaction, these behave differently from group to group. Based on the sum of the van der Waal radii, most of the exclusion complexes have M-C distances in the range such that interactions in $p \mathrm{Cp} 2_{\mathrm{M}}$ may be classified as $\eta^{6} \cdot \eta^{6}$ and in $p \mathrm{Cp} 3_{\mathrm{M}}$ as $\eta^{6} \cdot \eta^{6} . \eta^{6}$ (Tables 3.2-3.4 and 3.8-3.11), but the three-dimensional visual observations in Group $14 p \mathrm{Cp} 2_{\mathrm{M}}$ and $p \mathrm{Cp} 3_{\mathrm{M}}$ do not support this classification. This will be discussed later.

The Group 13 complexes show an $\eta^{6} \cdot \eta^{6} . \eta^{6}$ mode of interaction in case of $p \mathrm{Cp} 3_{\mathrm{M}}$ and 

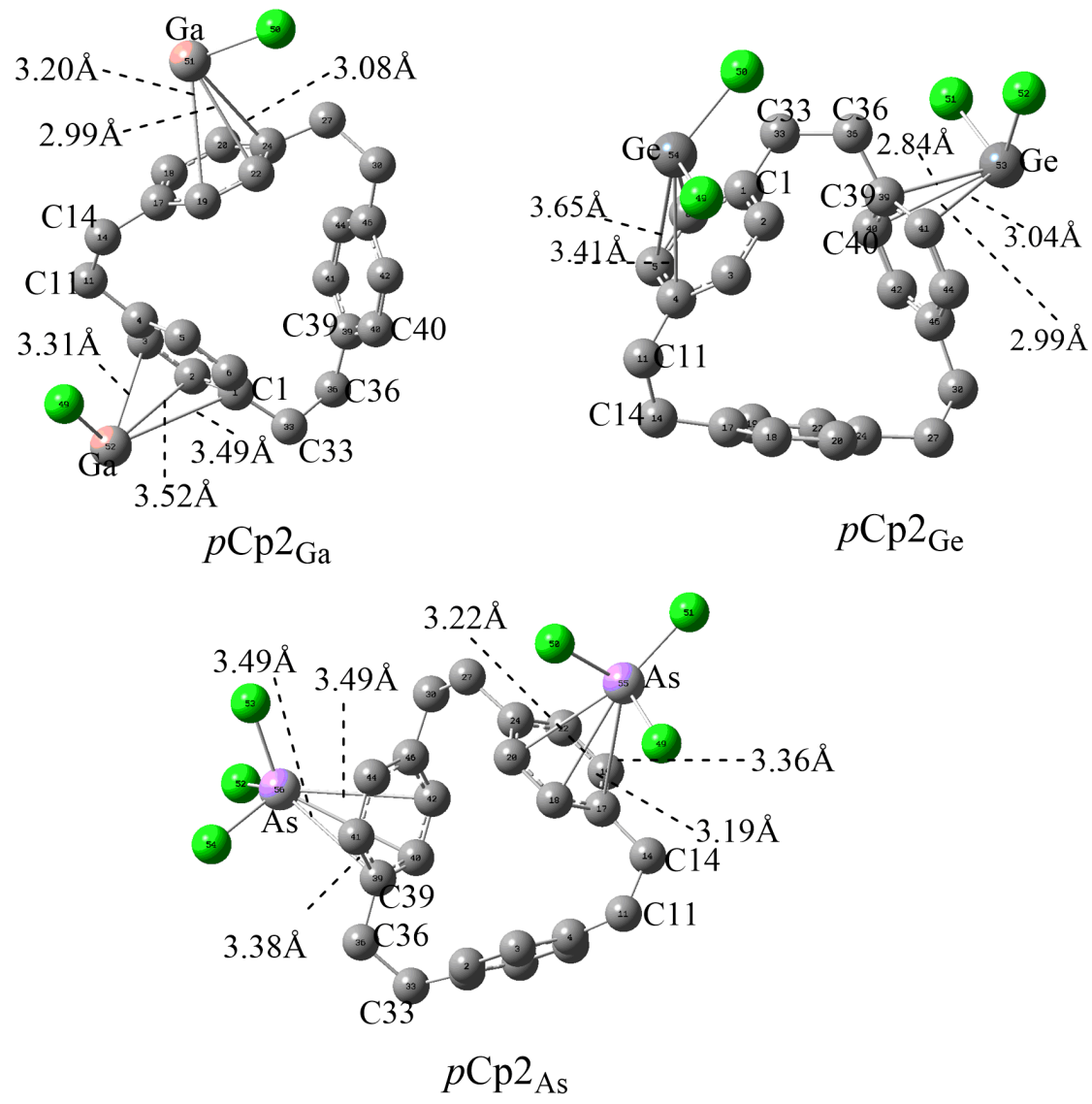

Figure 3.4: Structures of exclusion complexes of [2.2.2]paracyclophane $(p \mathrm{Cp})$ with metal chlorides forming $p \mathrm{Cp}\left(\mathrm{MCl}_{n}\right)_{2}\left(p \mathrm{Cp} 2_{\mathrm{M}}\right)$ optimized at PBE0-D3BJ/def2TZVP level. 'M' represents Ga (for Goup 13), Ge (for Group 14) and As (for Group 15). Metal chlorides in these complexes coordinate with the outer surfaces of phenyl rings of $p \mathrm{Cp}$. It is to be noted that metal-carbon bonds have been drawn to indicate distances. For clarity, attachment of metal is shown with different carbon atoms on each phenyl ring to observe the overall range of metal-carbon distances and the mode of interaction of the metal chloride.

Table 3.2: Metal-carbon distances in $p$ Cp-gallium exclusion complexes. Bold text indicates the presence of coordination based on the sum of van der Waal radii ${ }^{213}$ that is $\mathrm{Ga}$ $(1.87)+\mathrm{C}(1.70)=3.57 \AA$.

\begin{tabular}{rcc|ccc}
\hline & \multicolumn{3}{c}{$p \mathrm{Cp} 2_{\mathrm{Ga}}$} & \multicolumn{3}{c}{$p \mathrm{Cp} 3_{\mathrm{Ga}}$} \\
\hline & $\mathbf{2 . 9 5}$ & $\mathbf{2 . 9 9}$ & $\mathbf{2 . 9 8}$ & $\mathbf{3 . 0 0}$ & $\mathbf{3 . 0 0}$ \\
$\mathbf{3 . 0 5}$ & $\mathbf{3 . 0 8}$ & $\mathbf{3 . 0 8}$ & $\mathbf{3 . 0 8}$ & $\mathbf{3 . 0 9}$ \\
$\mathbf{3 . 1 8}$ & $\mathbf{3 . 2 0}$ & $\mathbf{3 . 2 0}$ & $\mathbf{3 . 2 1}$ & $\mathbf{3 . 2 1}$ \\
$\mathbf{3 . 3 1}$ & $\mathbf{3 . 2 8}$ & $\mathbf{3 . 3 2}$ & $\mathbf{3 . 3 0}$ & $\mathbf{3 . 3 0}$ \\
& $\mathbf{3 . 4 9}$ & $\mathbf{3 . 4 8}$ & $\mathbf{3 . 5 0}$ & $\mathbf{3 . 4 8}$ & $\mathbf{3 . 4 9}$ \\
& $\mathbf{3 . 5 2}$ & $\mathbf{3 . 4 9}$ & $\mathbf{3 . 5 2}$ & $\mathbf{3 . 4 9}$ & $\mathbf{3 . 5 0}$ \\
\hline Coordination & $\eta^{6} . \eta^{6}$ & \multicolumn{3}{c}{$\eta^{6} . \eta^{6} . \eta^{6}$} \\
\hline
\end{tabular}


Table 3.3: Metal-carbon distances in $p \mathrm{Cp}$-indium exclusion complexes. Bold text indicates the presence of coordination based on the sum of van der Waal radii that is In $\left(1.98^{214}\right)+\mathrm{C}\left(1.70^{215}\right)=3.68 \AA$.

\begin{tabular}{rcc|ccc}
\hline \multicolumn{2}{c}{$p \mathrm{Cp} 2_{\text {In }}$} & \multicolumn{3}{c}{$p \mathrm{Cp} 3_{\text {In }}$} \\
\hline & $\mathbf{3 . 1 3}$ & $\mathbf{3 . 1 3}$ & $\mathbf{3 . 1 5}$ & $\mathbf{3 . 1 4}$ & $\mathbf{3 . 1 3}$ \\
$\mathbf{3 . 2 3}$ & $\mathbf{3 . 2 3}$ & $\mathbf{3 . 2 4}$ & $\mathbf{3 . 2 5}$ & $\mathbf{3 . 2 4}$ \\
$\mathbf{3 . 3 2}$ & $\mathbf{3 . 3 2}$ & $\mathbf{3 . 3 5}$ & $\mathbf{3 . 3 4}$ & $\mathbf{3 . 3 3}$ \\
$\mathbf{3 . 4 6}$ & $\mathbf{3 . 4 6}$ & $\mathbf{3 . 4 5}$ & $\mathbf{3 . 4 7}$ & $\mathbf{3 . 4 8}$ \\
& $\mathbf{3 . 6 1}$ & $\mathbf{3 . 6 1}$ & $\mathbf{3 . 6 4}$ & $\mathbf{3 . 6 2}$ & $\mathbf{3 . 6 2}$ \\
& $\mathbf{3 . 6 4}$ & $\mathbf{3 . 6 4}$ & $\mathbf{3 . 6 4}$ & $\mathbf{3 . 6 5}$ & $\mathbf{3 . 6 6}$ \\
\hline Coordination & $\eta^{6} . \eta^{6}$ & \multicolumn{3}{c}{$\eta^{6} . \eta^{6} . \eta^{6}$} \\
\hline
\end{tabular}

Table 3.4: Metal-carbon distances in $p \mathrm{Cp}$-thalium exclusion complexes. Bold text indicates the presence of coordination based on the sum of van der Waal radii that is $\mathrm{Tl}$ $\left(2.08^{216}\right)+\mathrm{C}\left(1.70^{215}\right)=3.78 \AA$.

\begin{tabular}{rcc|ccc}
\hline \multicolumn{1}{c}{$p C \mathrm{Cp} 2_{\mathrm{Tl}}$} & \multicolumn{3}{c}{$p \mathrm{Cp} 3_{\mathrm{Tl}}$} \\
\hline & $\mathbf{3 . 1 6}$ & $\mathbf{3 . 1 6}$ & $\mathbf{3 . 1 7}$ & $\mathbf{3 . 1 7}$ & $\mathbf{3 . 1 6}$ \\
$\mathbf{3 . 2 5}$ & $\mathbf{3 . 2 5}$ & $\mathbf{3 . 2 7}$ & $\mathbf{3 . 2 8}$ & $\mathbf{3 . 2 7}$ \\
$\mathbf{3 . 3 5}$ & $\mathbf{3 . 3 5}$ & $\mathbf{3 . 3 7}$ & $\mathbf{3 . 3 6}$ & $\mathbf{3 . 3 6}$ \\
$\mathbf{3 . 4 8}$ & $\mathbf{3 . 4 8}$ & $\mathbf{3 . 4 8}$ & $\mathbf{3 . 5 0}$ & $\mathbf{3 . 5 1}$ \\
& $\mathbf{3 . 6 3}$ & $\mathbf{3 . 6 3}$ & $\mathbf{3 . 6 6}$ & $\mathbf{3 . 6 4}$ & $\mathbf{3 . 6 4}$ \\
& $\mathbf{3 . 6 6}$ & $\mathbf{3 . 6 6}$ & $\mathbf{3 . 6 7}$ & $\mathbf{3 . 6 7}$ & $\mathbf{3 . 6 9}$ \\
\hline Coordination & \multicolumn{3}{c}{$\eta^{6} . \eta^{6}$} & \multicolumn{3}{c}{$\eta^{6} \cdot \eta^{6}$} \\
\hline
\end{tabular}

Table 3.5: Metal-carbon distances in $p \mathrm{Cp}$-germanium exclusion complexes. Bold text indicates the presence of coordination. The sum of van der Waal radii that is $\mathrm{Ge}$ $\left(2.11^{216}\right)+\mathrm{C}\left(1.70^{215}\right)=3.81 \AA$ shows that all the M-C distances are classified as coordination but the visual representation of the complexes shows otherwise. Hence, the classification of coordination is based on three-dimensional view.

\begin{tabular}{rcc|ccc}
\hline & \multicolumn{3}{c}{$p \mathrm{Cp} 2_{\mathrm{Ge}}$} & \multicolumn{3}{c}{$p 3_{\mathrm{Ge}}$} \\
\hline $\mathbf{2 . 7 6}$ & $\mathbf{2 . 8 4}$ & $\mathbf{2 . 7 7}$ & $\mathbf{2 . 7 8}$ & $\mathbf{2 . 8 6}$ \\
$\mathbf{2 . 9 4}$ & $\mathbf{2 . 9 9}$ & $\mathbf{2 . 9 5}$ & $\mathbf{2 . 9 5}$ & $\mathbf{3 . 0 0}$ \\
$\mathbf{3 . 1 2}$ & $\mathbf{3 . 0 4}$ & $\mathbf{3 . 1 4}$ & $\mathbf{3 . 1 2}$ & $\mathbf{3 . 0 6}$ \\
3.41 & 3.37 & 3.42 & 3.41 & 3.40 \\
& 3.53 & 3.42 & 3.55 & 3.50 & 3.44 \\
& 3.65 & 3.61 & 3.67 & 3.64 & 3.64 \\
\hline Coordination & \multicolumn{3}{c}{$\eta^{3} \cdot \eta^{3} . \eta^{3} \cdot \eta^{3}$} \\
\hline
\end{tabular}

$\eta^{6} \cdot \eta^{6}$ in case of $p \mathrm{Cp} 2_{\mathrm{M}}$. The C-C bridges (in ethyl bridges) and the carbon bridgeheads retain the same bond lengths in all the Group 13 exclusion complexes as in un- 
Table 3.6: Metal-carbon distances in $p$ Cp-tin exclusion complexes. Based on the sum of van der Waal radii that is $\operatorname{Sn}\left(2.17^{213}\right)+C\left(1.70^{215}\right)=3.87 \AA$ shows that all the $\mathrm{M}-\mathrm{C}$ distances are classified as coordination but the visual representation of the complexes shows otherwise. Hence, the classification of coordination is based on threedimensional view.

\begin{tabular}{ccc|ccc}
\hline & \multicolumn{3}{c}{$p \mathrm{Cp} 2_{\mathrm{Sn}}$} & \multicolumn{3}{c}{$p 3_{\mathrm{Sn}}$} \\
\hline $\mathbf{2 . 9 8}$ & $\mathbf{2 . 9 1}$ & $\mathbf{2 . 9 4}$ & $\mathbf{3 . 0 1}$ & $\mathbf{2 . 9 3}$ \\
$\mathbf{3 . 0 8}$ & $\mathbf{3 . 0 4}$ & $\mathbf{3 . 0 9}$ & $\mathbf{3 . 0 9}$ & $\mathbf{3 . 0 8}$ \\
3.19 & 3.26 & 3.28 & 3.21 & 3.27 \\
3.44 & 3.49 & 3.54 & 3.43 & 3.52 \\
3.53 & 3.63 & 3.62 & 3.53 & 3.63 \\
& 3.68 & 3.73 & 3.74 & 3.67 & 3.74 \\
\hline Coordination & \multicolumn{3}{c}{$\eta^{2} . \eta^{2}$} & \multicolumn{3}{c}{$\eta^{2} . \eta^{2} . \eta^{2}$} \\
\hline
\end{tabular}

Table 3.7: Metal-carbon distances in $p$ Cp-lead exclusion complexes. Based on the sum of van der Waal radii that is $\mathrm{Pb}\left(2.02^{213}\right)+\mathrm{C}\left(1.70^{215}\right)=3.72 \AA$, shows that all the $\mathrm{M}-\mathrm{C}$ distances are classified as coordination but the visual representation of the complexes shows otherwise. Hence, the classification of coordination is based on threedimensional view.

\begin{tabular}{ccc|ccc}
\hline \multicolumn{2}{c}{$p \mathrm{Cp} 2_{\mathrm{Pb}}$} & \multicolumn{4}{c}{$p \mathrm{Cp} 3_{\mathrm{Pb}}$} \\
\hline & $\mathbf{3 . 0 0}$ & $\mathbf{2 . 9 4}$ & $\mathbf{2 . 9 8}$ & $\mathbf{3 . 0 1}$ & $\mathbf{2 . 9 3}$ \\
$\mathbf{3 . 0 6}$ & $\mathbf{3 . 0 3}$ & $\mathbf{3 . 0 3}$ & $\mathbf{3 . 1 1}$ & $\mathbf{3 . 0 7}$ \\
3.23 & 3.27 & 3.30 & 3.20 & 3.27 \\
3.39 & 3.44 & 3.39 & 3.45 & 3.50 \\
& 3.55 & 3.60 & 3.61 & 3.54 & 3.63 \\
& 3.66 & 3.68 & 3.64 & 3.71 & 3.72 \\
\hline Coordination & $\eta^{2} . \eta^{2}$ & \multicolumn{3}{c}{$\eta^{2} . \eta^{2} . \eta^{2}$} \\
\hline
\end{tabular}

Table 3.8: Metal-carbon distances in $p \mathrm{Cp}$-arsenic exclusion complexes. Bold text indicates the presence of coordination based on the sum of van der Waal radii that is As $\left(1.85^{213}\right)+\mathrm{C}\left(1.70^{215}\right)=3.55 \AA$.

\begin{tabular}{ccc|ccc}
\hline \multicolumn{2}{c}{$p \mathrm{Cp} 2_{\mathrm{As}}$} & \multicolumn{3}{c}{$p \mathrm{Cp} 3_{\mathrm{As}}$} \\
\hline & $\mathbf{3 . 2 0}$ & $\mathbf{3 . 1 8}$ & $\mathbf{3 . 2 2}$ & $\mathbf{3 . 2 1}$ & $\mathbf{3 . 2 2}$ \\
$\mathbf{3 . 2 2}$ & $\mathbf{3 . 2 2}$ & $\mathbf{3 . 2 3}$ & $\mathbf{3 . 2 4}$ & $\mathbf{3 . 2 3}$ \\
$\mathbf{3 . 3 8}$ & $\mathbf{3 . 3 6}$ & $\mathbf{3 . 3 9}$ & $\mathbf{3 . 3 7}$ & $\mathbf{3 . 3 9}$ \\
$\mathbf{3 . 3 7}$ & $\mathbf{3 . 4 2}$ & $\mathbf{3 . 4 1}$ & $\mathbf{3 . 4 2}$ & $\mathbf{3 . 4 1}$ \\
& $\mathbf{3 . 4 9}$ & $\mathbf{3 . 5 0}$ & $\mathbf{3 . 5 1}$ & $\mathbf{3 . 5 1}$ & $\mathbf{3 . 5 1}$ \\
& $\mathbf{3 . 4 9}$ & $\mathbf{3 . 5 2}$ & $\mathbf{3 . 5 2}$ & $\mathbf{3 . 5 3}$ & $\mathbf{3 . 5 2}$ \\
\hline Coordination & \multicolumn{3}{c}{$\eta^{6} \cdot \eta^{6} . \eta^{6} \cdot \eta^{6}$} \\
\hline
\end{tabular}


complexed $p \mathrm{Cp}$. The dihedral angles on the other hand have changed showing that the coordination on the outer surfaces of the aryl rings is accommodated by the change in geometry on the ethyl bridges. For instance, C33-C36-C39-C40 and C1-C33-C36-C39 dihedral angles change from $53^{\circ}$ and $50^{\circ}$ respectively (un-complexed $p \mathrm{Cp}$ ) to $60^{\circ}$ each in $p \mathrm{Cp} 3_{\mathrm{Ga}}$. The biggest change occurs in C6-C1-C33-C36 that undergoes a $15^{\circ}$ change from $-125^{\circ}$ to $-110^{\circ}$. The change in the dihedral angle is even more significant in $p \mathrm{Cp} 2_{\mathrm{Ga}}$ where it changes to $-104^{\circ}$.

The Group 14 metal chlorides present a case that is different to both the Groups 13 and 15 exclusion complexes. Although the sum of van der Waal radii suggest that all the $\mathrm{M}-\mathrm{C}$ distances can be classified as having coordination, a three-dimensional view of these complexes is contrary to it. Hence, the coordination in these complexes has been proposed based on the location of the corresponding metal chloride coordinating to each phenyl ring. Therefore, coordination in $p \mathrm{Cp} 2_{\mathrm{Ge}}$ is $\eta^{3} \cdot \eta^{3}$ and $\eta^{3} \cdot \eta^{3} \cdot \eta^{3}$ in $p \mathrm{Cp} 3_{\mathrm{Ge}}$ whereas it is $\eta^{2} \cdot \eta^{2}$ and $\eta^{2} \cdot \eta^{2} \cdot \eta^{2}$ in $p \mathrm{Cp} 2_{\mathrm{M}}$ and $p \mathrm{Cp} 3_{\mathrm{M}}$ type of complexes for both the other metals. We propose that there is a quasi-tetrahedral complex formation between the metal chloride and the phenyl ring in such a way that the metal-phenyl coordination is assumed as one coordination bond and the two chlorides and an a lone pair on metal make the other three corners of the tetrahedron. The $\mathrm{CC}_{\text {phenyl }}, \mathrm{CC}_{\text {bridging }}$ and $\mathrm{CC}_{\text {bridgehead }}$ bond lengths in Group 14 complexes are almost unchanged when compared to the un-complexed $p \mathrm{Cp}$. However, these complexes exhibit highly distorted dihedral angles. These changes are even more significant compared to that in their Group 13 counterparts. Each phenyl ring undergoes a different degree of distortion by rotating across the ethyl bridges. For instance, the dihedral angle of the plane C6-C1-C33$\mathrm{C} 36$ which is $-125^{\circ}$ in un-complexed $p \mathrm{Cp}$, changes to $-150^{\circ}$. The $\mathrm{C} 20-\mathrm{C} 24-\mathrm{C} 27-\mathrm{C} 30$ plane is also approximately $-125^{\circ}$ in $\mathrm{D}_{3}$ symmetric $p \mathrm{Cp}$ while in $p \mathrm{Cp} 3_{\mathrm{Ge}}$ it is $-149^{\circ}$. The C3-C4-C11-C14 and C11-C14-C17-C19 planes change from $-125^{\circ}$ to $-105^{\circ}$ and $-116^{\circ}$ respectively. The dihedral angle remains the same for the plane C33-C36-C39$\mathrm{C} 41$ in Group $14 p \mathrm{Cp} 33_{\mathrm{M}}$ while it undergoes a significant change in $p \mathrm{Cp} 2{ }_{\mathrm{M}}$ of the same group. For example it changes to $-128^{\circ}$ in $p \mathrm{Cp} 22_{\mathrm{Ge}}$. 
Table 3.9: Metal-carbon distances in $p \mathrm{Cp}$-antimony exclusion complexes. Bold text indicates the presence of coordination based on the sum of van der Waal radii that is $\mathrm{Sb}\left(2.06^{213}\right)+\mathrm{C}\left(1.70^{215}\right)=3.76 \AA . p \mathrm{Cp} 2_{\mathrm{Sb}} \cdot \mathrm{C}_{6} \mathrm{H}_{6}$ is known experimentally ${ }^{110}$ and its values are given in parentheses for comparison.

\begin{tabular}{rcc|ccc}
\hline & \multicolumn{3}{c}{$p \mathrm{Cp} 2_{\mathrm{Sb}}$} & \multicolumn{3}{c}{$p 3_{\mathrm{Sb}}$} \\
\hline & $\mathbf{3 . 2 3 1}(\mathbf{3 . 1 5 0})$ & $\mathbf{3 . 1 9 9}(\mathbf{3 . 1 7 3})$ & $\mathbf{3 . 2 4}$ & $\mathbf{3 . 2 2}$ & $\mathbf{3 . 2 5}$ \\
$\mathbf{3 . 3 0 3}(\mathbf{3 . 2 2 5})$ & $\mathbf{3 . 2 5 4}(\mathbf{3 . 2 5 0})$ & $\mathbf{3 . 2 9}$ & $\mathbf{3 . 2 8}$ & $\mathbf{3 . 3 2}$ \\
$\mathbf{3 . 3 3 9}(\mathbf{3 . 2 7 0})$ & $\mathbf{3 . 4 0 3}(\mathbf{3 . 2 8 7})$ & $\mathbf{3 . 4 4}$ & $\mathbf{3 . 4 3}$ & $\mathbf{3 . 3 6}$ \\
$\mathbf{3 . 5 2 6}(\mathbf{3 . 4 2 8})$ & $\mathbf{3 . 4 9 7}(\mathbf{3 . 3 7 0})$ & $\mathbf{3 . 5 3}$ & $\mathbf{3 . 5 1}$ & $\mathbf{3 . 5 4}$ \\
& $\mathbf{3 . 5 5 9}(\mathbf{3 . 4 5 9})$ & $\mathbf{3 . 5 9 0}(\mathbf{3 . 4 7 1})$ & $\mathbf{3 . 6 0}$ & $\mathbf{3 . 6 1}$ & $\mathbf{3 . 5 7}$ \\
& $\mathbf{3 . 6 8 2}(\mathbf{3 . 5 5 8})$ & $\mathbf{3 . 6 3 2}(\mathbf{3 . 5 0 4})$ & $\mathbf{3 . 6 4}$ & $\mathbf{3 . 6 4}$ & $\mathbf{3 . 6 9}$ \\
\hline Coordination & \multicolumn{4}{c}{$\eta^{6} . \eta^{6} . \eta^{6}$} \\
\hline
\end{tabular}

Table 3.10: Selected experimental bond lengths and dihedral angles vs their corresponding calculated values in $p \mathrm{Cp} 2_{\mathrm{Sb}}$. ' $\mathrm{r}$ ' is for bond length $(\AA)$ and $\phi$ reflects a dihedral angle (degrees).

\begin{tabular}{|c|c|c|c|c|}
\hline & \multicolumn{2}{|c|}{ Experimental } & \multicolumn{2}{|c|}{ Calculated } \\
\hline & $\mathrm{r}$ & $\phi$ & $\mathrm{r}$ & $\phi$ \\
\hline (C39-C40)phenyl & 1.393 & & 1.394 & \\
\hline (C1-C33)bridgehead & 1.514 & & 1.503 & \\
\hline (C11-C14)bridging & 1.534 & & 1.537 & \\
\hline C33-C36-C39-C40 & & 43 & & 41 \\
\hline C6-C1-C33-C36 & & -135 & & -144 \\
\hline C1-C33-C36-C39 & & 49 & & 48 \\
\hline
\end{tabular}

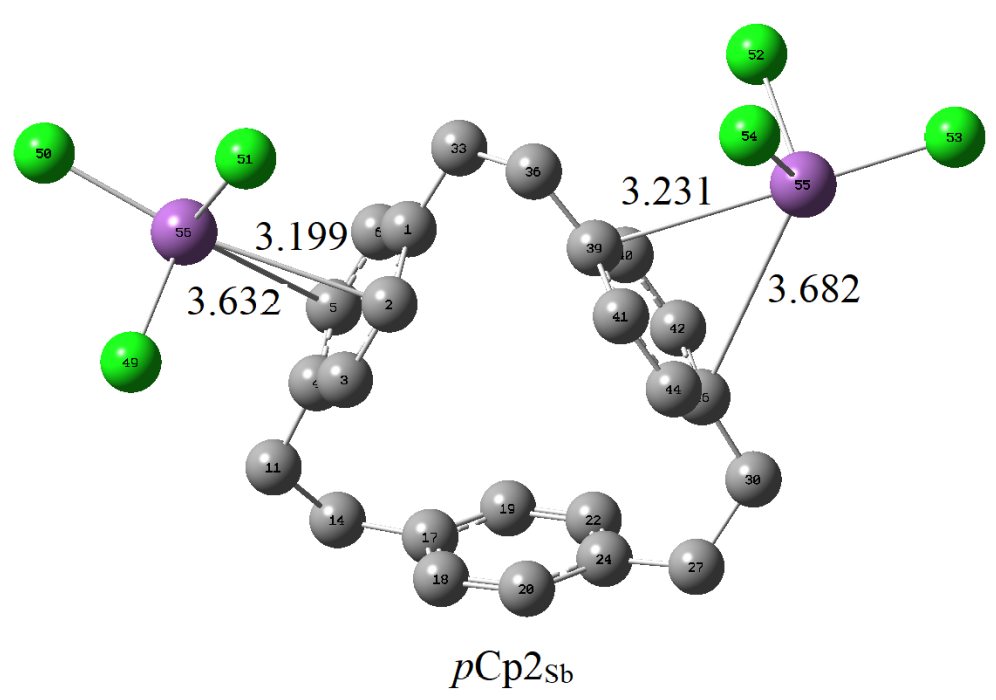

Figure 3.5: Structure of $p \mathrm{Cp} 2 \mathrm{Sb}$ optimized at PBE0-D3/def2TZVP level of theory showing the range of metal-carbon distances on each phenyl ring. 
Table 3.11: Metal-carbon distances in $p$ Cp-bismuth complexes. Bold text indicates the presence of coordination based on the sum of van der Waal radii that is $\mathrm{Bi}\left(2.07^{213}\right)+$ $\mathrm{C}\left(1.70^{215}\right)=3.77 \AA$. $p \mathrm{Cp} 3_{\mathrm{Bi}}$ is known experimentally, therefore its structural data has also been incorporated in parentheses.

\begin{tabular}{ccc|ccc}
\hline \multicolumn{4}{c}{$p C p 2_{\mathrm{Bi}}$} & \multicolumn{4}{c}{$p \mathrm{Cp} 3_{\mathrm{Bi}}$} \\
\hline & $\mathbf{3 . 2 1}$ & $\mathbf{3 . 2 4}$ & $\mathbf{3 . 3 8 2}(\mathbf{3 . 0 8 6})$ & $\mathbf{3 . 3 8 2}(\mathbf{3 . 3 3 4})$ & $\mathbf{3 . 3 8 2}(\mathbf{3 . 2 0 6})$ \\
$\mathbf{3 . 2 4}$ & $\mathbf{3 . 2 4}$ & $\mathbf{3 . 3 8 4}(\mathbf{3 . 1 3 1})$ & $\mathbf{3 . 3 8 4}(\mathbf{3 . 3 3 4})$ & $\mathbf{3 . 3 8 4}(\mathbf{3 . 2 0 9})$ \\
$\mathbf{3 . 3 6}$ & $\mathbf{3 . 3 8}$ & $\mathbf{3 . 4 2 5}(\mathbf{3 . 1 7 5})$ & $\mathbf{3 . 4 2 5}(\mathbf{3 . 3 3 8})$ & $\mathbf{3 . 4 2 5}(\mathbf{3 . 2 3 7})$ \\
& $\mathbf{3 . 4 0}$ & $\mathbf{3 . 3 8}$ & $\mathbf{3 . 5 0 0}(\mathbf{3 . 1 9 2})$ & $\mathbf{3 . 5 0 0}(\mathbf{3 . 3 7 6})$ & $\mathbf{3 . 5 0 0}(\mathbf{3 . 3 0 4})$ \\
& $\mathbf{3 . 4 8}$ & $\mathbf{3 . 4 6}$ & $\mathbf{3 . 5 4 8}(\mathbf{3 . 3 3 8})$ & $\mathbf{3 . 5 4 8}(\mathbf{3 . 4 1 7})$ & $\mathbf{3 . 5 4 8}(\mathbf{3 . 4 1 8})$ \\
& $\mathbf{3 . 5 0}$ & $\mathbf{3 . 4 7}$ & $\mathbf{3 . 6 2 4}(\mathbf{3 . 5 5 8})$ & $\mathbf{3 . 6 2 4}(\mathbf{3 . 5 1 6})$ & $\mathbf{3 . 6 2 4}(\mathbf{3 . 4 2 0})$ \\
\hline Coordination & \multicolumn{4}{c}{$\eta^{6} \cdot \eta^{6}$} & $\eta^{6} \cdot \eta^{6} . \eta^{6}$ \\
\hline
\end{tabular}

Table 3.12: Selected experimental bond lengths and dihedral angles vs their corresponding calculated values in $p \mathrm{Cp} 3_{\mathrm{Bi}}$. ' $\mathrm{r}$ ' is for bond length $(\AA)$ and $\phi$ reflects a dihedral angle (degrees).

\begin{tabular}{|c|c|c|c|c|}
\hline & \multicolumn{2}{|c|}{ Experimental } & \multicolumn{2}{|c|}{ Calculated } \\
\hline & $\mathrm{r}$ & $\phi$ & $\mathrm{r}$ & $\phi$ \\
\hline (C39-C40)phenyl & 1.383 & & 1.393 & \\
\hline (C1-C33)bridgehead & 1.519 & & 1.504 & \\
\hline (C11-C14)bridging & 1.536 & & 1.536 & \\
\hline C33-C36-C39-C40 & & -60 & & 55 \\
\hline C6-C1-C33-C36 & & 144 & & -129 \\
\hline C1-C33-C36-C39 & & -55 & & 50 \\
\hline
\end{tabular}

Group $15 p \mathrm{Cp} 2_{\mathrm{M}}$ and $p \mathrm{Cp} 3_{\mathrm{M}}$ complexes can be classified as having $\eta^{6} \cdot \eta^{6}$ and $\eta^{6} \cdot \eta^{6} \cdot \eta^{6}$ coordination respectively based on the sum of their van der Waal radii (Table 3.83.11). Group 15 metal exclusion complexes also include the experimentally known $p \mathrm{Cp} 2_{\mathrm{Sb}} \cdot \mathrm{C}_{6} \mathrm{H}_{6}$ (Figure 1.11 and $p \mathrm{Cp} 3_{\mathrm{Bi}} \cdot \mathrm{C}_{6} \mathrm{H}_{6}$ (Figure 1.12) both reported at the same time. ${ }^{110}$ Hence, the M-C distances for both these have been included in the data tables for $p \mathrm{Cp} 2_{\mathrm{Sb}}$ (Tables 3.9 and 3.10) and $p \mathrm{Cp} 3_{\mathrm{Bi}}$ (Tables 3.11 and 3.12). It is to noted that the computational analogues of both these were successfully optimized without including benzene in coordination as can be seen in the case of computational $p \mathrm{Cp} 2 \mathrm{Sb}$ (Figure 3.5) and the $\mathrm{C}_{3}$ symmetric $p \mathrm{Cp} 3_{\mathrm{Bi}}$ (Figure 3.6) complexes. The experimental bond lengths as well as the computationally calculated $\mathrm{M}-\mathrm{C}$ distances are in the range of the sum of their van der Waal radii, therefore, $p \mathrm{Cp} 2_{\mathrm{Sb}}$ has $\eta^{6} . \eta^{6}$ while $p \mathrm{Cp} 3_{\mathrm{Bi}}$ has 


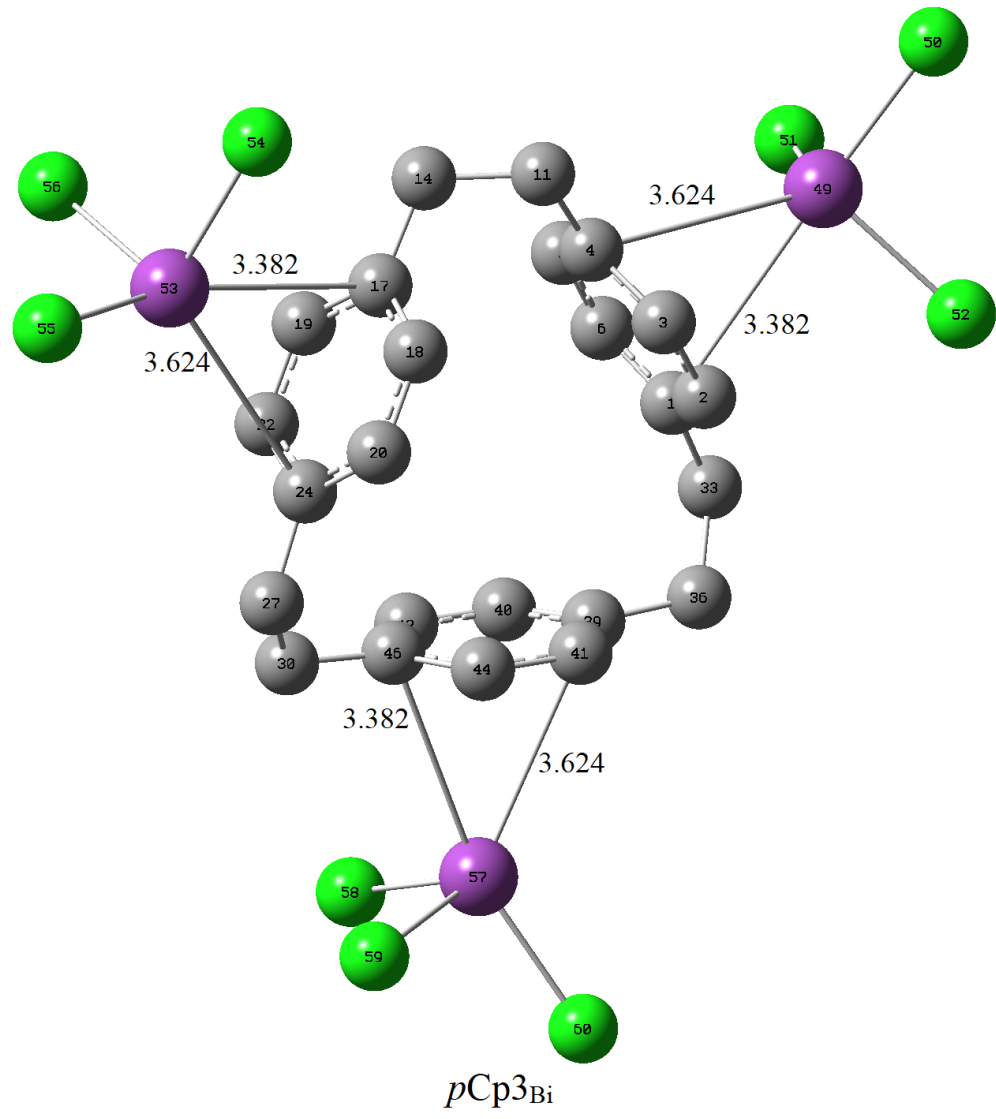

Figure 3.6: Structure of $\mathrm{C}_{3}$ symmetric $p \mathrm{Cp} 3_{\mathrm{Bi}}$ optimized at PBE0-D3/def2TZVP level of theory showing the range of metal-carbon distances on each phenyl ring.

$\eta^{6} \cdot \eta^{6} \cdot \eta^{6}$ coordination. The phenyl rings undergo distortion through the change in dihedral angles but to a smaller extent than that in complexes of the metals of Group 13 and 14. The extent of the distortion in $p C p$ exclusion complexes can be arranged in the decreasing order of $p \mathrm{Cp}_{\mathrm{M} 14}>p \mathrm{Cp}_{\mathrm{M} 13}>p \mathrm{C} \mathrm{p}_{\mathrm{M} 15}$. Figures 3.3 and 3.4 include $p \mathrm{Cp} 3_{\mathrm{As}}$ and $p \mathrm{Cp} 22_{\mathrm{As}}$ respectively as representatives from Group 15 . By carefully examining the structures, we find that the plane $\mathrm{C} 6-\mathrm{C} 1-\mathrm{C} 33-\mathrm{C} 36$ distorts from $-125^{\circ}$ (in free $p \mathrm{Cp}$ ) to $-136^{\circ}$ in $p \mathrm{Cp}_{A s}$. Moreover, the planes C33-C36-C39-C40 and $\mathrm{C} 1-\mathrm{C} 33-\mathrm{C} 36-\mathrm{C} 39$ are not affected very much in Group 15 complexes. In $p \mathrm{Cp} 2 \mathrm{As}_{\mathrm{As}} \mathrm{C} 6-\mathrm{C} 1-\mathrm{C} 33-\mathrm{C} 36$ remains approximately $-123^{\circ}$ while its counterpart on one side i.e. C20-C24-C27-C30 changes to $-110^{\circ}$ and on the other side i.e. C11-C14-C17-C19 updates to $-143^{\circ}$. 


\subsubsection{Inclusion Complexes}

The inclusion complexes $p C p 1_{\mathrm{M}}$ and $p \mathrm{Cp}-\mathrm{M}^{\mathrm{n}+}$ were optimized for all nine metals under discussion. In $p C p 1_{\mathrm{M}}$, a metal chloride coordinates with the peripheries of the $p \mathrm{Cp}$ phenyl rings from the top of the cavity. We name $p \mathrm{Cp} 1_{M}$ as inclusion complexes despite the fact that the metal chloride is still outside the cyclophane ring but the former faces the $p \mathrm{Cp}$ cavity from the top. The $p \mathrm{Cp}$ moiety accommodates the metal chlorides (in $p C \mathrm{C} 1_{\mathrm{M}}$ ) and the metal cations (in $p \mathrm{Cp}-\mathrm{M}^{\mathrm{n}+}$ ) by changing dihedral angles.

Table 3.13: Metal-carbon distances in $p \mathrm{Cp}$-gallium complexes. In $p \mathrm{Cp} 1_{\mathrm{Ga}}$, the $\mathrm{M}-\mathrm{C}$ bond distances are shown for two top of the cavity carbon atoms of each of the three arene rings. $\mathrm{Ga}^{+}$in $p \mathrm{Cp}-\mathrm{Ga}^{+}$has similar interaction with the three phenyl rings, so bond distances written just for one phenyl ring. Bold text indicates the presence of coordination based on the sum of van der Waal radii ${ }^{213}$ that is $\mathrm{Ga}(1.87)+\mathrm{C}(1.70)=$ $3.57 \AA$.

\begin{tabular}{cc|ccc|c}
\hline & \multicolumn{4}{|c}{$p \mathrm{Cp}-\mathrm{Ga}^{+}$} \\
& $p \mathrm{Cp} 1_{\mathrm{Ga}}$ & \multicolumn{3}{|c}{ Experimental } & Computational \\
\hline $\mathbf{3 . 0 3}$ & $\mathbf{2 . 9 0 8}$ & $\mathbf{2 . 9 1 5}$ & $\mathbf{2 . 9 2 7}$ & $\mathbf{2 . 9 4 8}$ \\
$\mathbf{3 . 3 6}$ & $\mathbf{2 . 9 2 0}$ & $\mathbf{2 . 9 3 9}$ & $\mathbf{2 . 9 5 9}$ & $\mathbf{2 . 9 7 6}$ \\
$\mathbf{3 . 1 3}$ & $\mathbf{2 . 9 6 9}$ & $\mathbf{2 . 9 4 7}$ & $\mathbf{2 . 9 9 4}$ & $\mathbf{2 . 9 9 2}$ \\
$\mathbf{3 . 1 6}$ & $\mathbf{2 . 9 7 1}$ & $\mathbf{3 . 0 2 3}$ & $\mathbf{3 . 0 0 0}$ & $\mathbf{3 . 0 1 3}$ \\
$\mathbf{3 . 5 3}$ & $\mathbf{3 . 0 4 8}$ & $\mathbf{3 . 0 4 7}$ & $\mathbf{3 . 0 0 4}$ & $\mathbf{3 . 0 1 4}$ \\
& 3.69 & $\mathbf{3 . 0 6 6}$ & $\mathbf{3 . 0 7 6}$ & $\mathbf{3 . 0 2 2}$ & $\mathbf{3 . 0 1 5}$ \\
\hline Coordination & $\eta^{2} . \eta^{2} \cdot \eta^{1}$ & \multicolumn{4}{|c}{$\eta^{6} \cdot \eta^{6} \cdot \eta^{6}$} \\
\hline
\end{tabular}

Table 3.14: Selected experimental bond lengths and dihedral angles vs their corresponding calculated values in $p \mathrm{Cp}-\mathrm{Ga}^{+}$. ' $\mathrm{r}$ ' is for bond length $(\AA)$ and $\phi$ reflects a dihedral angle (degrees).

\begin{tabular}{|c|c|c|c|c|}
\hline & \multicolumn{2}{|c|}{ Experimental } & \multicolumn{2}{|c|}{ Calculated } \\
\hline & $\mathrm{r}$ & $\phi$ & $\mathrm{r}$ & $\phi$ \\
\hline (C39-C40)phenyl & 1.367 & & 1.396 & \\
\hline (C1-C33)bridgehead & 1.511 & & 1.505 & \\
\hline (C11-C14)bridging & 1.535 & & 1.540 & \\
\hline C33-C36-C39-C40 & & 60 & & 63 \\
\hline C6-C1-C33-C36 & & -127 & & -137 \\
\hline C1-C33-C36-C39 & & 54 & & 53 \\
\hline
\end{tabular}

The Group $13 p C p 1_{M}$ differ from each other in coordination mode (Tables 3.13-3.16). Interestingly, $p \mathrm{Cp} 1_{\text {In }}$ is even so different from the other two that it has metal-carbon 

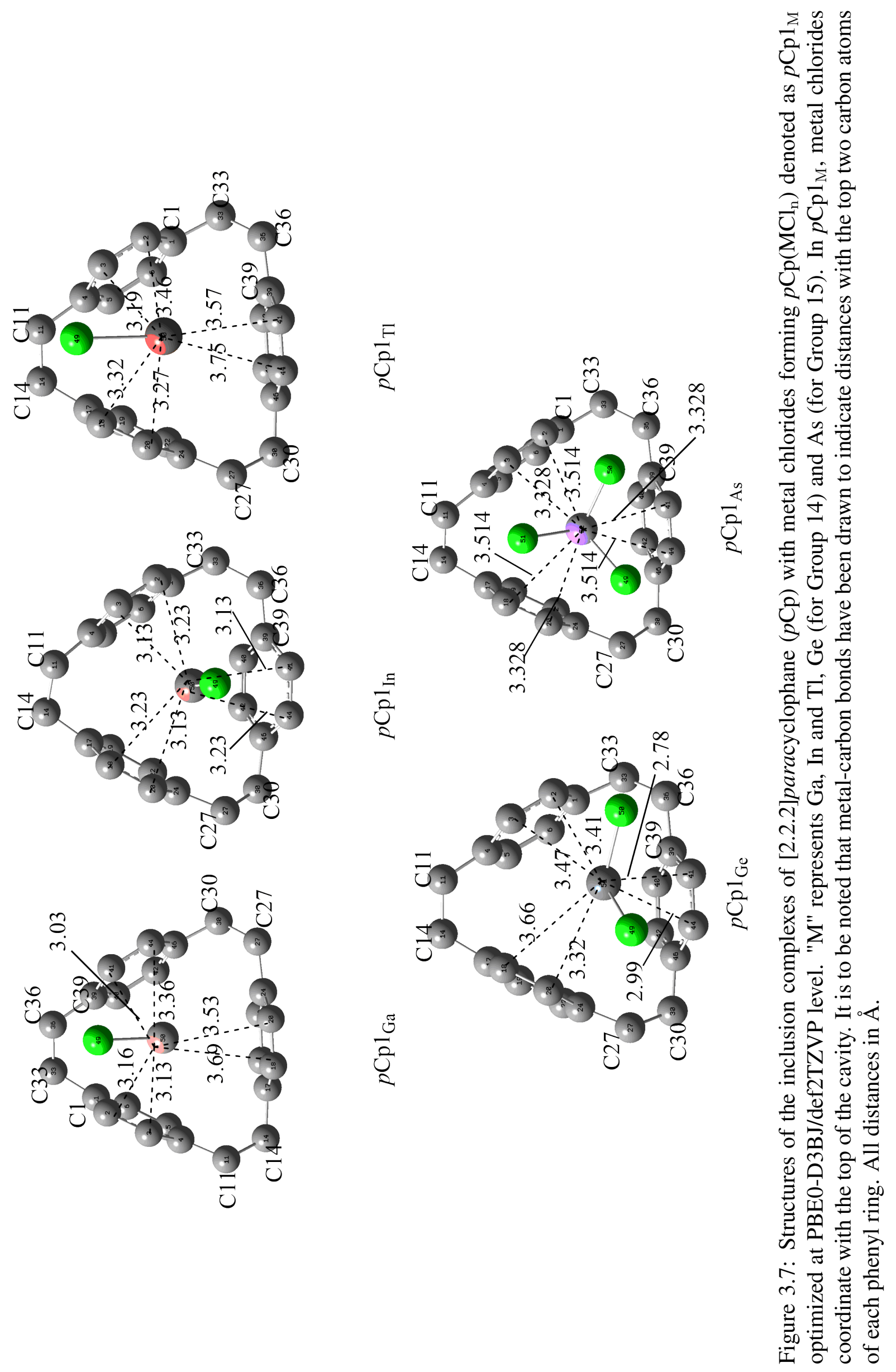


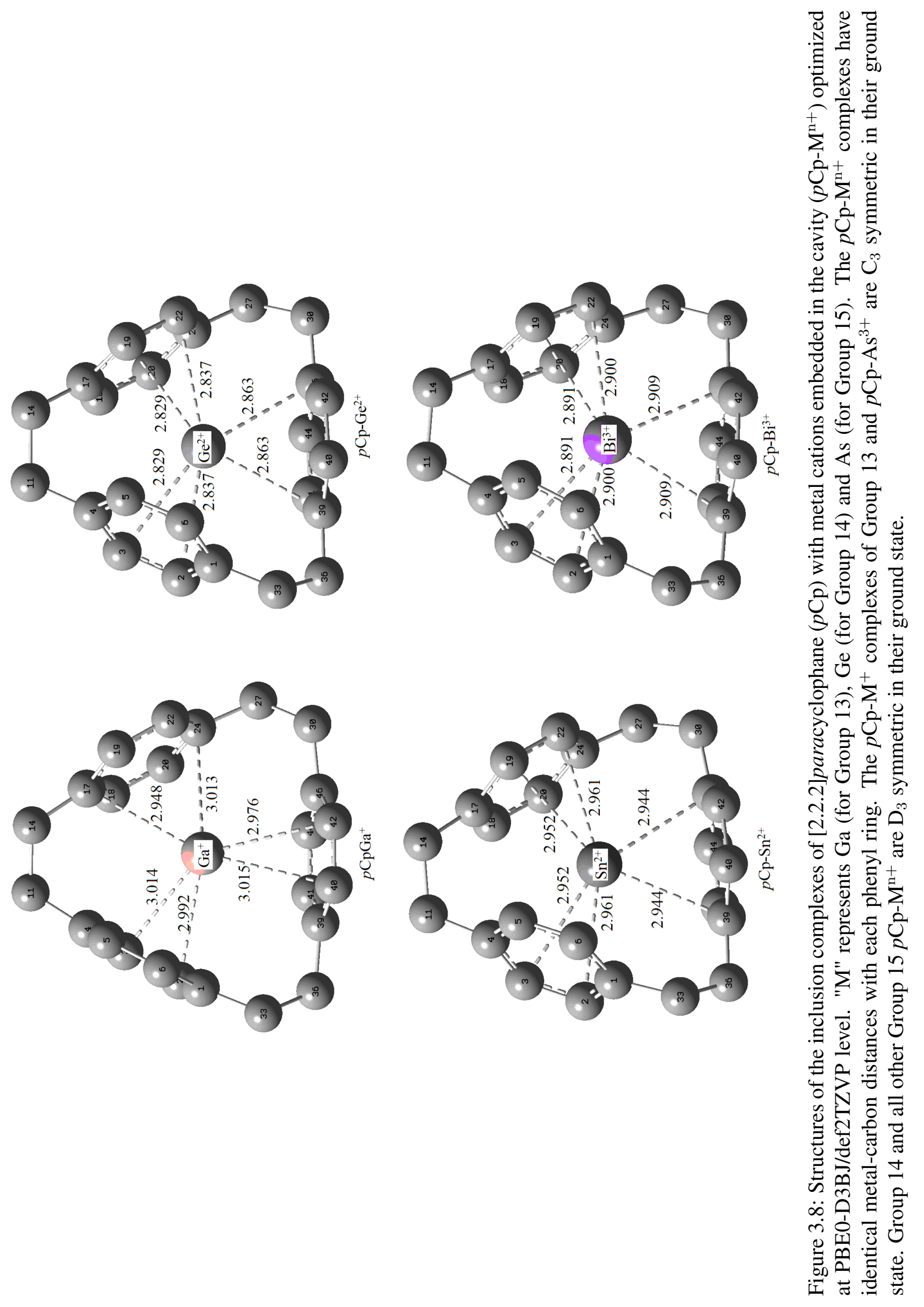


Table 3.15: Metal-carbon distances in $p \mathrm{Cp}$-indium complexes. In $p \mathrm{Cp} 1_{\mathrm{In}}$, the $\mathrm{M}-\mathrm{C}$ bond distances are shown for two top of the cavity carbon atoms of each of the three arene rings. $\mathrm{In}^{+}$has similar interaction with the three phenyl rings, so bond distances written for only one phenyl ring. Bold text indicates the presence of coordination based on the sum of van der Waal radii that is $\operatorname{In}\left(1.98^{214}\right)+C\left(1.70^{215}\right)=3.68 \AA$.

\begin{tabular}{ccc}
\hline & $p \mathrm{Cp} 1_{\text {In }}$ & $p \mathrm{Cp}-\mathrm{In}^{+}$ \\
\hline & $\mathbf{3 . 1 3}$ & $\mathbf{3 . 0 9}$ \\
& $\mathbf{3 . 2 3}$ & $\mathbf{3 . 1 4}$ \\
& $\mathbf{3 . 1 3}$ & $\mathbf{3 . 1 7}$ \\
& $\mathbf{3 . 2 3}$ & $\mathbf{3 . 3 3}$ \\
& $\mathbf{3 . 1 3}$ & $\mathbf{3 . 3 9}$ \\
& $\mathbf{3 . 2 3}$ & $\mathbf{3 . 2 8}$ \\
\hline Coordination & $\eta^{2} \cdot \eta^{2} \cdot \eta^{2}$ & $\eta^{6} \cdot \eta^{6} \cdot \eta^{6}$ \\
\hline
\end{tabular}

Table 3.16: Metal-carbon distances in $p C p$-thalium complexes inclusion complexes. In $p C p 1_{\mathrm{Tl}}$, the M-C bond distances are shown for two top of the cavity carbon atoms of each of the three arene rings. $\mathrm{Tl}^{+}$has similar interaction with the three phenyl rings, so bond distances written just for one phenyl ring. Bold text indicates the presence of coordination based on the sum of van der Waal radii that is $\mathrm{Tl}\left(2.08^{216}\right)+\mathrm{C}\left(1.70^{215}\right)=$ $3.78 \AA$.

\begin{tabular}{ccc}
\hline & $p \mathrm{Cp} 1_{\mathrm{Tl}}$ & $p \mathrm{Cp}-\mathrm{Tl}^{+}$ \\
\hline & $\mathbf{3 . 1 8}$ & $\mathbf{3 . 1 0}$ \\
& $\mathbf{3 . 2 7}$ & $\mathbf{3 . 1 9}$ \\
& $\mathbf{3 . 3 2}$ & $\mathbf{3 . 1 9}$ \\
& $\mathbf{3 . 4 6}$ & $\mathbf{3 . 3 5}$ \\
& $\mathbf{3 . 5 7}$ & $\mathbf{3 . 4 3}$ \\
& $\mathbf{3 . 7 5}$ & $\mathbf{3 . 5 0}$ \\
\hline Coordination & $\eta^{2} \cdot \eta^{2} \cdot \eta^{2}$ & $\eta^{6} \cdot \eta^{6} \cdot \eta^{6}$ \\
\hline
\end{tabular}

distances ranging from $3.13 \AA$ to $3.23 \AA$ compared to those ranging from $3.03 \AA$ to 3.69 $\AA$ in $p \mathrm{Cp} 1_{\mathrm{Ga}}$ and $3.18 \AA$ to $3.75 \AA$ in $p \mathrm{Cp} 1_{\mathrm{Tl}}$. By carefully examining the structures we conclude that $p \mathrm{Cp} 1_{\text {In }}$ involves $\eta^{2} \cdot \eta^{2} \cdot \eta^{2}$ coordination of $\mathrm{InCl}$ while $\mathrm{GaCl}$ in $p \mathrm{Cp} 1_{\mathrm{Ga}}$ interacts with five carbon atoms, thus having $\eta^{2} \cdot \eta^{2} \cdot \eta^{1}$ coordination. $\mathrm{TlCl}$ in $p \mathrm{Cp} 1_{\mathrm{Tl}}$ is located closer to one of the phenyl rings and also approaches one carbon atom of one of the other two phenyl rings, therefore it involves $\eta^{2} \cdot \eta^{2} \cdot \eta^{2}$ coordination. The $p \mathrm{Cp}$ dihedral angles are similar in $p \mathrm{Cp} 1_{\mathrm{Ga}}$ and $p \mathrm{Cp} 1_{\mathrm{Tl}}$. For example, C33-C36-C39-C40 changes from $53^{\circ}$ (in free $p \mathrm{Cp}$ ) to $35^{\circ}$ and $40^{\circ}$ respectively while $\mathrm{C} 1-\mathrm{C} 33-\mathrm{C} 36-\mathrm{C} 39$ changes from $50^{\circ}$ to $52^{\circ}$ in both. However, these planes are $30^{\circ}$ and $56^{\circ}$ respectively in 
Table 3.17: Metal-carbon distances in $p \mathrm{Cp}$-tin complexes. In $p \mathrm{Cp} 1_{\mathrm{Ge}}$, the $\mathrm{M}-\mathrm{C}$ bond distances are shown for two top of the cavity carbon atoms of each of the three arene rings. The data for the experimentally known $\mathrm{GeCl}^{+}$(Figure $1.8^{117}$ ) is also given for comparison. The Ge-C distances in this experimentally known complex vary over the top (T), bottom (B) and bridgehead (BH) phenyl carbon atoms of the cavity in a relatively broader range compared to the identical Ge-C distances in $\mathrm{D}_{3}$ symmetric optimized $p \mathrm{Cp}-\mathrm{Ge}^{2+}$ geometry. In the calculated $p \mathrm{Cp}-\mathrm{Ge}^{2+}$, the metal ion has similar interaction with the three phenyl rings, so bond distances are not repeated. Bold text indicates the presence of coordination. Based on the sum of van der Waal radii that is Ge $\left(2.11^{213}\right)+\mathrm{C}\left(1.70^{215}\right)=3.81 \AA$ shows that all the M-C distances are classified as coordination but the visual representation of the Group $14 p \mathrm{Cp} 1_{\mathrm{M}}$ complexes shows otherwise. Hence, the classification of coordination in $p \mathrm{Cp}_{\mathrm{Ge}}$ based on three-dimensional view.

\begin{tabular}{|c|c|c|c|c|c|}
\hline \multirow{2}{*}{\multicolumn{2}{|c|}{$p \mathrm{Cp} 1_{\mathrm{Ge}}$}} & \multicolumn{4}{|c|}{$p \mathrm{Cp}-\mathrm{Ge}^{2+}$} \\
\hline & & \multicolumn{3}{|c|}{ Experimental $\left(\mathrm{GeCl}^{+}\right)$} & Computational \\
\hline & 2.78 & 2.724 & 2.793 & $2.735(\mathrm{~T})$ & 2.829 \\
\hline & 2.99 & 2.733 & 2.867 & $2.802(\mathrm{~T})$ & 2.837 \\
\hline & 3.32 & 3.023 & 3.032 & $3.034(\mathrm{BH})$ & 2.863 \\
\hline & 3.41 & 3.059 & 3.171 & $3.080(\mathrm{BH})$ & 2.863 \\
\hline & 3.46 & 3.331 & 3.377 & 3.323 (B) & 2.829 \\
\hline & 3.66 & 3.334 & 3.408 & $3.297(\mathrm{~B})$ & 2.837 \\
\hline Coordination & $\eta^{2}$ & \multicolumn{3}{|c|}{$\eta^{6} \cdot \eta^{6} \cdot \eta^{6}$} & $\eta^{6} \cdot \eta^{6} \cdot \eta^{6}$ \\
\hline
\end{tabular}

Table 3.18: Selected experimental bond lengths and dihedral angles in $p \mathrm{Cp}-\mathrm{GeCl}^{+}$.$\mathrm{Al}_{4} \mathrm{Cl}_{10} \mathrm{O}_{2}{ }^{2}$ - vs their corresponding calculated values in $p \mathrm{Cp}-\mathrm{Ge}^{2+}$. ' $\mathrm{r}$ ' is for bond length $(\AA)$ and $\phi$ reflects a dihedral angle (degrees).

\begin{tabular}{|c|c|c|c|c|}
\hline & \multicolumn{2}{|c|}{ Experimental } & \multicolumn{2}{|c|}{ Calculated } \\
\hline & $\mathrm{r}$ & $\phi$ & $\mathrm{r}$ & $\phi$ \\
\hline (C39-C40)phenyl & 1.393 & & 1.398 & \\
\hline (C1-C33)bridgehead & 1.466 & & 1.497 & \\
\hline (C11-C14)bridging & 1.430 & & 1.538 & \\
\hline C33-C36-C39-C40 & & 39 & & 53 \\
\hline C6-C1-C33-C36 & & -126 & & -124 \\
\hline C1-C33-C36-C39 & & 61 & & 46 \\
\hline
\end{tabular}

$p \mathrm{Cp} 1_{\text {In }}$.

All Group 14 metal- $p$ Cp complexes have the same coordination mode in our calculated structures, so we shall discuss $p \mathrm{Cp} 1_{\mathrm{Ge}}$ here as a Group 14 representative. Pronounced differences in metal-carbon bond distances on each phenyl ring ranging from $2.78 \AA$ to $3.66 \AA$ as in Fig 3.7, indicate that $\mathrm{GeCl}_{2}$ does not coordinate equally with the three 
Table 3.19: Metal-carbon distances in $p \mathrm{Cp}$-tin complexes. In $p \mathrm{Cp}_{\mathrm{Sn}}$, the $\mathrm{M}-\mathrm{C}$ bond distances are shown for two top of the cavity carbon atoms of each of the three arene rings. The data for the experimentally known $\mathrm{GeCl}^{+}$(Figure $1.8^{117}$ ) is also given for comparison. The Ge-C distances in this experimentally known complex vary over the top (T), bottom (B) and bridgehead (BH) phenyl carbon atoms of the cavity in a relatively broader range compared to the identical Ge-C distances in $\mathrm{D}_{3}$ symmetric optimized $p \mathrm{Cp}-\mathrm{Sn}^{2+}$ geometry. In the calculated $\mathrm{Sn}^{2+}$ has similar interaction with the three phenyl rings, so bond distances have been noted down once. Bold text indicates the presence of coordination. Based on the sum of van der Waal radii that is $\operatorname{Sn}\left(2.17^{213}\right)$ $+\mathrm{C}\left(1.70^{215}\right)=3.87 \AA$ shows that all the M-C distances are classified as coordination but the visual representation of $p \mathrm{Cp} 1_{\mathrm{Sn}}$ shows otherwise. Hence, the classification of coordination is based on three-dimensional view.

\begin{tabular}{|c|c|c|c|c|c|}
\hline \multirow{2}{*}{\multicolumn{2}{|c|}{$p C p 1_{\mathrm{Sn}}$}} & \multicolumn{4}{|c|}{$p \mathrm{Cp}-\mathrm{Sn}^{2+}$} \\
\hline & & \multicolumn{3}{|c|}{ Experimental } & Computational \\
\hline & 2.78 & 2.947 & 2.816 & $2.952(\mathrm{~T})$ & 2.829 \\
\hline & 2.99 & 2.954 & 2.855 & $2.961(\mathrm{~T})$ & 2.837 \\
\hline & 3.32 & 3.007 & 2.860 & $2.944(\mathrm{BH})$ & 2.863 \\
\hline & 3.41 & 3.008 & 2.927 & $2.944(\mathrm{BH})$ & 2.863 \\
\hline & 3.46 & 3.072 & 2.937 & $2.952(\mathrm{~B})$ & 2.829 \\
\hline & 3.66 & 3.076 & 2.969 & $2.961(\mathrm{~B})$ & 2.837 \\
\hline Coordination & $\eta^{2}$ & \multicolumn{3}{|c|}{$\eta^{6} \cdot \eta^{6} \cdot \eta^{6}$} & $\eta^{6} \cdot \eta^{6} \cdot \eta^{6}$ \\
\hline
\end{tabular}

Table 3.20: Selected experimental bond lengths and dihedral angles in $p \mathrm{Cp}-\mathrm{Sn}^{2+}$.$2 \mathrm{AlCl}_{4}{ }^{-} .2 \mathrm{C}_{6} \mathrm{H}_{6}$ vs their corresponding calculated values in $p \mathrm{Cp}-\mathrm{Sn}^{2+}$. ' $\mathrm{r}$ ' is for bond length $(\AA)$ and $\phi$ reflects a dihedral angle (degrees).

\begin{tabular}{|c|c|c|c|c|}
\hline & \multicolumn{2}{|c|}{ Experimental } & \multicolumn{2}{|c|}{ Calculated } \\
\hline & $\mathrm{r}$ & $\phi$ & $\mathrm{r}$ & $\phi$ \\
\hline (C39-C40)phenyl & 1.37 & & 1.401 & \\
\hline (C1-C33)bridgehead & 1.514 & & 1.503 & \\
\hline (C11-C14)bridging & 1.432 & & 1.543 & \\
\hline C33-C36-C39-C40 & & 56 & & 52 \\
\hline C6-C1-C33-C36 & & -134 & & -126 \\
\hline C1-C33-C36-C39 & & 51 & & 51 \\
\hline
\end{tabular}

phenyl rings. Rather it develops a $\eta^{2}$ coordination with only one phenyl ring. As in all other cases, the cyclophane cavity experiences changes in dihedral angles here too. For instance, C6-C1-C33-C36 and C33-C36-C39-C40 of the un-complexed $p \mathrm{Cp}$, i.e. $-124^{\circ}$ and $53^{\circ}$ respectively, change to $-116^{\circ}$ and $28^{\circ}$ respectively in $p \mathrm{Cp} 1_{\mathrm{Ge}}$.

Group 15 metal complexes can be conveniently classified as the ones with $\eta^{2} \cdot \eta^{2} \cdot \eta^{2}$ co- 
Table 3.21: Metal-carbon distances in $p C p$-lead inclusion complexes. In $p C p 22_{\mathrm{Pb}}$, the $\mathrm{M}-\mathrm{C}$ bond distances are shown for two top of the cavity carbon atoms of each of the three arene rings. $\mathrm{Pb}^{2+}$ has similar interaction with the three phenyl rings, so bond distances written just for one phenyl ring. Bold text indicates the presence of coordination. Based on the sum of van der Waal radii that is $\mathrm{Pb}\left(2.02^{213}\right)+\mathrm{C}\left(1.70^{215}\right)=3.72$ $\AA$, shows that all the M-C distances are classified as coordination but the visual representation of the complexes shows otherwise. Hence, the classification of coordination is based on three-dimensional view.

\begin{tabular}{ccc}
\hline & $p \mathrm{Cp} 1_{\mathrm{Pb}}$ & $p \mathrm{Cp}-\mathrm{Pb}^{2+}$ \\
\hline $\mathbf{2 . 9 1}$ & $\mathbf{2 . 9 5}$ \\
$\mathbf{3 . 0 7}$ & $\mathbf{2 . 9 6}$ \\
3.27 & $\mathbf{2 . 9 6}$ \\
3.33 & $\mathbf{2 . 9 5}$ \\
& 3.38 & $\mathbf{2 . 9 6}$ \\
& 3.52 & $\mathbf{2 . 9 6}$ \\
\hline Coordination & $\eta^{2}$ & $\eta^{6} . \eta^{6} . \eta^{6}$ \\
\hline
\end{tabular}

Table 3.22: Metal-carbon distances in $p \mathrm{Cp}$-arsenic inclusion complexes. In $p \mathrm{Cp} 3_{\mathrm{As}}$, the $\mathrm{M}-\mathrm{C}$ bond distances are shown for two top of the cavity carbon atoms of each of the three arene rings. $\mathrm{As}^{3+}$ has similar interaction with the three phenyl rings, so bond distances written just for one phenyl ring. Bold text indicates the presence of coordination based on the sum of van der Waal radii that is As $\left(1.85^{213}\right)+\mathrm{C}\left(1.70^{215}\right)=$ $3.55 \AA$. $p \mathrm{Cp} 1_{\mathrm{As}}$ is known experimentally, ${ }^{110}$ so its values are also given in parentheses.

\begin{tabular}{rcc}
\hline & $p \mathrm{Cp} 1_{\mathrm{As}}$ & $p \mathrm{Cp}-\mathrm{As}^{3+}$ \\
\hline $\mathbf{3 . 3 2 8}(\mathbf{3 . 3 1 1})$ & $\mathbf{2 . 4 5}$ \\
$\mathbf{3 . 5 1 4}(\mathbf{3 . 6 0 2})$ & $\mathbf{2 . 5 2}$ \\
$\mathbf{3 . 3 2 8}(\mathbf{3 . 4 1 1})$ & $\mathbf{2 . 8 7}$ \\
$\mathbf{3 . 5 1 4}(\mathbf{3 . 5 6 3})$ & $\mathbf{2 . 9 0}$ \\
$\mathbf{3 . 3 2 8}(\mathbf{3 . 3 5 2})$ & $\mathbf{3 . 1 8}$ \\
& $\mathbf{3 . 5 1 4}(\mathbf{3 . 5 0 8})$ & $\mathbf{3 . 1 8}$ \\
\hline Coordination & $\eta^{2} \cdot \eta^{2} \cdot \eta^{2}$ & $\eta^{6} \cdot \eta^{6} \cdot \eta^{6}$ \\
\hline
\end{tabular}

ordination of respective metal chloride with the top of $p \mathrm{Cp}$ phenyl rings. Coordination in this case is also characterized by the change in $p \mathrm{Cp}$ dihedral angles. For example, the C33-C36-C39-C40 and C6-C1-C33-C36 dihedral angles in $p \mathrm{Cp} 1_{\mathrm{As}}$ are $39^{\circ}$ and $114^{\circ}$ compared to $39^{\circ}$ and $124^{\circ}$ of the un-complexed $p \mathrm{Cp} . p \mathrm{Cp} 1_{\mathrm{As}}$ is experimentally known as well. ${ }^{110}$ Its experimental data has been added to the Tables 3.22 and 3.23. It can be inferred based on the range of As-C distances that is within the sum of their van der Waal radii that $p \mathrm{Cp} 1_{\mathrm{As}}$ exhibits $\eta^{2} \cdot \eta^{2} \cdot \eta^{2}$ coordination. 
Table 3.23: Selected experimental bond lengths and dihedral angles vs their corresponding calculated $\left(\mathrm{C}_{3}\right)$ values in $p \mathrm{Cp} 1_{\mathrm{As}}$. ' $\mathrm{r}$ ' is for bond length $(\AA)$ and $\phi$ reflects a dihedral angle (degrees).

\begin{tabular}{|c|c|c|c|c|}
\hline & \multicolumn{2}{|c|}{ Experimental } & \multicolumn{2}{|c|}{ Calculated } \\
\hline & $\mathrm{r}$ & $\phi$ & $\mathrm{r}$ & $\phi$ \\
\hline (C39-C40)phenyl & 1.383 & & 1.389 & \\
\hline (C1-C33)bridgehead & 1.530 & & 1.502 & \\
\hline (C11-C14)bridging & 1.522 & & 1.535 & \\
\hline C33-C36-C39-C40 & & -46 & & 39 \\
\hline C6-C1-C33-C36 & & 132 & & -114 \\
\hline C1-C33-C36-C39 & & -51 & & 52 \\
\hline
\end{tabular}

Table 3.24: Metal-carbon distances in $p \mathrm{Cp}$-antimony complexes. In $p \mathrm{Cp} 3_{\mathrm{Sb}}$, the $\mathrm{M}-\mathrm{C}$ bond distances are shown for two top of the cavity carbon atoms of each of the three arene rings. $\mathrm{Sb}^{3+}$ has similar interaction with the three phenyl rings, so bond distances written just for one phenyl ring. Bold text indicates the presence of coordination based on the sum of van der Waal radii that is $\mathrm{Sb}\left(2.06^{213}\right)+\mathrm{C}\left(1.70^{215}\right)=3.76 \AA . p \mathrm{Cp} 2_{\mathrm{Sb}}$ is known experimentally ${ }^{110}$ and its values given in parentheses for comparison which shows that the difference in $\mathrm{M}-\mathrm{C}$ distances in both experimental and computational structures is within the range of van der Waal coordination threshold and both exhibit similar coordination.

\begin{tabular}{ccc}
\hline & $p \mathrm{Cp} 1_{\mathrm{Sb}}$ & $p \mathrm{Cp}-\mathrm{Sb}^{3+}$ \\
\hline $\mathbf{3 . 2 6}$ & $\mathbf{2 . 8 5}$ \\
$\mathbf{3 . 4 3}$ & $\mathbf{2 . 8 6}$ \\
$\mathbf{3 . 2 6}$ & $\mathbf{2 . 8 8}$ \\
$\mathbf{3 . 4 3}$ & $\mathbf{2 . 8 5}$ \\
& $\mathbf{3 . 2 6}$ & $\mathbf{2 . 8 6}$ \\
& $\mathbf{3 . 4 3}$ & $\mathbf{2 . 8 8}$ \\
\hline Coordination & $\eta^{2} \cdot \eta^{2} \cdot \eta^{2}$ & $\eta^{6} \cdot \eta^{6} \cdot \eta^{6}$ \\
\hline
\end{tabular}

$p \mathrm{Cp}-\mathrm{M}^{\mathrm{n}+}$ inclusion complexes include few known examples. We have examples of $\eta^{6} \cdot \eta^{6} \cdot \eta^{6} \mathrm{Ga}(\mathrm{I}),{ }^{116} \mathrm{Ge}(\mathrm{II}){ }^{117}$ and $\mathrm{Sn}(\mathrm{II}){ }^{117}$ inclusion complexes with $p \mathrm{Cp}$. This led us to investigate the structure and bonding properties of all these as well as hypothetical complexes of other metals of interest. Since the trends in this case are the same for a group of metals, we show one representative from each group in Figure 3.8. The optimization of only $\mathrm{C}_{3}$ symmetric ground state minima of Group 13 could have been possible, while with $\mathrm{D}_{3}$ symmetry, only the transition structures (that interconvert between two identical $\mathrm{D}_{3}$ equilibrium structures) of these were obtained. All the Group 
Table 3.25: Metal-carbon distances in $p$ Cp-bismuth complexes. In $p \mathrm{Cp} 1_{\mathrm{Bi}}$, the $\mathrm{M}-\mathrm{C}$ bond distances are shown for two top of the cavity carbon atoms of each of the three arene rings. $\mathrm{Bi}^{3+}$ has similar interaction with the three phenyl rings, so bond distances written just for one phenyl ring. Bold text indicates the presence of coordination based on the sum of van der Waal radii that is $\mathrm{Bi}\left(2.07^{213}\right)+\mathrm{C}\left(1.70^{215}\right)=3.77 \AA$. $p \mathrm{Cp} 3_{\mathrm{Bi}}$ is known experimentally, therefore its structural data has also been incorporated in parentheses. All the experimental versus computational $\mathrm{M}-\mathrm{C}$ distances are within the range of sum of van der Waal radii, so both have similar coordination mode.

\begin{tabular}{ccc}
\hline & $p \mathrm{Cp} 1_{\mathrm{Bi}}$ & $p \mathrm{Cp}-\mathrm{Bi}^{3+}$ \\
\hline & $\mathbf{3 . 2 3}$ & $\mathbf{2 . 8 9}$ \\
& $\mathbf{3 . 4 1}$ & $\mathbf{2 . 9 0}$ \\
& $\mathbf{3 . 2 3}$ & $\mathbf{2 . 8 9}$ \\
& $\mathbf{3 . 4 1}$ & $\mathbf{2 . 9 0}$ \\
& $\mathbf{3 . 2 3}$ & $\mathbf{2 . 8 9}$ \\
& $\mathbf{3 . 4 1}$ & $\mathbf{2 . 9 0}$ \\
\hline Coordination & $\eta^{2} \cdot \eta^{2} \cdot \eta^{2}$ & $\eta^{6} \cdot \eta^{6} \cdot \eta^{6}$ \\
\hline
\end{tabular}

14 and 15 metal complexes except $p C p-\mathrm{As}^{3+}$ have $\mathrm{D}_{3}$ symmetric minima. The latter is a transition structure in $\mathrm{D}_{3}$ symmetry while its ground state minimum has only been attained up to $\mathrm{C}_{3}$ symmetry as a result of optimization. All the nine $p \mathrm{Cp}-\mathrm{M}^{\mathrm{n}+}$ complexes have $\eta^{6} \cdot \eta^{6} \cdot \eta^{6}$ coordination mode based on the sum of metal-carbon van der Waal radii as evident from the Tables 3.13-3.25. However, the range of $\mathrm{M}-\mathrm{C}$ distances in $p \mathrm{Cp}-\mathrm{In}^{+}$ (Table 3.15), $p \mathrm{Cp}^{-\mathrm{Tl}^{+}}$(Table 3.16 and $p \mathrm{Cp}-\mathrm{As}^{3+}$ (Table 3.22 is relatively broader than all other $p \mathrm{Cp}-\mathrm{M}^{\mathrm{n}+}$ complexes.

As in the cases discussed previously, the formation of these complexes also involves the deviation of $p \mathrm{Cp}$ geometry from its un-complexed shape to accommodate the metal cations in the cavity. For instance, the dihedral angle C33-C36-C39-C40 experiences a $10^{\circ}$ increase in $p \mathrm{Cp}-\mathrm{Ga}^{+}$where it changes to $63^{\circ}$. Similarly, C33-C36-C39-C40 undergoes a $13^{\circ}$ change to $-137^{\circ}$. The Group $13 p \mathrm{Cp}-\mathrm{M}^{+}$complexes are $\mathrm{C}_{3}$ symmetric and the metal-carbon bond distances are not exactly identical, however, are in a very narrow range of difference and therefore $\mathrm{Ga}^{+}$is easily located in the centre of the cavity. The results for experimentally known $p \mathrm{Cp}-\mathrm{Ga}^{+} . \mathrm{AlCl}_{4}{ }^{-}$given in Figure $1.7^{116}$ have been incorporated in the Tables 3.13 and 3.14. It can be seen that in both experimental and computationally calculated structure the coordination coordination is essentially 
$\eta^{6} \cdot \eta^{6} \cdot \eta^{6}$. All metal-carbon distances in computational results fluctuate from $2.95 \AA$ to $3.02 \AA$ ) while in experimental case, they range from $2.908 \AA$ to $3.076 \AA$ i.e. well within the sum of gallium-carbon van der Waal radii as evident from Table 3.13. In $p \mathrm{Cp}$ - $\mathrm{In}^{+}$ these distances range from $3.09 \AA$ to $3.39 \AA$ while in $p \mathrm{Cp}-\mathrm{Tl}^{+}$, they range from 3.10 $\AA$ to $3.50 \AA$. The metal cation tends to move toward the top of the cavity in $p \mathrm{Cp}-\mathrm{In}^{+}$and $p \mathrm{Cp}-\mathrm{Tl}^{+}$, and one may assume that both have $\eta^{3} \cdot \eta^{3} \cdot \eta^{3}$ coordination. However, based on the sum of metal-carbon van der Waal radii, they can be classified as having $\eta^{6} \cdot \eta^{6} \cdot \eta^{6}$ coordination based on Tables 3.15 and 3.16 respectively. The strength of different interactions vary to some extent in these complexes which will be explained in Section 3.1.3.

The Group 14 p Cp- $\mathrm{M}^{2+}$ complexes present excellent examples of computationally calculated $\mathrm{D}_{3}$ symmetric comlexes with $\eta^{6} \cdot \eta^{6} \cdot \eta^{6}$ coordination. In all these cases the metal cation resides exactly in the center of the cavity and all metal-carbon distances within a complex are also essentially identical with a range of $\mathrm{M}-\mathrm{C}$ bond distances of $0.01 \AA$ difference. With the increase in metallic size down the group the $p \mathrm{Cp}$ cavity enlarges and the change in dihedral angles occurs to accommodate the metal cations. C1-C33-C36$\mathrm{C} 39$ and all its equivalent counterparts in the case of $p \mathrm{Cp}-\mathrm{Ge}^{2+}$ change to $46^{\circ}\left(50^{\circ}\right.$ in un-complexed $p \mathrm{Cp}$ as in Table 3.1)whereas C33-C36-C39-C40 and C6-C1-C33-C36 with the values $53^{\circ}$ and $124^{\circ}$ in this case remain the same as in un-complexed $p \mathrm{Cp}$. The former two of these are $52^{\circ}$ and the latter is $126^{\circ}$ in both $p \mathrm{Cp}-\mathrm{Sn}^{2+}$ and $p \mathrm{Cp}$ $\mathrm{Pb}^{2+}$. The data for experimentally known $p \mathrm{Cp}-\mathrm{GeCl}^{+} . \mathrm{Al}_{4} \mathrm{Cl}_{10} \mathrm{O}_{2}{ }^{-} .2 \mathrm{C}_{6} \mathrm{H}_{6}$ (Figure 1.8 has been added in Tables 3.17 and 3.18. In case of the crystal structure, the bonded chloride drags germanium toward the top of the cyclophane cavity. Therefore, germanium is strongly bonded with the upper carbon atoms compared to the lower ones as evident from the Table 3.17 while still coordinating with all the 18 carbon atoms of the cyclophane cavity in a broad range of M-C distances. In the $p \mathrm{Cp}-\mathrm{Sn}^{2+} .2 \mathrm{AlCl}_{4}{ }^{-} .2 \mathrm{C}_{6} \mathrm{H}_{6}$ Figure 1.9, however, $\mathrm{Sn}^{2+}$ is located in the centre of the cyclophane cavity with a narrow range of M-C distances thus preferring $\eta^{6} \cdot \eta^{6} \cdot \eta^{6}$ coordination. 
pCp- $\mathrm{M}^{3+}$ complexes of Group 15 metals exhibit an identical coordination mode except $p \mathrm{Cp}-\mathrm{As}^{3+}$ whose equilibrium structure is $\mathrm{C}_{3}$ symmetric. Based on the broad range of M-C distances, it appears to exhibit $\eta^{2} \cdot \eta^{2} \cdot \eta^{2}$, however, the M-C distances are still within the sum of arsenic-carbon van der Waal radii and show $\eta^{6} \cdot \eta^{6} \cdot \eta^{6}$ coordination (Table 3.22). However, $p \mathrm{Cp}-\mathrm{Sb}^{3+}$ and $p \mathrm{Cp}-\mathrm{Bi}^{3+}$ show $\eta^{6} \cdot \eta^{6} \cdot \eta^{6}$ within M-C distances essentially identical. The shape of the $p \mathrm{Cp}$ cavity in these two complexes changes to a small extent to let the metal cations stay inside the cavity. The major change is the bridging dihedral angle C1-C33-C36-C39 (and all its equivalent angles) from $50^{\circ}$ to $48^{\circ}$ while keeping the rest of the structure almost unchanged. However, the $C_{3}$ symmetric $p \mathrm{Cp}-\mathrm{As}^{3+}$ demands more modifications where although the above mentioned dihedral angle $\left(47^{\circ}\right)$ remains identical to the others but C6-C1-C33-C36 and C33-C36C39-C40 change to $-110^{\circ}$ and $40^{\circ}$ respectively.

\subsubsection{Thermodynamic Parameters}

In most of the experimentally reported metal complexes, the stability has been possible through the presence of a counterion as can be seen for the $\eta^{6} \cdot \eta^{6} \cdot \eta^{6}$ inclusion complex of $\mathrm{Ga}^{+}$with $[2.2 .2]$ paracyclophane further in long-range contact with $\left[\mathrm{GaBr}_{4}\right]^{-} .116$ Also, the complexes of $\mathrm{Ge}(\mathrm{II})$ and $\mathrm{Sn}(\mathrm{II})$ with the same were reported where the metal cation while residing in the cavity is in long-range contact with $\left[\mathrm{AlCl}_{4}\right]^{-}$as well. ${ }^{117}$. Also, in the reported $p \mathrm{Cp} 1_{\mathrm{As}}, p \mathrm{Cp} 2_{\mathrm{Sb}}$ and $p \mathrm{Cp} 3_{\mathrm{Bi}}$ exclusion complexes ${ }^{110}$, the corresponding metal chlorides approach the phenyl rings from the outside. This shows the metal halides counterions has consistently served for the existence of these neutral complexes. Hence, we selected some metal chlorides in our case. We first calculated the change in enthalpy of the reactions during the formation of all hypothetical $p \mathrm{Cp} 3_{\mathrm{M}}$, $p \mathrm{Cp} 2_{\mathrm{M}}$ and $p \mathrm{Cp} 1_{\mathrm{M}}$ complexes (Table 3.26) as in Equation 3.3.

$$
p \mathrm{Cp}+\mathrm{N} \cdot \mathrm{MCl}_{\mathrm{n}} \rightarrow p \mathrm{Cp}\left(\mathrm{MCl}_{\mathrm{n}}\right)_{\mathrm{N}}
$$


Here, n denotes 1, 2 and 3 for Group 13, 14 and 15 respectively. $\mathrm{N}$ represents 1, 2 and 3 for $p \mathrm{Cp} 1_{\mathrm{M}}, p \mathrm{Cp} 2_{\mathrm{M}}$ and $p \mathrm{Cp} 3_{\mathrm{M}}$ respectively

The formation of $p \mathrm{Cp}-\mathrm{M}^{\mathrm{n}+}$ is assumed to result from the dissociation of metal chloride releasing the metal cation to enter the cavity (Equation 3.4).

$$
p \mathrm{Cp}+\mathrm{MCl}_{\mathrm{n}} \rightarrow p \mathrm{Cp}-\mathrm{M}^{\mathrm{n}+}+\mathrm{nCl}^{-}
$$

$\Delta \mathrm{H}^{\circ}$ for the metal-cyclophane complexes has been recorded in Table 3.26. The highest negative values of change in enthalpy are for the complexes of the type $p C p 3_{M}$ while the least exothermic formation is in case of $p C p 1_{\mathrm{M}}$. This gives the decreasing order of $\Delta \mathrm{H}^{\circ} p \mathrm{Cp} 3_{\mathrm{M}}>p \mathrm{Cp} 2_{\mathrm{M}}>p \mathrm{Cp} 1_{\mathrm{M}}$ which means that the increasing number of metal chlorides around the $p \mathrm{Cp}$ ring enhances stability by coordinating one metal chloride to each phenyl ring thus making uniform $\pi$ donation from outside the cavity possible.

Table 3.26: Enthalpies ( $\mathrm{kcal} / \mathrm{mol}$ ) of formation of metal complexes with [2.2.2]paracyclophane $(p \mathrm{Cp})$ in the reaction $p \mathrm{Cp}+\mathrm{NMCl}_{\mathrm{n}} \rightarrow p \mathrm{Cp}\left(\mathrm{MCl}_{\mathrm{n}}\right)_{\mathrm{N}}$. Each exclusion complex is represented by $p \mathrm{CpN}_{M}$ where $\mathrm{N}=1,2$ or 3 for $p \mathrm{Cp}\left(\mathrm{MCl}_{\mathrm{n}}\right), p \mathrm{Cp}\left(\mathrm{MCl}_{\mathrm{n}}\right)_{2}, p \mathrm{Cp}\left(\mathrm{MCl}_{\mathrm{n}}\right)_{3}$ respectively and $\mathrm{M}=\mathrm{Ga}, \mathrm{In}, \mathrm{Tl}, \mathrm{Ge}, \mathrm{Sn}, \mathrm{Pb}, \mathrm{As}, \mathrm{Sb}$ and $\mathrm{Bi}$. ' $\mathrm{n}$ ' represents the number of chlorides attached to the metal in each case and its value is 1,2 and 3 for the metals of Group 13, 14 and 15 respectively. The inclusion complexes $p C p-M^{n+}$ contain $\mathrm{M}^{\mathrm{n}+}$ inside the cavity of $p \mathrm{Cp}$. They are formed as $p \mathrm{Cp}+\mathrm{MCl}_{\mathrm{n}} \rightarrow p \mathrm{Cp}-\mathrm{M}^{\mathrm{n}+}+\mathrm{nCl}^{-}$where 'n' denotes 1, 2, and 3 for the metals of Group 13,14 and 15 respectively.

\begin{tabular}{lllllllll}
\hline & $p \mathrm{Cp} 1_{\mathrm{M}}$ & $\Delta \mathrm{H}_{f}$ & $p \mathrm{Cp} 2_{\mathrm{M}}$ & $\Delta \mathrm{H}_{f}$ & $p \mathrm{Cp} 3_{\mathrm{M}}$ & $\Delta \mathrm{H}_{f}$ & $p \mathrm{Cp}-\mathrm{M}^{\mathrm{n}+}$ & $\Delta \mathrm{H}_{f}$ \\
\hline \multirow{3}{*}{ Group 13 } & $p \mathrm{Cp} 1_{G a}$ & -49.7 & $p \mathrm{Cp} 2_{G a}$ & -59.8 & $p \mathrm{Cp} 3_{G a}$ & -69.4 & $p \mathrm{Cp}-\mathrm{Ga}^{+}$ & -4.5 \\
& $p \mathrm{Cp} 1_{I n}$ & -48.9 & $p \mathrm{Cp} 2_{I n}$ & -61.9 & $p \mathrm{Cp} 3_{I n}$ & -72.2 & $p \mathrm{Cp}-\mathrm{In}^{+}$ & -5.0 \\
& $p \mathrm{Cp} 1_{T l}$ & -54.2 & $p \mathrm{Cp} 2_{T l}$ & -63.1 & $p \mathrm{Cp} 3_{T l}$ & -74.2 & $p \mathrm{Cp}-\mathrm{Tl} 1^{+}$ & -9.9 \\
\hline \multirow{3}{*}{ Group 14 } & $p \mathrm{Cp} 1_{G e}$ & -53.4 & $p \mathrm{Cp} 2_{G e}$ & -65.3 & $p \mathrm{Cp} 3_{G e}$ & -76.6 & $p \mathrm{Cp}-\mathrm{Ge}^{2+}$ & 147.9 \\
& $p \mathrm{Cp} 1_{S n}$ & -57.7 & $p \mathrm{Cp} 2_{S n}$ & -69.5 & $p \mathrm{Cp} 3_{S n}$ & -82.6 & $p \mathrm{Cp}-\mathrm{Sn}^{2+}$ & 136.1 \\
& $p \mathrm{Cp} 1_{P b}$ & -61.9 & $p \mathrm{Cp} 2_{P b}$ & -72.3 & $p \mathrm{Cp} 3_{P b}$ & -87.3 & $p \mathrm{Cp}-\mathrm{Pb}^{2+}$ & 126.1 \\
\hline \multirow{3}{*}{ Group 15 } & $p \mathrm{Cp} 1_{A s}$ & -51.4 & $p \mathrm{Cp} 2_{A s}$ & -57.0 & $p \mathrm{Cp} 3_{A s}$ & -65.7 & $p \mathrm{Cp}-\mathrm{As}^{3+}$ & 415.6 \\
& $p \mathrm{Cp} 1_{S b}$ & -57.9 & $p \mathrm{Cp} 2_{S b}$ & -62.0 & $p \mathrm{Cp} 3_{S b}$ & -72.2 & $p C \mathrm{Cp}-\mathrm{Sb}^{3+}$ & 392.3 \\
& $p \mathrm{Cp} 1_{B i}$ & -63.3 & $p \mathrm{Cp} 2_{B i}$ & -67.8 & $p \mathrm{Cp} 3_{B i}$ & -80.4 & $p \mathrm{Cp}-\mathrm{Bi}^{3+}$ & 376.9 \\
\hline
\end{tabular}

Despite an exception of $p \mathrm{Cp}_{\text {In }}$ while moving from top to bottom in a group of metals, there is a trend of increasing ease of formation of respective complexes, which is evident 
from the increasingly negative $\Delta \mathrm{H}^{\circ}$.

The values of $\Delta \mathrm{H}^{\circ}$ from left to right across a period do not follow a definite trend. We can see while moving from Group 13 to 14 ( $\mathrm{Ga}$ to $\mathrm{Ge}, \mathrm{In}$ to $\mathrm{Sn}, \mathrm{Tl}$ to $\mathrm{Pb}$ ) there is an increase in the exotherm calculated as evident from the increasing negative enthalpies. The formation of $p \mathrm{Cp} 1_{\mathrm{M}}$, however, in case of Group 15 complexes is less exothermic compared to Group 14 counterparts. $p \mathrm{Cp} 2_{\mathrm{M}}$ and $p \mathrm{Cp} 3_{\mathrm{M}}$ complex formation in the case of Group 15 is even the least exothermic except for $p \mathrm{Cp} 3_{\text {In }}$ and $p \mathrm{Cp} 2_{\text {In }}$ complexes as they are almost thermodynamically equivalent to their antimony analogues (Table 3.26). These trends are better explained if we correlate them with the corresponding geometries and the mode of coordination. The highest stability in the case of Group 14 complexes can be attributed to the $\eta^{3}$ (in case of germanium) and $\eta^{2}$ (in case of tin and lead) coordination of the metal chlorides with the phenyl rings. As mentioned earlier, this allows the formation of a quasi-tetrahedral complex that a metal chloride makes on each phenyl moiety which adds to the extra stability of the whole complex.

The formation of $p \mathrm{Cp}-\mathrm{M}^{\mathrm{n}+}$ is the most feasible in the case of Group 13 and the trend of feasibility increases down the group. A comparison across the periods shows the decrease in feasiblity of these inclusion complexes. This can be attributed to the fact that the dissociation of the only chloride from the metal in Group 13 metal chlorides is a lot easier compared to the Group $14 \mathrm{MCl}_{2}$ and Group $15 \mathrm{MCl}_{3}$ counterparts that have two and three dissociation enthalpies, respectively associated with them. Very large endothermic enthalpies of Group $15 p \mathrm{Cp}-\mathrm{M}^{\mathrm{n}+}$ complexes are indicative of the fact that the dissociation of the third chloride is the most energy-demanding.

The trend of $\Delta \mathrm{H}^{\circ}$ of reaction within a group shown in Table 3.26 may not be completely in line with the observed metal-carbon distances for $p \mathrm{Cp}-\mathrm{M}^{\mathrm{n}+}$, prompting us to calculate the enthalpies of dissociation $\left(\Delta \mathrm{H}_{\text {dissoc. }}\right)$ of all the metal chlorides that are shown in Table 3.27. Upon an increase in covalent radius down the group, we observe a decrease in the $\Delta \mathrm{H}_{\text {dissoc. }}$. This means the trends of $\Delta \mathrm{H}_{f}$ of the complexes partly depend on the dissociation of the respective metal chlorides; the greater the $\Delta \mathrm{H}_{\text {dissoc. }}$ the more 
endothermic the formation of $p \mathrm{Cp}-\mathrm{M}^{\mathrm{n}+}$. This shows that the $\Delta \mathrm{H}_{f}$ values alone may be misleading to evaluate the strength of interaction in the complexes and some more robust evaluation is required. So we carried out the energy decomposition analysis to study the strength of coordination and different individual interactions involved in the formation of each complex.

Table 3.27: $\Delta \mathrm{H}_{\text {dissoc. }}$ of metal chlorides to yield the metal cation $\left(\mathrm{M}^{\mathrm{n}+}\right)$. It decreases with the increase in covalent radii down the group. All values in $\mathrm{kcal} / \mathrm{mol}$.

\begin{tabular}{lccl}
\hline Metal & Covalent Radius & Metallic Chloride & $\Delta \mathrm{H}_{\text {dissoc. }}$ \\
\hline $\mathrm{Ga}$ & $122(3)$ & $\mathrm{GaCl}$ & 105.6 \\
$\mathrm{In}$ & $142(5)$ & $\mathrm{InCl}$ & 90.8 \\
$\mathrm{Tl}$ & $145(7)$ & $\mathrm{TlCl}$ & 82.5 \\
\hline $\mathrm{Ge}$ & $120(4)$ & $\mathrm{GeCl}_{2}$ & 451.9 \\
$\mathrm{Sn}$ & $139(4)$ & $\mathrm{SnCl}_{2}$ & 395.9 \\
$\mathrm{~Pb}$ & $146(5)$ & $\mathrm{PbCl}_{2}$ & 370.0 \\
\hline $\mathrm{As}$ & $119(4)$ & $\mathrm{AsCl}_{3}$ & 1099.3 \\
$\mathrm{Sb}$ & $139(5)$ & $\mathrm{SbCl}_{3}$ & 959.0 \\
$\mathrm{Bi}$ & $148(4)$ & $\mathrm{BiCl}_{3}$ & 901.7 \\
\hline
\end{tabular}

\subsubsection{MZEDA of the $p \mathrm{Cp}-\mathrm{M}^{\mathrm{n}+}$ Complexes}

As discussed in the previous sections, the existence of the $p \mathrm{Cp}$ inclusion complexes of $\mathrm{Ga}(\mathrm{I}), \mathrm{Ge}(\mathrm{II})$ and $\mathrm{Sn}(\mathrm{II})$ also have experimental evidence. This led us to further investigate their bonding properties and the strength of different types of interactions in them through MZEDA. We extended our investigation to all the $p \mathrm{Cp}-\mathrm{M}^{\mathrm{n}+}$ inclusion complexes involved in the current study. We used the optimized geometries of these complexes and the said analysis was carried out at the PBE0/TZ2P level of theory. The strength of coordination may be expressed through the attraction energy terms i.e. $\Delta E^{\text {ele }}$ and $\Delta E^{\text {orb }}$. It is to be noted that $\Delta E^{\text {ele }}$ throughout our discussion depicts the strength of ionic interactions while $\Delta E^{\text {orb }}$ indicates the strength of covalent attractions. The ratio of $\Delta E^{\text {orb }}$ to $\Delta E^{\text {ele }}$ tells us about the relative importance of ionic and covalent bonding. Total steric repulsion in a complex is shown as $\Delta E^{\text {Pauli }}$. Although the instantaneous interaction energy $\Delta E^{\text {int }}$ is the sum of these three energy terms, it is desirable 
to explain the interatomic interactions in terms of three separate quantities; $\Delta E^{\text {Pauli }}$, $\Delta E^{\text {ele }}$ and $\Delta E^{\text {orb }} \cdot{ }^{202} \Delta E^{\text {orb }}$ in case of a $\mathrm{D}_{3}$ symmetric structure is further decomposed to the energy due to the non-reducible terms $A_{1}, A_{2}$ and $E_{1}$. This shows different types of orbitals based on symmetry and can be useful in comparing the orbital symmetries of complexes in different point groups. In our case, we have either $\mathrm{C}_{3}$ or $\mathrm{D}_{3}$ symmetric complexes, however, such a comparison based on orbital-symmetries is not possible due to the limitations of the software that decomposes $\Delta E^{\text {orb }}$ into non-reducible terms for complexes with $\mathrm{D}_{3}$ point group but not with $\mathrm{C}_{3}$ symmetry. Nonetheless, these values have been added for the completeness of tables.

EDA values are given in Table 3.28 to 3.30. In Group $13 p \mathrm{Cp}-\mathrm{M}^{\mathrm{n}+}$ complexes, the $\Delta \mathrm{H}_{f}$ becomes increasingly exothermic moving down the group which forces one to infer that the $p \mathrm{Cp}-\mathrm{Tl}^{+}$is more stable than $p \mathrm{Cp}-\mathrm{Ga}^{+}$and $p \mathrm{Cp}-\mathrm{In}^{+}$. However, the EDA terms in Table 3.28 suggest otherwise. This is consistent with the fact that the thermodynamic parameters such as enthalpy of formation or dissociation energy can not independently explain the strength of an interaction. ${ }^{202}$ The strength of covalent interaction decreases to a small extent moving from $\mathrm{Ga}^{+}$to $\mathrm{In}^{+}$and $\mathrm{Tl}^{+}$which is in line with the $\Delta E^{\text {int }}$ value in the order $\mathrm{Ga}>\mathrm{In}>\mathrm{Tl}$. Hence, the ratio of $\Delta E^{\text {orb }}$ to $\Delta E^{\text {ele }}$ that is 1.2 for $p \mathrm{Cp}$ $\mathrm{Ga}^{+}$and 1.0 for each of $p \mathrm{Cp}-\mathrm{In}^{+}$and $p \mathrm{Cp}_{-} \mathrm{Tl}^{+}$shows that $p \mathrm{Cp}-\mathrm{Ga}^{+}$has comparatively stronger metal-ligand interaction. This can also be correlated with the shortest metalcarbon distances in $p \mathrm{Cp}-\mathrm{Ga}^{+}$. However, it is noticed that all these $p \mathrm{Cp}-\mathrm{M}^{+}$have $\mathrm{C}_{3}$ symmetric minima while the metal cations in $p \mathrm{Cp}-\mathrm{In}^{+}$and $p \mathrm{Cp}-\mathrm{Tl}^{+}$tend to come on the peripheries of the cavity compared to the central location of $\mathrm{Ga}^{+}$which is due to the comparatively smaller contribution of $\Delta E^{\text {orb }}$ in $p \mathrm{Cp}-\mathrm{In}^{+}$and $p \mathrm{Cp}-\mathrm{Tl}^{+}$.

Since we were successful in optimizing the $\mathrm{D}_{3}$ symmetric Group $14 p \mathrm{Cp}-\mathrm{M}^{2+}$ complexes of Group 14 metals, we exploited these further for MZEDA as well (Table 3.29. The covalent interaction is the strongest for $p \mathrm{Cp}-\mathrm{Ge}^{2+}(-242.16 \mathrm{kcal} / \mathrm{mol})$ and it gradually decreases down the group through $p \mathrm{Cp}-\mathrm{Sn}^{2+}(-205.31 \mathrm{kcal} / \mathrm{mol})$ to $p \mathrm{Cp}-\mathrm{Pb}^{2+}(-$ $193.83 \mathrm{kcal} / \mathrm{mol})$. The reverse is true for the strength of electrostatic interaction moving 
Table 3.28: Results of the MZEDA analysis for M-Cyclophane $\left(\mathrm{M}=\mathrm{Ga}^{+}, \mathrm{In}^{+}, \mathrm{Tl}^{+}\right)$ complexes in $\mathrm{C}_{3}$ symmetry (results for the $\mathrm{D}_{3}$ transition structures in parentheses) at the PBE0/TZ2P level. The percentage shows the contribution of an energy term in the total attraction energy which is the sum of $\Delta E^{\text {ele }}$ and $\Delta E^{\text {orb }} . \Delta E^{\text {hybrid }}$ is the component of $\Delta E^{\text {orb }}$ expressed separately in the MZEDA results. All values in $\mathrm{kcal} \mathrm{mol}^{-1}$.

\begin{tabular}{lcccccc} 
& \multicolumn{2}{c}{ Ga } & \multicolumn{2}{c}{$\mathrm{In}$} & \multicolumn{2}{c}{$\mathrm{Tl}$} \\
\hline$\Delta E^{\text {Pauli }}$ & 61.73 & $(66.40)$ & 49.24 & $(89.40)$ & 44.47 & $(88.67)$ \\
$\Delta E^{\text {ele }}$ & -56.98 & $(-59.47)$ & -49.34 & $(-70.74)$ & -45.62 & $(-68.25)$ \\
& $45.8 \%$ & $(46.1 \%)$ & $48.97 \%$ & $(52 \%)$ & $50 \%$ & $(52.5 \%)$ \\
$\Delta E^{\text {orb }}$ & -67.40 & $(-69.46)$ & -51.42 & $(-65.44)$ & -45.43 & $(-61.79)$ \\
$\Delta E^{\text {hybrid }}$ & $54.2 \%$ & $(53.9 \%)$ & $51.03 \%$ & $(48 \%)$ & $50 \%$ & $(47.5 \%)$ \\
& 17.04 & $(17.89)$ & 20.99 & $(26.18)$ & 19.01 & $(27.49)$ \\
\hline$A_{1}$ & & $(-5.52)$ & & $(-5.82)$ & & $(-5.68)$ \\
$A_{2}$ & & $(-16.06)$ & & $(-14.66)$ & & $(-14.41)$ \\
$E_{1}$ & & $(-47.88)$ & & $(-44.97)$ & & $(-41.70)$ \\
\hline$\Delta E^{\text {int }}$ & -62.66 & $(-62.53)$ & -51.51 & $(-46.78)$ & -46.57 & $(-41.37)$ \\
\hline & & & & & &
\end{tabular}

from $\mathrm{Ge}$ to $\mathrm{Pb}$. The ratio of $\Delta E^{\text {orb }}$ to $\Delta E^{\text {ele }}$ is 2.7 for $p \mathrm{Cp}-\mathrm{Ge}^{2+}$ (metal-carbon distance 2.83-2.86 $\AA$ ) while for $p \mathrm{Cp}-\mathrm{Sn}^{2+}$ (metal-carbon distance 2.94-2.96 $\AA$ ) and $p \mathrm{Cp}-\mathrm{Pb}^{2+}$ (metal-carbon distance $2.95-2.96 \AA$ ) is 2.2 and 2.0 respectively which shows that the overall interaction is the strongest on top of the group and weakens moving down. The same is suggested by the $\Delta E^{\text {int }}$ values of these complexes in Table 3.29.

Table 3.29: Results of the MZEDA analysis for M-Cyclophane $\left(\mathrm{M}=\mathrm{Ge}^{2+}, \mathrm{Sn}^{2+}, \mathrm{Pb}^{2+}\right)$ complexes in $\mathrm{D}_{3}$ symmetry at the PBE0/TZ2P level. The percentage in parentheses shows the contribution of an energy term in the total attraction energy which is the sum of $\Delta E^{\text {ele }}$ and $\Delta E^{\text {orb }} . \Delta E^{\text {hybrid }}$ is the component of $\Delta E^{\text {orb }}$ expressed separately in the MZEDA results. $A_{1}, A_{2}$ and $E_{1}$ are the non-reducible terms in which $\Delta E^{\text {orb }}$ of a $\mathrm{D}_{3}$ structure can be decomposed. All values in $\mathrm{kcal} \mathrm{mol}^{-1}$.

\begin{tabular}{lccc} 
& $\mathrm{Ge}$ & $\mathrm{Sn}$ & $\mathrm{Pb}$ \\
\hline$\Delta E^{\text {Pauli }}$ & 75.88 & 88.57 & 96.88 \\
$\Delta E^{\text {ele }}$ & $-88.12(26.7 \%)$ & $-93.57(32.3 \%)$ & $-96.56(34.2 \%)$ \\
$\Delta E^{\text {orb }}$ & $-242.16(73.3 \%)$ & $-205.31(68.7 \%)$ & $-193.83(66.8 \%)$ \\
$\Delta E^{\text {hybrid }}$ & -20.85 & -9.15 & -9.63 \\
\hline$A_{1}$ & -12.34 & -12.68 & -12.72 \\
$A_{2}$ & -63.08 & -50.57 & -48.15 \\
$E_{1}$ & -166.74 & -142.07 & -132.97 \\
\hline$\Delta E^{\text {int }}$ & -254.41 & -210.30 & -193.50 \\
\hline
\end{tabular}

$\mathrm{As}^{3+}, \mathrm{Sb}^{3+}$ and $\mathrm{Bi}^{3+}$ form the most stable complexes among all the metal cations under 
discussion (Table 3.30). The strength of coordination with $p \mathrm{Cp}$ is in the order $\mathrm{As}^{3+}$ $>\mathrm{Sb}^{3+}>\mathrm{Bi}^{3+}$. However, the $\mathrm{C}_{3}$ symmetric $p \mathrm{Cp}-\mathrm{As}^{3+}$ minimum attains the strongest interaction as a result of effective $\pi$-donation from the $p \mathrm{Cp}$ cavity due to the smallest sized $\mathrm{As}^{3+}$. In the $p \mathrm{Cp}-\mathrm{Sb}^{3+}$ and $p \mathrm{Cp}-\mathrm{Bi}^{3+} \mathrm{D}_{3}$ symmetric complexes the metal cations coordinate equally with all the phenyl rings. Here again, the covalent interactions are the strongest for the smallest sized metal cation. The factor of increasing nuclear charge along a period also justifies the highest values of $\Delta E^{\text {orb }}$ for Group $15 p \mathrm{Cp}-\mathrm{M}^{n+}$ among the selected three groups. An inter-group comparison of these orbital interactions shows that their strongest contribution is for Group 15 complexes which makes the overall covalent interaction in the complexes of this group strongest. However, precaution is needed while interpreting these results for $p \mathrm{Cp}-\mathrm{As}^{3+}$ as the ground state minimum (values given in parentheses) does not show the decomposition of $\Delta E^{\text {orb }}$ term. The ratio of $\Delta E^{\text {orb }}$ to $\Delta E^{\text {ele }}$ is 4.8 for $p \mathrm{Cp}-\mathrm{As}^{3+}, 3.8$ for $p \mathrm{Cp}-\mathrm{Sb}^{3+}$ and 3.5 for $p \mathrm{Cp}-\mathrm{Bi}^{3+}$. This is consistent with the trend of decreasing $\Delta E^{\text {int }}$ down the group.

Table 3.30: Results of the MZEDA analysis for M-Cyclophane $\left(\mathrm{M}=\mathrm{As}^{3+}, \mathrm{Sb}^{3+}, \mathrm{Bi}^{3+}\right)$ complexes in $\mathrm{D}_{3}$ symmetry (Note that the $\mathrm{D}_{3}$ symmetric As complex is a transition structure. The results for the $\mathrm{C}_{3}$ symmetric minimum are given in parentheses) at the PBE0/TZ2P level. The percentage shows the contribution of an energy term in the total attraction energy which is the sum of $\Delta E^{\text {ele }}$ and $\Delta E^{\text {orb }} . \Delta E^{\text {hybrid }}$ is the component of $\Delta E^{\mathrm{orb}}$ expressed separately in the MZEDA results. $A_{1}, A_{2}$ and $E_{1}$ are the non-reducible terms in which $\Delta E^{\text {orb }}$ of a $\mathrm{D}_{3}$ structure can be decomposed. All values in $\mathrm{kcal} \mathrm{mol}^{-1}$.

\begin{tabular}{lcccc} 
& \multicolumn{2}{c}{ As } & $\mathrm{Sb}$ & $\mathrm{Bi}$ \\
\hline$\Delta E^{\text {Pauli }}$ & 78.98 & $(130.07)$ & 103.33 & 106.87 \\
$\Delta E^{\text {ele }}$ & -118.37 & $(-132.27)$ & -127.73 & -129.40 \\
& $16.7 \%$ & $(17.2 \%)$ & $20.7 \%$ & $22.4 \%$ \\
$\Delta E^{\text {orb }}$ & -589.09 & $(-634.78)$ & -488.72 & -447.91 \\
$\Delta E^{\text {hybrid }}$ & $83.3 \%$ & $(82.8 \%)$ & $79.3 \%$ & $77.6 \%$ \\
& -66.93 & $(-77.41)$ & -51.81 & -46.96 \\
\hline$A_{1}$ & -24.47 & & -25.71 & -25.99 \\
$A_{2}$ & -166.14 & & -130.11 & -118.96 \\
$E_{1}$ & -398.48 & & -332.90 & -302.97 \\
\hline$\Delta E^{\text {int }}$ & -628.51 & $(-636.98)$ & -513.17 & -470.42 \\
\hline
\end{tabular}




\subsection{Metal-Deltaphane Complexes}

\subsubsection{Structural Properties}

\subsubsection{Free Deltaphane}

The $\mathrm{D}_{3}$ symmetric geometry of deltaphane (Dp) optimized at PBE0-D3BJ/def2TZVP level is given in Figure 3.9. The calculated bond lengths are in agreement with the experimental data ${ }^{63}$ (Table 3.31). For a detailed comparison, the experimental data of Dp1Ag (Figure 1.5 reported at the same time ${ }^{63}$ is also given in the table. As expected due to the strain imposed by the cyclic geometry, the $\mathrm{CC}$ bond lengths in the phenyl ring differ to some extent. The longest is the the side facing the so called cavity made by the two ethyl bridges and it is calculated to be $1.393 \AA$. The other two facing toward the top (and hence the two toward the bottom) are $1.387 \AA$ each for which the experimental values $(1.390(2) \AA$ and 1.387(3) $\AA$ ) show that these are similar. The terms "top" and "bottom" are referred to as Figure 3.9 where the side above the plane of paper is the top and vice versa as otherwise both the sides are similar. As seen in the experimental data in Table 3.31, Dp affords coordination with silver triflate with little changes in dihedral angles compared to the un-complexed Dp. This shows the rigid behaviour of Dp toward the formation of complexes.

Table 3.31: Key structural parameters of deltaphane. Data from Dp1Ag is given in square brackets. ' $r$ ' is for bond-length $(\AA)$ and $\phi$ shows the dihedral angle in degrees $\left(^{\circ}\right)$.

\begin{tabular}{lcccc}
\hline & \multicolumn{2}{l}{ Experimental } & \multicolumn{2}{c}{ Calculated } \\
\cline { 3 - 5 } & & $\mathrm{r}$ & $\mathrm{r}$ & $\phi$ \\
\cline { 5 - 6 } (C37-C38)phenyl & $1.393(3)[1.411(1)]$ & & 1.397 & \\
(C40-C43)phenyl & $1.387(3)[1.395(1)]$ & & 1.389 & \\
(C1-C13)bridgehead & $1.518(2)[1.504(1)]$ & & 1.503 & \\
(C13-C14)bridging & $1.531(3)[1.525(1)]$ & & 1.550 & \\
C43-C51-C52-C3 & & $-17[-11]$ & & -26 \\
C43-C41-C45-C46 & & $84[76]$ & & 91 \\
C3-C4-C46-C45 & & $-58[-63]$ & & -52 \\
\hline
\end{tabular}




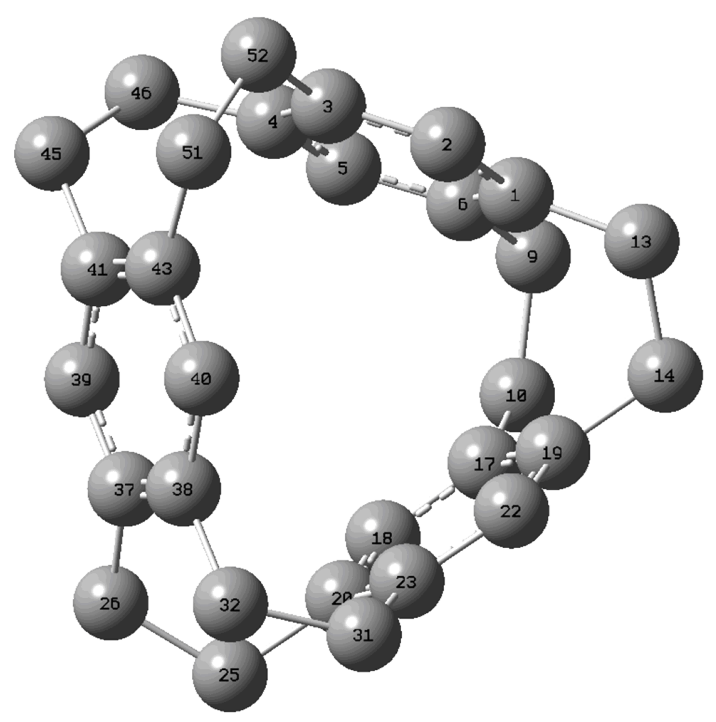

Dp

Figure 3.9: Structures of $\mathrm{D}_{3}$ symmetric Dp optimized at PBE0-D3/def2TZVP level of theory. All the atom numbering corresponds to the numbering used in discussion. Hydrogen atoms are omitted for clarity.

$\mathrm{Dp}$ is one of the two cyclophanes of our interest to investigate its ability of forming complexes with heavier main group metals through guest-host interactions. Its systematic name [2.2.2.2.2.2] $(1,2,4,5)$ cyclophane shows that it consists of three phenyl rings attached to one another through ethano bridges. Each arene ring is attached to two such bridges on either side, as distinct from the one on each para position of the phenyl rings in $p \mathrm{Cp}$. This results in high rigidity of the deltaphane geometry, but it is still able to accommodate metal cations due to its $\pi$-rich cavity engaging in donor-acceptor interactions. ${ }^{63}$

\subsubsection{Exclusion Complexes}

By analogy to the $p \mathrm{Cp}$ complexes discussed previously, we are interested in investigating the main-group metal exclusion complexes with Dp. We optimized two types of 
complexes in this case as well; $\mathrm{Dp} 3_{\mathrm{M}}$ with a metal chloride coordinating from the outer surface of each of the phenyl rings of $\mathrm{Dp}$ and the second type $\mathrm{Dp} 2_{\mathrm{M}}$ where the two metal chlorides each coordinate with one phenyl ring.

The exclusion complexes of Dp are similar in their mode of coordination to each other within a group of metals (Tables 3.32 to 3.40). Figures 3.10 and 3.11 show optimized geometries of $\mathrm{Dp} 3_{\mathrm{M}}$ and $\mathrm{Dp} 2_{\mathrm{M}}$ types of complexes of Ga (representing Group 13), $\mathrm{Ge}$ (for Group 14) and $\mathrm{As}$ (for Group 15). Although the $\mathrm{CC}_{\text {phenyl }}, \mathrm{CC}_{\text {bridging }}$ and $\mathrm{CC}_{\text {bridgeheads }}$ bond lengths remain almost identical to the un-complexed $\mathrm{Dp}$, the phenyl rings experience distortion of different magnitude in the three cases and the metalcarbon distances are different too.

Table 3.32: Metal-carbon distances in Dp-gallium exclusion complexes. In $\mathrm{Dp} 1_{\mathrm{Ga}}$, the $\mathrm{M}-\mathrm{C}$ bond distances are shown for one top of the cavity carbon atom of each of the three arene rings. $\mathrm{Ga}^{+}$in $\mathrm{Dp}-\mathrm{Ga}^{+}$has similar interaction with the three phenyl rings, so bond distances written just for one phenyl ring. Bold text indicates the presence of coordination based on the sum of van der Waal radii ${ }^{213}$ that is $\mathrm{Ga}(1.87)+\mathrm{C}(1.70)=$ $3.57 \AA$.

\begin{tabular}{cccc|ccc|c}
\hline & $\mathrm{Dp} 1_{\mathrm{Ga}}$ & \multicolumn{2}{c}{$\mathrm{Dp} 2_{\mathrm{Ga}}$} & \multicolumn{3}{c}{$\mathrm{Dp} 3_{\mathrm{Ga}}$} & $\mathrm{Dp}-\mathrm{Ga}^{+}$ \\
\hline & $\mathbf{3 . 3 4}$ & $\mathbf{3 . 0 1}$ & $\mathbf{3 . 0 0}$ & $\mathbf{3 . 0 3}$ & $\mathbf{3 . 0 2}$ & $\mathbf{3 . 0 3}$ & $\mathbf{2 . 8 1}$ \\
& $\mathbf{3 . 4 4}$ & $\mathbf{3 . 0 4}$ & $\mathbf{3 . 0 4}$ & $\mathbf{3 . 0 6}$ & $\mathbf{3 . 0 6}$ & $\mathbf{3 . 0 6}$ & $\mathbf{2 . 8 5}$ \\
& $\mathbf{3 . 5 7}$ & $\mathbf{3 . 1 6}$ & $\mathbf{3 . 1 6}$ & $\mathbf{3 . 1 7}$ & $\mathbf{3 . 1 8}$ & $\mathbf{3 . 1 8}$ & $\mathbf{2 . 8 5}$ \\
& & $\mathbf{3 . 1 8}$ & $\mathbf{3 . 1 8}$ & $\mathbf{3 . 2 0}$ & $\mathbf{3 . 2 0}$ & $\mathbf{3 . 2 0}$ & $\mathbf{2 . 8 1}$ \\
& & $\mathbf{3 . 3 6}$ & $\mathbf{3 . 3 6}$ & $\mathbf{3 . 3 6}$ & $\mathbf{3 . 3 7}$ & $\mathbf{3 . 3 7}$ & $\mathbf{2 . 8 5}$ \\
Coordination & $\eta^{1} \cdot \eta^{1} \cdot \eta^{1}$ & $\mathbf{3 . 4 0}$ & $\mathbf{3 . 4 0}$ & $\mathbf{3 . 4 1}$ & $\mathbf{3 . 4 2}$ & $\mathbf{3 . 4 2}$ & $\mathbf{2 . 8 5}$ \\
\hline
\end{tabular}

The $\mathrm{Dp} 3_{\mathrm{M}}$ in case of Group 13 and 15 have metal-carbon distances within a wide range but still within the sum of van der Waal radii. So they are engaged in $\eta^{6} \cdot \eta^{6} \cdot \eta^{6}$ coordination of each metal chloride with the phenyl ring. Also, there is no significant deviation of planes of the Dp moiety from the un-complexed Dp in both types. The rigidity of Dp makes it able to resist the significant changes in dihedral angles even in Group 14 metal complexes unlike $p \mathrm{Cp}$ complexes of $\mathrm{Ge}, \mathrm{Sn}$ and $\mathrm{Pb}$ where we observed large distortions in the cyclophane geometry. For example, in the case of Dp3 $3_{\mathrm{Ge}}, \mathrm{C} 43-\mathrm{C} 51-\mathrm{C} 52-\mathrm{C} 3$ and $\mathrm{C} 43-\mathrm{C} 41-\mathrm{C} 45-\mathrm{C} 46$ dihedral angles are $-27^{\circ}$ and $92^{\circ}$ respectively compared to $-26^{\circ}$ and 

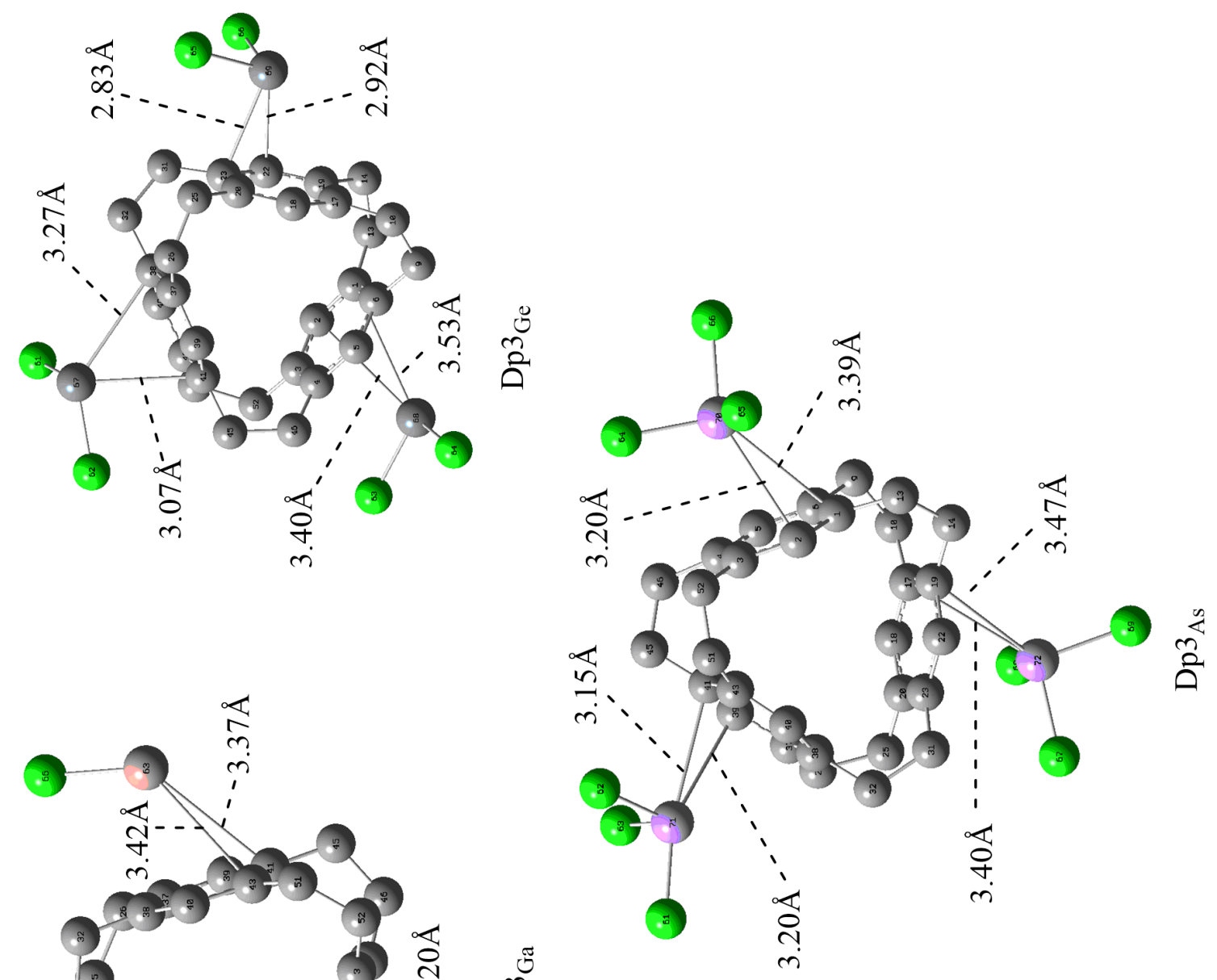

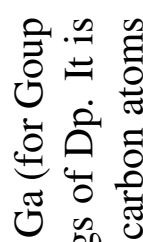

苞泀苛

运苞

은 들

$\sum_{i}$

它氖

กิ

$e_{\bar{s}}$

के

$\sum \cong$

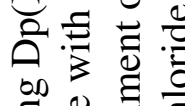

$\stackrel{00}{\Xi}$ 苞 专

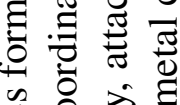

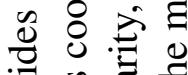

훙 ᅯ

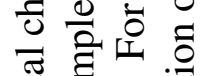

迎 है

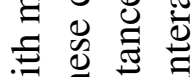

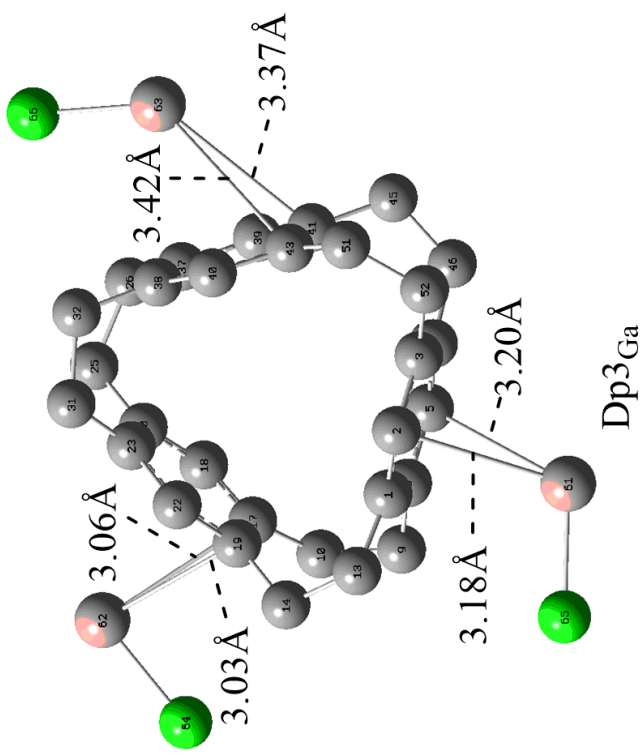

하의

คิ

记 :

는 은

坖

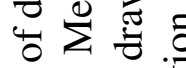
论 융

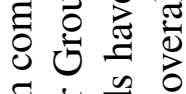
. 包艺递 屯 ปै

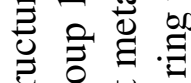
吉过芯 $\ddot{0} \stackrel{0}{0}$ ن̊ 竞笛。 

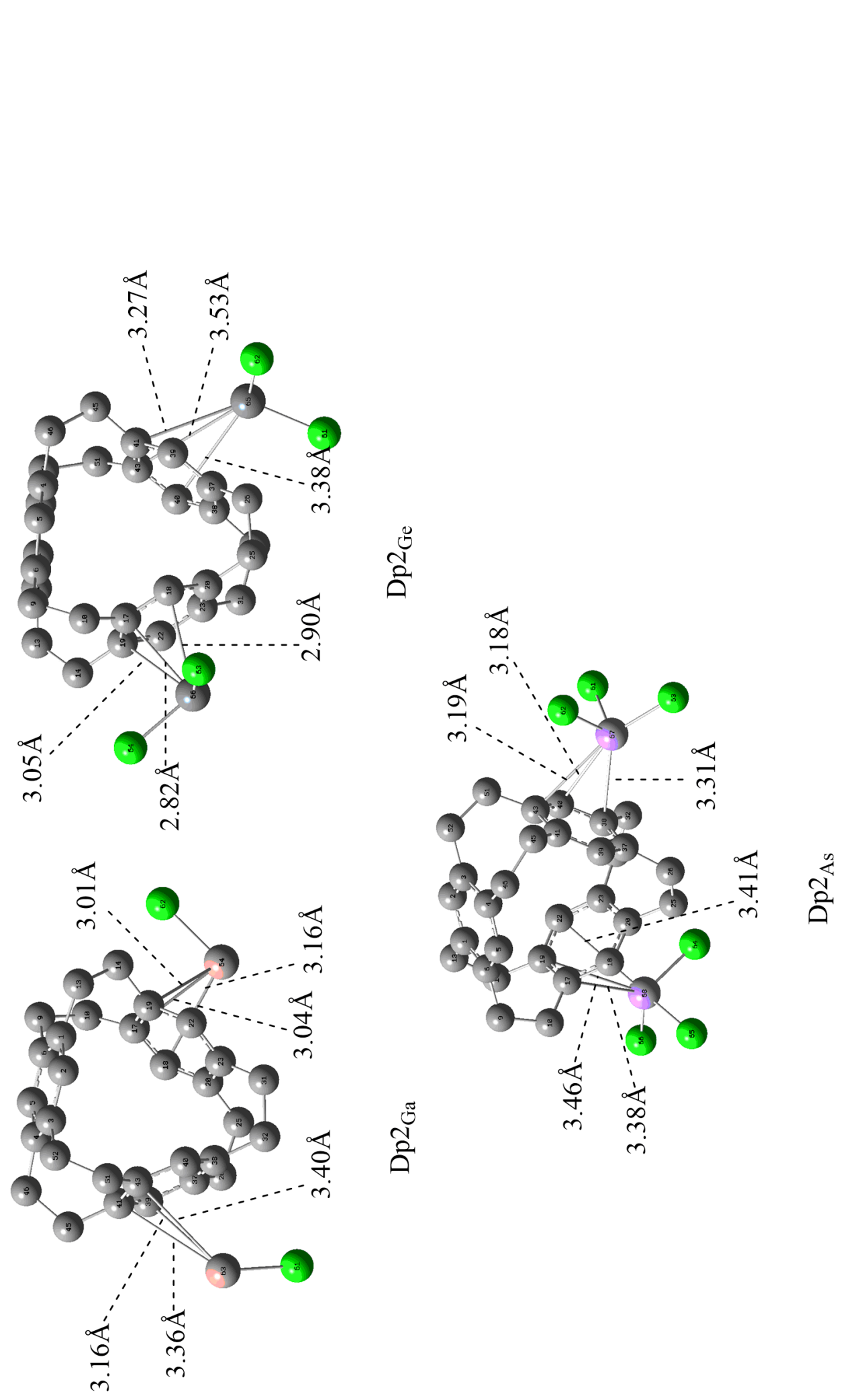

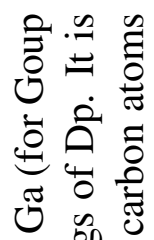

营毛苛

詷

년

岕

들 क

e

零

胥

会青泀

ఏ

总司焉

s :

응 吾

등 훙ㅎㅁ

플 홍

ヨ 00.

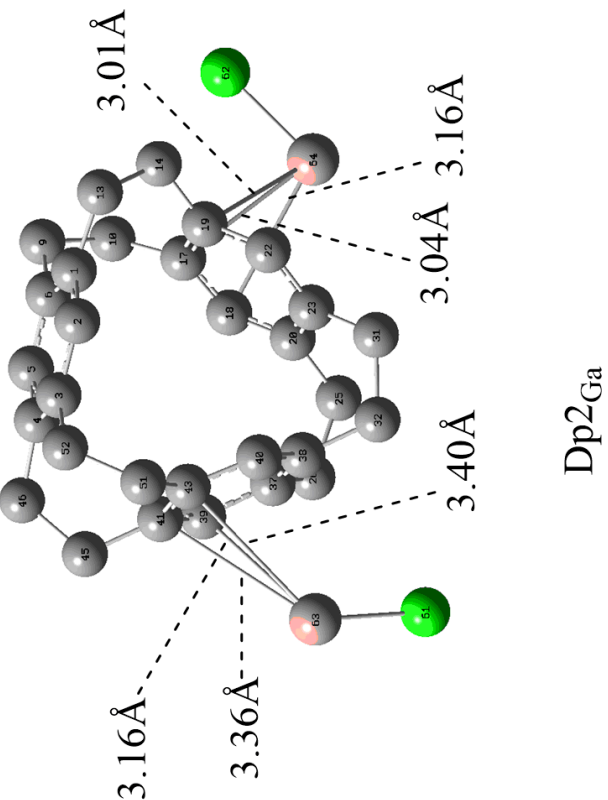

ลี

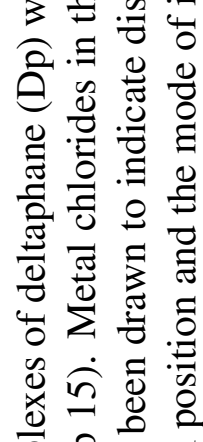

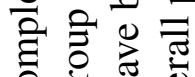

ठํㅝㅇ

훙 ซ

青安造

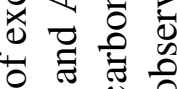

的守

要 离

䗆䲶

光总总

ن 0 过

兽唡。 
Table 3.33: Metal-carbon distances in Dp-indium exclusion complexes. In Dp $1_{\text {In }}$, the M-C bond distances are shown for one top of the cavity carbon atom of each of the three arene rings. $\mathrm{In}^{+}$in $\mathrm{Dp}-\mathrm{In}^{+}$has similar interaction with the three phenyl rings, so bond distances written just for one phenyl ring. Bold text indicates the presence of coordination based on the sum of van der Waal radii that is $\operatorname{In}\left(1.98^{214}\right)+C\left(1.70^{215}\right)=$ $3.68 \AA$.

\begin{tabular}{cccc|ccc|c}
\hline & $D p 1_{\text {In }}$ & \multicolumn{2}{c}{ Dp $2_{\text {In }}$} & \multicolumn{3}{c}{ Dp $3_{\text {In }}$} & \multicolumn{1}{c}{$D p-I^{+}$} \\
\hline & $\mathbf{3 . 4 8}$ & $\mathbf{3 . 1 9}$ & $\mathbf{3 . 1 9}$ & $\mathbf{3 . 2 2}$ & $\mathbf{3 . 2 1}$ & $\mathbf{3 . 2 2}$ & $\mathbf{2 . 8 4}$ \\
& $\mathbf{3 . 5 6}$ & $\mathbf{3 . 2 2}$ & $\mathbf{3 . 2 2}$ & $\mathbf{3 . 2 4}$ & $\mathbf{3 . 2 3}$ & $\mathbf{3 . 2 5}$ & $\mathbf{2 . 8 8}$ \\
& $\mathbf{3 . 6 7}$ & $\mathbf{3 . 3 2}$ & $\mathbf{3 . 3 2}$ & $\mathbf{3 . 3 3}$ & $\mathbf{3 . 3 3}$ & $\mathbf{3 . 3 3}$ & $\mathbf{2 . 9 0}$ \\
& & $\mathbf{3 . 3 4}$ & $\mathbf{3 . 3 4}$ & $\mathbf{3 . 3 5}$ & $\mathbf{3 . 3 6}$ & $\mathbf{3 . 3 5}$ & $\mathbf{2 . 8 4}$ \\
& & $\mathbf{3 . 4 8}$ & $\mathbf{3 . 4 8}$ & $\mathbf{3 . 4 8}$ & $\mathbf{3 . 5 0}$ & $\mathbf{3 . 4 8}$ & $\mathbf{2 . 8 8}$ \\
Coordination & $\eta^{1} \cdot \eta^{1} \cdot \eta^{1}$ & $\mathbf{3 . 5 3}$ & $\mathbf{3 . 5 3}$ & $\mathbf{3 . 5 3}$ & $\mathbf{3 . 5 5}$ & $\mathbf{3 . 5 3}$ & $\mathbf{2 . 9 0}$ \\
\hline
\end{tabular}

Table 3.34: Metal-carbon distances in Dp-thalium exclusion complexes. In Dp1 $1_{\mathrm{Tl}}$, the $\mathrm{M}-\mathrm{C}$ bond distances are shown for one top of the cavity carbon atom of each of the three arene rings. $\mathrm{Tl}^{+}$in $\mathrm{Dp}-\mathrm{Tl}^{+}$has similar interaction with the three phenyl rings, so bond distances written just for one phenyl ring. Bold text indicates the presence of coordination based on the sum of van der Waal radii that is $\mathrm{Tl}\left(2.08^{216}\right)+\mathrm{C}\left(1.70^{215}\right)=$ $3.78 \AA$.

\begin{tabular}{|c|c|c|c|c|c|c|c|}
\hline & $\mathrm{Dp} 1_{\mathrm{Tl}}$ & \multicolumn{2}{|c|}{$\mathrm{Dp} 2_{\mathrm{Tl}}$} & \multicolumn{3}{|c|}{$\mathrm{Dp} 3_{\mathrm{Tl}}$} & $\mathrm{Dp}-\mathrm{Tl}^{+}$ \\
\hline & 3.46 & 3.22 & 3.23 & 3.25 & 3.24 & 3.25 & 2.86 \\
\hline & 3.50 & 3.25 & 3.25 & 3.28 & 3.26 & 3.28 & 2.90 \\
\hline & 3.61 & 3.35 & 3.34 & 3.35 & 3.36 & 3.36 & 2.91 \\
\hline & & 3.38 & 3.38 & 3.38 & 3.39 & 3.38 & 2.86 \\
\hline & & 3.51 & 3.51 & 3.50 & 3.52 & 3.51 & 2.90 \\
\hline & & 3.56 & 3.56 & 3.55 & 3.57 & 3.56 & 2.91 \\
\hline
\end{tabular}

$91^{\circ}$ respectively in un-complexed $\mathrm{Dp}$. The exclusion complexes of the selected Group 14 metals involve $\eta^{2}$ coordination of each metal chloride with respective phenyl ring despite the fact that all M-C distances are within the sum of the van der Waal radii. Here we propose that the metal chloride makes a quasi-tetrahedral coordination with the phenyl ring. Dihedral angles in Group 15 exclusion complexes do not undergo any notable changes. This discussion reveals the rigidity of the Dp moiety which influences the stability of its complexes with metals as evident from highly endothermic enthalpies of formation compared to that of $p \mathrm{Cp}$ complexes. 
Table 3.35: Metal-carbon distances in Dp-germanium exclusion complexes. In Dp1 $1_{\mathrm{Ge}}$, the $\mathrm{M}-\mathrm{C}$ bond distances are shown for one top of the cavity carbon atom of each of the three arene rings. $\mathrm{Ge}^{2+}$ in $\mathrm{Dp}-\mathrm{Ge}^{2+}$ has similar interaction with the three phenyl rings, so bond distances written just for one phenyl ring. Bold text indicates the presence of coordination. The sum of van der Waal radii that is $\mathrm{Ge}\left(2.11^{216}\right)+\mathrm{C}\left(1.70^{215}\right)=3.81$ $\AA$ shows that all the M-C distances are classified as coordination but the visual representation of the complexes shows otherwise. Hence, the classification of coordination is based on three-dimensional view.

\begin{tabular}{cccc|ccc|c}
\hline & $\mathrm{Dp} 1_{\mathrm{Ge}}$ & \multicolumn{2}{c}{$\mathrm{Dp} 2_{\mathrm{Ge}}$} & \multicolumn{3}{c}{$\mathrm{Dp} 3_{\mathrm{Ge}}$} & $\mathrm{Dp}-\mathrm{Ge}^{2+}$ \\
\hline & $\mathbf{3 . 1 2}$ & $\mathbf{2 . 8 2}$ & $\mathbf{2 . 8 1}$ & $\mathbf{2 . 8 3}$ & $\mathbf{2 . 8 4}$ & $\mathbf{2 . 8 4}$ & $\mathbf{2 . 7 5}$ \\
3.42 & $\mathbf{2 . 9 0}$ & $\mathbf{2 . 9 0}$ & $\mathbf{2 . 9 2}$ & $\mathbf{2 . 9 1}$ & $\mathbf{2 . 9 2}$ & $\mathbf{2 . 7 6}$ \\
& 3.45 & 3.05 & 3.05 & 3.06 & 3.08 & 3.07 & $\mathbf{2 . 7 9}$ \\
& & 3.26 & 3.26 & 3.29 & 3.27 & 3.27 & $\mathbf{2 . 7 5}$ \\
& & 3.38 & 3.38 & 3.40 & 3.40 & 3.40 & $\mathbf{2 . 7 6}$ \\
& & 3.52 & 3.53 & 3.54 & 3.53 & 3.53 & $\mathbf{2 . 7 9}$ \\
\hline Coordination & $\eta^{1}$ & $\eta^{2} . \eta^{2}$ & \multicolumn{3}{c}{$\eta^{2} . \eta^{2} . \eta^{2}$} & $\eta^{6} . \eta^{6} \cdot \eta^{6}$ \\
\hline
\end{tabular}

Table 3.36: Metal-carbon distances in Dp-tin exclusion complexes. In Dp1 $1_{\mathrm{Sb}}$, the M-C bond distances are shown for one top of the cavity carbon atom of each of the three arene rings. $\mathrm{Sn}^{2+}$ in $\mathrm{Dp}-\mathrm{Sn}^{2+}$ has similar interaction with the three phenyl rings, so bond distances written just for one phenyl ring. Based on the sum of van der Waal radii that is $\operatorname{Sn}\left(2.17^{213}\right)+\mathrm{C}\left(1.70^{215}\right)=3.87 \AA$ shows that all the M-C distances are classified as coordination but the visual representation of the complexes shows otherwise. Hence, the classification of coordination is based on three-dimensional view.

\begin{tabular}{cccc|ccc|c}
\hline & $\mathrm{Dp} 1_{\mathrm{Sn}}$ & \multicolumn{2}{c}{$\mathrm{Dp} 2_{\mathrm{Sn}}$} & \multicolumn{3}{c}{$\mathrm{Dp} 3_{\mathrm{Sn}}$} & $\mathrm{Dp}-\mathrm{Sn}^{2+}$ \\
\hline & $\mathbf{3 . 1 8}$ & $\mathbf{2 . 9 8}$ & $\mathbf{2 . 9 8}$ & $\mathbf{3 . 0 0}$ & $\mathbf{3 . 0 1}$ & $\mathbf{3 . 0 0}$ & $\mathbf{2 . 8 0}$ \\
3.45 & $\mathbf{3 . 0 3}$ & $\mathbf{3 . 0 3}$ & $\mathbf{3 . 0 5}$ & $\mathbf{3 . 0 4}$ & $\mathbf{3 . 0 4}$ & $\mathbf{2 . 8 2}$ \\
3.49 & 3.19 & 3.19 & 3.21 & 3.22 & 3.22 & $\mathbf{2 . 8 4}$ \\
& & 3.36 & 3.36 & 3.37 & 3.34 & 3.36 & $\mathbf{2 . 8 0}$ \\
& & 3.49 & 3.49 & 3.50 & 3.50 & 3.51 & $\mathbf{2 . 8 2}$ \\
& & 3.61 & 3.61 & 3.63 & 3.60 & 3.62 & $\mathbf{2 . 8 4}$ \\
\hline Coordination & $\eta^{1}$ & $\eta^{2} . \eta^{2}$ & \multicolumn{3}{c}{$\eta^{2} . \eta^{2} \cdot \eta^{2}$} & $\eta^{6} \cdot \eta^{6} \cdot \eta^{6}$ \\
\hline
\end{tabular}

\subsubsection{Inclusion Complexes}

To investigate the possible coordination mode and interaction properties that the main cavity of Dp may have, we optimized the ground state geometries of $\mathrm{Dp} 1_{\mathrm{M}}$ and $\mathrm{Dp}-\mathrm{M}^{\mathrm{n}+}$ inclusion complexes as in the case of $p \mathrm{Cp}$. The trends within a group of metals remains the same, so Figures 3.12 and 3.13 show one example from each group for both types of compounds. In all $\mathrm{Dp} 1_{\mathrm{M}}$ complexes where the metal chloride coordinates from above 
Table 3.37: Metal-carbon distances in Dp-lead exclusion complexes. In Dp1 $1_{\mathrm{Pb}}$, the $\mathrm{M}-\mathrm{C}$ bond distances are shown for one top of the cavity carbon atom of each of the three arene rings. $\mathrm{Pb}^{2+}$ in $\mathrm{Dp}-\mathrm{Pb}^{2+}$ has similar interaction with the three phenyl rings, so bond distances written just for one phenyl ring. Based on the sum of van der Waal radii that is $\mathrm{Pb}\left(2.02^{213}\right)+\mathrm{C}\left(1.70^{215}\right)=3.72 \AA$, shows that all the M-C distances are classified as coordination but the visual representation of the complexes shows otherwise. Hence, the classification of coordination is based on three-dimensional view.

\begin{tabular}{cccc|ccc|c}
\hline & $\mathrm{Dp} 1_{\mathrm{Pb}}$ & \multicolumn{2}{c}{$\mathrm{Dp} 2_{\mathrm{Pb}}$} & \multicolumn{3}{c}{$\mathrm{Dp} 3_{\mathrm{Pb}}$} & $\mathrm{Dp}-\mathrm{Pb}^{2+}$ \\
\hline & $\mathbf{3 . 0 8}$ & $\mathbf{3 . 0 2}$ & $\mathbf{3 . 0 0}$ & $\mathbf{3 . 0 3}$ & $\mathbf{3 . 0 1}$ & $\mathbf{3 . 0 3}$ & $\mathbf{2 . 8 2}$ \\
3.34 & $\mathbf{3 . 0 1}$ & $\mathbf{3 . 0 3}$ & $\mathbf{3 . 0 4}$ & $\mathbf{3 . 0 8}$ & $\mathbf{3 . 0 4}$ & $\mathbf{2 . 8 5}$ \\
& 3.37 & 3.20 & 3.20 & 3.22 & 3.24 & 3.23 & $\mathbf{2 . 8 6}$ \\
& & 3.31 & 3.32 & 3.32 & 3.29 & 3.32 & $\mathbf{2 . 8 2}$ \\
& & 3.46 & 3.47 & 3.47 & 3.49 & 3.49 & $\mathbf{2 . 8 5}$ \\
& & 3.56 & 3.57 & 3.57 & 3.51 & 3.58 & $\mathbf{2 . 8 6}$ \\
\hline Coordination & $\eta^{1}$ & $\eta^{2} \cdot \eta^{2}$ & \multicolumn{3}{c}{$\eta^{2} . \eta^{2} . \eta^{2}$} & $\eta^{6} \cdot \eta^{6} \cdot \eta^{6}$ \\
\hline
\end{tabular}

Table 3.38: Metal-carbon distances in Dp-arsenic exclusion complexes. In $\mathrm{Dp} 1_{\mathrm{As}}$, the M-C bond distances are shown for one top of the cavity carbon atom of each of the three arene rings. $\mathrm{As}^{3+}$ in $\mathrm{Dp}-\mathrm{As}^{3+}$ has similar interaction with the three phenyl rings, so bond distances written just for one phenyl ring. Bold text indicates the presence of coordination based on the sum of van der Waal radii that is As $\left(1.85^{213}\right)+\mathrm{C}\left(1.70^{215}\right)$ $=3.55 \AA$.

\begin{tabular}{cccc|ccc|c}
\hline & $\mathrm{Dp} 1_{\mathrm{As}}$ & \multicolumn{2}{c}{$\mathrm{Dp} 2_{\mathrm{As}}$} & \multicolumn{3}{c}{$\mathrm{Dp} 3_{\mathrm{As}}$} & $\mathrm{Dp}-\mathrm{As}^{3+}$ \\
\hline & $\mathbf{3 . 5 2}$ & $\mathbf{3 . 1 8}$ & $\mathbf{3 . 1 3}$ & $\mathbf{3 . 1 5}$ & $\mathbf{3 . 1 5}$ & $\mathbf{3 . 1 5}$ & $\mathbf{2 . 7 3}$ \\
& $\mathbf{3 . 5 2}$ & $\mathbf{3 . 1 9}$ & $\mathbf{3 . 1 8}$ & $\mathbf{3 . 2 0}$ & $\mathbf{3 . 2 0}$ & $\mathbf{3 . 2 0}$ & $\mathbf{2 . 7 0}$ \\
& $\mathbf{3 . 5 2}$ & $\mathbf{3 . 2 8}$ & $\mathbf{3 . 2 9}$ & $\mathbf{3 . 3 1}$ & $\mathbf{3 . 3 1}$ & $\mathbf{3 . 3 2}$ & $\mathbf{2 . 7 7}$ \\
& & $\mathbf{3 . 3 1}$ & $\mathbf{3 . 3 8}$ & $\mathbf{3 . 4 0}$ & $\mathbf{3 . 4 0}$ & $\mathbf{3 . 4 1}$ & $\mathbf{2 . 7 3}$ \\
& & $\mathbf{3 . 4 0}$ & $\mathbf{3 . 4 1}$ & $\mathbf{3 . 4 2}$ & $\mathbf{3 . 4 2}$ & $\mathbf{3 . 4 4}$ & $\mathbf{2 . 7 0}$ \\
\hline Coordination & $\eta^{1} \cdot \eta^{1} \cdot \eta^{1}$ & $\mathbf{3 . 4 6}$ & $\mathbf{3 . 4 6}$ & $\mathbf{3 . 4 7}$ & $\mathbf{3 . 4 7}$ & $\mathbf{3 . 4 9}$ & $\mathbf{2 . 7 7}$ \\
\hline
\end{tabular}

the Dp cavity, the metal chloride may interact with one carbon from each phenyl ring. Hence, these complexes may involve a maximum of $\eta^{1} \cdot \eta^{1} \cdot \eta^{1}$ coordination of metal with the Dp moiety. Due to the rigidity in un-complexed Dp imposed by the two ethano bridges on either side of each phenyl ring, there is no considerable deformation of the Dp geometry and hence all dihedral angles undergo only minor changes.

Group $13 \mathrm{Dp} 1_{\mathrm{M}}$ complexes are the case where the metal chloride interacts with the three carbon atoms. This gives rise to $\eta^{1} \cdot \eta^{1} \cdot \eta^{1}$ coordination mode. In Group 14 complexes, 
Table 3.39: Metal-carbon distances in Dp-antimony exclusion complexes. In $\mathrm{Dp} 1_{\mathrm{Sb}}$, the $\mathrm{M}-\mathrm{C}$ bond distances are shown for one top of the cavity carbon atom of each of the three arene rings. $\mathrm{Sb}^{3+}$ in $\mathrm{Dp}-\mathrm{Sb}^{3+}$ has similar interaction with the three phenyl rings, so bond distances written just for one phenyl ring. Bold text indicates the presence of coordination based on the sum of van der Waal radii that is $\mathrm{Sb}\left(2.06^{213}\right)+\mathrm{C}\left(1.70^{215}\right)=$ $3.76 \AA$.

\begin{tabular}{cccc|ccc|c}
\hline & $\mathrm{Dp} 1_{\mathrm{Sb}}$ & \multicolumn{2}{c}{$\mathrm{Dp} 2_{\mathrm{Sb}}$} & \multicolumn{3}{c}{$\mathrm{Dp} 3_{\mathrm{Sb}}$} & $\mathrm{Dp}-\mathrm{Sb}^{3+}$ \\
\hline & $\mathbf{3 . 4 0}$ & $\mathbf{3 . 2 2}$ & $\mathbf{3 . 1 8}$ & $\mathbf{3 . 2 5}$ & $\mathbf{3 . 2 0}$ & $\mathbf{3 . 2 0}$ & $\mathbf{2 . 7 7}$ \\
& $\mathbf{3 . 4 0}$ & $\mathbf{3 . 2 3}$ & $\mathbf{3 . 2 4}$ & $\mathbf{3 . 2 5}$ & $\mathbf{3 . 2 6}$ & $\mathbf{3 . 2 6}$ & $\mathbf{2 . 7 7}$ \\
& $\mathbf{3 . 4 0}$ & $\mathbf{3 . 3 4}$ & $\mathbf{3 . 3 6}$ & $\mathbf{3 . 3 6}$ & $\mathbf{3 . 3 8}$ & $\mathbf{3 . 3 8}$ & $\mathbf{2 . 8 1}$ \\
& & $\mathbf{3 . 4 0}$ & $\mathbf{3 . 4 6}$ & $\mathbf{3 . 4 2}$ & $\mathbf{3 . 4 8}$ & $\mathbf{3 . 4 8}$ & $\mathbf{2 . 7 7}$ \\
& & $\mathbf{3 . 5 0}$ & $\mathbf{3 . 5 0}$ & $\mathbf{3 . 5 2}$ & $\mathbf{3 . 5 2}$ & $\mathbf{3 . 5 2}$ & $\mathbf{2 . 7 7}$ \\
Coordination & $\eta^{1} \cdot \eta^{1} \cdot \eta^{1}$ & $\mathbf{3 . 5 7}$ & $\mathbf{3 . 5 6}$ & $\mathbf{3 . 6 0}$ & $\mathbf{3 . 5 8}$ & $\mathbf{3 . 5 7}$ & $\mathbf{2 . 8 1}$ \\
\hline
\end{tabular}

Table 3.40: Metal-carbon distances in Dp-bismuth complexes. In Dp $1_{\mathrm{Bi}}$, the M-C bond distances are shown for one top of the cavity carbon atom of each of the three arene rings. $\mathrm{Bi}^{3+}$ in $\mathrm{Dp}-\mathrm{Bi}^{3+}$ has similar interaction with the three phenyl rings, so bond distances written just for one phenyl ring. Bold text indicates the presence of coordination based on the sum of van der Waal radii that is $\operatorname{Bi}\left(2.07^{213}\right)+C\left(1.70^{215}\right)=3.77 \AA$.

\begin{tabular}{cccc|ccc|c}
\hline & $\mathrm{Dp} 1_{\mathrm{Bi}}$ & \multicolumn{2}{c}{$\mathrm{Dp} 2_{\mathrm{Bi}}$} & \multicolumn{3}{c}{$\mathrm{Dp} 3_{\mathrm{Bi}}$} & $\mathrm{Dp}-\mathrm{Bi}^{3+}$ \\
\hline & $\mathbf{3 . 3 0}$ & $\mathbf{3 . 1 7}$ & $\mathbf{3 . 2 0}$ & $\mathbf{3 . 2 2}$ & $\mathbf{3 . 1 9}$ & $\mathbf{3 . 2 0}$ & $\mathbf{2 . 7 9}$ \\
& $\mathbf{3 . 3 0}$ & $\mathbf{3 . 2 2}$ & $\mathbf{3 . 2 3}$ & $\mathbf{3 . 2 6}$ & $\mathbf{3 . 2 4}$ & $\mathbf{3 . 2 4}$ & $\mathbf{2 . 8 0}$ \\
& $\mathbf{3 . 3 0}$ & $\mathbf{3 . 3 2}$ & $\mathbf{3 . 3 0}$ & $\mathbf{3 . 3 3}$ & $\mathbf{3 . 3 4}$ & $\mathbf{3 . 3 4}$ & $\mathbf{2 . 8 2}$ \\
& & $\mathbf{3 . 3 9}$ & $\mathbf{3 . 3 2}$ & $\mathbf{3 . 4 1}$ & $\mathbf{3 . 4 4}$ & $\mathbf{3 . 4 2}$ & $\mathbf{2 . 7 9}$ \\
& & $\mathbf{3 . 4 1}$ & $\mathbf{3 . 4 1}$ & $\mathbf{3 . 4 4}$ & $\mathbf{3 . 4 2}$ & $\mathbf{3 . 4 4}$ & $\mathbf{2 . 8 0}$ \\
Coordination & $\eta^{1} . \eta^{1} . \eta^{1}$ & $\mathbf{3 . 4 6}$ & $\mathbf{3 . 4 6}$ & $\mathbf{3 . 4 8}$ & 3.49 & $\mathbf{3 . 4 8}$ & $\mathbf{2 . 8 2}$ \\
\hline
\end{tabular}

the range of metal-carbon distances increases significantly giving rise to $\eta^{1}$ coordination. For example, in $\mathrm{Dp} 1_{\mathrm{Ge}}$, metal is $3.12 \AA$ away from one peripheral carbon while the other two distances are $3.42 \AA$ and $3.45 \AA$. The metal chlorides in Group $15 \mathrm{Dp} 1_{\mathrm{M}}$ are essentially $\eta^{1} \cdot \eta^{1} \cdot \eta^{1}$-coordinated as suggested by the metal-carbon distances. For instance, $\mathrm{AsCl}_{3}$ in $\mathrm{Dp}_{\mathrm{As}}$ is equidistant from all three carbon atoms with a metal-carbon distance of $3.52 \AA$.

All the $\mathrm{Dp}-\mathrm{M}^{\mathrm{n}+}$ inclusion complexes with $\mathrm{D}_{3}$ have $\eta^{6} \cdot \eta^{6} \cdot \eta^{6}$ coordination of the metal cation inside the Dp cavity. The ground state minima of these complexes were possible 
to optimize at $\mathrm{D}_{3}$ as well as $\mathrm{C}_{3}$ symmetry. There are no notable deviations of geometry when compared to the un-complexed Dp.

While all groups have the same coordination mode in the case of $\mathrm{Dp}-\mathrm{M}^{\mathrm{n}+}$ complexes, there is a trend of increasing metal-carbon distances down the group which can be simply attributed to the increasing size of metal cations. The same concept may be applied to explain comparatively shorter distances in Group 15 complexes where Dp$\mathrm{As}^{3+}$ demonstrates the strongest interaction among the nine complexes of its kind.

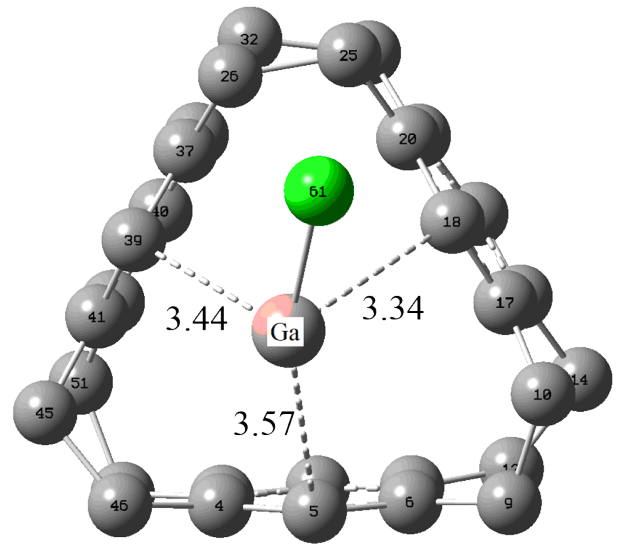

$\mathrm{Dp}_{\mathrm{Ga}}$

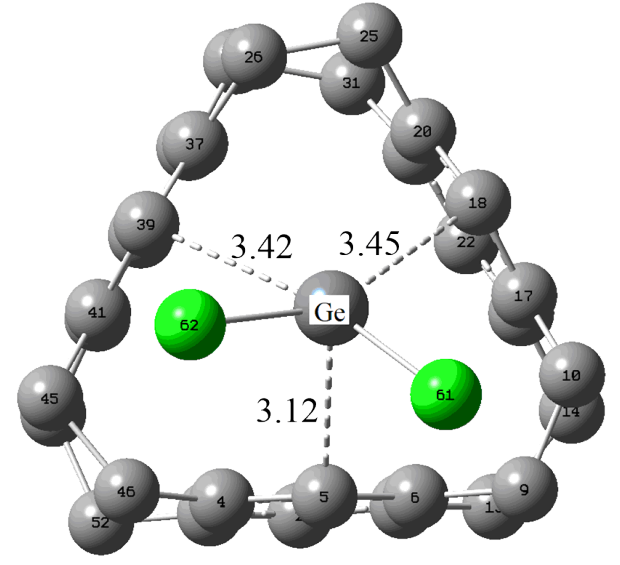

$\mathrm{Dp}_{\mathrm{Ge}}$

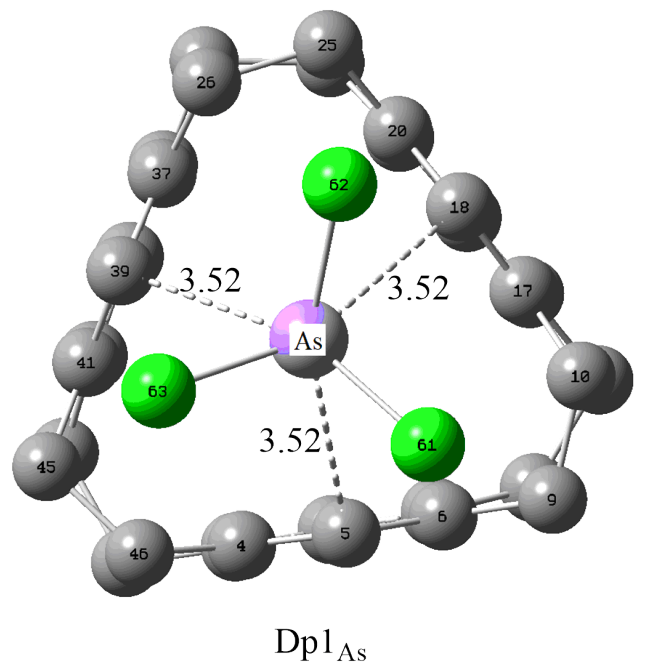

Figure 3.12: Structures of the inclusion complexes of deltaphane (Dp) with metal chlorides forming $\mathrm{Dp}\left(\mathrm{MCl}_{\mathrm{n}}\right)$ denoted as $\mathrm{Dp} 1_{\mathrm{M}}$ optimized at PBE0-D3BJ/def2TZVP level. $M$ represents $\mathrm{Ga}$ (for Group 13), Ge (for Group 14) and As (for Group 15). In Dp1 $1_{\mathrm{M}}$, metal chlorides coordinate with the outer peripheries of the phenyl rings of $\mathrm{Dp}$ from above the cavity. It is to be noted that metal-carbon bonds have been drawn to indicate distances. 


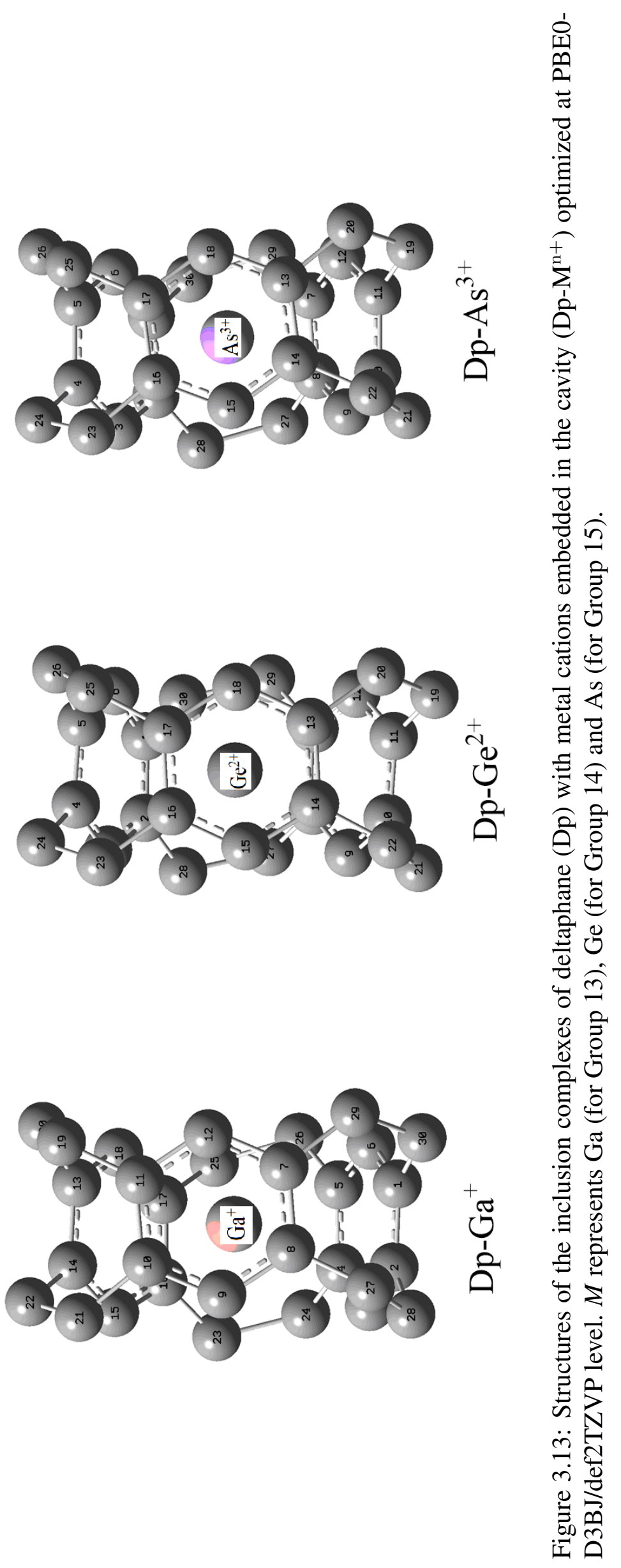




\subsubsection{Thermodynamic Properties}

As for $p \mathrm{Cp}$ complexes, we calculated the enthalpies of formation for all Dp complexes (Table 3.41). While moving down a group of metals, the exothermic formation of the exclusion complexes is gradually more favoured despite some exceptions. If we draw a comparison among the three groups, the formation of Dp exclusion complexes (with $\eta^{2}$ coordination of metal chloride with phenyl ring), coordination is the most favoured in case of Group 14. Here, the metal chloride is assumed to form a quasi-tetrahedral complex with the two atoms of a phenyl ring (considered as one discrete unit) below the plane, the metal atom on the plane and the two chlorides and the lone pair of the metal atom lying above the plane. While comparing $\mathrm{Dp} 3_{\mathrm{M}}$ to $\mathrm{Dp} 2_{\mathrm{M}}$ the formation of the former is more exothermic due to comparatively more even $\pi$ charge donation from the outer surface of the cavity.

Table 3.41: Enthalpies $(\mathrm{kcal} / \mathrm{mol})$ of formation of metal complexes with deltaphane $(\mathrm{Dp})$ in the reaction $\mathrm{Dp}+\mathrm{NMCl}_{n} \rightarrow \mathrm{Dp}\left(\mathrm{MCl}_{\mathrm{n}}\right) \mathrm{N}$. Each exclusion complex is represented by $\mathrm{DpN}_{\mathrm{M}}$ where $\mathrm{N}=1,2$ or 3 for $\mathrm{Dp}\left(\mathrm{MCl}_{\mathrm{n}}\right), \mathrm{Dp}\left(\mathrm{MCl}_{\mathrm{n}}\right)_{2}, \mathrm{Dp}\left(\mathrm{MCl}_{\mathrm{n}}\right)_{3}$ respectively and $\mathrm{M}=\mathrm{Ga}, \mathrm{In}, \mathrm{Tl}, \mathrm{Ge}, \mathrm{Sn}, \mathrm{Pb}, \mathrm{As}, \mathrm{Sb}$ and $\mathrm{Bi}$. $n$ represents the number of chlorides attached to the metal in each case and its value is 1,2 and 3 for the metals of Group 13, 14 and 15 respectively. The inclusion complexes Dp- $\mathrm{M}_{\mathrm{n}}{ }^{+}$contain $\mathrm{M}_{\mathrm{n}}{ }^{+}$in the centre of the cavity of Dp. They are formed as $\mathrm{Dp}+\mathrm{MCl}_{\mathrm{n}} \rightarrow \mathrm{Dp}-\mathrm{M}_{\mathrm{n}}^{+}+\mathrm{nCl}^{-}$where $n$ denotes 1,2, and 3 for the metals of Group 13, 14 and 15 respectively.

\begin{tabular}{lcccccccc}
\hline & $\mathrm{Dp} 1_{\mathrm{M}}$ & $\Delta \mathrm{H}_{f}$ & $\mathrm{Dp} 2_{\mathrm{M}}$ & $\Delta \mathrm{H}_{f}$ & $\mathrm{Dp} 3_{\mathrm{M}}$ & $\Delta \mathrm{H}_{f}$ & $\mathrm{Dp}-\mathrm{M}^{\mathrm{n}+}$ & $\Delta \mathrm{H}_{f}$ \\
\hline \multirow{4}{*}{ Group 13 } & $\mathrm{Dp}_{\mathrm{Ga}}$ & -7.7 & $\mathrm{Dp} 2_{\mathrm{Ga}}$ & -23.5 & $\mathrm{Dp} 3_{\mathrm{Ga}}$ & -30.0 & $\mathrm{Dp}-\mathrm{Ga}^{+}$ & 38.9 \\
& $\mathrm{Dp} 1_{\mathrm{In}}$ & -8.9 & $\mathrm{Dp} 2_{\mathrm{In}}$ & -25.7 & $\mathrm{Dp} 3_{\mathrm{In}}$ & -35.5 & $\mathrm{Dp}-\mathrm{In}^{+}$ & 52.9 \\
& $\mathrm{Dp} 1_{\mathrm{Tl}}$ & -10.0 & $\mathrm{Dp} 2_{\mathrm{Tl}}$ & -27.1 & $\mathrm{Dp} 3_{\mathrm{Tl}}$ & -37.4 & $\mathrm{Dp}-\mathrm{Tl}^{+}$ & 52.3 \\
\hline \multirow{3}{*}{ Group 14 } & $\mathrm{Dp} 1_{\mathrm{Ge}}$ & -8.7 & $\mathrm{Dp} 2_{\mathrm{Ge}}$ & -29.8 & $\mathrm{Dp} 3_{\mathrm{Ge}}$ & -38.0 & $\mathrm{Dp}-\mathrm{Ge}^{2+}$ & 167.8 \\
& $\mathrm{Dp} 1_{\mathrm{Sn}}$ & -10.8 & $\mathrm{Dp} 2_{\mathrm{Sn}}$ & -34.2 & $\mathrm{Dp} 3_{\mathrm{Sn}}$ & -47.2 & $\mathrm{Dp}-\mathrm{Sn}^{2+}$ & 168.0 \\
& $\mathrm{Dp} 1_{\mathrm{Pb}}$ & -13.5 & $\mathrm{Dp} 2_{\mathrm{Pb}}$ & -37.3 & $\mathrm{Dp} 3_{\mathrm{Pb}}$ & -51.7 & $\mathrm{Dp}-\mathrm{Pb}^{2+}$ & 162.5 \\
\hline \multirow{3}{*}{ Group 15 } & $\mathrm{Dp} 1_{\mathrm{As}}$ & -9.1 & $\mathrm{Dp} 2_{\mathrm{As}}$ & -20.3 & $\mathrm{Dp} 3_{\mathrm{As}}$ & -23.8 & $\mathrm{Dp}-\mathrm{As}^{3+}$ & 416.5 \\
& $\mathrm{Dp} 1_{\mathrm{Sb}}$ & -11.2 & $\mathrm{Dp} 2_{\mathrm{Sb}}$ & -26.0 & $\mathrm{Dp} 3_{\mathrm{Sb}}$ & -34.4 & $\mathrm{Dp}-\mathrm{Sb}^{3+}$ & 400.0 \\
& $\mathrm{Dp} 1_{\mathrm{Bi}}$ & -15.6 & $\mathrm{Dp} 2_{\mathrm{Bi}}$ & -32.8 & $\mathrm{Dp} 3_{\mathrm{Bi}}$ & -43.7 & $\mathrm{Dp}-\mathrm{Bi}^{3+}$ & 390.0 \\
\hline
\end{tabular}

All Dp1 $1_{M}$ inclusion complexes have almost the same thermodynamic feasibility. If we see this in connection to the large but almost identical metal-carbon distances, it can be inferred that it is the rigidity of the Dp ring that does not allow it to accommodate the metal chlorides closer than $3.00 \AA$. 
$\Delta \mathrm{H}_{f}$ of $\mathrm{Dp}-\mathrm{M}^{\mathrm{n}+}$ shows a complicated trend as their formation is affected by a number of factors. Since the formation of each of these complexes involves the dissociation of the respective metal chloride, the reaction becomes more endothermic while moving from one group to the next. Group $13 \mathrm{MCl}$ involves a single energy of dissociation compared to the additional second, in the case of Group $14 \mathrm{MCl}_{2}$ and, in Group 15 $\mathrm{MCl}_{3}$, a third energy of dissociation. Hence the formation of Group $13 \mathrm{Dp}-\mathrm{M}^{\mathrm{n}+}$ is thermodynamically least disfavoured. In Group 13 complexes the smallest metal-carbon distances are in case of $\mathrm{Ga}^{+}$and this also has the least endothermic formation. The trends within Group 13 are, however, opposite to the other groups. While the formation of these complexes tends to be more exothermic down the group in Group 15, Dp- $\mathrm{Ga}^{+}$ from Group 13 presents the case with least endothermic formation. The difference in these trends will be evaluated on the basis of MZEDA below. The formation of Dp$\mathrm{Ge}^{2+}$ and $\mathrm{Dp}-\mathrm{Sn}^{2+}$ can be considered to be equally endothermic compared to that of $\mathrm{Dp}-\mathrm{Pb}^{2+}$.

\subsubsection{MZEDA of the Dp-M $\mathrm{M}^{\mathrm{n}+}$ Complexes}

The trends in the results of energy decomposition analysis of $\mathrm{Dp}-\mathrm{M}^{\mathrm{n}+}$ complexes do not differ from the case of $p C p-\mathrm{M}^{\mathrm{n}+}$ except the fact that unlike other Dp- $\mathrm{M}^{\mathrm{n}+}$ complexes and all the $p C p-\mathrm{M}^{\mathrm{n}+}$ complexes the trends of $\Delta \mathrm{H}_{f}$ in Group $13 \mathrm{Dp}-\mathrm{M}^{n+}$ are consistent with these results. While in all other cases $\Delta \mathrm{H}_{f}$ of the complexes seems dependent on the $\Delta \mathrm{H}_{\text {dissoc }}$ of the corresponding metal chloride, the former is the lowest for $\mathrm{Dp}-\mathrm{Ga}^{+}$ among the Group $13 \mathrm{Dp}$ complexes despite the highest $\Delta \mathrm{H}_{\text {dissoc }}$ of $\mathrm{GaCl}$ among the Group 13 metal chlorides. Here, MZEDA (Table 3.42) seems to solve the puzzle where we can see that $\Delta E^{\text {int }}$ of Dp-Ga ${ }^{+}(-60.52 \mathrm{kcal} / \mathrm{mol})$ is $25.0 \mathrm{kcal} / \mathrm{mol}$ and $24.5 \mathrm{kcal} / \mathrm{mol}$ stronger than that of $\mathrm{Dp}-\mathrm{In}^{+}(-35.52 \mathrm{kcal} / \mathrm{mol})$ and $\mathrm{Dp}-\mathrm{Tl}^{+}(-28.78 \mathrm{kcal} / \mathrm{mol})$. The ratio of $\Delta E^{\text {orb }}$ to $\Delta E^{\text {ele }}$ for $\mathrm{Dp}-\mathrm{Ga}^{+}$is 1.2 while it is 0.8 for each of $\mathrm{Dp}-\mathrm{In}^{+}$and $\mathrm{Dp}-\mathrm{Tl}^{+}$. The metal-ligand distances also support this which are the least for Dp- $\mathrm{Ga}^{+}(2.8 \AA)$ compared to Dp- $\mathrm{In}^{+}(2.8-2.9 \AA)$ and Dp- $\mathrm{Tl}^{+}(2.9 \AA)$. It can be argued that all these factors 
are additive in the formation of $\mathrm{Dp}-\mathrm{Ga}^{+}$with the most exothermic $\Delta \mathrm{H}_{f}$. However, the $\mathrm{C}_{3}$ symmetric minima of Group $13 \mathrm{Dp}-\mathrm{M}^{+}$(EDA values given in parentheses in Table 3.42) show almost the same trends as for their $p \mathrm{Cp}-\mathrm{M}^{+}$counterparts.

The $\mathrm{D}_{3}$ symmetric Dp- $\mathrm{M}^{2+}$ complexes of Group 14 witness stronger coordination compared to that in the ( $\mathrm{D}_{3}$ symmetric transition structure) complexes of Group 13 metals. The ratio of $\Delta E^{\text {orb }}$ to $\Delta E^{\text {ele }}$ is $2.6,2.0$ and 1.8 for $\mathrm{Dp}-\mathrm{Ge}^{2+}, \mathrm{Dp}-\mathrm{Sn}^{2+}$ and $\mathrm{Dp}-\mathrm{Pb}^{2+}$ respectively which shows the strongest coordination is on top of the group (Table 3.43). The values of $\Delta E^{\text {int }}$ are also consistent with the metal-ligand distances i.e. 2.75-2.79 $\AA, 2.80-2.84 \AA$ and 2.82-2.86 $\AA$ respectively. In the case of $\mathrm{C}_{3}$ symmetric minima, the trends match that of the complexes with $\mathrm{D}_{3}$ symmetry. However, all metal cations in the $\mathrm{C}_{3}$ complexes tend to come out of the cavity towards the top. This is then overcompensated by the lower values of $\Delta E^{\text {int }}$ for each $\mathrm{C}_{3}$ symmetric case.

Table 3.42: Results of the MZEDA analysis for M-Deltaphane $\left(\mathrm{M}=\mathrm{Ga}^{+}, \mathrm{In}^{+}, \mathrm{Tl}^{+}\right)$ complexes in $\mathrm{D}_{3}$ symmetry (results for the $\mathrm{C}_{3}$ minimum structures in parentheses) at the PBE0/TZ2P level. The percentage shows the contribution of an energy term in the total attraction energy which is the sum of $\Delta E^{\text {ele }}$ and $\Delta E^{\text {orb }} . \Delta E^{\text {hybrid }}$ is the component of $\Delta E^{\text {orb }}$ expressed separately in the MZEDA results. $A_{1}, A_{2}$ and $E_{1}$ are the non-reducible terms in which $\Delta E^{\text {orb }}$ of a $\mathrm{D}_{3}$ structure can be decomposed. All values in $\mathrm{kcal} \mathrm{mol}^{-1}$.

\begin{tabular}{lcccccc} 
& \multicolumn{2}{c}{ Ga } & \multicolumn{2}{c}{$\mathrm{In}$} & \multicolumn{2}{c}{$\mathrm{Tl}$} \\
\hline$\Delta E^{\text {Pauli }}$ & 105.14 & $(72.51)$ & 149.89 & $(57.30)$ & 149.34 & $(51.92)$ \\
$\Delta E^{\text {ele }}$ & -80.18 & $(-48.98)$ & -102.52 & $(-39.82)$ & -99.57 & $(-36.36)$ \\
& $48.4 \%$ & & $55.4 \%$ & & $55.9 \%$ & \\
$\Delta E^{\text {orb }}$ & -85.48 & $(-75.66)$ & -82.92 & $(-58.98)$ & -78.54 & $(-51.59)$ \\
$\Delta E^{\text {hybrid }}$ & $51.6 \%$ & & $44.6 \%$ & & $44.1 \%$ & \\
\hline$A_{1}$ & 21.25 & $(29.33)$ & 31.98 & $(34.43)$ & 35.67 & $(33.07)$ \\
$A_{2}$ & -7.79 & & -9.08 & & -8.58 & \\
$E_{1}$ & -20.45 & & -19.03 & & -18.84 & \\
\hline$\Delta E^{\text {int }}$ & -57.24 & & -54.81 & & -51.12 & \\
\hline
\end{tabular}

Table 3.44 shows the MZEDA results for Group $15 \mathrm{Dp}-\mathrm{M}^{3+}$ complexes. We observed that the complexes with $\mathrm{D}_{3}$ symmetry in this case possess the strongest interactions among all the deltaphane inclusion complexes. The strength of orbital interaction for Dp-As ${ }^{3+}\left(\Delta E^{\text {orb }}=-633.37 \mathrm{kcal} / \mathrm{mol}\right)$ is not only highest in Group 15 rather it is the 
highest of all the nine complexes of its type. Its strongest $E^{\text {int }}$ of $-666.07 \mathrm{kcal} / \mathrm{mol}$ compared to that of $\mathrm{Dp}-\mathrm{Sb}^{3+}(-542.09 \mathrm{kcal} / \mathrm{mol})$ and $\mathrm{Dp}-\mathrm{Bi}^{3+}(-476.28 \mathrm{kcal} / \mathrm{mol})$ also suggests the same. The ratio of $\Delta E^{\text {orb }}$ to $\Delta E^{\text {ele }}$ is $4.8,3.6$ and 3.3 respectively which implies that all the Group $15 \mathrm{Dp}-\mathrm{M}^{3+}$ bear overall stronger orbital interactions compared to the electrostatic interactions.

Table 3.43: Results of the MZ-EDA analysis for M-Deltaphane $\left(\mathrm{M}=\mathrm{Ge}^{2+}, \mathrm{Sn}^{2+}, \mathrm{Pb}^{2+}\right)$ complexes in $\mathrm{D}_{3}$ symmetry (results for the $\mathrm{C}_{3}$ minimum structures in parentheses) at the PBE0/TZ2P level. The percentage shows the contribution of an energy term in the total attraction energy which is the sum of $\Delta E^{\text {ele }}$ and $\Delta E^{\text {orb }} . \Delta E^{\text {hybrid }}$ is the component of $\Delta E^{\mathrm{orb}}$ expressed separately in the MZEDA results. $A_{1}, A_{2}$ and $E_{1}$ are the non-reducible terms in which $\Delta E^{\text {orb }}$ of a $\mathrm{D}_{3}$ structure can be decomposed. All values in $\mathrm{kcal} \mathrm{mol}^{-1}$.

\begin{tabular}{lcccccc} 
& \multicolumn{2}{c}{$\mathrm{Ge}$} & \multicolumn{2}{c}{$\mathrm{Sn}$} & \multicolumn{2}{c}{$\mathrm{Pb}$} \\
\hline$\Delta E^{\text {Pauli }}$ & 100.36 & $(126.50)$ & 140.15 & $(130.24)$ & 146.76 & $(120.82)$ \\
$\Delta E^{\text {ele }}$ & -102.48 & $(-90.84)$ & -119.12 & $(-94.34)$ & -121.11 & $(-90.38)$ \\
& $27.6 \%$ & & $33.4 \%$ & & $56 \%$ & \\
$\Delta E^{\text {orb }}$ & -268.68 & $(-283.79)$ & -237.98 & $(-235.22)$ & -223.23 & $(-210.59)$ \\
$\Delta E^{\text {hybrid }}$ & $72.4 \%$ & & $66.6 \%$ & & $44 \%$ & \\
\hline$A_{1}$ & -28.49 & $(-1.73)$ & -23.05 & $(11.74)$ & -20.62 & $(15.55)$ \\
$A_{2}$ & -14.21 & & -15.62 & & -15.20 & \\
$E_{1}$ & -71.61 & & -59.95 & & -56.71 & \\
\hline$\Delta E^{\text {int }}$ & -182.86 & & -162.41 & & -151.32 & \\
\hline
\end{tabular}

Table 3.44: Results of the MZEDA analysis for M-Deltaphane $\left(\mathrm{M}=\mathrm{As}^{3+}, \mathrm{Sb}^{3+}, \mathrm{Bi}^{3+}\right)$ complexes in $\mathrm{D}_{3}$ symmetry (The results for the $\mathrm{C}_{3}$ symmetric minimum are given in parentheses) at the PBE0/TZ2P level. The percentage shows the contribution of an energy term in the total attraction energy which is the sum of $\Delta E^{\text {ele }}$ and $\Delta E^{\text {orb }} . \Delta E^{\text {hybrid }}$ is the component of $\Delta E^{\text {orb }}$ expressed separately in the MZEDA results. $A_{1}, A_{2}$ and $E_{1}$ are the non-reducible terms in which $\Delta E^{\text {orb }}$ of a $\mathrm{D}_{3}$ structure can be decomposed. All values in $\mathrm{kcal} \mathrm{mol}^{-1}$.

\begin{tabular}{lcccccc} 
& \multicolumn{2}{c}{ As } & \multicolumn{2}{c}{$\mathrm{Sb}$} & \multicolumn{2}{c}{$\mathrm{Bi}$} \\
\hline$\Delta E^{\text {Pauli }}$ & 100.30 & $(201.07)$ & 140.55 & $(186.07)$ & 154.08 & $(168.78)$ \\
$\Delta E^{\text {ele }}$ & -132.95 & $(-142.09)$ & -147.66 & $(-140.83)$ & -152.61 & $(-134.77)$ \\
& $17.4 \%$ & & $21.6 \%$ & & $23.5 \%$ & \\
$\Delta E^{\text {orb }}$ & -633.37 & $(-734.81)$ & -535.01 & $(-575.48)$ & -496.03 & $(-510.29)$ \\
$\Delta E^{\text {hybrid }}$ & $82.6 \%$ & & $78.4 \%$ & & $76.5 \%$ & \\
\hline$A_{1}$ & -79.90 & $(-77.18)$ & -70.59 & $(-33.34)$ & -70.16 & $(-19.64)$ \\
$A_{2}$ & -26.71 & & -28.93 & & -29.30 & \\
$E_{1}$ & -182.86 & & -144.46 & & -133.37 & \\
\hline$\Delta E^{\text {int }}$ & -423.80 & & -361.63 & & -333.36 & \\
\hline
\end{tabular}




\subsection{Comparison of $p \mathrm{Cp}$ and Dp Complexes}

We have seen that, while both $p \mathrm{Cp}$ and $\mathrm{Dp}$ have $\pi$-rich cavities hosting the main-group metals inside the cavity with higher coordination numbers in most of the cases compared to that from outside the cavity, both types of cyclophanes have different geometries which force them to behave differently in complex formation. In all the cases, $p \mathrm{Cp}$ is expected to accommodate metal chlorides (in $3_{\mathrm{M}}, 2_{\mathrm{M}}$ and $1_{\mathrm{M}}$ complexes) or metal cations (in $p \mathrm{Cp} / \mathrm{Dp}-\mathrm{M}^{\mathrm{n}+}$ complexes) more efficiently due to its more flexible geometry. It readily undergoes the changes in dihedral angles across the ethano bridges and phenyl rings tend to adjust accordingly to coordinate with the $\pi$ acceptor. This increases the thermodynamic feasibility of the $p \mathrm{Cp}$ complexes. On the other hand, the phenyl rings in Dp are locked with two ethano bridges (ortho to one another) on either side and have less freedom of deviation from the geometry of the un-complexed structure. Hence, $\mathrm{Dp}$ is in principle less efficient in forming coordination complexes and the formation of complexes tends to be more endothermic. A comparison of $\Delta \mathrm{H}_{f}$ of $p \mathrm{Cp}$ complexes (Table 3.26) with that of Dp complexes (Table 3.41) show that each $p C p$ complex is thermodynamically more feasible compared to the respective Dp complex.

A comparison of EDA is not so simple. The Group $13 \mathrm{C}_{3}$ symmetric $p \mathrm{Cp}-\mathrm{M}^{\mathrm{n}+}$ complexes show stronger interactions compared to the respective Dp- $\mathrm{M}^{n+}$ complexes and this in line with comparatively exothermic formation of $p \mathrm{Cp}-\mathrm{M}^{\mathrm{n}+}$ complexes. However, Group 14 and $15 \mathrm{Dp}-\mathrm{M}^{\mathrm{n}+}$ complexes exhibit stronger interactions than their $p \mathrm{Cp}$ counterparts while the formation of $p \mathrm{Cp}$ complexes is exothermic compared to their Dp counterparts. This anomaly can be attributed to the thermal effects which arise due to the enhanced vibrational changes upon $p \mathrm{Cp}$ coordination with the cations of higher molecular mass due to the flexible structure of $p \mathrm{Cp}$ compared to the more rigid Dp. Thermal effects are, however, not taken into account while evaluating the interaction energy which comes from the electronic effects of the two frozen fragments. Hence, Group 14 and $15 p$ Cp complexes even with exothermic formation exhibit smaller interaction energy compared to their Dp counterparts. 


\subsection{Conclusion}

The structure and coordination modes of exclusion and inclusion complexes of cyclophanes were investigated through quantum chemical calculations. We explored different coordination possibilities of nine heavier main-group metals with with the inside and outside of $p \mathrm{Cp}$ and Dp cavities. The metal in the Group 14 exclusion complexes and $1_{\mathrm{M}}$ inclusion complexes tend to shift away from the centre of the phenyl ring in the former case or from the centre of the cavity in the latter case. We assume that each of these metals prefers making a quasi-tetrahedral complex one or more sides of the ligand thus attaining stability. Group 13 and 15 exclusion complexes show $\eta^{6} \cdot \eta^{6} \cdot \eta^{6}$ coordination of the metal chloride with the phenyl ring. The mode of attachment of metal chloride with the top of the cyclophane cavity in $\left(p \mathrm{Cp} 1_{\mathrm{M}}\right)$ complexes of Group 13 is different from each other. $\mathrm{GaCl}$ in $p \mathrm{Cp} 1_{\mathrm{Ga}}$ and $\mathrm{TlCl}$ in $p \mathrm{Cp} 1_{\mathrm{Tl}}$ prefer to remain closer to just one carbon while $p \mathrm{Cp} 1_{\text {In }}$ having a comparatively narrower $\mathrm{M}-\mathrm{C}$ range but based on the sum of van der Waal radii, $p \mathrm{Cp} 1_{\mathrm{In}}$ and $p \mathrm{Cp} 1_{\mathrm{Tl}}$ have $\eta^{2} \cdot \eta^{2} \cdot \eta^{2}$ coordination while $p \mathrm{Cp} 1_{\mathrm{Ga}}$ has $\eta^{2} \cdot \eta^{2} \cdot \eta^{1}$ coordination. All the $p \mathrm{Cp} 1_{\mathrm{M}}$ Group 15 complexes also exhibit $\eta^{2} \cdot \eta^{2} \cdot \eta^{2}$ coordination. The $\mathrm{Dp} 1_{\mathrm{M}}$ complexes of Group 13 and 15 are the cases where metal chlorides show $\eta^{1} \cdot \eta^{1} \cdot \eta^{1}$ coordination. In Dp1 $1_{\mathrm{M}}$ complexes of Group 14, we again propose the formation of a quasi-tetrahedral coordination of metal chloride with one out of three top carbon atoms, hence, preferring $\eta^{1}$ coordination.

All the $p \mathrm{Cp} / \mathrm{Dp}-\mathrm{M}^{\mathrm{n}+}$ inclusion complexes with $\mathrm{D}_{3}$ symmetry involve $\eta^{6} \cdot \eta^{6} \cdot \eta^{6}$ coordination of the metal cation inside the cyclophane cavity. In $\mathrm{C}_{3}$ symmetric minima, usually the metal cation tends to come out of the cavity toward the peripheries. Interestingly however, $p \mathrm{Cp}-\mathrm{Ga}^{+}$and $\mathrm{Dp}-\mathrm{Ga}^{+}$are the cases where $\mathrm{Ga}^{+}$even in $\mathrm{C}_{3}$ symmetric geometry prefers essentially $\eta^{6} \cdot \eta^{6} \cdot \eta^{6}$ coordination. As ${ }^{3+}$ shows $\eta^{2} \cdot \eta^{2} \cdot \eta^{2}$ coordination in $p \mathrm{Cp}-\mathrm{As}^{3+}$ with $\mathrm{C}_{3}$ symmetry. The $\mathrm{C}_{3}$ symmetric minima of $p \mathrm{Cp}-\mathrm{In}^{+}$and $p \mathrm{Cp}-\mathrm{Tl}^{+}$have lower interaction energy than $p \mathrm{Cp}-\mathrm{Ga}^{+}$which is not because of higher steric repulsion rather it is due to the comparatively smaller contribution of $\Delta E^{\text {orb }}$ in case of $p \mathrm{Cp}-\mathrm{In}^{+}$ and $p \mathrm{Cp}-\mathrm{Tl}^{+}$. A comparison of MZEDA across the period shows increasing $\Delta E^{\mathrm{orb}}$ 
and the reverse is true for $\Delta E^{\text {ele }}$. This can be attributed to the decreasing cationic size along a period that facilitates $\pi$-charge donation from the cyclophane cavity to the metal cation. Increasing $\Delta E^{\text {orb }}$ in turn increases an overall interaction energy adding to the strength of coordination from left to right in a period.

We propose, based on the different modes of coordination, that inclusion complexes are comparatively more favoured for the synthetic purposes. They all exhibit a perfect $\eta^{6} \cdot \eta^{6} \cdot \eta^{6}$ coordination with most of them having $\mathrm{D}_{3}$ symmetry except the $\mathrm{C}_{3}$ symmetric Group $13 p \mathrm{Cp}-\mathrm{M}^{+}$and $p \mathrm{Cp}-\mathrm{As}^{3+}$ complexes. Moreover based on thermodynamic feasibility, $p \mathrm{Cp}$-metal inclusion complexes are comparatively more favoured than their Dp analogues. 


\section{Chapter 4}

\section{A Computational Study of Structure and Bonding Properties of a}

\section{Heterobimetallic Indium-Zinc}

\section{Compound with Mesityl Azide}

Chapter 1 describes how main-group metals have applications in various types of reactions. There is an interest in exploring the synthesis and reactivity of heterobimetallic compounds of main-group metals due to their versatile applications in catalysis, materials and in synthesizing new reagents. ${ }^{136,137}$ There are known examples of these complexes where a metal-metal bond exists through a donor-acceptor interaction, for example ${ }^{138} \mathrm{Ga}-\mathrm{Ni}$ and In-Ni. Examples are also known with Ga-M' (M' $=\mathrm{Na}, \mathrm{Zn}$ and Cd) bonds. ${ }^{141}$ where gallium acts as a donor. Inspired by these developments the exploration of the reactivity of these compounds is being carried out in the research group of Professor Coles at Victoria University of Wellington. They first synthesized a heterobimetallic compound with In-Zn bond and further carried out an insertion reaction where a mesityl amine gets inserted across the In- $\mathrm{Zn}$ forming In- $\mathrm{N}$ and $\mathrm{Zn}-\mathrm{N}$ bonds. We wished to gain a computational insight into the structure and bonding properties 
of these newly synthesized compounds. The calculations in this part utilize PBE0D3BJ/def2SVP method.

\subsection{Investigation of Structural and Bonding Properties of Heterobimetallic Compounds: Indium-Zinc bond cleavage}

In recent developments in the Coles' group in-house at Victoria University of Wellington $\mathrm{K}\left[\mathrm{In}\left(\mathrm{NON}^{\mathrm{Ar}}\right)\right]\left(\mathrm{NON}^{\mathrm{Ar}}=\left[\mathrm{O}\left(\mathrm{SiMe}_{2} \mathrm{~N}^{\mathrm{Ar}}\right)_{2}\right]^{2-}, \mathrm{Ar}=2,6-i \mathrm{Pr}_{2} \mathrm{C}_{6} \mathrm{H}_{3}\right)$, when comes in contact with $\mathrm{Zn}\left(\mathrm{BDI}^{\mathrm{R}}\right) \mathrm{Cl}\left(\mathrm{BDI}=\left[\mathrm{HCC}(\mathrm{Me}) \mathrm{NR}_{2}\right], \mathrm{R}=2,4,6-\mathrm{Me}_{3} \mathrm{C}_{6} \mathrm{H}_{2}(\mathrm{Mes}), \mathrm{Ar}\right)$ in the presence of $\mathrm{C}_{6} \mathrm{D}_{6}$, yields $\mathbf{I n Z n - 1}\left(\mathbf{I n Z n - 1}=\left(\mathrm{NON}^{\mathrm{Ar}}\right) \operatorname{In}-\mathrm{Zn}\left(\mathrm{BDI}^{\mathrm{R}}\right)\right)$ that further affords $\mathbf{I n Z n - 2}$ through an intermediate Int-InZn upon reacting with $\mathrm{MesN}_{3}$ as shown in Scheme 4.1 ${ }^{217}$ We first optimized the ground state geometries of these newly synthesized compounds at the PBE0-D3BJ/def2SVP level of theory. All structures were ground state minima on potential energy surface. Further, NBO and Bader analyses were performed on these optimized structures to analyze the different bonding interactions.

\subsubsection{Structural Features}

Figure 4.1 shows the ground state geometries of the three compounds given in Scheme 4.1 marked with some bond lengths including those on the reaction site. In InZn-1, In-Zn bond length is $2.56 \AA$ (experimental: $2.55 \AA$ ) which undergoes cleavage with the intoduction of mesityl azide. The intermediate (Int-InZn) was isolated and characterized, however its crystallization was not successful. It involves the attachment of both indium and zinc to the terminal nitrogen of the azide with In-N and $\mathrm{Zn}-\mathrm{N}$ bond lengths of $2.24 \AA$ and $1.93 \AA$ respectively. Additionally, the azide establishes a 4-membered 


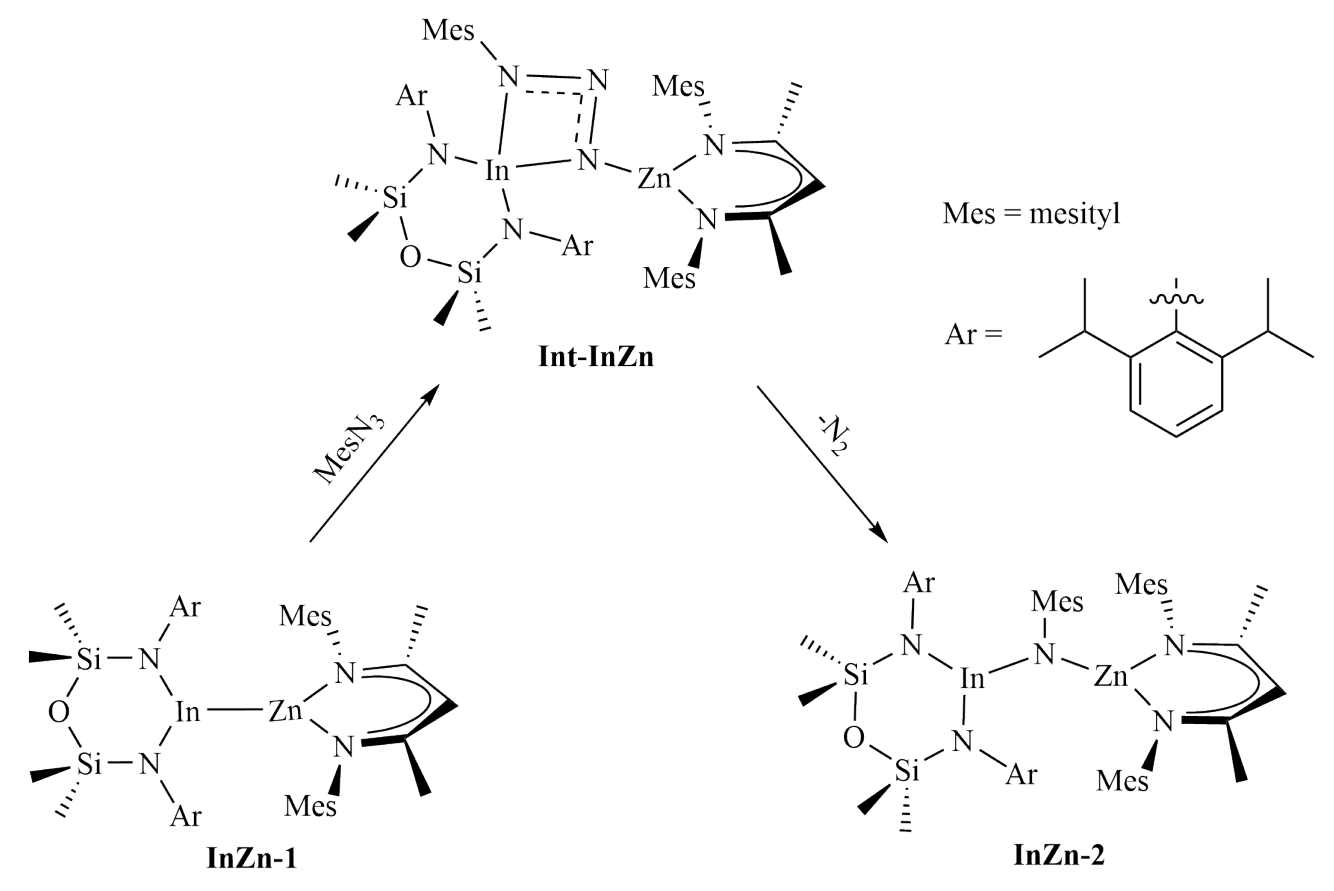

Scheme 4.1: Reaction involving the indium zinc bond in InZn-1 to add mesityl azide which results in intermediate (Int-InZn) containing 4-membered cyclic and subsequent elimination of $\mathrm{N}_{2}$ to form InZn-2. Hydrogen atoms are omitted for clarity. Reprinted from reference ${ }^{217}$ with permission (Licence ID: 1001280-1) from the publisher. Copyright (2019) Royal Society of Chemistry.

cyclic interaction with In where the second In- $\mathrm{N}_{\text {mesityl }}$ bond length is $2.28 \AA$. This latter bond is retained in the final product InZn-2 where now $\mathrm{Zn}$ is also attached to this nitrogen and the final In-N and Zn-N bond lengths are $2.04 \AA$ and $1.93 \AA$, respectively.

\subsubsection{Bonding Properties}

To gain a deeper insight in the electronic stucture and bonding properties, we carried out NBO and QTAIM analyses of compounds InZn-1 and InZn-2.

The NBO charges on In and Zn in the former are 0.86 and 1.30 respectively in InZn-1 which shows comparatively more positively charged $\mathrm{Zn}$. The Wiberg bond index that is the measure of the number of covalent bonds is 0.64 for the bond In-Zn which shows single bond character. As discussed in Chapter 2, the quantum mechanics and underlying theories such as DFT (density functional theory) offer a solution to a problem whose nature is probabilistic. Therefore the absolute values cannot be used in isola- 


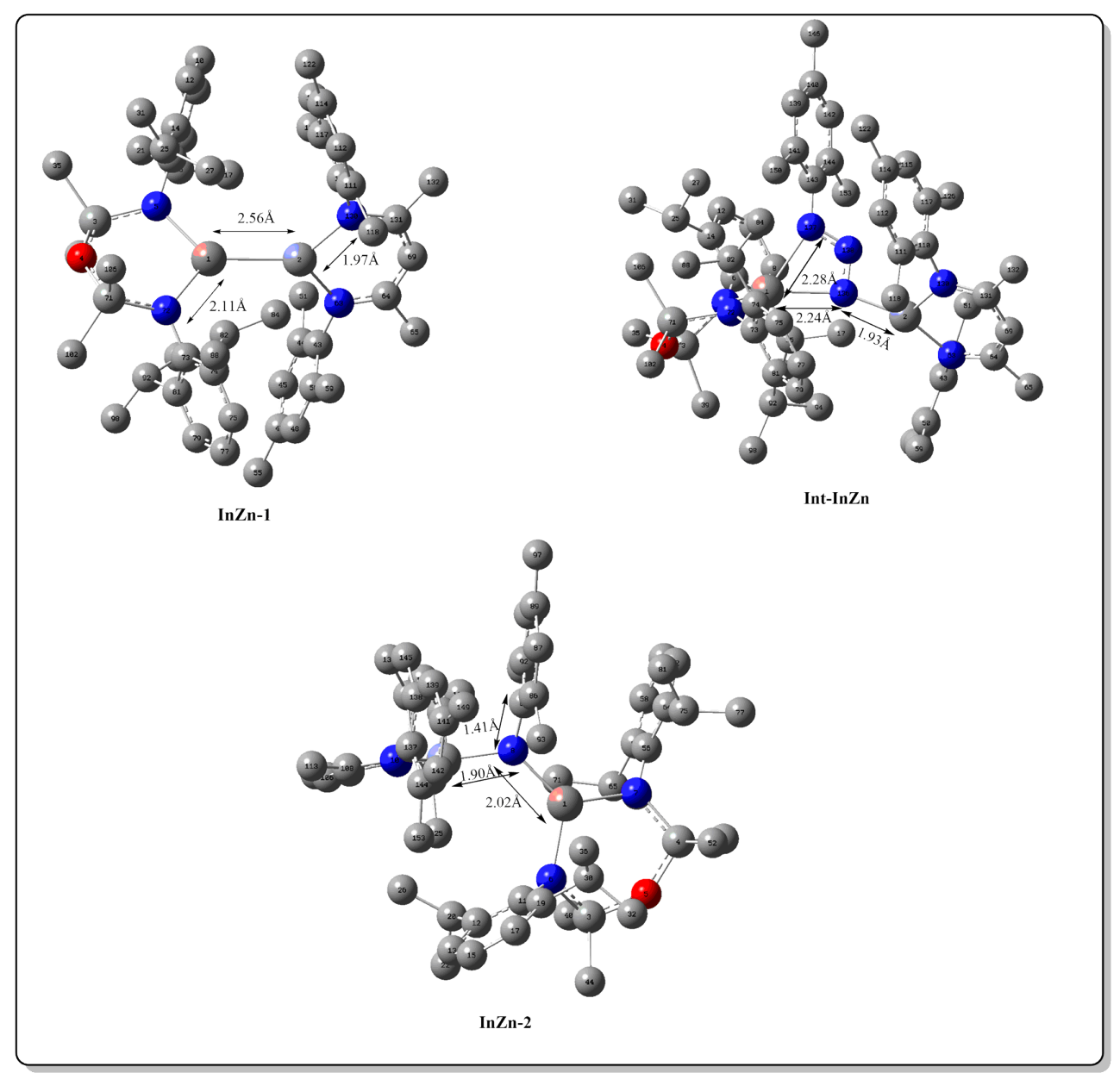

Figure 4.1: Ground state structures of the main reactant, intermediate and product in Scheme 4.1 optimized at PBE0-D3BJ/def2SVP level. Some important bond lengths including those on the reaction site have also been indicated.

tion to analyze the solution. Likewise, the WBI value of 0.64 indicates a single bond character involving one pair of electron. However, the nature of the bond whether ionic, covalent or donor-acceptor, is decided in conjunction with other indicators such as NBO and Bader's analyses. The natural bond orbital concerned with this bond shows $73.5 \%$ contribution of In while $26.5 \%$ comes from $\mathrm{Zn}$. The $s p$ hybrid orbital from In overlaps with the $\mathrm{Zn}$ orbital of mainly $p$ character (5\% contribution is from $d$ obital).

In InZn-2, there are two bonds of interest to analyze; In- $\mathrm{N}_{\text {mesityl }}$ and $\mathrm{Zn}-\mathrm{N}_{\text {mesityl }}$ since mesityl amine is inserted in In-Zn bond. The NBO charges on In, $\mathrm{Zn}$ and $\mathrm{N}_{\text {mesityl }}$ are $1.83,1.65$ and -1.45 , respectively. This shows $\mathrm{N}$ is more negatively charged as expected but now In is more positive than $\mathrm{Zn}$ unlike in InZn-1. The Wiberg bond index for In-N 
and $\mathrm{Zn}-\mathrm{N}$ bond is 0.61 and 0.23 respectively which shows a greater bond order in In-N than $\mathrm{Zn}-\mathrm{N}$.

The results for QTAIM analysis for In-Zn bond in InZn-1 and In-N and Zn-N bonds in InZn-2 are shown in Table 4.1. The nature of bond has been decided according to a set of rules given by Popelier. ${ }^{218}$ The In-Zn bond in InZn-1 is donor-acceptor where we propose, based on NBO charges, that indium is donor while zinc is acceptor as indium is more positively charged than zinc in the bond. Both the metal-nitrogen bonds in InZn-2 are proposed to be polar-covalent as the QTAIM analysis suggests. According to Popeliar ${ }^{218}$, the electron density $(\rho)$ along a bond critical point (BCP) is very low with the Laplacian of electron density $\left(\nabla^{2} \rho\right)$ having a plus $(+)$ sign. The sign with $\nabla^{2} \rho$ determines the amount of electron density: a plus sign shows the electron density is depleted along the BCP while a minus (-) sign indicates the retention of electron density accompanied by a high value of $\rho$. If a positive sign of $\nabla^{2} \rho$ is accompanied by a high electron density, then the covalent bond is comparatively a weaker shared polar interaction. Literature ${ }^{111}$ shows that an electron density of the order $10^{-2}$ represents a closed-shell interaction (ionic or donor-acceptor) while that of the order $10^{-1}$ or higher represents an open-shell interaction including covalent bond.

Table 4.1: QTAIM analysis of some important bonds in InZn-1 and InZn-2. $\rho\left(\mathrm{r}_{\mathrm{BCP}}\right)$ denotes electron density along a bond critical path $(\mathrm{BCP}), \nabla^{2} \rho\left(\mathrm{r}_{\mathrm{BCP}}\right)$ represents Laplacian of the electron density, $\mathrm{G}\left(\mathrm{r}_{\mathrm{BCP}}\right) / \rho\left(\mathrm{r}_{\mathrm{BCP}}\right)$ shows the ratio of kinetic energy to the electron density and $\mathrm{H}\left(\mathrm{r}_{\mathrm{BCP}}\right)$ shows electronic local energy density. Reprinted from reference ${ }^{217}$ with permission (Licence ID: 1001280-1) from the publisher. Copyright (2019) Royal Society of Chemistry.

\begin{tabular}{cccccc}
\hline & $\rho\left(\mathrm{r}_{\mathrm{BCP}}\right)$ & $\nabla^{2} \rho\left(\mathrm{r}_{\mathrm{BCP}}\right)$ & $\mathrm{G}\left(\mathrm{r}_{\mathrm{BCP}}\right) / \rho\left(\mathrm{r}_{\mathrm{BCP}}\right)$ & $\mathrm{H}\left(\mathrm{r}_{\mathrm{BCP}}\right)$ & Type of Bond \\
\hline $\mathrm{BCP}(\mathrm{In}-\mathrm{Zn})$ & 0.0575 & 0.0153 & 0.04443 & 0.0217 & Donor-Acceptor \\
\hline $\mathrm{BCP}(\mathrm{In}-\mathrm{N})$ & 0.1082 & 0.4533 & 1.2843 & 0.0257 & Polar-Covalent \\
\hline $\mathrm{BCP}(\mathrm{Zn}-\mathrm{N})$ & 0.1066 & 0.5345 & 1.4242 & 0.0182 & Polar-Covalent \\
\hline
\end{tabular}




\subsubsection{Conclusion}

The structural properties obtained from the crystal structure information match those of our calculated features except small deviations which usually occur due to the inclusion of crystal packing forces in experimental results that are not considered in the gas phase computational calculations. NBO and Bader's analyses were employed to explore the nature of selected bonds that will be helpful in understanding the behaviour of these complexes in future from the perspective of reactivity. The NBO charges indicate that In-Zn bond is of donor-acceptor nature in InZn-1 with the indium side behaving as donor compared to the acceptor behaviour of the zinc side. In InZn-2, the newly formed $\mathrm{Zn}-\mathrm{N}$ retains a smaller electron density than the In-N bond. 


\section{Chapter 5}

\section{Fluorescence Characteristics of}

\section{Aminobenzanthrone Dyes}

Fluorescence characteristics of 3-aminobenzanthrone derivatives are mainly attributed to intramolecular charge transfer facilitated by the extensive delocalization of the benzanthrone framework which extends its effects to the $N$-substituents. ${ }^{219}$ These electrondonating substituents attached at $\mathrm{C} 3$ of the benzanthrone framework transfer charge upon excitation via the $\pi$-conjugation system to the carbonyl group of benzanthrone ${ }^{220,221}$ as depicted in Figure 5.1 221 . This causes absorption in the region of longer wavelength. ${ }^{222}$ Moreover, this whole process is also dependent on solvent polarity; the more polar the solvent, the more enhanced intramolecular charge transfer is. ${ }^{223,224}$ The magnitude of this phenomenon is also positively affected by the stronger electron-donating capacity of the substituents. ${ }^{222}$

In the case of aminobenzanthrone dyes, the $S_{0} \rightarrow S_{1}$ excitation of a molecule results in a $\pi \rightarrow \pi^{*}$ transition $^{221}$ and subsequently, the molecules relax by emitting longer wavelength than the absorbed one. ${ }^{220}$

The current computational study is aimed at investigating the photophysics involved in the fluorescence mechanism of 3-aminobenzanthrone dyes (Figure 5.3) reported ear- 
$\operatorname{lier}^{219}$ for which the reported spectra are given in Figure 5.2. As efficient fluorophores, these dyes have also found applications as fluorescent probes for membrane studies. Trusova et al. reported their fluorescence spectra ${ }^{219}$ and both absorption and emission spectra have been again reported recently only for $\mathbf{1}_{\mathrm{AB}} \cdot{ }^{225}$

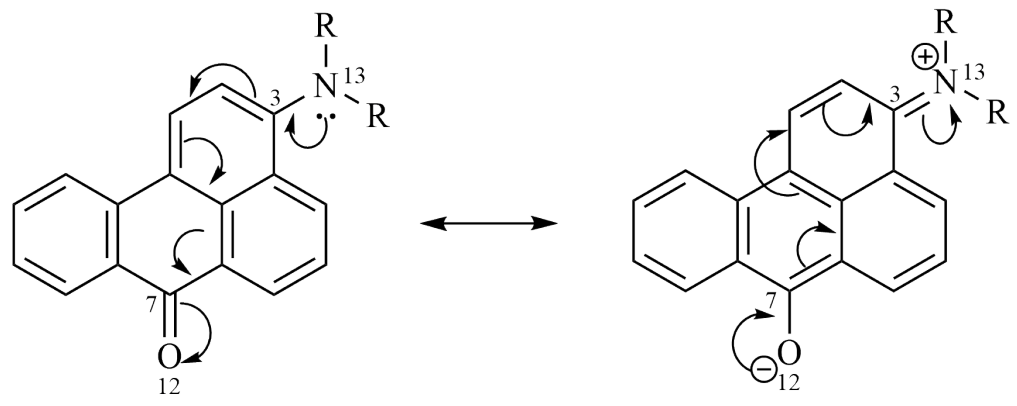

Figure 5.1: Proposed mechanism of intramolecular charge transfer in benzanthrone

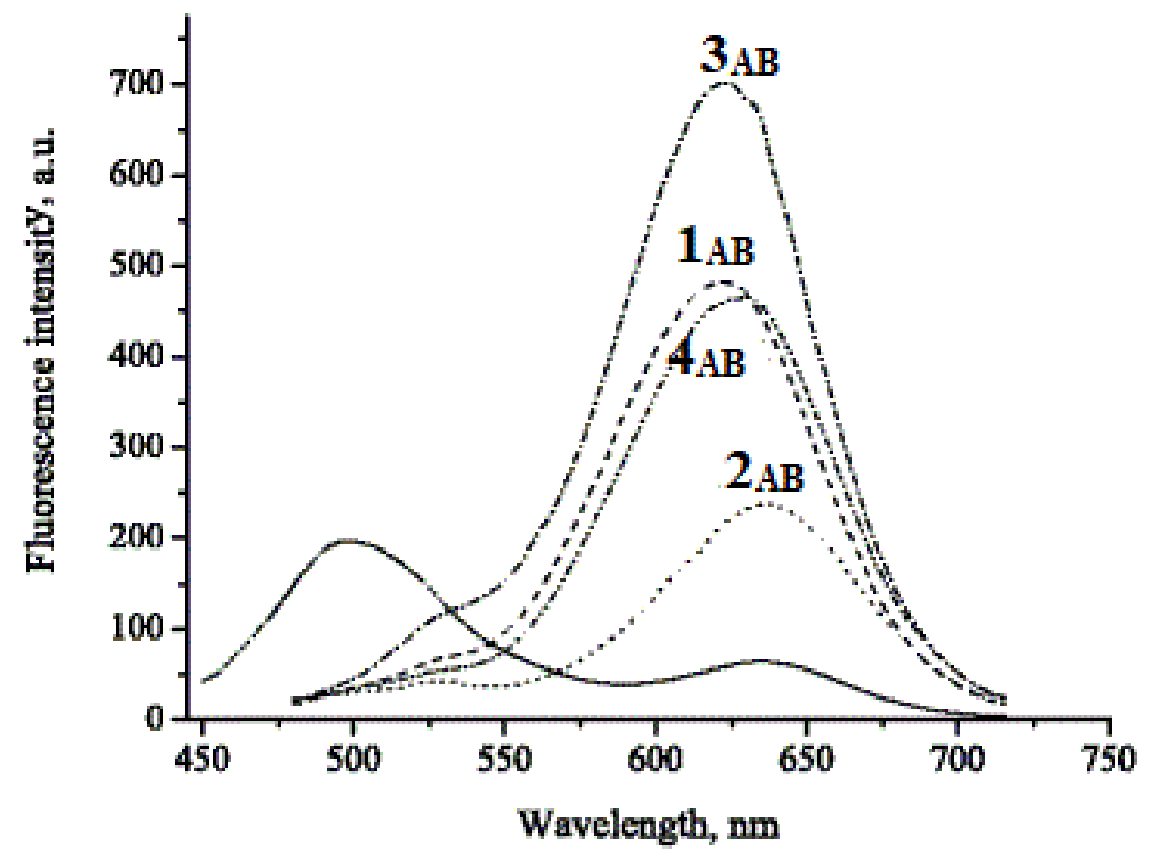

Figure 5.2: Experimentally known fluorescence spectra of the dyes under current study. ${ }^{172}$ Reprinted with permission from the copyright owner under licence number: 4694530175556. Copyright (2012) Springer Science Business Media, LLC.

To have a detailed understanding of the photophysics behind fluorescence of 3-aminobenzanthrone dyes $\left(\mathbf{1}_{\mathrm{AB}}, \mathbf{2}_{\mathrm{AB}}, \mathbf{3}_{\mathrm{AB}}\right.$ and $\left.\mathbf{4}_{\mathrm{AB}}\right)$ we first discuss the structural features of their ground state geometries both in gas phase and in ethanol solvent. This is followed by analyzing the excited state of each of these compounds. The phenomenon of absorption and emission first involves vertical excitation of a molecule followed by its 
relaxation to a new ground state. To observe the possibility of intersystem crossing, we also compared vertical excitation of each dye in its singlet state with that in its triplet state. To gain a deeper insight in the intramolecular charge transfer mechanism, we performed natural bond orbitals analysis.

\subsection{Structural Properties and Energetics of the Ground-}

\section{state Structures}

The ground state minima of all the selected dyes were optimized at PBE0-D3/def2TZVP level. All structures are local minima with no imaginary frequency. Table 5.1 presents a contrast of some of the features from these ground state geometries in gas phase and in solvated (in ethanol) phase. In addition to the comparison of the experimental bond lengths with known crystal structures of similar type such as compound (38) ${ }^{166}$, such a comparison provides a chance to see geometry differences in the two phases i.e. the gas phase and the solvent phase.

Table 5.1: Key parameters from the ground state structures both in gas phase and in solvent calculated at PBE0-D3BJ/def2TZVP level in the current study. C7=O12 is the carbonyl of benzanthrone, C3-N13 corresponds to the bond linking the amino substituents to the benzanthrone nucleus, $\phi$ reflects the dihedral angle (degrees) for the plane C2-C3-N13-C14. $\mu$ shows dipole moment (in debyes). The crystal structure data of Compound (38) ${ }^{166}$ is given for comparison as its geometry resembles that of $1_{\mathrm{AB}}$ with slight modifications on the substituent.

\begin{tabular}{|c|c|c|c|c|c|c|c|c|}
\hline & \multicolumn{4}{|c|}{ Gas Phase } & \multicolumn{4}{|c|}{ Ethanol } \\
\hline & \multicolumn{2}{|c|}{ Bond Lengths } & \multirow[t]{2}{*}{$\phi$} & \multirow[t]{2}{*}{$\mu$} & \multicolumn{2}{|c|}{ Bond Lengths } & \multirow[t]{2}{*}{$\phi$} & \multirow[t]{2}{*}{$\mu$} \\
\hline & $\mathrm{C} 7=\mathrm{O} 12$ & C3-N13 & & & $\mathrm{C} 7=\mathrm{O} 12$ & C3-N13 & & \\
\hline $\mathbf{1}_{\mathrm{AB}}$ & 1.22 & 1.39 & 16.65 & 5.16 & 1.23 & 1.37 & 14.85 & 9.6 \\
\hline $\mathbf{2}_{\mathrm{AB}}$ & 1.22 & 1.38 & -107.79 & 7.34 & 1.23 & 1.37 & -113.97 & 11.3 \\
\hline $\mathbf{3}_{\mathrm{AB}}$ & 1.22 & 1.38 & 123.67 & 7.19 & 1.23 & 1.38 & 130.27 & 11.09 \\
\hline $\mathbf{4}_{\mathrm{AB}}$ & 1.22 & 1.38 & 107.22 & 7.59 & 1.23 & 1.37 & 114.6 & 11.64 \\
\hline (38) & & & & & 1.23 & 1.37 & -3.84 & \\
\hline
\end{tabular}

As seen in the Table5.1, a computational bond length of $1.39 \AA$ for C3-N13 has been observed in $\mathbf{1}_{\mathbf{A B}}$ which is identical to the average bond length of $1.39 \AA$ reported in lit- 

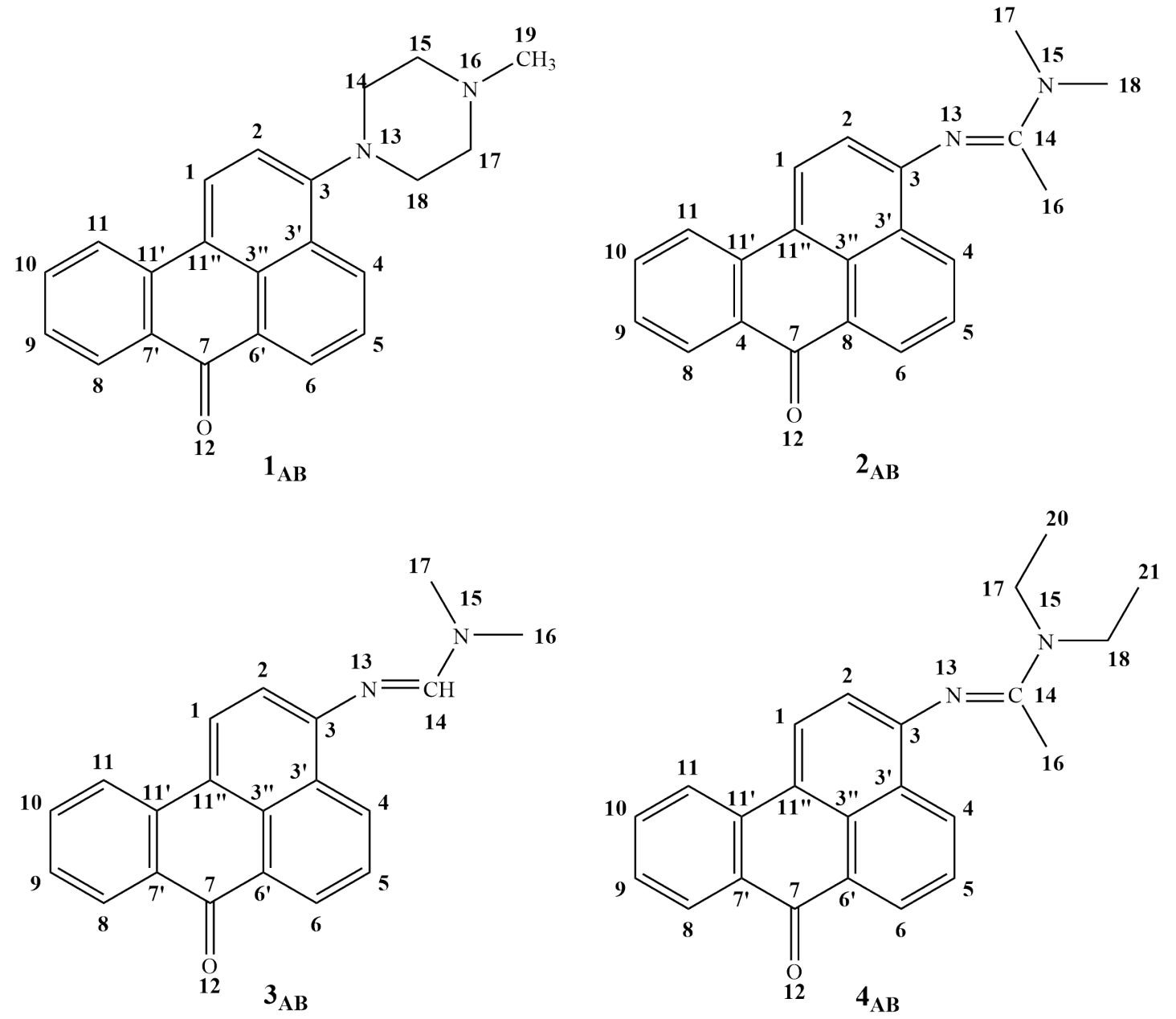

Figure 5.3: Selected 3-aminobenzanthrone dyes involved in the current study

erature $^{226}$ for $\mathrm{sp}^{3}$ hybridized nitrogen attached to an aromatic system. The bond length shrinks to $1.37 \AA$ when calculated in ethanol possibly because of enhanced intramolecular charge transfer from the basic substituent to the more electronegative ketonic carbonyl group when solvated by ethanol. This also matches C3-N13 bond length of the crystal structure of compound (38) that has a similar geometry as that of $\mathbf{1}_{\mathrm{AB}}$ except slight modification on the substituent. The C3-N13 bond length in the other three cases is the same i.e. $1.38 \AA$. This is slightly longer than the reported $1.36 \AA$ for sp ${ }^{2}$ hybridized nitrogen attached to an aromatic system. ${ }^{226}$ The deviation of the calculated bond length in the gaseous phase is a normal phenomenon because the experimentally reported values are for crystal structure (solid state) ${ }^{166}$ where the structure is condensed compared to the gas phase. 
The high polarity of the molecules is evident from the values of the dipole moment which is in the range of 5.2-7.6 D in gas phase with the highest for compound $\mathbf{4}_{\mathrm{AB}}$. The dipole moment is even higher for ethanol-solvated dyes with the values in range 9.6-11.6 D with the same trend of increasing polarity from $\mathbf{1}_{\mathbf{A B}}$ to $\mathbf{4}_{\mathrm{AB}}$ as in gas phase calculations. Hence, intramolecular charge transfer is stronger in the solvent phase.

All the molecules experience a notable change in the C2-C3-N13-C14 dihedral angle upon solvation. This means a significant difference in the structure calculated in the gas phase and in ethanol solvent. The change in dihedral angle is more pronounced in the cases where smaller substituents are attached that may easily change the orientation of attachment influenced by steric or electronic effects. The difference can also be seen in the comparison of $\mathrm{C} 2-\mathrm{C} 3-\mathrm{N} 13-\mathrm{C} 14$ dihedral angle in $\mathbf{1}_{\mathbf{A B}}$ and (38) that may be attributed to the modified substituents as well. Compound $\mathbf{1}_{\mathbf{A B}}$ with a bulkier cyclic substituent experiences a minimal change in the dihedral angle compared to the other three possibly due to steric constraints hindering the movement of the substituent in either phase. Steric constraints in this particular case may be referred to as freedom of rotation of C3-N13 affected by the attachments over N13.

\subsubsection{Stability of $\mathrm{C}=\mathrm{O}$ in Benzanthrone Framework}

The carbonyl group of the aromatic ketones has a generally unreactive $\mathrm{C}=\mathrm{O}$ bond due to extended $\pi$ conjugation. The length of this bond, therefore, is not much affected with the changing substituents on $\mathrm{C} 3$ position. It was reported earlier that repeated attempts to synthesize Schiff's bases at the carbonyl of benzanthrone had been unsuccessful. ${ }^{227}$ To our knowledge, there is no evidence of benzanthrone imino-derivatives with substituent reacting at the carbonyl site. In the current study, $\mathrm{C}=\mathrm{O}$ bond length in the four dyes is $1.22 \AA$ as observed in gas phase calculations. This implies that despite being the main receptor site in intramolecular charge transfer, the carbonyl bond is not affected by changing substituents. In solvent, the calculated bond length is $1.23 \AA$. This 
slight increase is usually due to $\mathrm{C}=\mathrm{O}$.... H-O hydrogen bonding between benzanthrone and ethanol which is, to a certain extent, simulated by the SMD model. Although the hydrogen bonding interactions are not explicitly calculated by it, this solvent model is mainly an electrostatic model that makes cavities around the solute molecules to estimate electrostatic interactions between the solute and the solvent implicitly.

\subsubsection{NBO Analysis}

To obtain an extended picture of the Lewis structures of the molecules, NBO analysis was carried out. We used the ground state structures to run the calculations. All the calculations were performed in NBO 6.0 as implemented in Gaussian 09 using PBE0D3 functional and def2TZVP basis set. In addition to the electronic information about the atoms, this analysis is useful in predicting the increase in polarization of ethanolsolvated dyes due to the polar solvent.

Table 5.2 shows the NBO charges on selected atoms from all the molecules involved in the current study. It can be observed that the NBO charges of the gas phase and ethanol phase ground state dyes do not change significantly. However, the small changes in the numerical values supports the concept of enhanced polarity in a polar solvent such as ethanol compared to the gas phase ground states of these dyes.

Figure 5.4 shows the charge transfer in the selected benzanthrone dyes calculated through the second order peturbation theory as implemented in $N B O$ 6.0. The numbers in the figure show the energy of each transfer in $\mathrm{kcal} / \mathrm{mol}$. The higher the energy the stronger the charge transfer interaction will be. 
Table 5.2: NBO charges of some significant atoms

\begin{tabular}{c|cccc|cccc}
\hline & \multicolumn{4}{|c}{ Gas Phase } & \multicolumn{4}{c}{ Ethanol } \\
\hline & $\mathbf{1}_{\mathbf{A B}}$ & $\mathbf{2}_{\mathrm{AB}}$ & $\mathbf{3}_{\mathrm{AB}}$ & $\mathbf{4}_{\mathbf{A B}}$ & $\mathbf{1}_{\mathbf{A B}}$ & $\mathbf{2}_{\mathrm{AB}}$ & $\mathbf{3}_{\mathrm{AB}}$ & $\mathbf{4}_{\mathrm{AB}}$ \\
\hline C3 & 0.21 & 0.19 & 0.18 & 0.19 & 0.22 & 0.19 & 0.19 & 0.20 \\
C7 & 0.51 & 0.51 & 0.51 & 0.51 & 0.50 & 0.50 & 0.50 & 0.50 \\
O12 & -0.55 & -0.55 & -0.55 & -0.55 & -0.56 & -0.56 & -0.56 & -0.56 \\
N13 & -0.40 & -0.52 & -0.52 & -0.53 & -0.39 & -0.52 & -0.53 & -0.53 \\
N15 & & -0.38 & -0.38 & -0.39 & & -0.38 & -0.37 & -0.38 \\
N16 & -0.42 & & & & -0.41 & & & \\
C14 & & 0.48 & 0.30 & 0.48 & & 0.47 & 0.29 & 0.48 \\
C16 & & -0.71 & -0.44 & -0.71 & & -0.71 & -0.43 & -0.71 \\
C17 & & -0.43 & -0.42 & -0.22 & & -0.43 & -0.41 & -0.22 \\
C18 & & -0.42 & & -0.63 & & -0.42 & & -0.63 \\
C19 & -0.43 & & & -0.23 & -0.43 & & & -0.23 \\
C20 & & & & -0.63 & & & & -0.63 \\
\hline
\end{tabular}

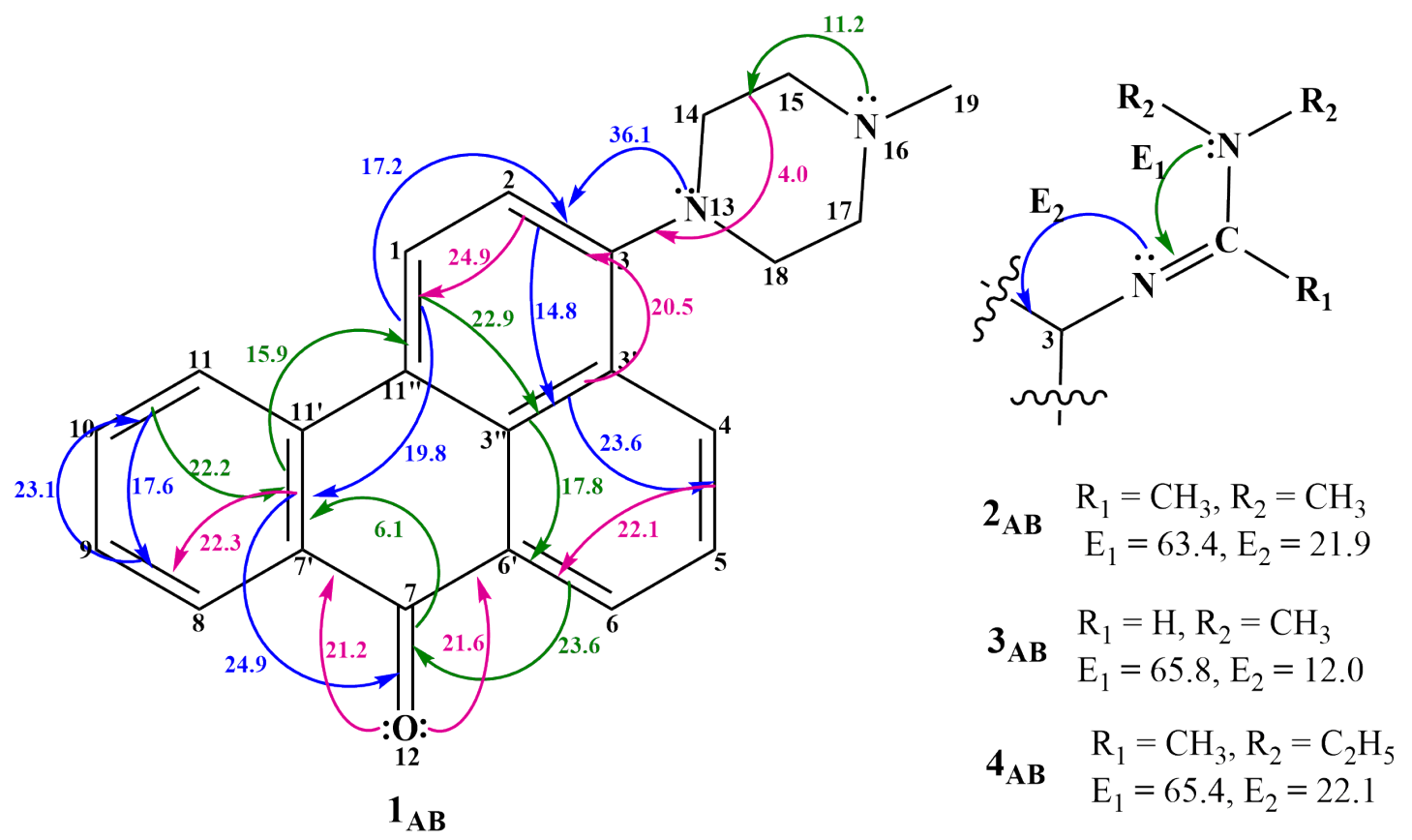

Figure 5.4: Elaboration of charge transfer in ethanol phase from occupied to unoccupied orbitals as observed in selected benzanthrone dyes through second order perturbation theory (employed in NBO analysis). Since the mechanism is same (with small difference in the intensity of each transfer) in benzanthrone framework of all the molecules, only the substituents are given for $\mathbf{2}_{\mathrm{AB}}, \mathbf{3}_{\mathrm{AB}}$ and $\mathbf{4}_{\mathrm{AB}}$. Varying colours are intended for clarity. All energies are in $\mathrm{kcal} / \mathrm{mol}$.

\subsubsection{FMO Analysis}

A frontier molecular orbital (FMO) diagram can provide an insight into the reactivity of a molecule. However, in our case it also provides useful information about the HOMO- 
LUMO band gap by calculating the change in SCF energy of both the molecular orbitals as an electron moves from one to the other and vice-versa. The HOMO and LUMO of all the benzanthrone dyes in the gas phase ground states are shown in Figure 5.5.

The band gaps calculated for the gas phase and solvent phase ground states are given in Table 5.3 It is evident that the band gap decreases with the increase in polarity that makes an electronic excitation easier in these dyes. The gas phase ground state band gap in case of $\mathbf{1}_{\mathbf{A B}}$ is $3.39 \mathrm{eV}$ while in ethanol it reduces to $3.05 \mathrm{eV}$. The trends for all the dyes are similar to $\mathbf{1}_{\mathbf{A B}}$, however, there may be differences in the energy in the same phase of different dyes. $\Delta \mathrm{E}_{\mathrm{SCF}}$ for $\mathbf{2}_{\mathrm{AB}}$ is $3.50 \mathrm{eV}$ and $3.37 \mathrm{eV}$ in gas phase ground state and solvated ground state respectively. For dyes $\mathbf{3}_{\mathrm{AB}}$ and $\mathbf{4}_{\mathrm{AB}}$ in the similar order the band gaps are $3.48 \mathrm{eV}, 3.23 \mathrm{eV}$ and $3.47 \mathrm{eV}, 3.22 \mathrm{eV}$ respectively.

Table 5.3: Band gap calculated as a change in SCF energy between the gas and ethanol phase optimized frontier molecular orbitals. All energies in eV.

\begin{tabular}{l|c|c}
\hline & Ground-state Gas Phase & Ground-state Ethanol phase \\
\hline $\mathbf{1}_{\mathrm{AB}}$ & 3.39 & 3.05 \\
$\mathbf{2}_{\mathrm{AB}}$ & 3.50 & 3.37 \\
$\mathbf{3}_{\mathrm{AB}}$ & 3.48 & 3.23 \\
$\mathbf{4}_{\mathrm{AB}}$ & 3.47 & 3.22 \\
\hline
\end{tabular}

\subsection{Excited States}

To understand the photophysics involved in the flourescence behaviour of our selected 3-aminobenzanthrone dyes, we calculated the energies of their excited states in both gaseous and solvated phases. We used time-dependent DFT (TDDFT) and all the calculations were at PBE0-D3/def2TZVP level of theory. The ground state minima optimized at the same level of DFT were used as the geometries to calculate vertical excitation energies. We first calculated the vertical excitation energies for singlet states (for a discussion of the triplet states see below). The state of interest that we employed for all the molecules was first excited state as it is evident from the reported spectra of the molecules that only this excited state is involved in giving absorption peaks. We fur- 

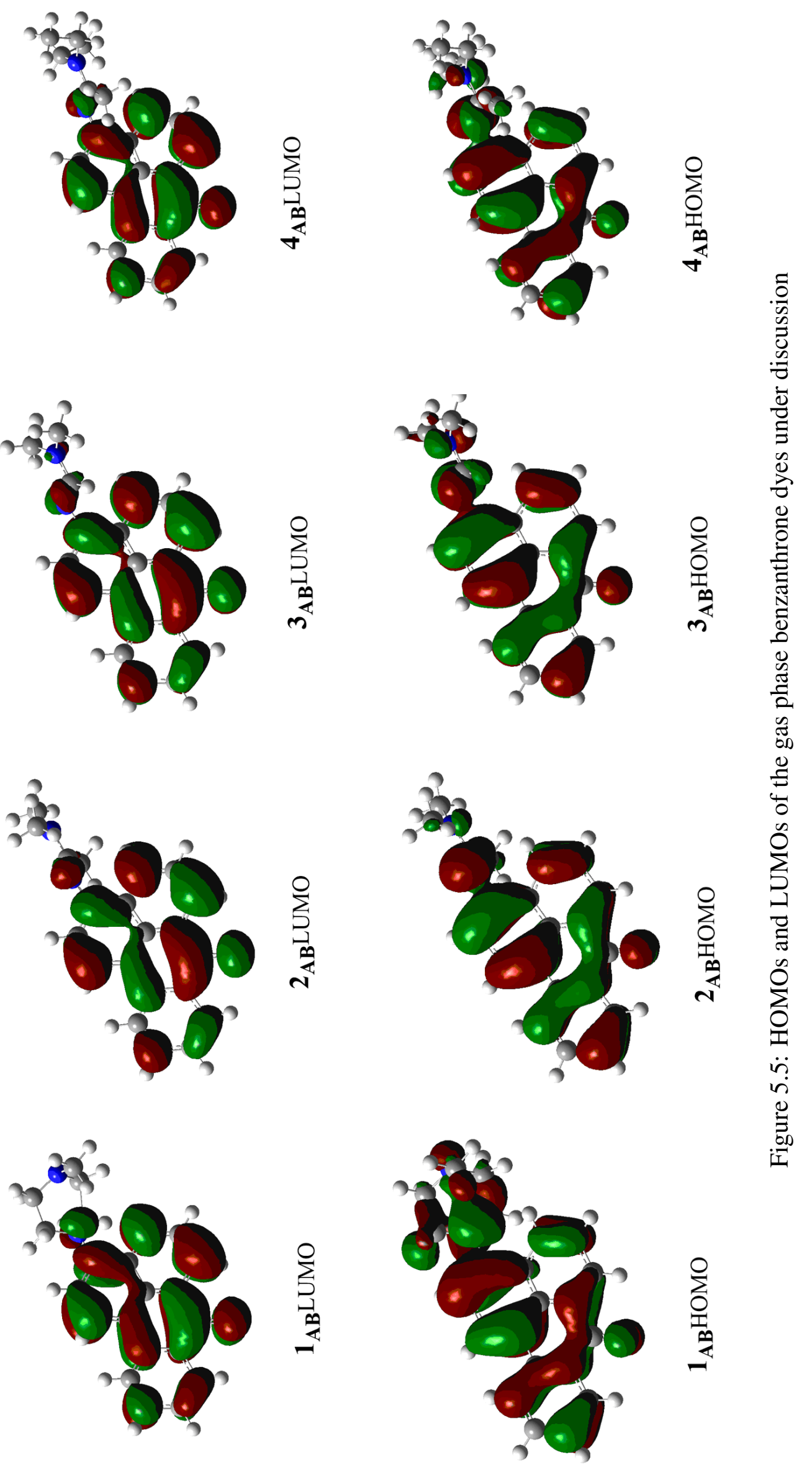
ther optimized the structure of the excited state in each case for both free and solvated molecules. To explore the possibilities of intersystem crossing during fluorescence, we calculated the triplet state vertical excitations for all the molecules in ethanol.

\subsubsection{Vertical Excitations}

Table 5.4 shows the energy change (absorbed) when the ground state of each molecule reaches the first excited state, the corresponding dipole moment and oscillator strength for gaseous phase calculations as well as for calculations in ethanol-solvated phase. We calculated this only for the first excited state in each case since the oscillator strength is highest for this state and the available experimental data shows no involvement of higher excited states in the fluorescence process. The experimentally determined spectra have a single absorption maximum for these molecules ${ }^{225}$ which corresponds to $\Delta \mathrm{E}_{\mathrm{abs}}$ calculated for the excited state with highest oscillator strength. The oscillator strength does not have any unit and it measures the strength of electronic transitions. ${ }^{228}$ The higher its value, the more intense the transition.

Table 5.4: Results of Vertical excitations highlighting absorbed energy $\left(\Delta \mathrm{E}_{\mathrm{abs}}\right)$ in $\mathrm{eV}$, oscillator strength $(f)$ and dipole moment $(\mu)$ in debye for the first excited state.

\begin{tabular}{cccc|ccc}
\hline & \multicolumn{3}{c}{ Gas Phase } & \multicolumn{3}{c}{ Ethanol } \\
\hline & $\Delta \mathrm{E}_{\mathrm{abs}}$ & $f$ & $\mu$ & $\Delta \mathrm{E}_{\mathrm{abs}}$ & $f$ & $\mu$ \\
\hline $\mathbf{1}_{\mathrm{AB}}$ & 2.77 & 0.25 & 10.1 & 2.39 & 0.46 & 17.7 \\
$\mathbf{2}_{\mathrm{AB}}$ & 2.88 & 0.26 & 10.5 & 2.59 & 0.51 & 16.5 \\
$\mathbf{3}_{\mathrm{AB}}$ & 2.88 & 0.27 & 10.6 & 2.55 & 0.54 & 17.2 \\
$\mathbf{4}_{\mathrm{AB}}$ & 2.85 & 0.27 & 11.0 & 2.55 & 0.51 & 17.6 \\
\hline
\end{tabular}

The calculated gas phase values of the oscillator strength do not vary much for all the dyes as evident from the table. However, its value is consistently higher for all the vertical excitations in ethanol compared to that in gas phase. The highest oscillator strength is recorded for solvated $\mathbf{3}_{\mathbf{A B}}$ that is 0.54 . The same value of $f(0.51)$ for $\mathbf{2}_{\mathbf{A B}}$ and $\mathbf{4}_{\mathrm{AB}}$ predicts the absorption peak of similar intensity for these two but somewhat more intense than the absorption peak of $\mathbf{1}_{\mathbf{A B}}$ as predicted by its oscillator strength $(0.46)$. 
The gas phase calculations show that the change in energy absorbed is only affected to a small extent by a change in substituents on the benzanthrone framework. The molecules $\mathbf{2}_{\mathrm{AB}}$ and $\mathbf{3}_{\mathrm{AB}}$ even show identical value of $\Delta \mathrm{E}_{\mathrm{abs}}$ which is $2.88 \mathrm{eV}$. Energy absorbed by $\mathbf{4}_{\mathrm{AB}}$ is on second with a value of $2.85 \mathrm{eV}$ and the lowest absorption energy gap (2.77 $\mathrm{eV}$ ) has been predicted for $\mathbf{1}_{\mathbf{A B}}$ in gas phase. The solvent phase absorption is markedly different from that of gas phase molecules. Moreover, absorption is significantly different in ethanol from dye to dye. The solvent shifts the absorption towards the region of longer wavelength. The biggest red shift is hereby reported for $\mathbf{1}_{\mathbf{A B}}$ with the absorption of $2.39 \mathrm{eV}(518.30 \mathrm{~nm})$. There is approximately $12 \%$ error in this value from the reported $455.4 \mathrm{~nm}$ which is in range of the already reported errors of $13 \%{ }^{199}$ and $23 \%{ }^{229}$ in such calculations. There is no experimental absorption data for the other dyes to the best of our search.

While comparing the gas phase calculations with those of solvated phase, we note that the dipole moment is markedly enhanced for all the dyes in the latter. It increases by about 7.6 $\mathrm{D}$ in $\mathbf{1}_{\mathbf{A B}}$ which is the highest recorded difference in all the molecules. Ethanol-solvated $\mathbf{2}_{\mathrm{AB}}$ experiences a rise of $6.0 \mathrm{D}$ from gas phase, while, changing from free phase to solvation in both $\mathbf{3}_{\mathbf{A B}}$ and $\mathbf{4}_{\mathbf{A B}}$ results an increase of $6.6 \mathrm{D}$ each. The comparison of ground state calculations with that of excited state shows even bigger differences. Excitation results in the increase of polarity. It almost doubles in $\mathbf{1}_{\mathbf{A B}}$ where the piperazino substituent has all the atoms $\mathrm{sp}^{2}$ hybridized which is more vulnerable to such changes from other dyes. The latter have an additional double bond in the substitutents which, although expected to undergo a $\pi \rightarrow \pi^{*}$ transition, enables them to resist the change in dipole moments across the N13=C14 bond (Table 5.5).

Table 5.5: Dipole moment of ethanol-solvated dyes in their ground state $\mu_{\mathrm{GS}}$ and in excited state $\mu_{\mathrm{ES}}$

\begin{tabular}{c|c|c}
\hline & $\mu_{\mathrm{GS}}$ & $\mu_{\mathrm{ES}}$ \\
\hline $\mathbf{1}_{\mathbf{A B}}$ & 9.6 & 17.7 \\
$\mathbf{2}_{\mathbf{A B}}$ & 11.3 & 16.5 \\
$\mathbf{3}_{\mathbf{A B}}$ & 11.09 & 17.2 \\
$\mathbf{4}_{\mathbf{A B}}$ & 11.64 & 17.6 \\
\hline
\end{tabular}




\subsubsection{NTO Analysis}

As stated earlier, fluorescence in benzanthrone derivatives is the result of intramolecular charge transfer. To get a deeper insight on this phenomenon, we performed natural transition orbitals (NTO) analysis of all the dyes in gas phase and ethanol phase when they undergo vertical excitation. A difference of charge density in the highest occupied transition orbital (HOTO) and the lowest unoccupied transition orbital (LUTO) is shown in Fig 5.6. The HOTO is indicated by a violet colour while a light blue colour denotes the LUTO. Differences can be observed by comparing the diagram of these NTOs for vertical excitations in gas phase to that in ethanol solvent.

It can be clearly seen that the amount of charge transferring from the HOTO present on N16 markedly increases in ethanol phase compared to that in gas phase which is indicative of enhanced charge transfer in the former. There are many other differences

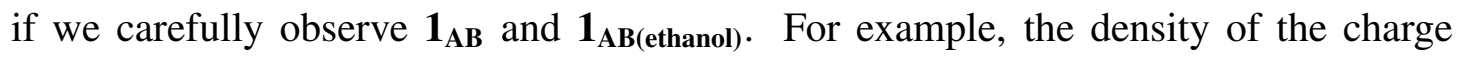
transferring from the HOTO present on $\mathrm{C} 9$ and $\mathrm{C} 11$ " decreases in the solvated dye. Moreover, there is enhanced transfer of charge from the HOTO present on bonds C15C16 and C17-C18. The charge density in the LUTO mainly resides on or near to the carbonyl group. It can be inferred from this discussion that the charge transfer from the HOTO is scattered over the whole molecule in the gas phase. On the contrary, in the solvent phase the charge transfer from the HOTO is more localized at the $\mathrm{N}$ substituent. Similar differences can be observed in the charge density of $\mathbf{2}_{\mathbf{A B}}, \mathbf{3}_{\mathbf{A B}}$ and $\mathbf{4}_{\mathrm{AB}}$ by comparing their NTOs present in free and solvated structures. In all cases, the donation of charge density from the HOTO of N15 is more pronounced in solvated phase. 


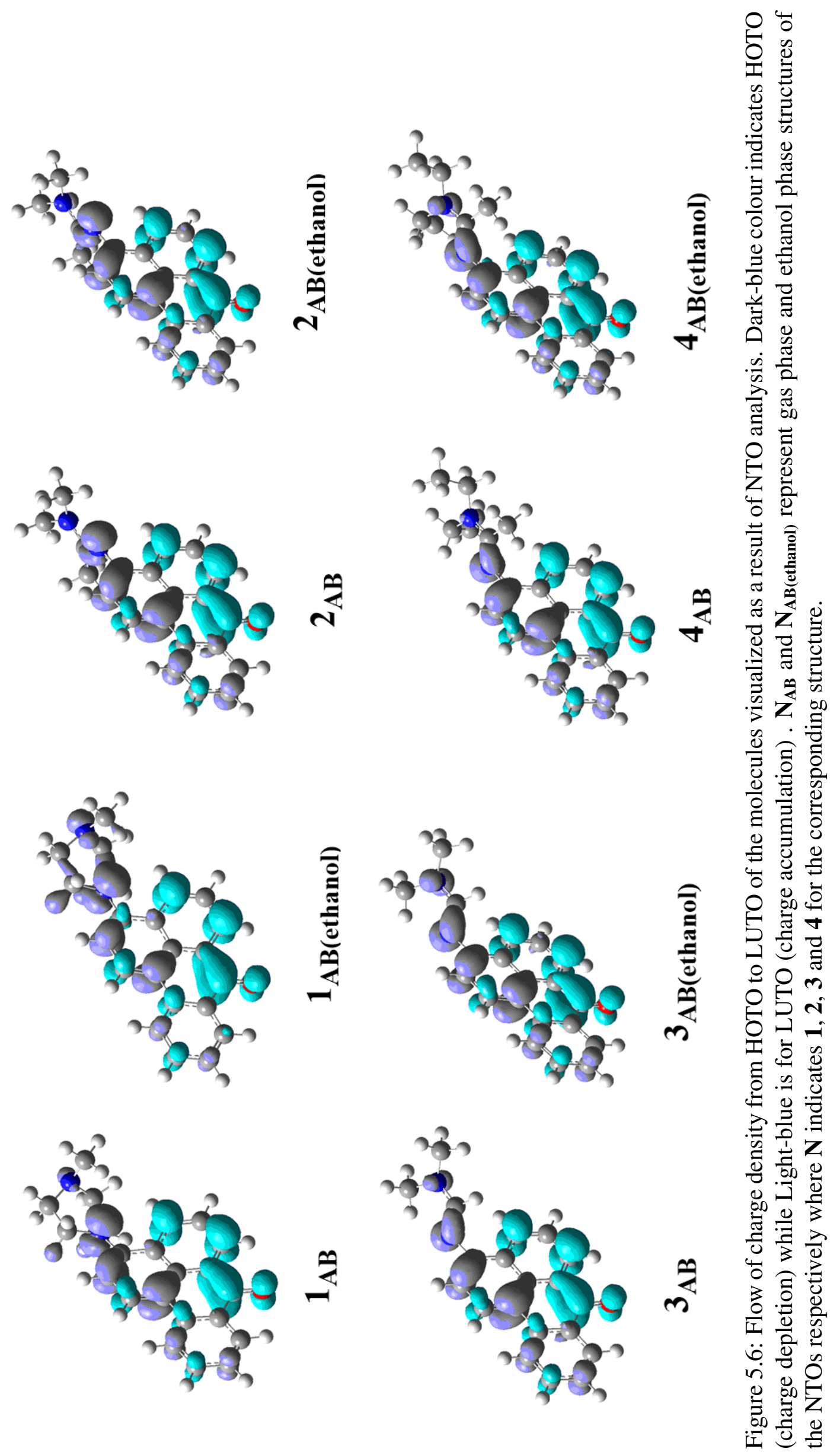




\subsubsection{Excited State Optimization}

The data obtained from optimizing the geometries of the first excited state of all the molecules in both gas phase and ethanol phase at PBE0-D3/def2TZVP level of TDDFT is listed in Table 5.6. While moving from gas phase to solvated, there is a red shift in the emitted energy. This red-shift is approximately $78 \mathrm{~nm}(0.36 \mathrm{eV})$ for $\mathbf{1}_{\mathbf{A B}}, \mathbf{2}_{\mathbf{A B}}$ and $\mathbf{4}_{\mathbf{A B}}$ while it is about $72 \mathrm{~nm}(0.35 \mathrm{eV})$ for $\mathbf{3}_{\mathrm{AB}}$. These values are indicative of enhanced charge transfer in the solvated phase where the polarity of ethanol is mainly responsible for this difference. All the calculated emission maxima can be correlated well with the experimental results ${ }^{219}$ where the fluorescence spectra are in the range of 625-635 nm. The calculated $\Delta \mathrm{E}_{\mathrm{ems}}$ is $610.23 \mathrm{~nm}(2.03 \mathrm{eV})$ for $\mathbf{1}_{\mathrm{AB}}, 606.71 \mathrm{~nm}(2.04 \mathrm{eV})$ for $\mathbf{2}_{\mathrm{AB}}$ and $\mathbf{4}_{\mathbf{A B}}$ and $590.95 \mathrm{~nm}(2.10 \mathrm{eV})$ for $\mathbf{3}_{\mathbf{A B}}$. We report a 5-6\% error while comparing these calculations with the experimental results. This deviation is quite low compared to the already reported $23 \%$ error $^{229}$ typical for such types of electronic transitions.

Table 5.6: Results of excited state optimization highlighting the energy emitted $\left(\Delta \mathrm{E}_{\text {ems }}\right)$ in $\mathrm{eV}$, oscillator strength $(f)$ and dipole moment $(\mu)$ in debye for the first excited state. $\phi$ reflects the dihedral angle (degrees) for the plane C2-C3-N13-C14.

\begin{tabular}{l|cccc|cccc}
\hline & \multicolumn{4}{|c}{ Gas Phase } & \multicolumn{4}{c}{ Ethanol } \\
\hline & $\Delta \mathrm{E}_{\text {ems }}$ & $\phi$ & $f$ & $\mu$ & $\Delta \mathrm{E}_{\text {ems }}$ & $\phi$ & $f$ & $\mu$ \\
\hline $\mathbf{1}_{\mathbf{A B}}$ & 2.39 & 23.90 & 0.21 & 6.19 & 2.03 & 26.22 & 0.47 & 11.12 \\
$\mathbf{2}_{\mathbf{A B}}$ & 2.40 & -133.01 & 0.24 & 8.58 & 2.04 & -139.99 & 0.52 & 14.62 \\
$\mathbf{3}_{\mathrm{AB}}$ & 2.45 & 141.03 & 0.25 & 8.33 & 2.10 & 146.10 & 0.57 & 14.01 \\
$\mathbf{4}_{\mathbf{A B}}$ & 2.36 & 133.51 & 0.25 & 8.89 & 2.04 & 140.94 & 0.53 & 14.90 \\
\hline
\end{tabular}

We observed significant difference in oscillator strength between the gas phase and ethanol phase optimization of the excited state. In the gas phase, it is nearly identical in all the cases, however, based on the numerical values, it can be given in the decreasing order as $\mathbf{4}_{\mathrm{AB}}=\mathbf{3}_{\mathrm{AB}}>\mathbf{2}_{\mathrm{AB}}>\mathbf{1}_{\mathrm{AB}}$. In ethanol, however, it is highest for $\mathbf{3}_{\mathrm{AB}}$ which corresponds to its most intense peak in the fluorescence spectra of these compounds (Figure 5.2. ${ }^{219}$ The change in C2-C3-N13-C14 dihedral angle of the excited state minimum is also significant upon solvation. This changes by $7.43^{\circ}$ in $\mathbf{4}_{\mathrm{AB}}$ and $6.98^{\circ}$ in $\mathbf{2}_{\mathrm{AB}}$ which suggests that the structure changes upon solvation. In this way the geometry of $\mathbf{3}_{\mathrm{AB}}$ also undergoes significant change as the dihedral angle under discussion increases by $5.07^{\circ}$. 
However, $\mathbf{1}_{\mathbf{A B}}$ is least affected where this is just $2.32^{\circ}$ changed. These changes can be explained on the basis of the the rotation of the substituents. The cyclic substituent in $\mathbf{1}_{\mathrm{AB}}$ does not allow the solvent to induce planarity to much extent so the change in dihedral angle is small. The structure of the other three molecules undergo a greater change toward planarity which is attributed to the enhanced ability of the substituents to rotate across the benzanthrone framework.

An increase in polarity is further supported by the difference in dipole moment between the two phases. The dipole moment is markedly enhanced for all the dyes when we compare the gas phase excited state optimization with that of solvated phase. Dye $\mathbf{2}_{\mathbf{A B}}$ experiences the biggest increase of $6.04 \mathrm{D}$ while $\mathbf{4}_{\mathbf{A B}}$ take the second position with an increase of 6.01 D. Ethanolated $\mathbf{3}_{\mathrm{AB}}$ is $5.68 \mathrm{D}$ more polar than its gas phase counterpart and the least affected by solvent polarity is $\mathbf{1}_{\mathrm{AB}}$ with $4.93 \mathrm{D}$ increase in dipole moment. Changes in the dipole moment can also be observed while transitioning from vertical excitations to the excited state minima (Table5.5). Since the vertically excited molecule is at a higher vibrational level with distorted electronic structure, its relaxation to the excited state minimum with a reduced electronic distortion causes the dipole moment to decrease.

Table 5.5 also shows the Stokes' shift for the dyes under discussion. It is calculated as a difference of $\Delta \mathrm{E}_{\mathrm{abs}}$ and $\Delta \mathrm{E}_{\text {ems. }}$. We can see that highest Stokes' shifted emission is for $\mathbf{2}_{\mathrm{AB}}$ while it is the lowest for $\mathbf{1}_{\mathrm{AB}}$. A careful observation shows that Stokes' shift has an inverse relationship with the dipole moment. The more the polarity decreases as a result of the relaxation of vertically excited molecule to the excited state minimum, the smaller the Stokes' shift. This means that the dyes which retain higher polarity induced as a result of vertical excitation even in their excited state minima fluoresce with more bathochromic shift compared to the case where the dipole moment decreases to a great extent $\left(\mathbf{1}_{\mathbf{A B}}\right)$. 
Table 5.7: Dipole moment of ethanol-solvated dyes on their absorption maxima $\mu_{\text {abs }}$ and after relaxation of the excited states to their minima $\mu_{\mathrm{ems}}$. Stokes shift (S.S.) can be calculated as the difference of the absorbed energy as a result of vertical excitation and emission energy resulting from relaxation of the excited state minimum back to the ground state

\begin{tabular}{l|c|c|c|c|c}
\hline & $\Delta \mathrm{E}_{\text {abs }}$ & $\mu_{\text {abs }}$ & $\Delta \mathrm{E}_{\text {ems }}$ & $\mu_{\text {ems }}$ & S.S. \\
\hline $\mathbf{1}_{\mathbf{A B}}$ & 2.39 & 17.7 & 2.03 & 11.1 & 0.36 \\
$\mathbf{2}_{\mathbf{A B}}$ & 2.58 & 16.5 & 2.04 & 14.6 & 0.54 \\
$\mathbf{3}_{\mathrm{AB}}$ & 2.55 & 17.2 & 2.10 & 14.0 & 0.45 \\
$\mathbf{4}_{\mathbf{A B}}$ & 2.55 & 17.6 & 2.04 & 14.9 & 0.51 \\
\hline
\end{tabular}

\subsubsection{Comparison of Solvated Ground State and Excited State Struc- tures}

It can be inferred from the discussion in the previous section that when the excited state of a molecule dissolved in a polar solvent relaxes to its minimum, its dipole moments are realigned with that of solvent and significant changes can be observed in the geometry. Table 5.8 presents a comparison between the ethanol-solvated ground state and excited state geometries of all the molecules under study. As expected, under the effects of high energy as a result of absorption in addition to the dipole interactions and hydrogen bonding with solvent molecules, $\mathrm{C}=\mathrm{O}$ bond lengths significantly increase from 1.23 $\AA$ in all the ground state molecules to $1.26 \AA$ in their corresponding excited states. Moreover, enhanced electron donation from the substituents at C3 decrease the C3-N13 bond length. The shortest distance is $1.34 \AA$ in $\mathbf{2}_{\mathrm{AB}}$ and $\mathbf{4}_{\mathrm{AB}}$ which is correlated with the increased number of electron-donating alkyl groups present compared to $\mathbf{3}_{\mathrm{AB}}$ where C3-N13 remains 1.35 ̊. This bond is least perturbed in $\mathbf{1}_{\mathbf{A B}}$ with bond length of 1.36 $\AA$ possibly due to the steric resistance posed by the cyclic substituent.

Marked changes in structure can be further observed from the altered values of dihedral angles for the plane C2-C3-N13-C14 upon excitation. We report a shift towards planarity in all the excited state structures. The large increase can be witnessed in $\mathbf{2}_{\mathbf{A B}}$ $\left(26.02^{\circ}\right)$ and $\mathbf{4}_{\mathrm{AB}}\left(26.34^{\circ}\right)$ where $\mathrm{C} 14$ doubly bonded to N13 is further bonded to bulkier electron donating moieties which cause C3-N13 to shorten and ultimately contribute to 
bringing the geometry toward planarity. The change of $11.37^{\circ}$ in the dihedral angle for the plane under discussion in $\mathbf{1}_{\mathbf{A B}}$ can be explained on the basis of steric factors due to the cyclic piperazino substituent which resists to a change in structure upon excitation that occurs in others through the rotation of the substituent. The geometry of $\mathbf{3}_{\mathrm{AB}}$ also undergoes change in the excited state, evident from the $15.83^{\circ}$ increase in $\mathrm{C} 2-\mathrm{C} 3-\mathrm{N} 13-$ C14 the dihedral angle. Dipole moments are also different in both states for all the molecules. These results are consistent with the already known concept of increasing polarity upon excitation of a ground state geometry to an excited state. ${ }^{229,230}$

Table 5.8: Comparison of key parameters from the solvated phase ground state and excited state structures of the dyes under discussiom. $\mathrm{C} 7=\mathrm{O} 12$ is the carbonyl of benzanthrone, C3-N13 corresponds to the bond linking the amino substituents to the benzanthrone nucleus, $\phi$ reflects the dihedral angle (degrees) for the plane C2-C3-N13-C14. $\mu$ shows dipole moment (in debyes)

\begin{tabular}{|c|c|c|c|c|c|c|c|c|}
\hline & \multicolumn{4}{|c|}{ Ground state } & \multicolumn{4}{|c|}{ Excited State } \\
\hline & \multicolumn{2}{|c|}{ Bond Lengths } & \multirow[t]{2}{*}{$\phi$} & \multirow[t]{2}{*}{$\mu$} & \multicolumn{2}{|c|}{ Bond Lengths } & \multirow[t]{2}{*}{$\phi$} & \multirow[t]{2}{*}{$\mu$} \\
\hline & $\mathrm{C} 7=\mathrm{O} 12$ & C3-N13 & & & $\mathrm{C} 7=\mathrm{O} 12$ & C3-N13 & & \\
\hline $\mathbf{1}_{\mathrm{AB}}$ & 1.23 & 1.37 & 14.85 & 9.6 & 1.26 & 1.36 & 26.22 & 11.12 \\
\hline $2_{\mathrm{AB}}$ & 1.23 & 1.37 & -113.97 & 11.3 & 1.26 & 1.34 & -139.99 & 14.62 \\
\hline $\mathbf{3}_{\mathrm{AB}}$ & 1.23 & 1.38 & 130.27 & 11.09 & 1.26 & 1.35 & 146.10 & 14.01 \\
\hline $\mathbf{4}_{\mathrm{AB}}$ & 1.23 & 1.37 & 114.6 & 11.64 & 1.26 & 1.34 & 140.94 & 14.90 \\
\hline
\end{tabular}

\subsubsection{Triplet State Vertical Excitations}

The singlet state of a molecule is characterized by the paired spins of electrons, even after excitation. If, however, any two electrons at the same energy level have unpaired spin as a result of excitation or de-excitation, the molecule is said to be in triplet state. The non-radiative transition of a singlet state to the triplet constitutes the phenomenon of intersystem crossing. ${ }^{228}$ Computationally predicting the chance of intersystem crossing during the electronic transitions that a molecule may undergo is possible through comparing the change in energy ( $\Delta \mathrm{E}_{\mathrm{abs}}$ ) during absorption (or emission) in both the singlet and triplet states. ${ }^{229}$ Hence, we performed calculations for the vertical excitations of all the molecules in their lowest energy triplet states. The methodology was kept the same as that for singlet state excitations. Table 5.9 shows the comparison of 
$\left.\Delta \mathrm{E}_{\text {abs }}\right)$ for both the states in all the four dyes.

In gas phase excitations, the maximum energy gap of $1.06 \mathrm{eV}$ is observed for $\mathbf{2}_{\mathrm{AB}}$ and $\mathbf{3}_{\mathbf{A B}}$. This is almost same in $\mathbf{4}_{\mathbf{A B}}$ with the value $1.04 \mathrm{eV}$. $\mathrm{S}_{1}$ and $\mathrm{T}_{1}$ in gas phase $\mathbf{1}_{\mathbf{A B}}$ are $0.97 \mathrm{eV}$ apart in energy terms. The energy difference of the two states in solvated molecules is even bigger. The largest difference is in case of $\mathbf{4}_{\mathrm{AB}}$ where it is $1.40 \mathrm{eV}$ while the smallest is for $\mathbf{3}_{\mathbf{A B}}(1.24 \mathrm{eV})$. For $\mathbf{1}_{\mathbf{A B}}$ and $\mathbf{2}_{\mathbf{A B}}$ it is $1.27 \mathrm{eV}$ and $1.36 \mathrm{eV}$ respectively.

Table 5.9: A comparison of the excitation energies $\left(\Delta \mathrm{E}_{\mathrm{abs}}\right)$ in $\mathrm{eV}$ for the first excited singlet and triplet states.

\begin{tabular}{c|cc|cc}
\hline & \multicolumn{2}{|c}{ Gas Phase } & \multicolumn{2}{c}{ Ethanol } \\
\hline & $\mathrm{S}_{1}$ & $\mathrm{~T}_{1}$ & $\mathrm{~S}_{1}$ & $\mathrm{~T}_{1}$ \\
\hline & $\Delta \mathrm{E}_{\mathrm{abs}}$ & $\Delta \mathrm{E}_{\mathrm{abs}}$ & $\Delta \mathrm{E}_{\mathrm{abs}}$ & $\Delta \mathrm{E}_{\mathrm{abs}}$ \\
\hline $\mathbf{1}_{\mathrm{AB}}$ & 2.77 & 1.80 & 2.39 & 1.12 \\
$\mathbf{2}_{\mathrm{AB}}$ & 2.88 & 1.82 & 2.59 & 1.23 \\
$\mathbf{3}_{\mathrm{AB}}$ & 2.88 & 1.82 & 2.55 & 1.31 \\
$\mathbf{4}_{\mathrm{AB}}$ & 2.85 & 1.81 & 2.55 & 1.15 \\
\hline
\end{tabular}

Although the $S_{1} \rightarrow T_{1}$ energy gaps in gas phase vertical calculations are significantly large, those in the calculations with solvation model are even larger which shows that the solvent tends to facilitate forbidding intersystem crossing, thus avoiding the nonradiative loss of energy during absorption or emission. This again supports the idea that the dyes under discussion are efficient in exhibiting fluorescence.

\subsection{Conclusion}

The current study is to investigate the photophysics involved in the fluorescence behaviour of selected 3-aminobenzanthrone dyes. We have optimised their ground state geometries to explore the structural features of these dyes. We further compared the changes happening upon their solvation in ethanol. It is evident from the values of dihedral angles that solvent causes changes in the geometries of the molecules. As expected from their structure with extended $\pi$ conjugation, the process of electronic 
transitions in these dyes is the result of intramolecular charge transfer which flows from the electron-donating $\mathrm{N}$-substituents on position 3 of the benzanthrone framework to the carbonyl group. However, this was only suspected previously and there was no evidence for that. For the first time we have presented unambiguous results to prove that, due to intramolecular charge transfer, these dyes can be potential candidates in manufacturing opto-electronic devices. The NTO analysis of vertical excitations shows how the charge density flows from the substituent through the benzanthrone framework to the carbonyl group. A polar solvent such as ethanol facilitates this whole process by inducing changes in the polarity. This is evident from enhanced dipole moments in solvated system calculations compared to the gas phase dyes at all the stages from ground state to vertical excitations to the excited state minima.

The mechanism of intramolecular charge transfer was further elaborated by means of NBO analysis including NBO charges and second order perturbation theory. It can be witnessed that upon solvation the individual charges on the atoms change (although to a small extent), which induce changes in polarity. Through FMO analysis, we were able to calculate the HOMO-LUMO band gaps by measuring the change in SCF energy. Here again, an increase in polarity decreases the band gap which facilitates the absorption and emission phenomena.

The computational results in the current study are in line with the experimental data published elsewhere. In vertical excitations, there is maximum of $12 \%$ error from the experimental results while in emission spectra it is maximally 5-6\%. However, these errors are in the range of the already reported error i.e. $23 \%$. The possibility of intersystem crossing was also estimated by comparing the vertical excitations of singlet state molecules with their corresponding triplet states. Very large energy differences suggest that this non-radiative loss is unlikely to occur. All these findings suggest that the benzanthrone dyes under discussion fluoresce efficiently. This study imparts significant insights from a future perspective. Detailed photophysics of the benzanthrone dyes under discussion is expected to inform the physical chemistry community about the un- 
derlying mechanism involved in the fluorescence of these molecules. The calculated charge accumulation and charge depletion regions over the benzanthrone framework, as a result of electronic excitations and the subsequent NTO analysis, will also pave the way for the synthetic perspective in search of new benzanthrone dyes by selecting appropriate charge-donating substituents. 


\section{Chapter 6}

\section{Summary and Outlook}

The study deals with the computational investigation of structural features and some other properties of selected donor-acceptor compounds. In the first part in addition to the geometry optimization, thermodynamic properties have been studied to compare the relative stability. of different coordination modes in metallic complexes with cyclophanes. The flexible structure of $p \mathrm{Cp}$ allows it to form more stable complexes with metals than comparatively more rigid Dp does. In fact, the former efficiently changes its configuration according to the metallic environment to attain higher stability while the latter can't. Moreover, reactivity of main-group metal heterobimetallic compounds has also been the subject of this study by investigating the nature of bonding.

To gain a deeper insight into the bonding interaction in $\eta^{18}$ inclusion complexes, EDA analysis was performed. There occurs a decrease in the overall interaction energy of Group 14 and $15 p$ Cp complexes compared to their Dp counterparts that can be attributed to the expected vibrational changes in $p \mathrm{Cp}$ complexes due to the flexible geometry. The calculation of interaction energy depends on two frozen fragments where vibrational changes are not taken into account.

The bonding properties of some recently known heterobimetallic compounds were investigated by carrying out the NBO and Bader's analyses. We showed that the In-Zn 
bond is of donor-acceptor nature which is the first ever known In-Zn bond. On the basis of Bader's analysis and, with the help of NBO charges, we suggested that indium in this bond behaves as a donor while zinc as acceptor.

In the final part, we computationally investigated the structure of selected benzanthrone dyes and the mechanism of fluorescence taking place in them. We compared the gas phase and solvation phase results which show that solvent plays a significant role in the fluorescence of dyes. These dyes were previously reported where ethanol solvent was used. Hence, we also selected ethanol solvent in the computational solvation model. These dyes possess extended $\pi$ conjugation on the basis of which it was always suspected that fluorescence shown by these dyes is due to the intramolecular charge transfer from the electron-donating substituents to the electron-accepting benzanthrone framework. It is now for the first time confirmed by computational chemistry that such a charge transfer actually takes place. The NTO analysis clearly shows the charge depletion from the donor parts and its accumulation on the acceptor sites.

An overall in-depth study was conducted involving vertical excitations and excited state optimization. The ethanolated excitations show a marked increase in polarity which means ethanol solvent assists in intramolecular charge transfer.

The study is expected to prove as a useful reference for synthetic and physical chemists. The first project i.e. the coordination and bonding properties of metal-cyclophane complexes can be helpful in search of new metal extractors to be used, for instance, in waste-water treatment. They might also find other enhanced industrial applications in future such as ion-selective electrodes based on the fact that $p \mathrm{Cp}$ has already proved to have this tendency toward $\mathrm{Ag}^{+}$. The selectivity and sensitivity of these $\pi$-prismands can be further altered by using imidazolium instead of benzene rings, introducing the coordinating substituents on the cyclophane cavity framework or by adjusting the size of the cavity to effectively host other metal cations. As mentioned previously, there is need to invest more efforts on the exploitation of such guest-host complexes. Moreover, this study can also assist the study of physical properties of metal-cyclophane 
donor-acceptor complexes, such as geometry and thermodynamic parameters.

The study involving the benzanthrone dyes provides an important insight into the photophysical properties of the selected luminophores. This shows that 3-aminobenzanthrone derivatives can be potential candidates in light harvesting materials such as liquid crystal display (LCD) devices and light emitting diodes (LEDs). Although there have been efforts in the past to synthesize and characterize these dyes, their application on industrial scale is yet to be explored. The need for opto-electronic devices is increasing day by day alongside the advancement in technology. These dyes with extended $\pi$ conjugation are excellent candidates in the field of optronics. Moreover, the previous findings show that 3-aminobenzanthrone dyes have proved efficient organic probes in membrane studies. However, the extended use in this regard can be computationally tested based on molecular modelling which has never been done to date to the best of our knowledge which is, however, not feasible with quantum mechanics. It can be concluded, based on the computational results presented here, particularly the NTO analysis, that the charge-donor substituents facilitate the charge transfer from the substituents to the benzanthrone framework. Moreover, by comparing the gas phase and ethanolated excited states, we propose that increasing polarity enhances intramolecular charge transfer that in turn induces a red-shifted emission in these dyes. 


\section{Bibliography}

1. Eremin, V. Chemical encyclopedia (in Russian). V. 2-M.: Sovetskaja ehnciklopedija; 2014; pp 463-464.

2. Emmett, L.; Prentice, G. M.; Pantoş, G. D. Annu. Rep. Prog. Chem., Sect. B: Org. Chem. 2013, 109, 217-234.

3. Frenking, G.; Tonner, R. The Chemical Bond; John Wiley \& Sons, Ltd, 2014; Chapter 4, pp 71-112.

4. Rivard, E. Dalton Trans. 2014, 43, 8577-8586.

5. Cárdenas-Jirón, G. I.; Zagal, J. H. J. Electroanal. Chem. 2001, 497, 55 - 60.

6. Reed, A. E.; Curtiss, L. A.; Weinhold, F. Chem. Rev. 1988, 88, 899-926.

7. Bent, H. A. Chem. Rev. 1968, 68, 587-648.

8. Kosower, E. M.; Dodiuk, H.; Tanizawa, K.; Ottolenghi, M.; Orbach, N. J. Am. Chem. Soc. 1975, 97, 2167-2178.

9. Kosower, E. M.; Kanety, H. J. Am. Chem. Soc. 1983, 105, 6236-6243.

10. Liptrot, D. J.; Power, P. P. Nat. Rev. Chem. 2017, 1, 0004.

11. Gui, J.; Marchon, B. Langmuir 1710, 5, 1999.

12. Desiraju, G. R.; Steiner, T. The Weak Hydrogen Bond: In Structural Chemistry and Biology; Oxford University Press, 2001; Vol. 9.

13. Dickson-Karn, N. M.; Olson, C. M.; Leu, W. C. W.; Hartley, C. S. J. Phys. Org. Chem. 2014, 27, 661-669.

14. Moore, G. E. Electronics 1965, 38, $114 \mathrm{ff}$.

15. Houk, K. N.; Cheong, P. H.-Y. Nature 2008, 455, 309-313.

16. Power, P. P. Nature 2010, 463, 171-177.

17. Frunzke, J.; Lein, M.; Frenking, G. Organometallics 2002, 21, 3351-3359.

18. Cossairt, B. M.; Cummins, C. C. J. Am. Chem. Soc. 2009, 131, 15501-15511.

19. Klinkhammer, K. W.; Fässler, T. F.; Grützmacher, H. Angew. Chem. Int. Ed. 1998, $37,124-126$. 
20. Stürmann, M.; Saak, W.; Marsmann, H.; Weidenbruch, M. Angew. Chem. Int. Ed. 1999, 38, 187-189.

21. Gynane, M. J. S.; Harris, D. H.; Lappert, M. F.; Power, P. P.; Riviere, P.; RiviereBaudet, M. J. Chem. Soc., Dalton Trans. 1977, 2004-2009.

22. Wu, J.; Yu, T.-L.; Chen, C.-T.; Lin, C.-C. Coord. Chem. Rev. 2006, 250, 602-626.

23. Wenkin, M.; Ruiz, P.; Delmon, B.; Devillers, M. J. Mol. Catal. A-Chem. 2002, $180,141-159$.

24. Kimura, H.; Tsuto, K.; Wakisaka, T.; Kazumi, Y.; Inaya, Y. Appl. Catal., A 1993, 96, 217-228.

25. Hanna, T. A. Coord. Chem. Rev. 2004, 248, 429-440.

26. Evans, P. A.; Cui, J.; Gharpure, S. J.; Hinkle, R. J. J. Am. Chem. Soc. 2003, 125, $11456-11457$.

27. Scheer, M.; Balázs, G.; Seitz, A. Chem. Rev. 2010, 110, 4236-4256.

28. Manners, I. J. Polym. Sci. Polym. Chem. 2002, 40, 179-191.

29. Takeuchi, A.; Inoue, A. Mater. Trans. 2005, 46, 2817-2829.

30. Weidenbruch, M. Eur. J. Inorg. Chem. 1999, 1999, 373-381.

31. Lee, V. Y.; Sekiguchi, A. Organometallics 2004, 23, 2822-2834.

32. Power, P. P. ChemComm 2003, 2091-2101.

33. Chen, Y.; Tan, C.; Zhang, H.; Wang, L. Chem. Soc. Rev. 2015, 44, 2681-2701.

34. Miller, M.; Quintana, J.; Ojeda, J.; Langan, S.; Thorp, S.; Pozzi, E.; Sztejnberg, M.; Estryk, G.; Nosal, R.; Saire, E.; Agrazar, H.; Graiño, F. Appl. Radiat. Isot. 2009, 67, S226-S229.

35. Hasenknopf, B. Front. Biosci. 2005, 10, 275.

36. Gouzerh, P.; Proust, A. Chem. Rev. 1998, 98, 77-112.

37. Watt, M. M.; Collins, M. S.; Johnson, D. W. Acc. Chem. Res 2012, 46, 955-966.

38. Pitt, M. A.; Johnson, D. W. Chem. Soc. Rev. 2007, 36, 1441-1453.

39. Garrison, J. C.; Simons, R. S.; Kofron, W. G.; Tessier, C. A.; Youngs, W. J. ChemComm 2001, 1780-1781.

40. Murakami, Y. In Cyclophanes II; Vögtle, F., Ed.; Springer Berlin Heidelberg: Berlin, Heidelberg, 1983; pp 107-155.

41. Yang, Y.; Ouyang, R.; Xu, L.; Guo, N.; Li, W.; Feng, K.; Ouyang, L.; Yang, Z.; Zhou, S.; Miao, Y. J. Coord. Chem. 2015, 68, 379-397. 
42. Kremer, A. B.; Andrews, R. J.; Milner, M. J.; Zhang, X. R.; Ebrahimi, T.; Patrick, B. O.; Diaconescu, P. L.; Mehrkhodavandi, P. Inorg. Chem. 2017, 56, 1375-1385.

43. Ghosh, S.; Gowda, R. R.; Jagan, R.; Chakraborty, D. Dalton Trans. 2015, 44, 10410-10422.

44. Atwood, D. A. Coord. Chem. Rev. 1997, 165, 267-296.

45. Haller, E. E.; Joos, B.; Falicov, L. M. Phys. Rev. B 1980, 21, 4729-4739.

46. Holligan, K.; Rogler, P.; Rehe, D.; Pamula, M.; Kornienko, A. Y.; Emge, T. J.; Krogh-Jespersen, K.; Brennan, J. G. Inorg. Chem. 2015, 54, 8896-8904.

47. Kumar, K.; Magerstadt, M.; Gansow, O. A. J. Chem. Soc., Chem. Comm. 1989, $145-146$.

48. Martin, T. M.; Wood, P. T.; Schimek, G. L.; Pennington, W. T.; Kolis, J. W. Inorg. Chem. 1995, 34, 4385-4391.

49. Hadjikakou, S. K.; Ozturk, I. I.; Banti, C. N.; Kourkoumelis, N.; Hadjiliadis, N. J. Inorg. Biochem. 2015, 153, 293-305.

50. Revunova, K.; Nikonov, G. I. Dalton Trans. 2015, 44, 840-866.

51. Hashiguchi, B. G.; Konnick, M. M.; Bischof, S. M.; Gustafson, S. J.; Devarajan, D.; Gunsalus, N.; Ess, D. H.; Periana, R. A. Science 2014, 343, 1232-1237.

52. Kuznetsov, A. E.; Boldyrev, A. I.; Li, X.; Wang, L.-S. J. Am. Chem. Soc. 2001, $123,8825-8831$.

53. Boldyrev, A. I.; Wang, L.-S. Chem. Rev. 2005, 105, 3716-3757.

54. Frenking, G.; Tonner, R.; Klein, S.; Takagi, N.; Shimizu, T.; Krapp, A.; Pandey, K. K.; Parameswaran, P. Chem. Soc. Rev. 2014, 43, 5106-5139.

55. Lee, V. Y.; Aoki, S.; Kawai, M.; Meguro, T.; Sekiguchi, A. J. Am. Chem. Soc. 2014, 136, 6243-6246.

56. Guo, J.-D.; Liptrot, D. J.; Nagase, S.; Power, P. P. Chem. Sci. 2015, 6, 6235-6244.

57. Calmano, W.; Hong, J.; Förstner, U. Water Sci. Tech. 1993, 28, 223-235.

58. Dykstra, C.; Frenking, G.; Kim, K.; Scuseria, G. Theory and Applications of Computational Chemistry: The First Forty Years; Elsevier, 2011.

59. Power, P. P. Chem. Rev. 1999, 99, 3463-3504.

60. Morales-Morales, D.; Jensen, C. G. M. The Chemistry of Pincer Compounds; Elsevier, 2011.

61. Fehlner, T. P. Inorganometallic Chemistry; Springer Science \& Business Media, 2013. 
62. Pierre, J. L.; Baret, P.; Chautemps, P.; Armand, M. J. Am. Chem. Soc. 1981, 103, 2986-2988.

63. Kang, H. C.; Hanson, A. W.; Eaton, B.; Boekelheide, V. J. Am. Chem. Soc. 1985, 107, 1979-1985.

64. Schmidbaur, H.; Bublak, W.; Huber, B.; Reber, G.; Müller, G. Angew. Chem. Int. Ed. Engl. 1986, 25, 1089-1090.

65. Schmidbaur, H.; Bublak, W.; Huber, B.; Müller, G. Organometallics 1986, 5, 1647-1651.

66. Scherer, O. J. Acc. Chem. Res. 1999, 32, 751-762.

67. Fleischmann, M.; Welsch, S.; Krauss, H.; Schmidt, M.; Bodensteiner, M.; Peresypkina, E. V.; Sierka, M.; Gröger, C.; Scheer, M. Chem. Eur. J. 2014, 20, 3759-3768.

68. Ahrens, L. H. Geochim. Cosmochim. Acta 1952, 2, 155-169.

69. Schorn, W.; Grosse-Hagenbrock, D.; Oelkers, B.; Sundermeyer, J. Dalton Trans. 2016, 45, 1201-1207.

70. Rush, R. M.; Yoe, J. H. Anal. Chem. 1954, 26, 1345-1347.

71. Colquhoun, H. M.; Henrick, K. J. Chem. Soc., Chem. Comm. 1981, 85-87.

72. Berry, D. E.; Hicks, R. G.; Gilroy, J. B. J. Chem. Educ. 2009, 86, 76.

73. Lappert, M. F.; Rowe, R. S. Coord. Chem. Rev. 1990, 100, 267-292.

74. Zheng, N.; Bu, X.; Vu, H.; Feng, P. Angew. Chem. Int. Ed. 2005, 44, 5299-5303.

75. Haddadpour, S.; Melullis, M.; Staesche, H.; Mariappan, C. R.; Roling, B.; Clérac, R.; Dehnen, S. Inorg. Chem. 2009, 48, 1689-1698.

76. Findeis, B.; Contel, M.; Gade, L. H.; Laguna, M.; Gimeno, M. C.; Scowen, I. J.; McPartlin, M. Inorg. Chem. 1997, 36, 2386-2390.

77. Schmeisser, D.; Schnell, R. D.; Bogen, A.; Himpsel, F. J.; Rieger, D.; Landgren, G.; Morar, J. F. Surf. Sci. 1986, 172, 455-465.

78. Schoeller, W. W.; Sundermann, A.; Reiher, M. Inorg. Chem. 1999, 38, 29-37.

79. Bauzá, A.; Mooibroek, T. J.; Frontera, A. Angew. Chem. Int. Ed. 2013, 52, 1231712321.

80. Weidenbruch, M. Organometallics 2003, 22, 4348-4360.

81. Power, P. P. Organometallics 2007, 26, 4362-4372.

82. Schwier, T.; Gevorgyan, V. Org. Lett. 2005, 7, 5191-5194.

83. Rit, A.; Campos, J.; Niu, H.; Aldridge, S. Nat. Chem. 2016, 8, 1022-1026. 
84. Stang, P. J. Chem. Rev. 1978, 78, 383-405.

85. Bruce, M. I. Chem. Rev. 1991, 91, 197-257.

86. Takagi, N.; Nagase, S. Organometallics 2001, 20, 5498-5500.

87. Stoumpos, C. C.; Malliakas, C. D.; Kanatzidis, M. G. Inorg. Chem. 2013, 52, 9019-9038.

88. Isobe, C.; Cho, H.-C.; Crowell, J. E. Surf. Sci. 1993, 295, 117-132.

89. Al-Rafia, S. M. I.; Malcolm, A. C.; Liew, S. K.; Ferguson, M. J.; Rivard, E. J. Am. Chem. Soc. 2011, 133, 777-779.

90. Thimer, K. C.; Al-Rafia, S. M. I.; Ferguson, M. J.; McDonald, R.; Rivard, E. ChemComm 2009, 7119-7121.

91. Groh, M.; Wolff, A.; Grasser, M.; Ruck, M. Int. J. Mol. Sci. 2016, 17, 1452.

92. Rogers, R. D.; Bond, A. H.; Aguinaga, S. J. Am. Chem. Soc. 1992, 114, 29602967.

93. Schwamm, R. J.; Lein, M.; Coles, M. P.; Fitchett, C. M. Angew. Chem. 2016, 128, 15018-15021.

94. Solyntjes, S.; Neumann, B.; Stammler, H.-G.; Ignat'ev, N.; Hoge, B. Chem. Eur. J. 2017, 23, 1568-1575.

95. Sadler, P. J.; Li, H.; Sun, H. Coord. Chem. Rev. 1999, 185-186, 689-709.

96. Swindell, E. P.; Hankins, P. L.; Chen, H.; Miodragović, D. U.; O’Halloran, T. V. Inorg. Chem. 2013, 52, 12292-12304.

97. Frenking, G.; Wichmann, K.; Fröhlich, N.; Loschen, C.; Lein, M.; Frunzke, J.; Rayón, V. M. Coord. Chem. Rev. 2003, 238-239, 55-82.

98. Kuczkowski, A.; Schulz, S.; Nieger, M.; Schreiner, P. R. Organometallics 2002, $21,1408-1419$.

99. Haaland, A. Angew. Chem. Int. Ed. Engl. 1989, 28, 992-1007.

100. Schulz, S.; Kuczkowski, A.; Nieger, M. J. Organomet. Chem. 2000, 604, 202207.

101. Baldwin, R. A.; Foos, E. E.; Wells, R. L.; White, P. S.; Rheingold, A. L.; Yap, G. P. A. Organometallics 1996, 15, 5035-5038.

102. Wells, R. L. Coord. Chem. Rev. 1992, 112, 273-291.

103. Wells, R. L.; Self, M. F.; McPhail, A. T.; Aubuchon, S. R.; Woudenberg, R. C.; Jasinski, J. P. Organometallics 1993, 12, 2832-2834.

104. Jones, L. J.; McPhail, A. T.; Wells, R. L. Organometallics 1994, 13, 3634-3638. 
105. Wells, R. L.; Baldwin, R. A.; White, P. S.; Pennington, W. T.; Rheingold, A. L.; Yap, G. P. A. Organometallics 1996, 15, 91-97.

106. Wells, R. L.; Purdy, A. P.; Higa, K. T.; McPhail, A. T.; Pitt, C. G. J. Organomet. Chem. 1987, 325, C7-C10.

107. Cowley, A. H.; Jones, R. A. Angew. Chem. Int. Ed. Engl. 1989, 28, 1208-1215.

108. Keehn, P. M.; Rosenfeld, S. M. Cyclophanes; Academic Press, New York, 1983; Vol. I and II.

109. Diedrich, F. N. Cyclophanes; Royal Society of Chemistry: Cambridge, 1991.

110. Probst, T.; Steigelmann, O.; Riede, J.; Schmidbaur, H. Chem. Ber. 1991, 124, 1089-1093.

111. Wong, Z. X.; Lein, M. Aust. J. Chem. 2016, 69, 969-974.

112. Gross, J.; Harder, G.; Siepen, A.; Harren, J.; Vögtle, F.; Stephan, H.; Gloe, K.; Ahlers, B.; Cammann, K.; Rissanen, K. Chem. Eur. J. 1996, 2, 1585-1595.

113. Cohen-Addad, C.; Baret, P.; Chautemps, P.; Pierre, J.-L. Acta. Crystallogr. C 1983, 39, 1346-1349.

114. Cohen-Addad, C.; Lebars, M.; Renault, A.; Baret, P. Acta Crystallogr. C 1984, 40, 1927-1931.

115. Ogoshi, H.; Sugimoto, H.; Yoshida, Z. Tetrahedron Lett. 1976, 17, 4481-4484.

116. Schmidbaur, H.; Hager, R.; Huber, B.; Müller, G. Angew. Chem. Int. Ed. Engl. 1987, 26, 338-340.

117. Probst, T.; Steigelmann, O.; Riede, J.; Schmidbaur, H. Angew. Chem. Int. Ed. Engl. 1990, 29, 1397-1398.

118. Kunze, A.; Gleiter, R.; Bethke, S.; Rominger, F. Organometallics 2006, 25, 47874791.

119. Ulloa, C. O.; Ponce-Vargas, M.; de Mattos Piccoli, R.; Caramori, G. F.; Frenking, G.; Munoz-Castro, A. RSC Adv. 2015, 5, 7803-7811.

120. Kutzelnigg, W. Angew. Chem. Int. Ed. Engl. 1984, 23, 272-295.

121. Lein, M.; Krapp, A.; Frenking, G. J. Am. Chem. Soc. 2005, 127, 6290-6299.

122. Phillips, A. D.; Wright, R. J.; Olmstead, M. M.; Power, P. P. J. Am. Chem. Soc. 2002, 124, 5930-5931.

123. Jambor, R.; Kašná, B.; Kirschner, K. N.; Schürmann, M.; Jurkschat, K. Angew. Chem. Int. Ed. 2008, 47, 1650-1653.

124. Davidson, P. J.; Lappert, M. F. J. Chem. Soc., Chem. Comm. 1973, 317.

125. Su, J.; Li, X.-W.; Crittendon, R. C.; Robinson, G. H. J. Am. Chem. Soc. 1997, 119, 5471-5472. 
126. Cordero, B.; Gomez, V.; Platero-Prats, A. E.; Reves, M.; Echeverria, J.; Cremades, E.; Barragan, F.; Alvarez, S. Dalton Trans. 2008, 2832-2838.

127. Grunenberg, J. Int. J. Quantum Chem. 2017, 117, e25359.

128. Molina, J. M.; Dobado, J. A.; Heard, G. L.; Bader, R. F. W.; Sundberg, M. R. Theor. Chem. Acc. 2001, 105, 365-373.

129. Tokitoh, N.; Arai, Y.; Okazaki, R. Phosphorus Sulfur Silicon Relat. Elem. 1997, 124, 371-378.

130. Pauling, L. J. Am. Chem. Soc. 1931, 53, 1367-1400.

131. Morokuma, K. J. Chem. Phys. 1971, 55, 1236-1244.

132. Dostál, L.; Jambor, R.; Růžička, A.; Jirásko, R.; Lyčka, A.; Beckmann, J.; Ketkov, S. Inorg. Chem. 2015, 54, 6010-6019.

133. Ghereg, D.; Ouhsaine, F.; Escudié, J.; Labat, S.; André, E.; Miqueu, K.; Sotiropoulos, J.-M.; Gornitzka, H.; Saffon, N. Organometallics 2013, 32, 10851093.

134. Kumar, R.; Mabrouk, H. E.; Tuck, D. G. J. Chem. Soc., Dalton Trans. 1988, $1045-1047$.

135. Beletskiy, E. V.; Wu, Y.; Kung, M. C.; Kung, H. H. Organometallics 2016, 35, 301-302.

136. Park, J.; Hong, S. Chem. Soc. Rev. 2012, 41, 6931-6943.

137. Butler, M. J.; Crimmin, M. R. ChemComm 2017, 53, 1348-1365.

138. Cammarota, R. C.; Lu, C. C. J. Am. Chem. Soc. 2015, 137, 12486-12489.

139. Lin, T.-P.; Ke, I.-S.; Gabbäi, F. P. Angew. Chem. Int. Ed. 2012, 51, 4985-4988.

140. Wade, C. R.; Gabbäi, F. P. Angew. Chem. Int. Ed. 2011, 50, 7369-7372.

141. Dange, D.; Choong, S. L.; Schenk, C.; Stasch, A.; Jones, C. Dalton Trans. 2012, 41, 9304-9315.

142. Krasovitskiň, B. M.; Bolotin, B. M. Organic Luminescent Materials; Wiley-VCH, 1988.

143. Bentley, P.; McKellar, J. F.; Phillips, G. O. J. Chem. Soc., J. Chem. Soc., Perkin Trans. 2 1974, 523-526.

144. Bentley, P.; McKellar, J. F.; Phillips, G. O. J. Chem. Soc., J. Chem. Soc., Perkin Trans. 2 1975, 1259-1262.

145. Bentley, P.; McKellar, J. F. J. Chem. Soc., Perkin Trans. 2 1976, 1850-1854.

146. Kirilova, E. M.; Belyakov, S. V.; Kirilov, G. K.; Kalnina, I.; Gerbreder, V. J. Lumin. 2009, 129, 1827-1830. 
147. Bulanovs, A.; Kirilov, G.; Fleisher, M.; Kirilova, E.; Mihailova, I. Opto-Electron. Rev. 2013, 21, 227-232.

148. Grabchev, I.; Bojinov, V.; Moneva, I. Dyes Pigm. 2001, 48, 143-150.

149. Gonta, S.; Utinans, M.; Kirilov, G.; Belyakov, S.; Ivanova, I.; Fleisher, M.; Savenkov, V.; Kirilova, E. Spectrochim. Acta A Mol. Biomol. Spectrosc. 2013, $101,325-334$.

150. Nepraš, M.; Machalický, O.; Šeps, M.; Hrdina, R.; Kapusta, P.; Fidler, V. Dyes Pigm. 1997, 35, 31-44.

151. Zhytniakivska, O.; Trusova, V.; Gorbenko, G.; Kirilova, E.; Kalnina, I.; Kirilov, G.; Kinnunen, P. J. Lumin. 2014, 146, 307-313.

152. Kirilova, E. M.; Kalnina, I.; Kirilov, G. K.; Meirovics, I. J. Fluoresc. 2008, 18, 645-648.

153. Khrolova, O. R.; Kunavin, N. I.; Komlev, I. V.; Tavrizova, M. A.; Trofimova, S. I.; Madii, V. A.; Petukhov, V. A. J. Appl. Spectrosc. 1984, 41, 771-775.

154. Wróbel, D.; Boguta, A.; Mykowska, E.; Bauman, D.; Grabchev, I. Mol. Cryst. Liq. Cryst. 2005, 427, 369-381.

155. Grabchev, I.; Moneva, I.; Wolarz, E.; Bauman, D. Dyes Pigm. 2003, 58, 1-6.

156. Bojinov, V. B.; Grabchev, I. K. Org. Lett. 2003, 5, 2185-2187.

157. Konstantinova, T. N. Dyes Pigm. 1989, 10, 63-67.

158. Staneva, D.; Vasileva-Tonkova, E.; Makki, M. S. I.; Sobahi, T. R.; AbdelRahman, R. M.; Asiri, A. M.; Grabchev, I. J. Photochem. Photobiol. B 2015, $143,44-51$.

159. Vus, K.; Trusova, V.; Gorbenko, G.; Sood, R.; Kirilova, E.; Kirilov, G.; Kalnina, I.; Kinnunen, P. J. Fluoresc. 2014, 24, 493-504.

160. Siddlingeshwar, B.; Hanagodimath, S. M.; Kirilova, E. M.; Kirilov, G. K. J. Quant. Spectrosc. Radiat. Transf. 2011, 112, 448-456.

161. Konstantinova, T. N.; Lazarova, R. A. Polym. Degrad. Stab. 2007, 92, 239-243.

162. Lu, B.; Xu, J.; Fan, C.; Miao, H.; Shen, L. J. Phys. Chem. B 2009, 113, 37-48.

163. Konstantinova, T.; Bojadgieva, J. Angew. Makromol. Chem. 1993, 205, 91-95.

164. Konstantinova, T.; Konstantinov, H.; Kaneva, T.; Spirieva, A. Polym. Degrad. Stab. 1998, 62, 323-326.

165. Ryzhova, O.; Vus, K.; Trusova, V.; Kirilova, E.; Kirilov, G.; Gorbenko, G.; Kinnunen, P. Methods Appl. Fluores. 2016, 4, 034007.

166. Kirilova, E. M.; Meirovics, I.; Belyakov, S. V. Chem. Heterocycl. Compd. 2002 , 38, 789-792. 
167. Debeaux, M.; Brandhorst, K.; Jones, P. G.; Hopf, H.; Grunenberg, J.; Kowalsky, W.; Johannes, H.-H. Beilstein J. Org. Chem. 2009, 5.

168. Umeda, R.; Namba, T.; Yoshimura, T.; Nakatsukasa, M.; Nishiyama, Y. Tetrahedron 2013, 69, 1526-1531.

169. Bradley, W. J. Chem. Soc. (Resumed) 1949, 2712-2715.

170. Kapusta, P.; Machalický, O.; Hrdina, R.; Nepraš, M.; Zimmt, M. B.; Fidler, V. J. Phys. Chem. A 2003, 107, 9740-9746.

171. Adamo, C.; Jacquemin, D. Chem. Soc. Rev. 2013, 42, 845-856.

172. Trusova, V. M.; Kirilova, E.; Kalnina, I.; Kirilov, G.; Zhytniakivska, O. A.; Fedorov, P. V.; Gorbenko, G. P. J. Fluoresc. 2012, 22, 953-959.

173. Brazdil, J. F.; Glaeser, L. C.; Grasselli, R. K. J. Catal. 1983, 81, 142-146.

174. Jensen, F. Introduction to Computational Chemistry; John Wiley and Sons Ltd., 2007.

175. Born, M.; Oppenheimer, R. Ann. Phys. 1927, 389, 457-484.

176. Slater, J. C. Phys. Rev. 1951, 81, 385-390.

177. Helgaker, T.; Jorgensen, P.; Olsen, J. Molecular Electronic-Structure Theory; John Wiley \& Sons, 2014.

178. Hohenberg, P.; Kohn, W. Phys. Rev. 1964, 136, 864-871.

179. Kohn, W.; Sham, L. J. Phys. Rev. 1965, 140, 1133-1138.

180. Parr, G., Robert; Yang, W. R. G. Density-Functional Theory of Atoms and Molecules; Oxford University Press, 1987.

181. Becke, A. D. Phys. Rev. A 1988, 38, 3098.

182. Lee, C.; Yang, W.; Parr, R. G. Phys. Rev. B 1988, 37, 785-789.

183. Perdew, J. P.; Yue, W. Phys. Rev. B 1986, 33, 8800-8802.

184. Perdew, J. P.; Chevary, J. A.; Vosko, S. H.; Jackson, K. A.; Pederson, M. R.; Singh, D. J.; Fiolhais, C. Phys. Rev. B 1992, 46, 6671-6687.

185. Perdew, J. P.; Burke, K.; Ernzerhof, M. Phys. Rev. Lett. 1996, 77, 3865-3868.

186. Adamo, C.; Barone, V. J. Chem. Phys. 1999, 110, 6158-6170.

187. Miehlich, B.; Savin, A.; Stoll, H.; Preuss, H. Chem. Phys. Lett. 1989, 157, 200206.

188. Grimme, S. J. Comput. Chem. 2004, 25, 1463-1473.

189. Grimme, S. J. Comput. Chem. 2006, 27, 1787-1799.

190. Grimme, S.; Antony, J.; Ehrlich, S.; Krieg, H. J. Chem. Phys. 2010, 132, 154104. 
191. Becke, A. D.; Johnson, E. R. J. Chem. Phys. 2005, 122, 154104.

192. Kang, R.; Chen, H.; Shaik, S.; Yao, J. J. Chem. Theory Comput. 2011, 7, 40024011.

193. Steinmetz, M.; Grimme, S. ChemistryOpen 2013, 2, 115-124.

194. Ditchfield, R.; Hehre, W. J.; Pople, J. A. J. Chem. Phys. 1971, 54, 724-728.

195. Schäfer, A.; Horn, H.; Ahlrichs, R. J. Chem. Phys 1992, 77, 8.

196. Schäfer, A.; Huber, C.; Ahlrichs, R. J. Chem. Phys. 1994, 100, 5829-5835.

197. Weigend, F. Phys. Chem. Chem. Phys. 2006, 8, 1057-1065.

198. Schlegel, H. B. Wiley Interdiscip. Rev. Comput. Mol. Sci. 2011, 1, 790-809.

199. Foresman, J.; Frish, E. Exploring Chemistry with Electronic Structure Methods, 3rd ed.; Gaussian Inc. USA, 2015.

200. Ziegler, T.; Rauk, A. Theor. Chim. Acta 1977, 46, 1-10.

201. Lein, M.; Szabo, A.; Kovacs, A.; Frenking, G. Faraday Discuss. 2003, 124, 365378.

202. Zhao, L.; von Hopffgarten, M.; Andrada, D. M.; Frenking, G. Wiley Interdiscip. Rev. Comput. Mol. Sci. 2018, 8, e1345.

203. Franck, J.; Dymond, E. G. Trans. Faraday Soc. 1926, 21, 536-542.

204. Condon, E. U. Phys. Rev. 1926, 27, 637-644.

205. Munakata, M.; Wu, L. P.; Ning, G. L. Coord. Chem. Rev. 2000, 198, $171-203$.

206. Galembeck, S. E.; Caramori, G. F.; Misturini, A.; Garcia, L. C.; Orenha, R. P. Organometallics 2017, 36, 3465-3470.

207. Baker, M. V.; Bosnich, M. J.; Williams, C. C.; Skelton, B. W.; White, A. H. Aust. J. Chem. 1999, 52, 823-826.

208. Bobacka, J.; Lahtinen, T.; Nordman, J.; Häggström, S.; Rissanen, K.; Lewenstam, A.; Ivaska, A. Electroynalysis (N.Y.N.Y.) 2001, 13, 723-726.

209. Schmidbaur, H. Angew. Chem. Int. Ed. Engl. 1985, 24, 893-904.

210. Schmidbaur, H.; Nowak, R.; Huber, B.; Müller, G. Organometallics 1987, 6, 2266-2267.

211. Schmidbaur, H.; Bublak, W.; Huber, B.; Müller, G. Angew. Chem. Int. Ed. Engl. 1987, 26, 234-236.

212. Schmidbaur, H.; Probst, T.; Steigelmann, O.; Müller, G. Heteroat. Chem. 1990, 1, 161-165.

213. Mantina, M.; Chamberlin, A. C.; Valero, R.; Cramer, C. J.; Truhlar, D. G. J. Phys. Chem. A 2009, 113, 5806-5812. 
214. Lide, D. R. CRC handbook of chemistry and physics; CRC press, 2004; Vol. 85.

215. Bondi, A. J. Phys. Chem. 1964, 68, 441-451.

216. Huber, K.-P. Molecular spectra and molecular structure: IV. Constants of diatomic molecules; Springer Science \& Business Media, 2013.

217. Anker, M. D.; Altaf, Y.; Lein, M.; Coles, M. P. Dalton Trans. 2019, page numbers not yet assigned, DOI:10.1039/C9DT03990B.

218. Popelier, P. L. A. The Chemical Bond; John Wiley \& Sons, Ltd, 2014; Chapter 8, pp 271-308.

219. Trusova, V. M.; Kirilova, E.; Kalnina, I.; Kirilov, G.; Zhytniakivska, O. A.; Fedorov, P. V.; Gorbenko, G. P. J. Fluoresc. 2012, 22, 953-959.

220. Krasovitskii, B. M.; Bolotin, B. Khimiya, Moscow 1984, 1230.

221. Grabchev, I.; Bojinov, V.; Moneva, I. Dyes Pigm. 2001, 48, 143 - 150.

222. Kirilova, E. M.; Kalnina, I.; Kirilov, G. K.; Meirovics, I. J. Fluoresc. 2008, 18, 645-648.

223. Nepraš, M.; Machalický, O.; S̈eps, M.; Hrdina, R.; Kapusta, P.; Fidler, V. Dyes Pigm. 1997, 35, 31 - 44.

224. Demchenko, A. P. Introduction to Fluorescence Sensing; Springer Science \& Business Media, 2008.

225. Shivraj,; Siddlingeshwar, B.; Kirilova, E. M.; Belyakov, S. V.; Divakar, D. D.; Alkheraif, A. A. Photochem. Photobiol. Sci. 2018, 17, 453-464.

226. Allen, F. H.; Kennard, O.; Watson, D. G.; Brammer, L.; Orpen, A. G.; Taylor, R. J. Chem. Soc., Perkin Trans. 2 1987, S1-S19.

227. Altaf, Y. Synthesis, Characterization and DFT Studies of New Benzanthrone Derivatives. M.Sc. thesis, Department of Chemistry, COMSATS Institute of Information Technology Islamabad (Abbottabad Campus), Pakistan, 2014.

228. McNaught, A. D.; Wilkinson, A.; Jenkins, A. D. Compendium of Chemical Terminology; Blackwell Science Oxford, 1997; Vol. 1669.

229. Beyhan, S. M.; Gotz, A. W.; Ariese, F.; Visscher, L.; Gooijer, C. J. Phys. Chem. A 2011, 115, 1493-1499.

230. Dunford, C. L.; Smith, G. J.; Swinny, E. E.; Markham, K. R. Photochem. Photobiol. Sci. 2003, 2, 611-615. 


\section{Appendix}

The SCF energy and xyz coordinates of the optimized structures and some selected figures for magnification purposes are provided in the $\mathrm{CD}$ attached. Alternatively, these can be found at:

https://github.com/yasiraltaf00/phd-thesis-suppl-info/ releases/tag/v2.3.4 